2. TO: (Receiving Organization) FSAR Project

5. Proj ./Prog./Dept./Div.:

FSAR Project/Safety \& Licensing/TRWS Engineering

8. Originator Remarks:

This document provides the results of the Tank Waste Remediation System Final Safety Analysis Report (TWRS FSAR) hazards identification/facility configuration activities undertaken from the period of March 7, 1996 to May 31, 1996, performed by personnel from WHC and SAIC.

11. Receiver Remarks:
3. From: (Originating organization)

Plant Systems Technical

Basis/SAIC

6. Cog. Engr.:

Danny Mendoza
4. Related EDT No.:

$N / A$

7. Purchase order No.:

$N / A$

9. Equip./Component No.:

$N / A$

10. System/Bldg./Facility:

Tank Farms

12. Major Assm. Dwg. No.:

$N / A$

13. Permit/Permit Application No.: N/A

14. Required Response Date:

N/A

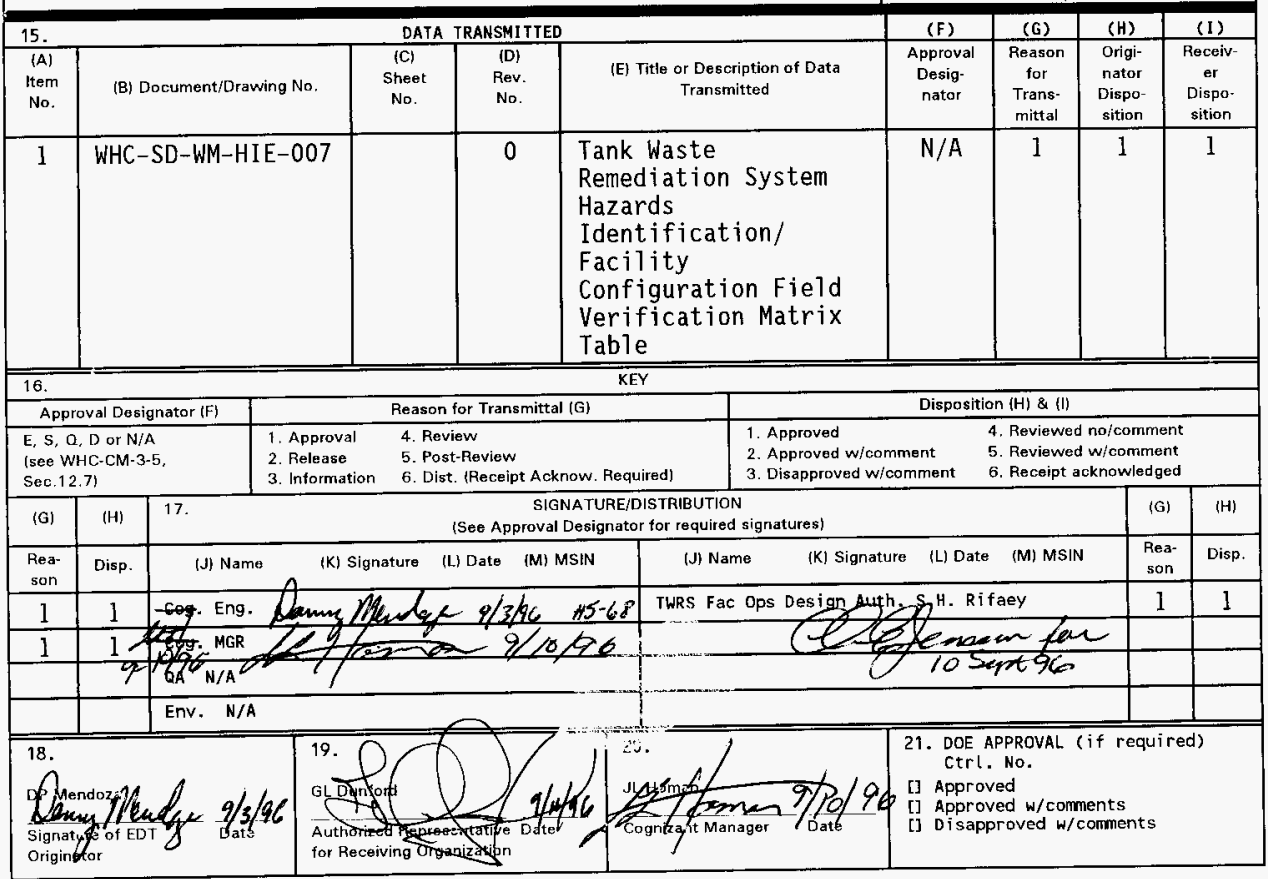




\title{
Tank Waste Remediation System FSAR Hazard Identification/Facility Configuration Verification Report
}

\author{
Danny P. Mendoza \\ Westinghouse Hanford Company, Richland, WA 99352 \\ U.S. Department of Energy Contract DE-AC06-87RL10930
}

\begin{tabular}{|c|c|c|}
\hline $\begin{array}{l}\text { EDT/ECN: } \\
\text { Org Code: } \\
\text { B\&R Code: }\end{array}$ & $\begin{array}{l}606479 \\
74 E 50 \\
\text { EW3120071 }\end{array}$ & $\begin{array}{l}\text { UC: } 2000 \\
\text { Charge Code: } \\
\text { Total Pages: }\end{array}$ \\
\hline
\end{tabular}

Key Words: Facility Configuration, Hazards Analysis, Hazards, Tank Farm Configuration, Hazards Identification

Abstract: This document provides the results of the Tank Waste Remediation System Final Safety Analysis Report (TWRS FSAR) hazards identification/facility configuration activities undertaken from the period of March 7, 1996 to May 31, 1996. The purpose of this activity was to provide an independent overview of the TWRS facility specific hazards and configurations that were used in support of the TWRS FSAR hazards and accident analysis development. It was based on a review of existing published documentation and field inspections. The objective of the verification effort was to provide a "snap shot" in time of the existing TWRS facility hazards and configurations and will be used to support hazards and accident analysis activities.

TRADEMARK DISCLAIMER. Reference herein to any specific commercial product, process, or service by trade name, trademark, manufacturer, or otherwise, does not necessarily constitute or imply its endorsement, recommendation, or favoring by the United States Government or any agency thereof or its contractors or subcontractors.

Printed in the United States of America. To obtain copies of this document, contact: WHC/BCS

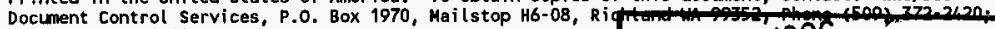
Fax (509) 376-4989.
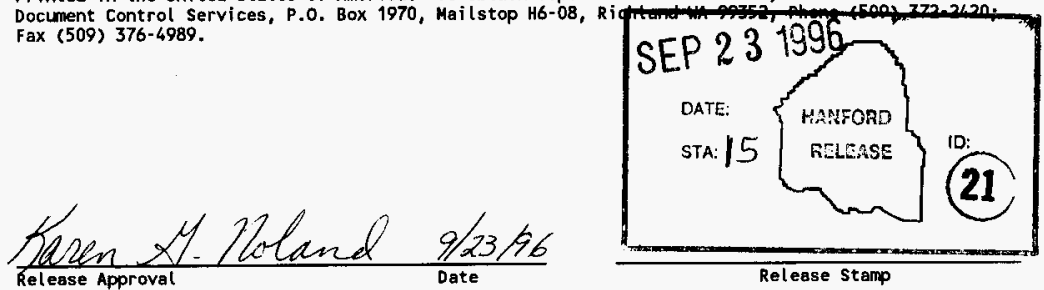

Rel ease Stamp 
Tank Waste Remedintion System FSAR Hazard Identification/Facility Configuration Verification Report

May 1996

\author{
Prepared for: \\ Westinghouse Hanford Company \\ Tank Waste Remediation System \\ FSAR Development \\ P.O. Box 1970 \\ Richland, Washington 99352
}

Prepared by:

Science Applications International Corporation 3250 Port of Benton Boulevard

Richland, Washington 99352

WHC Contract No. MW6-SWV-168681 
This page intentionally left blank. 


\section{EXECUTIVE SUMMARY}

This document provides the results of the Tank Waste Remediation System Final Safety Analysis Report (TWRS FSAR) hazard identification/facility configuration activities which were undertaken from the period of March 7 through May 31, 1996. The purpose of the hazands and configuration verification activity was to provide an independent overview of TWRS facility-specific hazards and configurations that were used in support of the TWRS FSAR hazards and accident analysis development. The scope of this effort addressed all current TWRS-owned facilities within the scope of the FSAR. It was based on a review of existing published documentation and field inspections. The objective of the verification effort was to provide a "snap shot" in time of the existing TWRS facilities hazards and configurations and will be used to support hazards and accident analysis activities.

This report contains an introduction section that provides for the background and associated purpose and scope of the activities; results of the verification activities that provides a summary of the data collection and review process; a discussion of the facility specific matrix data table development activities that included the identification of data needs and the generation of the actual data tables, training and pilot studies; and states the verification methodology that was employed during the document review and field verification activities.

The methodology employed for the verification activities included the development of facility specific matrix tables and associated activities which included:

- Development of an initial master checklist of TWRS facilities which bounds the project scope

- Division of the master checklist into twenty smaller checklists (one for each of the 18 tank farms, one for transfer lines, and one for miscellaneous facilities)

- Creation of six tables (checklists) of information to be compiled for all TWRS facilities (except transfer lines) through document review and field verification activities

- Creation of a seventh table specifically for transfer lines

- Preparation of instructions to assist in completion of the tables

- Training of personnel involved in the verification process

- Completion of a pilot study on AP Tank Farm.

- Incorporation of lessons learned from the AP Tank Farm Pilot

- Completion of checklists for the remainder of the TWRS facilities

In general, the verification activity results represent a "snap shot in time" of the TWRS facility configurations and potential hazards. Widely varying amounts and quality of data were available for the respective TWRS facilities. For example, double-shell tanks (DSTs) had generally up-to-date drawings, while single-shell tank (SST) drawings were, in general, not current with existing field conditions. Tank contents are well documented for the SSTs and DSTs. Less information was readily available for catch tanks, vault tanks, Inactive Miscellaneous Underground Storage Tanks (IMUSTs), and Double-Contained Receiver Tanks (DCRTs). The results obtained during the verification process will be utilized by the hazards/accident analysis group for use in the determination and evaluation of potential TWRS related hazards, facility configurations and accident scenarios associated with facility operations. 
Rev. 0

This page intentionally left blank. 
CONTENTS

EXECUTIVE SUMMARY

iii

1.0 INTRODUCTION $\ldots \ldots \ldots \ldots \ldots \ldots \ldots \ldots \ldots \ldots \ldots \ldots \ldots \ldots \ldots \ldots \ldots \ldots$

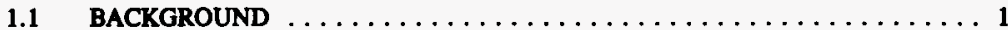

1.2 PURPOSE AND SCOPE $\ldots \ldots \ldots \ldots \ldots \ldots \ldots \ldots \ldots \ldots \ldots \ldots$

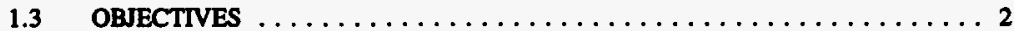

1.4 REPORT ORGANIZATION $\ldots \ldots \ldots \ldots \ldots \ldots \ldots \ldots \ldots \ldots \ldots \ldots \ldots$

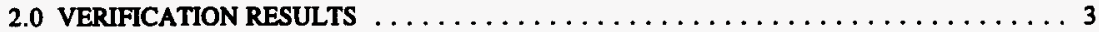

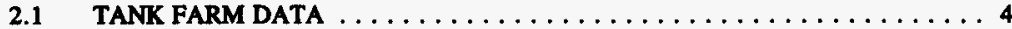

2.2 MISCELLANEOUS FACILITY DATA $\ldots \ldots \ldots \ldots \ldots \ldots \ldots \ldots$

2.3 TRANSFER LINE DATA $\ldots \ldots \ldots \ldots \ldots \ldots \ldots \ldots \ldots \ldots \ldots \ldots$

2.4 CORRECTIONS TO THE MASTER FACILITY CHECKLISTS $\ldots \ldots \ldots \ldots \ldots$. . 12

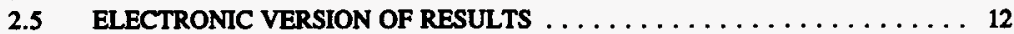

2.6 IDENTIFICATION/RESOLUTION OF OPEN ISSUES $\ldots \ldots \ldots \ldots \ldots \ldots \ldots$

3.0 MATRIX TABLE DEVELOPMENT $\ldots \ldots \ldots \ldots \ldots \ldots \ldots \ldots \ldots \ldots \ldots$

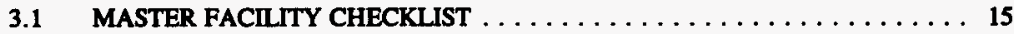

3.2 TABLE DEVELOPMENT $\ldots \ldots \ldots \ldots \ldots \ldots \ldots \ldots \ldots \ldots \ldots \ldots \ldots \ldots$

3.2.1 Table 1 - Materials Inventory $\ldots \ldots \ldots \ldots \ldots \ldots \ldots \ldots \ldots \ldots$

3.2.2 Table 2 - Hazards Identification $\ldots \ldots \ldots \ldots \ldots \ldots \ldots \ldots$

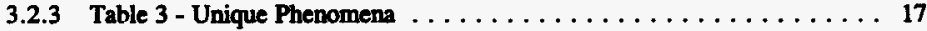

3.2.4 Table 4 - Facility Configuration $\ldots \ldots \ldots \ldots \ldots \ldots \ldots \ldots$

3.2.5 Table 5 - Radiation Source/Physical Protection . . . . . . . . . . 18

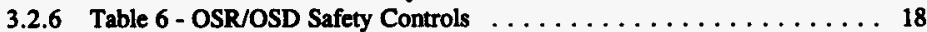

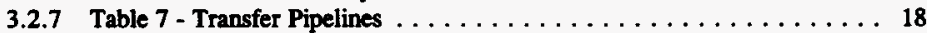

3.3 TABLE INSTRUCTIONS $\ldots \ldots \ldots \ldots \ldots \ldots \ldots \ldots \ldots \ldots \ldots \ldots$

3.4 TEAM DEVELOPMENT AND TRAINING $\ldots \ldots \ldots \ldots \ldots \ldots \ldots \ldots$

$3.5 \quad$ AP PILOT STUDY $\ldots \ldots \ldots \ldots \ldots \ldots \ldots \ldots \ldots \ldots \ldots \ldots \ldots$

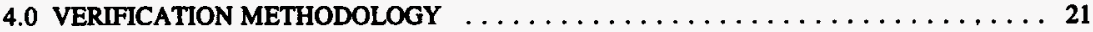

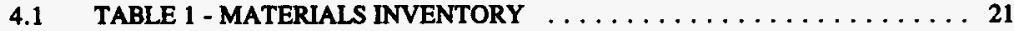

4.2 TABLE 2 - HAZARDS IDENTIFICATION $\ldots \ldots \ldots \ldots \ldots \ldots \ldots \ldots$

4.3 TABLE 3 - UNIQUE PHENOMENA .................. 22

4.4 TABLE 4 - FACILITY CONFIGURATION $\ldots \ldots \ldots \ldots \ldots \ldots \ldots \ldots$

4.5 TABLE 5 - RADIATION SOURCE/PHYSICAL PROTECTION . . . . . . . 23

4.6 TABLE 6 - OSD/OSR SAFETY CONTROLS $\ldots \ldots \ldots \ldots \ldots \ldots \ldots \ldots$

4.7 TABLE 7 - TRANSFER PIPELINES $\ldots \ldots \ldots \ldots \ldots \ldots \ldots \ldots \ldots$

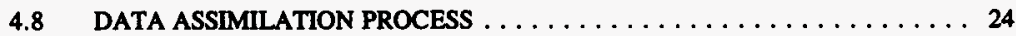

4.9 REVIEW/SIGN OFF PROCESS $\ldots \ldots \ldots \ldots \ldots \ldots \ldots \ldots \ldots \ldots \ldots$

5.0 SUMMARY AND CONCLUSIONS $\ldots \ldots \ldots \ldots \ldots \ldots \ldots \ldots \ldots \ldots \ldots \ldots$

6.0 REFERENCES . . . . . . . . . . . . . . . . . . . . . . . . . . 29 


\section{APPENDICES}
A MATRIX TABLE INSTRUCTION SHEETS
B 200 EAST AREA DOUBLE-SHELL TANK FARMS
C 200 WEST AREA DOUBLE-SHELL TANK FARMS
D 200 EAST AREA SINGLE-SHELL TANK FARMS
E 200 WEST AREA SINGLE-SHELL TANK FARMS
F MISCELLANEOUS FACILITIES
G TRANSFER LINES

\section{TABLES}

2-1 Summary of Results Organization Within the Appendices $\ldots \ldots \ldots \ldots \ldots \ldots$

2-2 Types of Facilities Found During Verification Activities Which Were Not on the

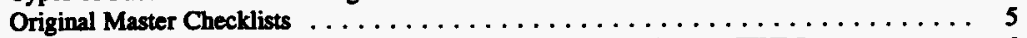

2-3 Facilities From Original Master Checklists Which Do Not Belong to TWRS ....... 6

2-4 Miscellaneous Facilities (Not Tied to a Tank Farm) $\ldots \ldots \ldots \ldots \ldots \ldots \ldots$. 9

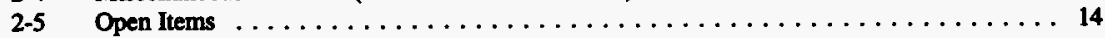


Rev. 0

This page intentionally left blank. 


\subsection{INTRODUCTION}

\subsection{BACKGROUND}

Westinghouse Hanford Company (WHC) is currently producing a Tank Waste Remediation System Final Safety Analysis Report (TWRS FSAR) document that will serve as the safety basis for TWRS operating facilities within the FSAR scope. An integral part of this consolidation process is the analysis of the hazards and identification of facility configurations associated with the operations of TWRS facilities.

The TWRS FSAR efforts required the review of TWRS documentation and interaction with TWRS personnel. The information gathered from this activity resulted in the generation of data to be incorporated in the FSAR. Due to the need to produce much of the FSAR in parallel, integration and verification of data for the hazard analysis and configuration identification was consolidated into one activity. This activity included a field walkdown effort to provide information on conditions (at the time of the walkdown) to support an assessment of the comprehensiveness and accuracy of the FSAR. The data gathered through these activities were captured in a table format for eventual conversion into a database structure to assist in the FSAR hazards/accident analysis activities and to support future activities.

\subsection{PURPOSE AND SCOPE}

The purpose of the TWRS FSAR hazard and configuration verification activity was to provide an independent verification of TWRS facility specific hazards and facility configurations which was used in support of the development of the TWRS FSAR. In some cases, multiple sources of information exist regarding hazards or configurations of TWRS facilities. The purpose of this document was to identify the appropriate analysis or information to be used in the FSAR. This was accomplished by document review, interviews, and field walkdown activities to identify and document TWRS facility specific hazards and configurations as they currently exist. This document reports the results of the verification process which occurred during the period between March 18, 1996 and May 31, 1996.

The scope of the effort included a review of all known TWRS owned facilities which will be covered in the FSAR document utilizing the existing published documentation and visual inspections. Hazards within TWRS, the configuration of TWRS systems and structures, and the existing operational controls were investigated. Field walkdowns were performed at the facility level (i.e., tank farm, building, or miscellaneous facility) and relied primarily on available published information and visual inspections of the accessible portions of the operating systems. No attempt was made during the field verification to review inaccessible portions of a given facility (e.g., contents within a valve pit or diversion box) as we employed a non-intrusive method of verification in accordance with existing WHC As Low As Reasonably Achievable (ALARA) principles.

The scope of the verification activities was to confirm the identification of:

1) Radioactive or hazardous materials in TWRS which potentially could pose a hazard to the public, onsite workers, TWRS workers, and/or the enviromment, 
2) Potential energy sources which could contribute to the uncontrolled release of material,

3) Unique phenomena associated with TWRS storage tanks which could contribute to the uncontrolled release of material,

4) TWRS facility systems, structures, and components (SSCs) related to the storage and transfer of waste,

5) Radiation protection features related to TWRS,

6) Operating Safety Requirements (OSRs) and Operating Specification Documents (OSDs) related to TWRS monitoring and control SSCs, and

7) Transfer lines related to the movement of liquid waste between facilities.

\subsection{OBJECTTVES}

The objective of the verification effort was to provide a "snap shot" in time of the existing TWRS facilities hazards and configurations. This facility and function specific information will be used to support the hazards and accident analysis, identification of radiation controls, and to confirm existing facility configurations. The data provided within the facility specific matrix tables support the hazard and accident analysis and the identification of necessary safety controls. The information contained in the report appendices may or may not be sufficient for other purposes.

\subsection{REPORT ORGANIZATION}

Section 1.0 outlines the background, purpose and scope, and organization of the report; Section 2.0 summarizes the results of the verification process; Section 3.0 explains the development of the matrix tables, including further definition of the project scope and objectives; Section 4.0 provides an overview of the verification process methodology; Section $\mathbf{5 . 0}$ provides a summary and conclusion of the verification activities; and Section 6.0 lists the references which were utilized. The appendices contain the instructions for completing the matrix tables and the actual matrix tables for each facility within the scope of the TWRS FSAR effort. 


\subsection{VERITCATION RESULTS}

The results of the verification activities are summarized in this section. The detailed data collected are provided in matrix tables and drawings located in Appendices B through G. Appendix A contains the instructions provided to assist in completion of the tables. The appendices have been organized by 200 East double-shell tanks (DSTs), 200 West DSTs, 200 East single-shell tanks (SSTs), 200 West SSTs, miscellaneous facilities, and transfer lines. Table 2-1 summarizes the organization of the Appendices.

Table 2-1. Summary of Results Organization Within the Appendices

\begin{tabular}{|c|c|c|}
\hline Appendix & TWRS Facilitie Included & Comments \\
\hline $\mathbf{A}$ & All* & $\begin{array}{l}\text { Instructions for completing motrix tebles are } \\
\text { provided in Appendix A. }\end{array}$ \\
\hline $\mathbf{B}$ & 200 East Area DSTs & $\mathbf{A N}, \mathbf{A P}, \mathbf{A W}, \mathbf{A Y}, \mathbf{A Z}$ Farms \\
\hline $\mathbf{c}$ & 200 West Area DSTs & SY Furm \\
\hline $\mathbf{D}$ & 200 East Area SSTs & $A, A X, B, B X, B Y, C$ Farm \\
\hline E & 200 Weat Ares SSTs & $\mathbf{S}, \mathbf{S X}, \mathbf{T}, \mathbf{T X}, \mathbf{T Y}, \mathbf{U}$ Farm \\
\hline $\mathbf{F}$ & Miscellaneous Fecilities & $\begin{array}{l}\text { See Table } 2-4 \text { for a listing of miscellaneous } \\
\text { fecilities. }\end{array}$ \\
\hline $\mathbf{G}$ & Transfer Lines & $\begin{array}{l}\text { Descriptive dan provided for active transfer lines } \\
\text { located between farms or facilities. Transfer lines } \\
\text { located within the boundaries of a tank fam and } \\
\text { insctive lines have been listed for ecountability, } \\
\text { with limited descriptive data provided. }\end{array}$ \\
\hline
\end{tabular}

In general, the results represent a "snap shot in time" of the TWRS facility configurations and potential hazards. Transient conditions are noted in the "Comment" columns wherever possible. Widely varying amounts and quality of data were available for the respective TWRS facilities. For example, DSTs had generally up-to-date, useful drawings, while SST drawings were, in general, not current with existing field conditions. Some information was available on the SST and DST tank contents (a few samples of DST supernate have been taken); less information was readily available for catch tanks, vault tanks, Inactive Miscellaneous Underground Storage Tanks (IMUSTs), and DoubleContained Receiver Tanks (DCRTs).

Matrix table results are organized by tank farm or miscellaneous facility. All "facilities" have been grouped by tank farm to the extent possible (Note: A "facility" could be a tank, building, crib, diversion box, etc.). Any facility with a tank farm letter in its identification (ID) number has been tied to that tank farm. For example, 241-BX-302B, which is an IMUST near B Plant, has been tied to BX Farm and is included with the BX Farm tables of information. This results in the majority of the TWRS owned facilities being tied to one of the tank farms and included with the information on that tank farm, even if the facility is physically located outside the boundaries of that farm. The remaining facilities, whose ID numbers did not include a tank farm designator, were deemed "Miscellaneous 
Facilities" and were reviewed and documented separately (see Appendix F). Transfer lines were also reviewed and documented separately (see Appendix G).

The matrix tables of data for each tank farm contained in Appendices B through $\mathbf{E}$ are briefly summarized in Section 2.1. The miscellaneous facility tables of data, found in Appendix F, are discussed in Section 2.2. Appendix $\mathbf{G}$, which contains data on transfer lines (Table 7), is summarized in Section 2.3.

Note: "NA" on the tables indicates the block was not applicable or not required for the stated purpose of the project.

\subsection{TANK FARM DATA}

For each tank farm, the Appendices contain a matrix table sign-off sheet which provides sign off of the data generator and data reviewer, a tank farm drawing which denotes the facility configuration observed during the field walkdown, a master checklist of "facilities" tied to the specific tank farm, and six matrix tables of information Material Inventory, Hazard Identification, Unique Phenomena, Facility Configuration, Radiation Source/Physical Protection, and OSR/OSD Safety Controls). The results are summarized for each table below:

Sirm-Ofi Sheet The signatures on the sign-off sheet signify that the information provided on this tank farm is based on the available reference documents, was field verified where possible, and is believed to be true, accurate, and complete at the time of review. Signatures of the team lead responsible for collating the information and the WHC cognizant engineer and design authority who reviewed the information are included.

Tank Farm Drawing Drawings were provided for each tank farm walkdown. The walkdowns were used to update and provide additional information (related to facility configuration and potential hazards) for the drawings. The drawings included in the Appendices reflect these field walkdown modifications. The locations and sizes of objects added to the drawings are approximations only.

Tonk Farm Master Facility Checllist Each walkdown team was provided with a master checklist of facilities for each tank farm (see Sections 3.0 and 4.0 for more information on the overall project methodology). The team lead was responsible for ensuring that all facilities were considered when filling out the matrix tables. An " $x$ " in the box indicates that that facility was included on that table.

All facilities which could not be accounted for are noted in the tank farm master checklists "Comment" columns and are discussed in Section 2.6. Additional facilities found during verification activities which were not on the original tank farm master checklists were added at the end of the checklists and are summarized in Table 2-2. Facilities from the master checklists which do not belong to TWRS are noted in the "Comments" column of the master checklists and are summarized in Table 2-3. 
Table 2-2. Types of Facilities Found During Verification Activities Which Were Not on the Original Master Checklists

\begin{tabular}{|l|l|}
\hline \multicolumn{1}{|c|}{ Fecilty Deacription } & \multicolumn{1}{|c|}{ Comments } \\
\hline Valve Pits & Found in A Farm, AX Furm, BY Farm, C Farm, and SX Farm \\
\hline Flush Pits & Found in SX Farm and U Farm \\
\hline Service Pits & Found in A Farm, BX Farm, C Farm, and U Farm \\
\hline Instrument Buildings & Found in BX Farm, BY Farm, SX Farm, T Farm, and U Farm \\
\hline Condenser Building & Found in SX Farm \\
\hline Compressor Buildings & Found in C Farm and SX Farm \\
\hline Sampling Buildings & Found in SY Farm \\
\hline Other Misc. Buildings (Water Building, & Found in A Farm \\
\hline Bentilation Building, Leat Detoction Lateral & \\
\hline Cribs & Found in A Farm, AZ Farm, and T Farm \\
\hline Caustic Addition Building and Skid & Found in AN Farm \\
\hline Surface Condenser Facility & Found in AX Farm \\
\hline Cross Connection Facility & Found in AX Farm \\
\hline DMUST & Found in TX Farm \\
\hline Diversion Boxes & Found in BX/BY Farm, T Farm, and TX Farm \\
\hline Clean Out Boxes & Found in S Farm and U Farm \\
\hline
\end{tabular}


Table 2-3. Facilities From Original Master Checklists Which Do Not Belong to TWRS

\begin{tabular}{|c|c|}
\hline Pacility & Current Omer \\
\hline $211 \mathrm{U}$ non-rad atorage tonk (Chemical Storage Tank) & BHI \\
\hline 211UA non-red suorage tank (Cold Chemical Make-up Tank) & BHI \\
\hline $276 U$ non-rad storage tank (Chemical Storage Tank) & BEI \\
\hline $211 T$ noa-rad torage thak (Chanical Storege Tank) & T Plant \\
\hline $\begin{array}{l}\text { 2706T201 non-rad storage tunk (Low Level Waste Decon } \\
\text { Tank) }\end{array}$ & T Pint \\
\hline $\begin{array}{l}2706 T 202 \text { non-red storage tank (Low Level Waste Decon } \\
\text { Tank) }\end{array}$ & T Flant \\
\hline 2115 nor-rad storage tmk (Chemical Storage Tank) & BHI \\
\hline 241-C-801 Process Stornge Building & Bechtel, monitored by TWRS \\
\hline 211B non-red storage tank (Chemical Storage Tank) & B Plane \\
\hline $\begin{array}{l}\operatorname{COB}-A w-01,-02,-03,-04,-05,-06,-07,-08,-11,-12, \\
\operatorname{COB}-A-30\end{array}$ & 242-A Evaporator \\
\hline 211A non-rad storage tank (Chemical Storage Tank) & Purex \\
\hline $\begin{array}{l}\text { 270A non-red storage tank (Underground Condensate } \\
\text { Neutralization Tank) }\end{array}$ & Purex \\
\hline 213-W-TK-1 IMUST & $\begin{array}{l}\text { Not owned by TWRS (WHC-SD-WM-PD-046, Rev. 0), but } \\
\text { included with } 213-\mathrm{W} \text { Compector tables under Miscellaneous } \\
\text { Facillities because it was noted during the 213-W walkdown. }\end{array}$ \\
\hline $216-8-3-3 *$ & BH \\
\hline 222EC non-rad storage tank (Emergency Generator) & 222-S Lab \\
\hline 284WA non-rad storage tank (Salt Brine Storage Tank) & 200-W Power House (Salt Brine Storage Tank) \\
\hline TR31-ST-T1 non-red storage tank (Leachate Storage Tank) & Solid Waste Disposal \\
\hline TR34-ST-T1 non-rad storage tank (Leachate Storage Tank) & Solid Waste Disposal \\
\hline 2711 E66 non-rad storage tank (Waste Oil Tank) & 200-E Garage \\
\hline 2711E66A non-rad storage tank (Waste Oil Tank) & 200-E Garage \\
\hline 2721E66 non-rad storage tank (Diesel Storage Tank) & 200-E Security Building \\
\hline Gasoline / Fuel Oil Storage Facility & Hanford Site (none specifically owned by TWRS. \\
\hline
\end{tabular}

- This we called 216-3-3 on the original master list. A.K. McDowell of WHC, Tank Farm Projects Group, provided this ID number. 


\section{Thble 1. Meteriel Inventorg}

The radioactive wastes stored in the DST and SSTs were the largest inventories of material found. No activity level measurements were found for pits or boxes. A minimal amount of analytical data on the curie content of failed High-Efficienty Particulate Air (HEPA) filters for use in accident analyses were found. In general, underground diesel fuel storage tanks, flammable storage cabinets, and contaminated equipment storage areas were identified in the field. Approximately $80 \%$ of the information on these tables was found in documents and could not be field verified, with the remainder being observed in the field.

\section{Table 2. Horands Identification}

Numerous hazards or potential hazards were identified including flammable materials (diesel fuel, hydrogen, sodium), corrosives (acids, caustics), electrical (wiring, motors, heaters), pressure/volume (gas bottles, surge tanks) and mass/gravity/height (stairs, cranes, ladders, pits). The categories of hazands were provided in a checklist from the FSAR hazard analysts.

\section{Table 3. Unique Fhencment}

Unique phenomena were only considered for single-shell, double-shell, and double-contained receiver tanks, per the FSAR Hazard/Accident analysts. Minimal documented data were available for the DCRTs. Minimal information was available on the potential for siphoning; however, a separate siphoning analysis is being performed by the FSAR hazards analysis group in parallel with this project. Hazards identified included high heat, flammable gas, ferrocyanide, corrosivity, and organics.

\section{Table 4. Feclity Configuration}

The configuration of all "facilities" found in each tank farm, including tanks, pits, boxes, buildings, instrumentation, and ventilation systems were identified. Not all tanks within a given farm have the same equipment (different pits, different ventilation methods, and different types of instrumentation exist on tanks within a given farm).

Instrumentation identified included waste level measurement, high level probes, leak detection instrumentation, temperature probes, and Continuous Air Monitors (CAMs). Some instrumentation was believed to be operable on certain tanks and inoperable on other tanks, while other instrumentation exists which does not work on any of the tanks within a given farm.

Monitoring systems were also found which do not work as originally designed, but the data were still being obtained (e.g., manual data collection was used where automatic data collection was designed). Instrumentation on diversion boxes, tanks, and pits which are isolated was generally not retrieved from old drawings.

Operability for some components was not easily obtainable. Operability of these components may be marked as "assumed operable" or "unknown" depending on the information available.

For double shell tanks and some DCRTs, condition assessment surveys and Project 314 reports were available and equipment condition scores (on a 1-10 scale) are reported from those 
sources. No sources of equipment condition scores were found for single shell tanks. No attempts to score the condition of equipment were made, so "NA" is reported wherever documented equipment scores were unavailable. Important information which did not conform to the columns of Table 4 is included in the "Comments" column. Differences among the tanks within a given farm have been noted by exception in the comment field.

The organization of Table 4 may not be readily apparent due to limited planning time. The organization of this table was designed to be general in nature to accommodate either DST or SST facilities. Tank specific information is generally at the beginning of the tables; valve pit, diversion box, DCRT, and building information is generally toward the end of the tables.

\section{Table 5, Radiation Sonrce / Phrsical Protection}

No surface readings of greater than $100 \mathrm{mR} / \mathrm{hr}$ were found on survey maps located at the respective facilities. All pits and tanks which could potentially have held radioactive materials were assumed to be greater than $100 \mathrm{mR} / \mathrm{hr}$, in the absence of any underground dose rate information. Cover blocks and penetrations were generally in good condition. The numbers and sizes of the penetrations and the cover block thicknesses are noted on the tables.

\section{Tohle 6, OSD/OSR Sofety Controns}

Several instances of inoperable equipment tied to OSR/OSD requirements were identified. The list of procedures associated with a specific OSR limit (provided in WHC-SD-WM-RPT-220) or OSD limit was tied to all tank farms; however, some of the procedures listed do not apply to all of the tank farms. Where comments have been received, corrections have been made. Numerous administratively controlled requirements exist which are not controlled or monitored by the SSCs identified on Tables 4 and 6 . While these OSD/OSR requirements are of interest to cognizant engineers, design authorities, and others, they are not presently included in the scope of Table 6.

\subsection{MISCELLANEOUS FACILTY DATA}

Appendix F contains data on the miscellaneous facilities owned by TWRS. Miscellaneous facilities discussed in Appendix $F$ are listed in Table 2-4.

The data collected on miscellaneous facilities are similar to the data collected for each tank farm, described in Section 2.1. For each of the miscellaneous facilities identified in Table 2-4, the appendix contains a sign-off sheet and five tables of information. Table 3 on Unique Phenomena was not required for the miscellaneous facilities, per TWRS FSAR hazards/accident analysts. All the IMUSTs not associated with individual tank farms are grouped together and all the cribs, ponds, and ditches not associated with individual tank farms are grouped together for simplicity.

The information provided in Appendix $F$ is summarized below.

Miscellanenus Facility Master Checklist. The first table in Appendix F is the original miscellaneous facility master checklist. This was the checklist of facilities which had not been tied to the tank farms during the planning stage. Note that many miscellaneous inactive 
Table 2-4. Miscellaneous Facilities (Not Tied to a Tank Farm)

\begin{tabular}{|c|c|}
\hline Feclity Number & Deocrtption \\
\hline 204-AR & Unloading Facility \\
\hline 209-E & 90 Day Storage Ped \\
\hline 213-W & Compector Building \\
\hline 241-AR-151 & Diversion Box \\
\hline 241-ER-151 & Divervion Box \\
\hline 241-ER-152 & Diversion Box \\
\hline 241-ER-153 & Diversion Box \\
\hline 241-ER-311 & Cutch Tank \\
\hline 241-EW-151 & Croes Site Vent Sution \\
\hline 244-AR & AR Vault \\
\hline $2727-W$ & Sodium Stornge Burilding \\
\hline 2727-WA & Storare Building \\
\hline 216-W-LC & Crib \\
\hline $216-2-20$ & Crib \\
\hline $216-2-21$ & Pond \\
\hline 243-S-TK-1 & DMUST \\
\hline 241-ER-311A & IMUST \\
\hline 231-W-151-001 & IMUST \\
\hline $231-W-151-002$ & MUST \\
\hline 241-Z-8 & MMUST \\
\hline
\end{tabular}


storage facilities (MISFs) were located during tank farm walkdowns and are covered with the individual tank farm data. An attempt was made to capture all MISFs, but no checklist was available to ensure that all MISFs were captured.

The team lead was responsible for ensuring that all facilities on the checklist were considered when filling out Tables 1 through 6 . An " $x$ " in the box indicates that that facility was included on that table. Some facilities do not belong to TWRS. These facilities are noted in the miscellaneous facility master checklist "Comment" column and were included in Table 2-3. Facilities which could not be accounted for (remain as open issues) are also noted in the miscellaneous facility master checklist "Comment" column and discussed in Section 2.6.

Sion-Off Shest The signatures on this sheet signify that the information provided for the miscellaneous facility is believed to be true, accurate, and complete. Signatures of the team lead and the reviewing Westinghouse cognizant engineer and design authority are included, where applicable. Not all miscellaneous facilities had assigned cognizant engineers ("NA" is noted on the respective miscellaneous facility sign-off sheets).

\section{Thble 1. Material Inventory}

Tank content data for the IMUSTs were limited and dated (WHC-EP-0861, WHC-SD-EN-ES040 , and the Environmental Sites Database were the sources of information recommended by WHC). Mixed and hazardous waste material inventories were found at the 209-E 90 Day Storage Pad. Historical information on material discharged to cribs, ponds, and ditches was taken from the Environmental Sites Database. However, additional data for the cribs, ponds, and ditches (including some sample results) may be available in the Liquid Effluent Monitoring Information System (LEMIS). The LEMIS database was not investigated during this project. Other materials, such as underground diesel fuel storage tanks, chemical cleaners, and other non-radioactive chemicals were found at various miscellaneous facilities during walkdowns.

\section{Table 2. Hazards Identification}

Hazards varied greatly from facility to facility. Hazards included corrosives, electrical, flammable materials, and kinetic energy sources. No thermal radiation or chemical reaction hazards were identified at miscellaneous facilities. The categories of hazards were provided in a checklist from the FSAR hazard analysts.

\section{Table 3. Unique Phengmeng}

Table 3 was not required for miscellaneous facilities, per TWRS FSAR Hazards and Accident Analysis representatives.

\section{Table 4. Facility Confiauration}

The configuration of the miscellaneous facility, including any tanks, pits, boxes, buildings, instrumentation, and/or ventilation systems was identified on Table 4. Instrumentation included waste level measurement, high level probes, leak detection instrumentation, temperature probes, and CAMs. Operability for some components was not easily obtainable. Operability of these components may be marked as "assumed operable" or "unknown" depending on the 
information available. No sources of equipment condition scores were found for the miscellaneous facilities.

None of the IMUSTS, cribs, ponds, or ditches (discussed in this section) have any operable instrumentation due to the fact that they are all inactive. (Several IMUSTs which were tied to individual tank farms in this report do have operable monitoring instrumentation). The 244-AR vault is currently in standby mode and has ventilation systems, instrumentation, and other equipment which has not been used for years and is currently assumed to be inoperable. Detailed information on the configuration of all miscellaneous facilities can be found on Table 4 for each miscellaneous facility in Appendix F.

Tohle 5, Radiation Soarce / Physical Protection

No surface readings of greater than $100 \mathrm{mR} / \mathrm{hr}$ were found on survey maps located at the respective facilities. Any pit or tank which could potentially hold radioactive materials was assumed to be greater than $100 \mathrm{mR} / \mathrm{hr}$. Diversion box cover blocks and penetrations were generally in good condition. The mumbers and sizes of the penetrations are noted on the tables from walkdowns; cover block thickness taken from drawings are also noted. For the AR vault, 244-AR, and the unloading facility, 204-AR, shielding wall thickness were also obtained from referenced drawings.

\section{Table 6. OSD/OSR Snfty Controls}

Numerous OSDs (related to the ventilation systems) exist for the 244-AR vault which are not currently being met since the facility is in standby mode. No OSR/OSD requirements were found for the SSCs associated with the IMUSTs, cribs, ponds, or ditches, 2727-W, 2727-WA, 209-E, 213-W, or the diversion boxes covered in this section. Numerous administratively controlled requirements exist which are not controlled or monitored by the SSCs identified on Tables 4 and 6. While these OSD/OSR requirements are of interest to cognizant engineers, design authorities, and others, they are not currently included in the scope of Table 6.

\subsection{TRANSFER LINE DATA}

Active transfer lines (except those contained entirely within the bounds of a single tank farm) are described on Table 7. The following descriptive data are provided for each of the active interfarm transfer lines: line ID, diameter, line type (concrete encased, pipe-in-pipe, etc.), material of construction, capacity, design pressure, connection facilities, transfer frequency, hydrotesting frequency, leak detection methods, presence of heat tracing, presence of backflow/siphoning prevention, ventilation systems, presence of cathodic protection, and associated diversion boxes.

Extensive descriptive data are not provided for transfer lines within the boundaries of a single tank farm, drain lines to catch tanks, cribs, ponds, and ditches, or steam lines, as these lines are not key to any of the TWRS FSAR accident scenarios. These lines are identified in Table 7 for FSAR Chapter 2 accountability/inventory purposes only.

The only OSR/OSD requirements associated with transfer lines are WHC-SD-WM-SAR-016, 11.7 and OSD-T-151-00007, 7.2.10 which deal with verifying operability of leak detectors on transfer lines prior to transfers. 


\subsection{CORRECTIONS TO THE MASTER FACILTTY CHECKLISTS}

One of the significant results of the verification activity was that the original checklists of facilities owned by TWRS were incomplete and occasionally incorrect. Through document reviews, interviews, and tank farm field walkdowns, additional facilities were found which are believed to belong to TWRS. Even if these facilities were currently inactive, they were added to the end of the tank farm specific master checklists (provided in the Appendices) and were summarized in Table 2-2.

Document review and interviews also indicated that TWRS is not responsible for some of the facilities on the original checklists. Specific items have been noted on the respective farm matrix tables. These facilities were summarized in Table 2-3.

\subsection{ELECTRONIC VERSION OF RESULTS}

All data have been provided in an electronic format in addition to the matrix table hard copies. Tables 1 through 5 are provided in Word Perfect 5.1/5.2 format. Table 6 is provided in an Access 2.0 database. A Crystal Report Writer $\mathbf{4 . 0}$ file is inchuded to produce printouts of the Access database. Table 7 is provided in Excel 5.0. All diskettes contents are clearly labeled.

\subsection{DENTTFICATION/RESOLUTION OF OPEN ISSUES}

Several open issues remain after the document and field verification activities described above. These items are discussed below and summarized in Table 2-5:

- The small set of systems or components which will form the safety basis for TWRS will require a more thorough validation of operability and complete documentation of the procedures associated with their use, calibration, maintenance, etc. This is beyond the scope of this effort and will be managed separately by WHC.

- Table 6 lists procedures which may not apply to the specific tank farm. The procedures listed were referenced in WHC-SD-WM-RPT-220 and the OSD Database (Kahlia 1996). These documents were the basis of reference for procedures which are associated with a given type of facility (e.g., DSTs, SSTs, DCRTs), not a specific tank farm.

- Table 6 also does not provide the complete accountability of all the OSDs and OSRs, which design authorities and cognizant engineers indicated during review. In the current Table 6 data, OSR/OSDs have been linked to a given SSC identified for each facility. If the OSR/OSD could not be linked to an SSC and was determined to be administratively controlled, it was considered not currently within the scope of Table 6 and was not identified.

- The HEPA filter radiation exposure rate limit is specified as $1 \mathrm{R} / \mathrm{hr}$ in WHC-CM-7-5, Rev. 1, but SD-WM-SAR-016, Rev. 1 states that administrative procedures exist to maintain HEPA filters below $200 \mathrm{mR} / \mathrm{hr}$. It is unclear which limit applies to which HEPA filters. Clarification by WHC is required to resolve this issue. 
- No analytical sample data were located for cribs, ponds, and ditches. Toward the end of the project, the LEMIS (Liquid Effluent Monitoring Information System) database was recommended as a potential source of sample data for the cribs, ponds, and ditches, but was not reviewed due to schectule constraints. This database should be reviewed for potentially useful material inventory data on cribs, ponds, and ditches.

- A number of facilities initially identified by FSAR activities were not found during the walkdowns and/or could not be found in documents or drawings. 
Table 2-5. Open Items

\begin{tabular}{|c|c|c|}
\hline Ope I Ine / Paclitiy & Status & Revolution \\
\hline HEPA Fiher exposure raxe limit & $\begin{array}{l}\text { Two references locited (WHC-CM-7-5, } \\
\text { Rev. } 1 \text { and SD-WM-SAR-016, Rev. 1); } \\
\text { unvure of their apolicability }\end{array}$ & $\begin{array}{l}\text { WHC policy decision / clarification } \\
\text { required. }\end{array}$ \\
\hline $\begin{array}{l}\text { Crib, pond, and ditch material } \\
\text { inventory }\end{array}$ & $\begin{array}{l}\text { Environmental Sites Datebase } \\
\text { informetion provided, but LEMIS may } \\
\text { have more useful informetion }\end{array}$ & $\begin{array}{l}\text { LEMIS should be reviewed for potentially } \\
\text { useful sample data on cribs, ponds, and } \\
\text { ditches. }\end{array}$ \\
\hline Table 6 objectives & $\begin{array}{l}\text { Table } 6 \text { provides applicable OSR/OSD } \\
\text { monitored/controlled by SSCs on Table } 4 \\
\text { only. Administrative controls are not } \\
\text { included in Table } 4 \text { scope. }\end{array}$ & $\begin{array}{l}\text { Revision of Tabie } 6 \text { to reflect all } \\
\text { OSR/OSDs and implementing procectures } \\
\text { not currently in project scope. Additional } \\
\text { WHC input and datsbese restructuring } \\
\text { required to support this ectivity if deemed } \\
\text { necessary by WHC. }\end{array}$ \\
\hline $\begin{array}{l}\text { Clean out bores COB-AP-07D, } \\
\text { COB-AP-OTE, COB-AP-OTF }\end{array}$ & $\begin{array}{l}\text { Not found in AP Farm or in drawings. } \\
\text { Believed to have been part of Grout } \\
\text { Program, but never built. }\end{array}$ & Verification by WHC required. \\
\hline $\begin{array}{l}\text { Airlift circulator } 601 \text { caissons in } \\
\text { AY Farm }\end{array}$ & $\begin{array}{l}\text { Not found on drawings or in the field. } \\
\text { Believed to have been planned, but never } \\
\text { installed. }\end{array}$ & Verification by WHC required. \\
\hline 216-B-63 Ditch & $\begin{array}{l}\text { Documentation found on this ditch, but it } \\
\text { could not be found in the field. }\end{array}$ & Verify existence in field. \\
\hline 215A non-rad storage tonk & $\begin{array}{l}\text { No information found in documentation } \\
\text { or drawings; not found in the field. }\end{array}$ & Verification by WHC required. \\
\hline $240-C-154$ diversion box & $\begin{array}{l}\text { Not found during walkdowns. Routing } \\
\text { board shows a } 241-C-154 \text { diversion box } \\
\text { which is isolated. This diversion box is } \\
\text { believed to be buried next to Hot Sem } \\
\text { Works, } 201-\text { C, across the street and west } \\
\text { of C Farm (WHC-SD-EN-ES-019) }\end{array}$ & Issue closed. Diversion box is buried. \\
\hline 2415303 non rad storage tank & $\begin{array}{l}\text { Drawings exist for this tank, but it is } \\
\text { believed to have never been built. }\end{array}$ & Verification by WHC required. \\
\hline 240-T-154 diversion box & $\begin{array}{l}\text { No drawings found; not found during } \\
\text { field walkdowns. }\end{array}$ & Verification by WHC required. \\
\hline $\begin{array}{l}\text { 241HN701-66 non-rad storage } \\
\text { tank }\end{array}$ & $\begin{array}{l}\text { Diesel storage tank for new tank farm. } \\
\text { Believed never built. }\end{array}$ & Verification by WHC required. \\
\hline 242A211 non-rad storage tank & $\begin{array}{l}\text { No information; not found in field. May } \\
\text { have been planned for } 242-\mathrm{A} \text {, but not } \\
\text { built. }\end{array}$ & Verification by WHC required. \\
\hline 241-AZ-562 Valve pit & $\begin{array}{l}\text { No information found in documentation } \\
\text { or drawings; not found in the field. }\end{array}$ & Verification by WHC required. \\
\hline 241-A-302A Catch tank & $\begin{array}{l}\text { Documentation found, but not seen } \\
\text { during walkdown (located near PUREX) }\end{array}$ & Field verification required. \\
\hline 241-A-151 Diversion box & $\begin{array}{l}\text { Documentation found, but not seen } \\
\text { during walkdown (behind Purex) }\end{array}$ & $\begin{array}{l}\text { Obtain access to rear, fenced area behind } \\
\text { Purex and field verify diversion box }\end{array}$ \\
\hline
\end{tabular}




\subsection{MATRIX TABLE DEVELOPMENT}

During the development process for the facility specific matrix tables, several activities occurred which defined the scope of the project. These activities included:

- Development of an initial master checklist of TWRS facilities which bounds the project scope

- Division of the master checklist into twenty smaller checklists (one for each tank farm, one for transfer lines, and one for miscellaneous facilities)

- Creation of six tables (checklists) of information to be compiled for all TWRS facilities (except transfer lines) through document review and field verification activities

- Creation of a seventh table specifically for transfer lines

- Preparation of instructions to assist in completion of the tables

- Training of personnel involved in the verification process

- Completion of a pilot study on AP Tank Farm.

- Incorporation of lessons learned from the AP Tank Farm Pilot

These activities which occurred during the planning phase are discussed below.

\subsection{MASTER FACLITY CBECKLIST}

A master checklist of facilities owned by TWRS was created during the planning phase. This checklist was intended to bound the facilities to be covered under the verification project. Draft versions of the FSAR Chapter 2, "Facilities Description and Operation", and the FSAR Chapter 3, "Hazards Analysis Topography" were used to create the initial master checklist of facilities. Transfer lines found in WHC-SD-W314-ES-021, Rev. 0 were added to the transfer lines identified in the draft of FSAR Chapter 2.

The master checklist was then subdivided into smaller master checklists for verification support as follows:

- $\quad$ one for each of the 18 tank farms

- $\quad$ one for transfer lines

- $\quad$ one for the remaining miscellaneous facilities, such as 204-AR, 209-E, etc.

During the planning phase, any facility with an ID number which included a tank farm designator was added to the tank farm master checklist for accountability purposes. For example, facilities with a "B" in the ID number was assigned to the B Farm master checklist. This resulted in 
some of the IMUSTs, cribs, ponds, and ditches being assigned to tank farms because of their ID number, even though they are not physically located within the tank farm. For example, the expansion ponds (216-B-3), were included with B Farm. IMUSTs, cribs, ponds, and ditches which could not be tied to a tank farm through their ID number were included on the miscellaneous facilities checklist.

During the course of the verification project, additional facilities were added to the original checklists, generally after field walkdowns. In some instances, facilities on the original checklists were identified as no longer owned by TWRS. These findings are noted in the "Comment" column of the individual master checklists (as found in the Appendices) and have been summarized in Section 2.4.

\subsection{TABLE DEVELOPMENT}

To define the project scope in terms of the data required, authors contributing to the FSAR were interviewed to determine which of their information needs could be supported by the verification project. The Facilities Description and Operation, Hazands and Accident Analysis, and Radiation Protection Program chapters of the FSAR were ultimately determined to be the primary drivers for the verification project.

Six tables were initially created. It was requested that the tables be robust enough to be used for all tank farms. Generally all of the six tables were filled out for each tank farm or miscellaneous facility. A seventh table was added to collect data related to TWRS transfer lines which was filled out once for all pertinent transfer lines.

The scope of each of the seven tables is discussed below.

\subsubsection{Table 1 - Materials Inventory}

A definition of the materials at risk for release was needed for accident analyses. Twelve categories of materials at risk were identified by the accident analysis representatives:

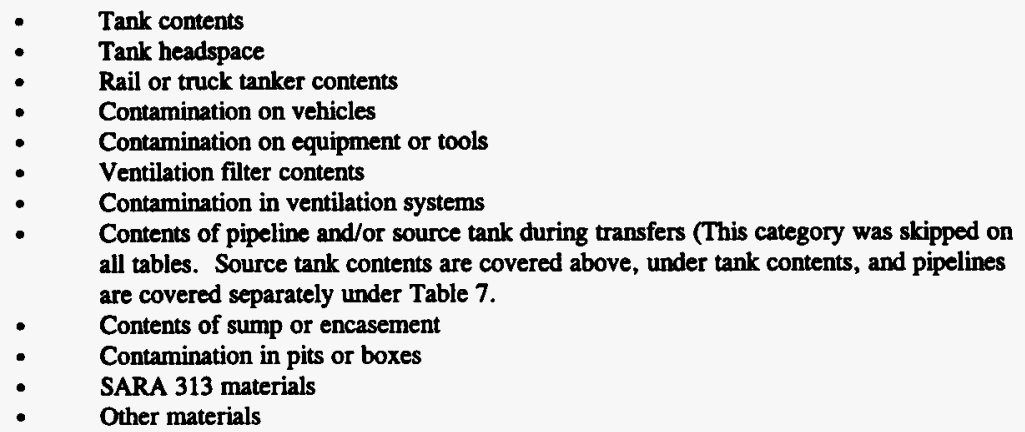

For each category of material at risk, Accident Analysis required the capacity of the container, a description of the material at risk (including the physical form, volume or activity, and whether the 
material was transient), and the quantity of containers (for example, the number of drums or bottles, if applicable). Table 1, Materials Inventory, was designed to fulfill these data requirements.

\subsubsection{Table 2 - Hazards Identification}

Hazard Identification required verification of the types of hazards present. For each "facility" identified on the master list and for each additional facility identified during field walkdowns, currentlyexisting hazards were to be identified. An existing checklist of potential hazard designators was provided by the FSAR hazard analysts and utilized for hazard verification. This checklist of hazards and the master checklist of facilities were used to create Table 2, Hazards Identification.

\subsubsection{Table 3 - Unique Phenomena}

Hazard/accident analysis and identification of controls required identification of any unique phenomena associated with waste storage tanks. All double-shell, single-shell, and double-contained receiver tanks were to be evahuated against the following potentially unique phenomena:

- Flammable gas

- Ferrocyanide

- Organics

- Separable phase

- $\quad$ TOC $>10 \mathrm{~g} / \mathrm{L}$ in liquids

- $\quad$ TOC $>3$ wt\% in solids on a dry basis)

- High heat (the definition of high heat as a unique phenomena varied from tank farm to tank farm based on the definitions provided in the OSDs. High heat criteria ranged from $>40,000 \mathrm{BTU} / \mathrm{hr}$ to $>70,000 \mathrm{BTU} / \mathrm{hr}$.)

- Pyrophoric materials

- Siphoning

- Criticality ( $>1 \mathrm{~g} / \mathrm{L}$ in solids)

- Corrosivity (corrosive material exceeding OSD limits)

These informational requirements led to the development of Table 3, Unique Phenomena. Any documented discounted events (i.e., studies which resolved specific tank related issues) were also considered when filling out this table and were noted where applicable.

\subsubsection{Table 4 - Facility Configuration}

Identification of what "facilities" currently exist and appear operable was the main type of information required to support the FSAR facility description (Chapter 2) and identification of safety controls. A "facility" was loosely defined to include structures such as buildings, tanks, pits, boxes, cribs, ponds, and ditches. For each facility which existed and appeared operable, existing substructures (such as a pit above a tank), ventilation, instrumentation, and other subsystems were to be identified and their operability assessed. Table 4, Facility Configuration, was designed to fulfill these informational requirements. 


\subsubsection{Table 5 - Radiation Source/Physical Protection}

For each of the facilities identified on Table 4 which could be thought of as a "location", the Radiation Protection Program section of the FSAR required an assessment of whether the location represented a radiation source greater than $100 \mathrm{mR} / \mathrm{hr}$. Tanks, pits, boxes, etc., were all considered "locations"; instrumentation was not considered a location. For all facilities thought to be greater than $100 \mathrm{mR} / \mathrm{hr}$, the authors of the Radiation Protection Program also needed a listing and description of the protective features employed. Specific descriptive information requested included the mumber and size of any penetrations, thickness of protective features such as cover blocks, and the condition of the cover blocks, cover plates, and penetrations. These informational requirements led to the development of Table 5, Radiation Source / Physical Protection.

\subsubsection{Table 6 - OSR/OSD Safety Controls}

Once completed, the FSAR will identify a set of systems, structures, and components (SSCs) which will be relied upon to provide mitigation or prevention of various potential accidents. To support this need of the FSAR, existing OSR and OSD requirements and implementing procedures which are known to be monitored or controlled by the systems, structures, and components identified on Table 4 were to be identified. Table 6, OSD/OSR Safety Controls, which contains all the SSCs from Table 4, was created to collect the currently existing requirements which are monitored or controlled by these SSCs and any procedures associated with these OSR/OSD requirements.

\subsubsection{Table 7 - Transfer Pipelines}

During the planning phase of the project, it became clear that transfer lines would be best handled on a separate checklist. The authors of the Facility Description and Accident Analysis sections of the FSAR were consulted and a list of questions for this checklist was developed in a brainstorming session. Since only active transfer lines between farms or facilities are considered in FSAR accident scenarios, a joint decision was made to focus the detailed transfer line study on active transfer lines. The scope was further defined by omitting the short nuns of transfer piping which are fully contained within a tank farm. Lines that were outside this scope were listed for FSAR Chapter 2 accountability purposes.

\subsection{TABLE INSTRUCTIONS}

Instructions were prepared for each of the above tables to assist in the uniform completion of each table. These instructions were designed to ensure relative consistency in data collection and data recording and have been provided in Appendix $\mathbf{A}$.

\subsection{TEAM DEVELOPMENT AND TRAINING}

Three teams were established to perform the document and field verification work. Each team consisted of a team lead, document review support personnel, and facility specific support personnel. The facility specific support personnel consisted of a cognizant engineer, a design authority, and/or an operations representative familiar with the particular tank farm or miscellaneous facility under review. 
Each team included a member responsible for hazard identification and a member responsible for facility configuration. Employees of Science Applications International Corporation (SAIC), Stone \& Webster, Westinghouse Hanford Company, and Kaiser Engineering Hanford participated on the three teams.

Two main training sessions were held, one prior to the AP Pilot Study on March 22, 1996 and one after the AP Pilot Study on March 28, 1996 (prior to kick-off of the main verification effort). These training sessions were intended to orient the participants on the objectives of, and methodology employed in, the TWRS FSAR Verification Project. A lessons-learned meeting was also held immediately following the AP Pilot Study on March 25, 1996 to incorporate issues that were encountered during the AP Pilot Study.

\subsection{AP PILOT STUDY}

Once draft versions of Tables 1 through 6 were prepared, a pilot study was completed on AP Tank Farm. Document reviews were expected to be completed prior to initiation of field walkdowns, but due to schectule constraints, the field walkdown for AP Tank Farm was completed prior to extensive document review. The pilot study revealed the importance of performing as much document review as possible prior to the field walkdown in order to make best use of the time in the field. The pilot study demonstrated to the verification team the type of information which might be visible in a brief facility walkdown. The cognizant engineer and operations personnel familiar with the facility were found to be crucial to the verification process, since they had knowledge of their systems which would be difficult, if not impossible, to locate in documents. They were also able to point the verification team members to the most useful and up-to-date documents and drawings on their facilities. The pilot study also allowed the potential pitfalls associated with gaining entry to the tank farms to be resolved.

Following the AP Pilot Study, tables populated with the data obtained on AP Tank Farm were sent out for review to the ultimate users of the information (i.e., the FSAR authors). Their comments and the comments of the cognizant engineer involved with the Pilot Study were used to finalize the tables. 
Rev. 0

This page intentionally left blank. 


\subsection{VERIFICATION METHODOLOGY}

Due to the quantity of documentation available for review, the document review process was completed by numerous personnel from SAIC, Stone and Webster, ICF Kaiser Hanford, and Westinghouse Hanford Company. The original project plan called for document reviews to be completed prior to initiation of the field walkdown. In reality, time constraints sometimes forced field walkdowns to occur without thorough document review. In these cases, information from documents was backfilled after the field walledowns were completed.

An initial list of the types of documents to be used in the document review was prepared during the planning phase of the project. Drawings, databases, supporting documents (SDs), external publications, procedures, roundsheets, Condition Assessment Reports, Project 314 Reports, and OSR/OSDs were included on the original list. The list was contimually expanded throughout the course of the project, as new information became available. A complete list of document references used in the process is provided in Section 6.0. The individual tables also include all references used; references are either listed next to a given data field or at the end of the table.

Field verification was performed through facility walkdowns. Team members, including a cognizant engineer, design authority, and operations personnel where applicable, viewed accessible portions of the tank farm facilities. No attempt was made to remove cover blocks or cover plates or to access restricted, contaminated areas such as the 244-AR Vault canyon. This was not intended to be a walkdown for configuration management as-builting of all of TWRS, but rather to identify the current configuration of TWRS facilities and currently visible hazards. The information as presented in the matrix tables represents a "snap-shot in time" view of TWRS facilities.

Access to some portions of TWRS for field verification was limited by construction activities, fresh air zones, or special training requirements. An attempt was made to locate qualified personnel to inspect all possible locations; areas not reviewed on walkdowns are noted on the individual master checklists.

For each of the seven tables, the types of documents which were reviewed and the ways in which field verification was used to support completion of the tables are summarized below.

\subsection{TABLE 1 - MATERIALS INVENTORY}

Tank contents were obtained from WHC-EP-0182-93 for SSTs and DCRTs and from the Tusler report 740A30-96-008 dated 2/27/96 for DSTs. IMUST contents were taken from WHC-EP-0861 and WHC-SD-EN-ES-040.

Tank vapor space volumes were obtained from the "TWINS" database. Headspace radionuclide concentrations were obtained from WHC-SD-WM-SARR-037. Ammonia amounts in the headspace were obtained from either the TWINS database, report PNL-10189 "Toxicologic Evaluation of Analytes from Tank 241-C-103", or from information obtained from WHC Industrial Hygiene Programs (personal interviews with Kyleen Smith and Elton Hewitt). For the WHC Industrial Hygiene Program information, all vapor samples came from areas likely to be indicative of concentrations in the double- and single-shell tanks. Typical sample areas were tank risers, breather filters, exhauster filters, and pump pits. The data cover a period from January 1993 to February 1996. 
Only maximum ppm values were used. This resulted in most data coming from 1995 and 1996. Data have been corrected for temperature. Since the pressure did not vary a great deal, it was assumed to be a constant 29.2 inches of mercury (about $14.3 \mathrm{psi}$ ). Actual pressures varied from 28.65 to 29.78 inches of mercury. Temperatures varied from 0 to $84^{\circ} \mathrm{C}$. Elton Hewitt of WHC Industrial Hygiene indicated that the accuracy of the ammonia measurements may deviate $\pm 25 \%$.

Analytical data on the curie content of failed HEPA filters was obtained for a small number of HEPA filters from WHC-SD-WM-EMP-031, Rev. 2. The capacity (maximum activity levels) for HEPA filters was identified as $200 \mathrm{mR} / \mathrm{hr}$ for DSTs in WHC-WM-SAR-016. However, WHC-CM-7-5 states that HEPA filters will be replaced when continuous exposure rates exceed $1 \mathrm{R} / \mathrm{hr}$ at six inches. The $1 \mathrm{R} / \mathrm{hr}$ number was reported for non-DST (e.g., DCRT, SST) HEPA filters.

Drawings and various documents were utilized to find capacities of pits, sumps, and tanks. SARA 313 materials were taken from the Hazardous Material Inventory Database, Inventory Verification by Reporting Representative Report dated 1/10/96.

The Environmental Sites Database was used to find data on the types of materials historically discharged to cribs, ponds, and ditches. Another database, the Liquid Effluent Monitoring Information System (LEMIS), was identified as another potential source of information on crib, pond, and ditch material inventory, but due to time constraints was not investigated further.

Field verification of materials inventory consisted of identifying visible materials within buildings or within the tank farms. Tanks, bottles, and drums of chemicals, gas bottles, and containers of contaminated material were observed in the field. Interviews with cognizant engineers, design authorities, and operations personnel were also used to verify information on material inventory when no other sources of information were available. Refer to the references provided on the individual tables for the exact references utilized.

\subsection{TABLE 2 - HAZARDS IDENTIFICATION}

The OSDs for SSTs and DSTs were utilized as a reference which indicated the presence of caustics, nitrates, and gases within the tanks. OSD-T-151-00030, Rev. B-17 was used to identify which tanks were watchlist or recommended watchlist tanks. Drawings which indicated various pieces of equipment which could be potential hazards were also utilized.

The field walkdown was utilized to verify visible hazards, such as flammable materials, friction sources, electrical equipment, kinetic-rotation and kinetic-linear hazards, mass-gravity-height hazards, and pressure volume hazards. Interviews with cognizant engineers, design authorities, and operations personnel were also used to verify the presence of hazards, particularly where visual confirmation was not possible.

\subsection{TABLE 3 - UNIQUE PHENOMENA}

Unique phenomena were characterized for the DSTs, SSTs, and DCRTs only. Table 3 information was acquired entirely through document review or interviews with cognizant personnel by the WHC Process Engineering Group. In some instances, the source reports used to develop the recommendations for watchlist tank designations were used as sources of information. 
No field verification of unique phenomena was attempted, due to the nature of the materials (i.e., the tank contents) involved.

\subsection{TABLE 4 - FACILITY CONFIGURATION}

Multiple sources of documentation were utilized to populate Table 4. Drawings, riser studies (SD-RE-TI-053 and SD-RE-TI-093), Interim Safety Basis (WHC-SD-WM-ISB-001), SARs for miscellaneous facilities such as 204-AR and 244-AR, roundsheets, and procedures were all used to identify what systems, structures, and components were present in a given farm or miscellaneous facility. Roundsheets were used in some instences to verify the operability of instrumentation. Equipment condition scores were all taken from documents, either Condition Assessment Surveys, or Project 314 Reports (WHC-SD-W314-ES-018, WHC-SD-W314-ES-020, and WHC-SD-W314-ES021). Equipment condition scores ranged from 1 to 10 , with 10 being the best condition. No documented sources of equipment condition scores for single shell tanks was found during the document review process.

Field verification was used to verify the existence of all visible systems, structures, and components. The operability of some equipment could be observed or assumed through field walkdowns, as well. Generally, the condition of equipment was not reported based on field walkdowns, unless obvious damage of a given item was observed. Shift office routing boards were used to verify the isolation status of some tanks, pits, and diversion boxes. Interviews with cognizant engineers, design authorities, and operations personnel were used as required to verify the existence or operability of systems, structures, or components not visible on field walkdowns. As noted above, no attempts were made to remove cover blocks to view internal contents of pits or boxes.

\subsection{TABLE 5 - RADIATION SOURCE/PHYSICAL PROTECTION}

Limited sources of documentation were found to indicate whether locations were greater than $100 \mathrm{mR} / \mathrm{hr}$. For surface locations, radiation survey maps were reviewed, with no locations greater than $100 \mathrm{mR} / \mathrm{hr}$ identified. The East Tank Farms High/Very High Radiation Area Log, dated 5/2/96 was obtained, which identified several locations which are monitored weekly, but all were indicated as below $100 \mathrm{mR} / \mathrm{hr}$ on the 5/2/96 survey. Requests for similar information from West Tank Farms received no response. For below surface contamination, all pits, radioactive material storage tanks, and diversion boxes were assumed to be greater than $100 \mathrm{mR} / \mathrm{hr}$.

Protective feature descriptions (e.g., cover block thicknesses) were obtained from drawings. Soil thicknesses above SSTs and DSTs were obtained from the corresponding OSDs.

Field walkdowns were used to verify the condition of protective features and to document the estimated the size and number of cover block penetrations, which will be used during the analysis of a spray accident evident.

\subsection{TABLE 6 - OSD/OSR SAFETY CONTROLS}

Table 6 was created from an existing database on OSRs (reference WHC-SD-WM-RPT-220, Rev. 0 ) and a database created in parallel with this project on OSDs (OSD Requirements Database, 
Kahlia, 1996). The OSDs utilized were currem as of 4/17/96. The purpose of Table 6 is to relate existing OSR/OSDs and associated implementing procedures to specific SSCs identified on Table 4 for each specific facility. Any requirements which were not tied to systems, structures, or components (SSCs) in the above databases were not tied to SSCs in this table. Those requirements which are not monitored or controlled by an SSCs have not been included in Table 6 .

This relation of safety requirement to SSC will allow the hazard and accident analysts to determine if appropriate controls are currently in place for the specific safety controls determined through their analysis to be required. The purpose of Table 6 for the verification project is not to provide a comprehensive listing of all OSR/OSDs and implementing procedures at this time.

The verification column included in Table 6 (FV $=$ field verified, $D R=$ document review) represents the information available in WHC-SD-WM-RPT-220 only. No additional field or document verification was performed to support Table 6 as part of this project.

\subsection{TABLE 7 - TRANSFER PIPELINES}

Since the transfer lines are below ground, all data were taken from source documentation. Numerous supporting documents, external publications, and the Operations Transfer Pipeline Routing Board were sources of information as indicated in the references listed at the end of Table 7.

The field verification activities were limited to inspection of the diversion boxes to which the pipelines connect (i.e., to/from locations) and the cross site transfer line vent station. The main source of information obtained through walkdowns was the condition of the protective features (i.e., the cover blocks) for the transfer line diversion boxes.

\subsection{DATA ASSIMILATION PROCESS}

As noted above, the document review process was accomplished through the combined efforts of numerous individuals in different organizations. It was the responsibility of the team leaders to assimilate all the acquired data into the pre-established table structures. The team leaders participated in all field walkdowns and were responsible for assimilating the field walkdown and interview information into the tables. When multiple sources of information provided different information which could not be resolved, the comment sections were used to identify the discrepancies.

The team leaders were also responsible for reviewing their assigned master checklists of facilities and assuring accountability of all assigned facilities.

All tables were generated in Word Perfect 5.1/5.2 except Tables 6 and 7. Table 6 was generated in Access 2.0 (reports generated with Crystal Report Writer 4.0) and Table 7 was generated in Excel 5.0.

\subsection{REVIEW/SIGN OFF PROCESS}

All tables were sent out for review by the FSAR authors, the cognizant engineers, and the design authorities, as applicable. Meetings were held to discuss comments. All comments received 
were incorporated following these discussions. The tables in Appendices $B$ through $\mathbf{G}$ have been signed-off by the respective SAIC/Stone and Webster team leader who supervised the data assimilation. Upon completion of the formal document review process by WHC and satisfactory incorporation of comments, the respective facility cognizant engineers and design authorities are anticipated to provide final document sign-off. 
This page intentionally left blank. 


\subsection{SUMMARY AND CONCLUSIONS}

The verification activity developed data only for those TWRS facilities within the scope of the present TWRS FSAR, and as such will require additional efforts to develop the equivalent set of data for all respective TWRS related facilities.

The informational data gathered during the course of this effort provides a basis for identifying the variability in the hazards and configurations within TWRS. This data will be used to support the identification and application of a safety management program consistent with the variations within TWRS so that safety management resources can be applied in an effective manner only where required.

The verification project was designed and implemented utilizing existing TWRS documentation, visual inspections of TWRS systems, and current knowledge of TWRS engineering and operational personnel. The result of this effort is an integrated collection of TWRS specific information in one coherent data file which can be used to support three basic TWRS activities:

1) Performance of the analyses and identification of the controls for the TWRS FSAR;

2) Confirmation of facility specific configurations; and

3) Development and/or implementation of a TWRS configuration management program.

To this end, the data has been made available to WHC for input to a relational database to facilitate its utility in these two activities. Once the information has been assimilated, the identification of a smaller subset of safety critical systems, structures and components will be established based on the postulated credible accident scenarios for TWRS. This refined subset of critical systems, structures and components will then require a further detailed field and document verification review to determine for each respective systems items including but not limited to, equipment IDs, review and verification of roundsheets and calibration records, operability assessments and OSR/OSD verifications. This detailed review process for the refined subset of systems will verify the safety basis and associated controls with TWRS facilities.

The information developed by this activity will be turned over to the TWRS design authority for use in a configuration management program. As part of this program it is anticipated that the data will need to be maintained current. Maintenance of the database can be related to routine operational (e.g., round sheets) and engineering activities.

The referenced documentation used as the basis for the development of the matrix tables were in most cases provided by WHC cognizant personnel and was determined to be at the time of review the most appropriate and accurate information available. 
WHC-SD-WM-HIE-007

Rev. 0

This page intentionally left blank. 


\subsection{RTFERENCES}

All documentation used in completion of the matrix tables is listed below. Other reference sources, such as personal interviews, drawings, databases, etc., are not listed here, but are noted on the individual tables located in Appendices B-G.

200 Area Tank Farms Cathodic Protection System Re-Survey, March, 1995.

200 East/West Tank Farms Cathodic Protection System Survey Report, July, 1994.

244-BX DCRT Facility Manual, Stone \& Webster, March 1994

Aging Waste Facility Interim Safety Equipment List, WHC-SD-WM-SEL-020, Westinghouse Hanford Company, Richland, Washington

Aging Waste Interim OSRs, WHC-SD-WM-OSR-004, Westinghouse Hanford Company, Richland Washington.

Authorization Basis Self-Assessment: Requirements Implementation Matrix, WHC-SD-WM-RPT-220, Rev. 0, Westinghouse Hanford Company, Richland, Washington

Development of Radiological Concentrations and Unit Liter Doses for TWRS FSAR Radiological Consequence Calculations, WHC-SD-WM-SARR-037, Rev. 0, Westinghouse Hanford Company, Richland, Washington

Double-Shell Tank Ancillary Equipment Second Containment Evaluation, WHC-SD-WM-EV-040, Rev. 1, Westinghouse Hanford Company, Richland, Washington

Double Shell Tank Composition Status, Leslie Tusler, 74A30-96-008, Westinghouse Hanford Company, Richland, Washington

Double Shell Tank Interim OSRs, WHC-SD-WM-OSR-016, Rev. 0, Westinghouse Hanford Company, Richland, Washington

Double Shell Tanks Interim Safety Equipment List, WHC-SD-WM-SEL-026, Westinghouse Hanford Company, Richland, Washington

Double Shell Underground Waste Storage Tanks Riser Survey, SD-RE-TI-093, Rev. 4, Westinghouse Hanford Company, Richland, Washington

Engineering Study - 200 East Tank Farm Instrumentation Upgrade, WHC-SD-W314A-ES-001, Westinghouse Hanford Company, Richland, Washington

Engineering Study - Diversion Boxes, Environmental Upgrades for Tank Farms, WHC-SD-WM-ES159, Rev. 1A, Westinghouse Hanford Company, Richland, Washington

Final Report Exposure Monitors Data Evaluation for the Hanford High Level Waste Tanks - Tank Farms B, T and TY, Apex Environmental Inc., January 18, 1996 
Hanford Site Tank Farm Facilities ISB, WHC-SD-WM-ISB-001, Rev. 0, Westinghouse Hanford Company, Richland, Washington

Hanford Site Waste Storage Tank Information Notebook, WHC-EP-0625, Westinghouse Hanford Company, Richland, Washington

A History of the 200 Area Tank Farms, WHC-MR-0132, Westinghouse Hanford Company, Richland, Washington

Integrity Assessment Plan for the Double-Contained Receiver Tanks and Designated Ancillary Equipment, WHC-SD-WM-WP-068, Rev. 0, Westinghouse Hanford Company, Richland, Washington

Operational Safety Requirements and Operating Specification Documentation Compliance Instrumentation Matrices - 200 East Area Tank Farm, WHC-SD-WM-EL-003, Rev. 0, Westinghouse Hanford Company, Richland, Washington

Operating Specifications for Aging Waste, OSD-T-151-00017, Rev. D-7, Westinghouse Hanford Company, Richland, Washington.

Operating Specifications for Double-Shell Tanks, OSD-T-151-00007, Rev. H-17, Westinghouse Hanford Company, Richland, Washington

Operating Specifications for Single-Shell Waste Storage Tanks, OSD-T-151-00013, Rev. D-10, Westinghouse Hanford Company, Richland, Washington

Operating Specifications for Tank Farm Leak Detection and Single-Shell Tank Intrusion, OSD-T-15100031, Rev. B-2, Westinghouse Hanford Company, Richland, Washington

Operating Specifications for Watch List Tanks, OSD-T-151-00030, Rev. B-17, Westinghouse Hanford Company, Richland, Washington

Project W-058 Engineering Study, WHC-SD-W058-ES-001, Rev. 0, Westinghouse Hanford Company, Richland, Washington

Project W-314 DST and DCRT Instrument and Control Systems, Initial Assessment, WHC-SD-W314 ES-018, Rev. 0, Westinghouse Hanford Company, Richland, Washington

Project W-314 DST and DCRT Tank Farm Electrical Distribution Systems, Initial Assessment, WHCSD-W314-ES-020, Rev. 0, Westinghouse Hanford Company, Richland, Washington

Project W-314 DST and DCRT Tank Farm Mechanical Systems Initial Assessment, WHC-SD-W314ES-021, Westinghouse Hanford Company, Richland, Washington

Riser Configuration Document for Single-Shell Waste Tanks, SD-RE-TI-053, Rev. 9, Westinghouse Hanford Company, Richland, Washington

Single Shell Tank Leak Stabilization Record, WHC-SD-RE-TI-178, Rev. 5, Westinghouse Hanford Company, Richland, Washington 
Single Shell Tanks Interim Safety Equipment List, WHC-SD-WM-SEL-027, Westinghouse Hanford Company, Richland, Washington

Status Report for Inactive Miscellaneous Underground Storage Tanks at Hanford Site 200 Areas, WHC-EP-0861, Rev. 0, Westinghouse Hanford Company, Richland, Washington

TWRS Transfer Facility Compliance Plan, WHC-SD-WM-EV-094, Rev. 0, Westinghouse Hanford Company, Richland, Washington

Tank Farm Operator Round Sheets AP, AW, Tank Farm and LERF, TF-OR-A-03, Westinghouse Hanford Company, Richland, Washington

Tank Farm Potential Ignition Sources, WHC-SD-WM-ES-362, Rev. 1, Westinghouse Hanford Company, Richland, Washington

Tank Farm Restoration and Upgrades Program Plan, WHC-EP-0392, Westinghouse Hanford Company, Richland, Washington

Tank Waste Remediation System Inactive Miscellaneous Underground Storage Tanks Program Plan, WHC-SD-WM-PD-046, Rev. 0, Westinghouse Hanford Company, Richland, Washington

Transfer Lines, Environmental Upgrade for the Tank Farms, WHC-SD-WM-ES-160, Westinghouse Hanford Company, Richland, Washington

Waste Tank Risers Available for Sampling, WHC-SD-TI-710, Rev. 2, Westinghouse Hanford Company, Richland, Washington

Waste Tank Summary Repor for Month Ending December 31, 1995, WHC-EP-0182-93, Westinghouse Hanford Company, Richland, Washington

Work Plan for Tank Farms, Environmental Upgrades of Ancillary Equipment Secondary Containment, WHC-SD-WM-WP-040, Westinghouse Hanford Company, Richland, Washington 
Rev. 0

This page intentionally left blank. 


\section{MATRIX TABLE INSTRUCTION SHEETS}


Table 1

Materials Inventory Checklist Instructions

This table will be filled out for each tank farm and appropriate miscellaneous facilities.

MAR Category. This column provides the possible types of material which could be located within the tank farms, divided by Material at Risk (MAR) Categories. On page 1 under Tank Contents, list all tanks within the tank farm or miscellaneous building. SARA 313 materials are to be added on page 6 and miscellaneous materials may be added on page 7. If a MAR category does not apply for the tank farm or miscellaneous facility, write NA and proceed to the next MAR category.

NOTE: All information related to tank contents must be provided by J. Sloughter's organization. For single shell tanks, use (1) the December 31, 1995 Hanlon Report, WHC-EP-0182-93. For double shell tanks, use (2) the Tusler report 740A30-96-008.

Note* - For material classification types, use reference \#1 noted above.

Tank headspace data is to be obtained from tank vapor database (TWINS database).

Capacity. The capacity of the tank or vessel should be added here, where applicable. If not applicable, fill in NA. Design capacities to be provided from approved design media. Provide the source of information (e.g., document number).

Material Classification. This column should be completed with information pertaining to the type of material(s) in the unit (e.g., acetylene, acetone, AGING, etc.) Include the source of information (reference document and/or field walk-down). For the tank contents, the following abbreviations should be used:

AGING - Aging waste. High level, first cycle solvent extraction waste from the PUREX plant.

CC - Concentrated Complexant. Concentrated product from the evaporation of dilute complexed waste.

$\mathrm{CP}$ - Concentrated Phosphate Waste. Waste originating from the decontamination of the $\mathrm{N}$ Reactor in the $100 \mathrm{~N}$ Area. Concentration of this waste produces concentrated phosphate waste.

DC - Dilute Complexed Waste. Characterized by a high content of organic carbon including organic complexants. Main sources of DC waste in the double shell tank system are saltwell liquid inventory from single shell tanks.

DN - Dilute Non-Complexed Waste. Low activity waste originating from $\mathrm{T}$ and $\mathrm{S}$ Plants, the 300 and 400 Areas, PUREX facility (decladding supernatant and miscellaneous wastes), 100 N Area (sulfate waste), B Plant, saltwells, and PFP (supernate).

DSS - Double Shell Slurry. Waste that exceeds the sodium aluminate saturation boundary in the evaporator without exceeding receiver tank composition limits. For reporting purposes, DSS is considered a solid.

DSSF - Double Shell Slurry Feed. Waste concentrated just before reaching the sodium aluminate saturation boundary in the evaporator without exceeding receiver tank composition limits. This form is not as concentrated as DSS. 
PD - PUREX decladding. PUREX neutralized cladding removal waste (NCRW) is the solids portion of the PUREX plant neutralized cladding removal waste stream; received in tank farms as a slurry.

PT - PFP Transuranic (TRU) Solids. TRU solids fraction from Plutonium Finishing Plant operations.

Type. Provide the type of waste (e.g., sludge, supernate, etc.) if applicable Provide the source of information.

Physical Form. Indicate if the material is solid, liquid, or vapor.

Volume or Activity. Provide the volume or activity for each material. Include units (gallons, cubic meters, etc) and the source of all information.

Transient. Indicate with a yes/no reply whether the material could be easily moved to another location. For example, a bottle of acetone could be easily relocated or removed and would be considered a transient material. Waste in the double and single shell tanks are not considered transient.

Quantity. Provide the number of containers, if applicable. Include the source of information, if applicable.

Comments. Provide any additional information in this location. Clarify responses as required.

REMINDER: PROVIDE REFERENCES FOR ALL DOCUMENTATION USED.

STATE WHERE WALKDOWN WERE USED TO IDENTIFY MATERIALS (INCLUDE DATE OF WALKDOWN AND NAMES OF INDIVIDUALSI. 
Table 2

Hazard Identification Checklist Instructions

Facility. All "facilities" (the term "facility" is used very broadly here) have been listed by identification number in the first column. While an attempt has been made to create a comprehensive list of the facilities within the given tank farm which will be included in the final safety analysis report, additional facilities may be identified and added to the end of the list.

Type. The type of facility (e.g. single shell tank (SST), double shell tank (DST), valve pit) has been provided in this column.

Columns A-M. The next 13 columns should be completed with information on the hazards which have been identified for each facility. Responses should be coded with numbers using the attached table of responses. The source of information should be provided for each entry. Write NA if the hazards do not apply to the facility.

Comments. Provide any additional information in this location. Additional hazards not covered by the previous categories may be added here. Clarify responses as required.

REMINDER: PROVIDE REFERENCES FOR ALL DOCUMENTATION.

STATE WHERE WALKDOWNS WERE USED TO DOCUMENT HAZARDS IINCLUDE DATE \& NAMES OF INDIVIDUALS RESPONSIBLE FOR WALKDOWN). 


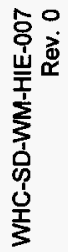

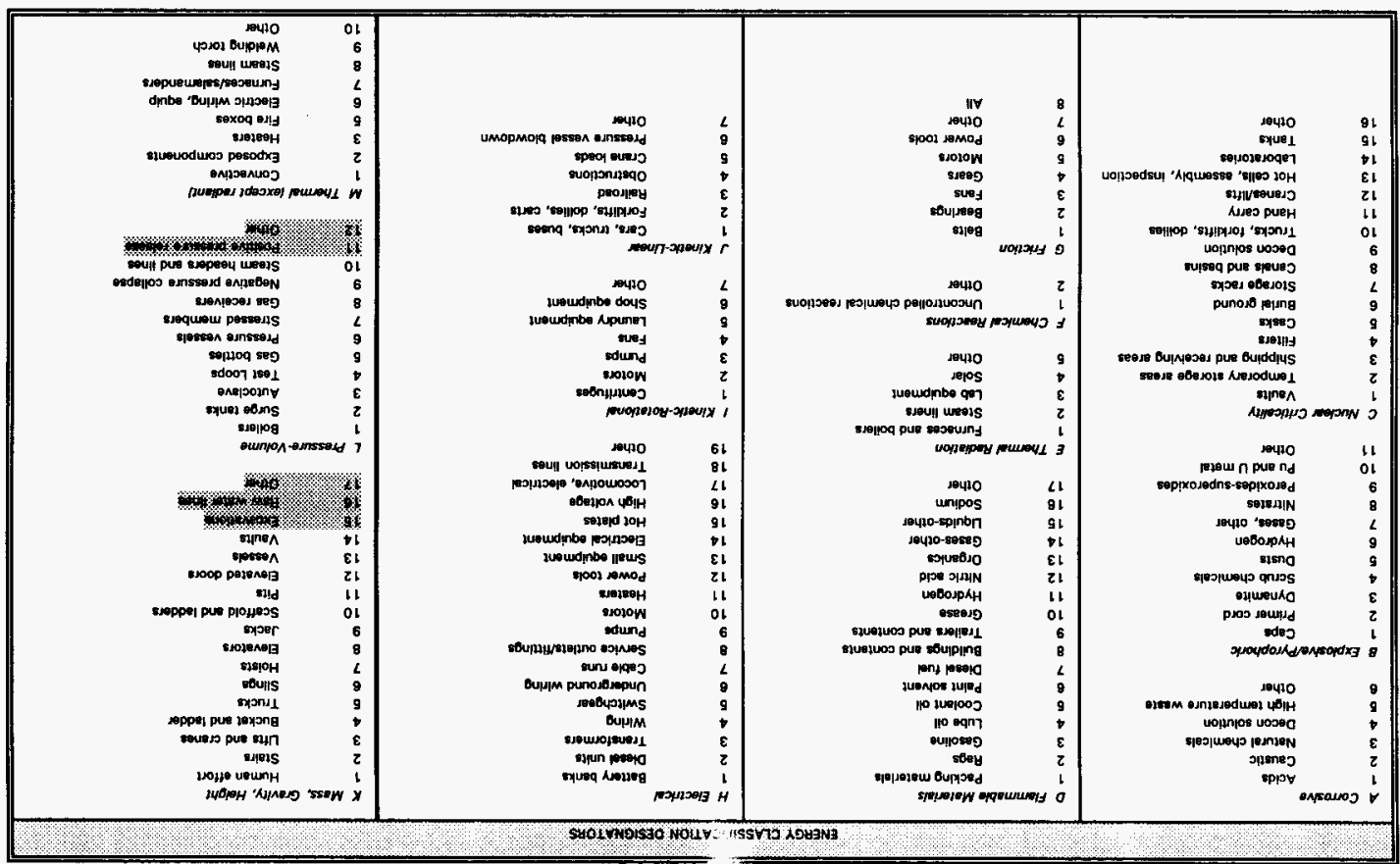


Table 3

\section{Unique Phenomena Checklist Instructions}

Facility. For the purpose of identification of unique phenomena, only DST/SST and DCRTs will be considered.

Type. The type of facility (e.g. single shell tank (SST), double shell tank (DST), double contained receiver tank (DCRT)) has been provided in this column.

The next 7 columns should be completed with information on the potential unique phenomena associated with each facility.

Flammable Gas. Indicate whether flammable gas is present for each facility with a yes/no response. Include the sources of information.

Ferrocyanide. Indicate whether ferrocyanide is present for each facility with a yes/no response. Include the sources of information.

Organics. Indicate whether organics are present for each facility, either as a separable phase, TOC in liquids $(>10 \mathrm{~g} / \mathrm{L})$, or TOC in solids $(>3 \mathrm{wt} \%$ dry basis), with yes/no responses. Include the sources of information.

High Heat. Indicate whether the facility has a high heat source (as defined by the OSDs) with a yes/no response. Include the sources of information.

Pyrophorics. Indicate whether the facility contains potentially pyrophoric material, with a yes/no response. Include the sources of information.

Siphoning. Indicate whether the facility contains a potential siphoning hazard with a yes/no response. Include the sources of information.

Criticality. Indicate if $>1 \mathrm{~g} / \mathrm{L}$ fissionable material exists in the facility. Include the source of information.

Corrosivity. Indicate if the facility contains corrosive materials in excess of the OSD requirements for DST/SST (OSD-T151-00007, OSD-T151-00013). Include the source of information.

Other. List documented discounted events previously evaluated.

Comments. Provide any additional information in this location. Identify any other phenomena for each facility. Clarify responses as required.

REMINDER: PROVIDE REFERENCES FOR ALL DOCUMENTATION.

STATE WHERE WALKDOWNS WERE PERFORMED TO IDENTIFY UNIQUE PHENOMENA. 
Table 4

Facility Configuration Checklist Instructions

System The list of systems contains the type of systems to be reviewed for each tank farm. The system categories include: tanks, pits, boxes, buildings, steam systems, HVAC systems, instrumentation, and transfer lines. For each facility (e.g., tank, pit, box, building etc.) provide the contents (leak detector, CAM, high level alarm, floor drain, etc.). Under buildings, identify the buildings that are associated with the tank farm (a master list is provided for each tank farm, which is probably not up-to-date). For miscellaneous facilities identify the specific facility and list associated components. Add additional facilities at the end of the list provided.

Equipment Identifier This is the system or equipment tag i.d. number or location reference (i.e. pit \#, riser \#, etc.). Provide this i.d. number if it is available.

Equipment Existing Identify if the system or equipment identified in the first column actually exists based on drawing/riser list reviews or field walkdowns. Indicate the source of information (document/drawing number or field walkdown).

Equipment Operable Identify if the equipment/system is currently in operable condition. This information is expected to be obtained through field walkdowns and/or interviews with operators and cognizant engineers.

Equipment Condition This applies to the condition of system equipment. A numeric rating on a 1-10 scale with, 10 being the best, will be provided to individual systems (i.e. ventilation, instrumentation, etc.) as appropriate. For DST systems reference Condition Assessment Surveys for specific facilities (i.e. 241-AW, 241-AP, etc.) If equipment condition is different from the referenced condition documented in the reference report, please note in the "Notes" column.

Notes Use the notes freely, especially for any unusual conditions. Identify exceptions here, e.g., "the leak detector on tank 101 does not work (the leak detectors on other tanks are operable)", "the leak detection pit $03 \mathrm{C}$ serves tanks 101-104 only", etc

REMINDER: PROVIDE REFERENCES FOR ALL DOCUMENTATION USED.

STATE WHERE WALKDOWNS WERE USED FOR VERIFICATION. 
System The list of systems includes locations which are potential radiation sources. If additional sources are identified, add them at the bottom of the list or on an attached sheet.

System ID This is the system or equipment tag i.d. number. Provide this number if it is available.

Radiation Source Identify if the system or location contains a radiation sources or hotspots greater than or equal to $100 \mathrm{Mr} / \mathrm{hr}$ (yes/no answer).

Protective Feature Indicate if the system is or contains radiation protection features. This will include items such as lead shielding, coverblocks, soil over tanks, pits, lines, etc.

Description of Protective Feature Provide a description of the feature (thickness of coverblock, shielding material, etc.) from the drawings or field walkdowns (indicate the source of information). During field walkdowns, note the condition of all visible protective features. Count the number of penetrations on each cover block and approximate

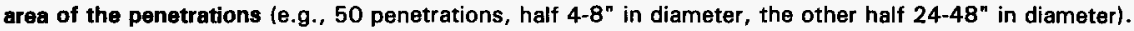

Comments Use the comments freely, especially for any unusual conditions.

REMINDER: PROVIDE REFERENCES FOR ALL DOCUMENTATION.

STATE WHERE WALKDOWNS WERE USED TO OBTAIN INFORMATION. 
Table 6

OSR/OSD Safety Controls Checklist

Systems/Structure/Components. The list of systems contains the systems, structures, and components found on Table 4.

QSR Limits. OSR limits are to be taken from WHC-SD-WM-RPT-220, Rev. 0. Only OSR which were tied to systems, structures, and components in WHC-SD-WM-RPT-220 and are implemented should be reported.

OSD Limits. OSD sections which are tied to systems, structures, and components are to be identified here. OSD sections fufilled by administrative procedures are not to be listed.

Implementing Procedures. List all operating procedures for the associated safety control.

Verification. Indicate if any verification was performed (per WHC-SD-WM-RPT-220). List "DR" for document review (e.g., roundsheet review) and "FV" for anything which was field verfied.

Comments Use the notes freely, especially for any unusual conditions. Note any exceptions to requirements, where applicable. 


\section{Table 7}

Transfer Pipeline Checklist Instructions

Descriptive transfer pipeline data is to be filled out on this table for only active transfer pipelines, which are not within the boundaries of a tank farm. Inactive transfer lines running between tank farms, drain lines, and all transfer lines within individual tank farm boundaries are to be listed on the table by line ID number after the active interfarm lines for FSAR facility configuration purposes only.

Transfer Line ID. Provide the ID number of the active transfer line in this column. Lines which are within the boundary of a tank farm do not need to be included in this table. Segregate lines in the 200 East Area from lines in the 200 West Area.

Size. Specify the diameter of the pipeline in inches.

Type. Specify the type of line (i.e., double encased, concrete encased, direct buried, etc.).

Materials. Specify the transfer pipeline materials of construction.

Capacity. Calculate the pipeline capacity, assuming the pipeline is completely filled with liquid.

Design Pressure. Specify pipeline design pressure.

Connection Facilities. Identify the connection facilities (e.g., diversion boxes) for the pipeline (i.e., to/from locations).

Transfer Frequency. Specify (quantify) the frequency of transfers through this pipeline.

Hydrotesting Frequency. Indicate if the pipe is hydrotested. If yes, specify the frequency of testing.

Leak Detection. Indicate if the pipe has leak detection. If yes, specify the type of leak detection present.

Heat Trace. Specify if the line is heat traced.

Backflow/Siphoning Prevention. Specify if backflow/siphoning prevention is present.

Vent System. Specify if a pipe ventilation system is present. If yes, specify the type of vent system.

Diversion Boxes. Specify if diversion boxes are present. If yes, specify the diversion box identification number.

Cathodic Protection. Specify if the line is cathodically protected.

Comments. Provide any comments needed to clarify responses or provide additional information.

REMINDER: PROVIDE REFERENCES FOR ALL. DOCUMENTATION. 
APPENDIX B

200 EAST AREA DOUBLE-SHELL TANK FARMS

AN, AP, AW, AY, AZ 


\section{AN FARM TABLES}




\section{TWRS FSAR MATRIX TABLE VERIFICATION REVIEW APPROVALS \\ May 31, 1996}

The following information has been assembled. organized, and documented in the attached TWRS Final Safety Analysis Report (FSAR) Matrix Tables in support of Tank Farms FSAR development. Science Applications International Corporation (SAIC), along with the assistance of ICF Kaiser Hanford and the Westinghouse Hanford Company, assembled this data from controlled drawings and databases, supporting documents, as well as other site documentation. Where possible, the data has been field verified by performing field walkdowns of the particular facility of interest. The data also have been reviewed for its completeness and accuracy by the TWRS Facility Operations Design Authority and the Cognizant Engineer assigned to the respective facility. The information, to the best of the knowledge and the time allotted by the undersigned. is true. accurate, and complete as permitted by the referenced documents and drawings shown on the respective tables. No efforts were attempted or made by the authors and/or reviewers to substantiate the reference information shown on the tables beyond the stated qualifiers as noted on the individual facility matrix tables.

\section{AN Tank Farm}

Tank Farm or Facility
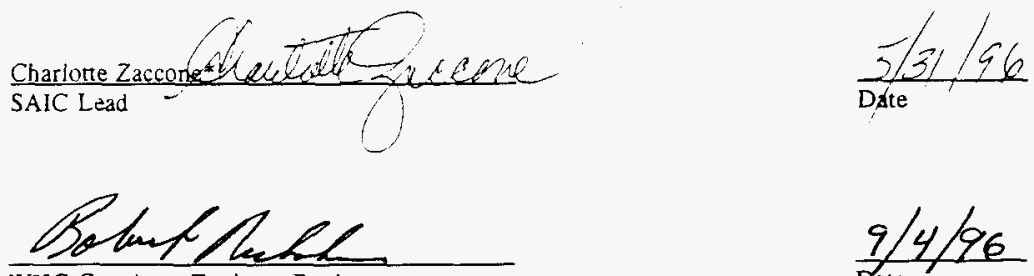

WHC Cognizant Engineer Review
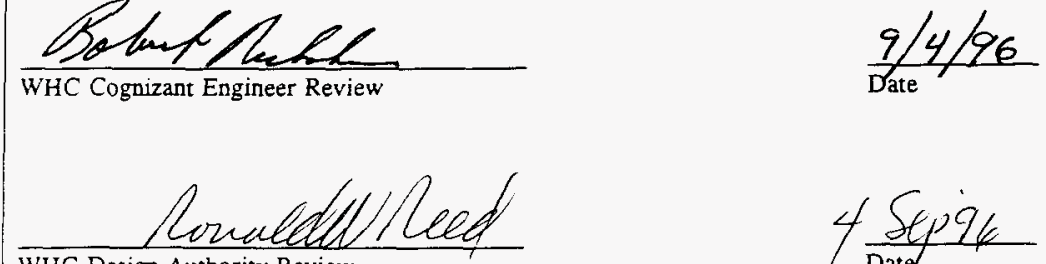

WHC Design Authority Review

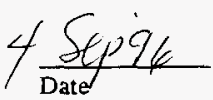

* Stone and Webster support 


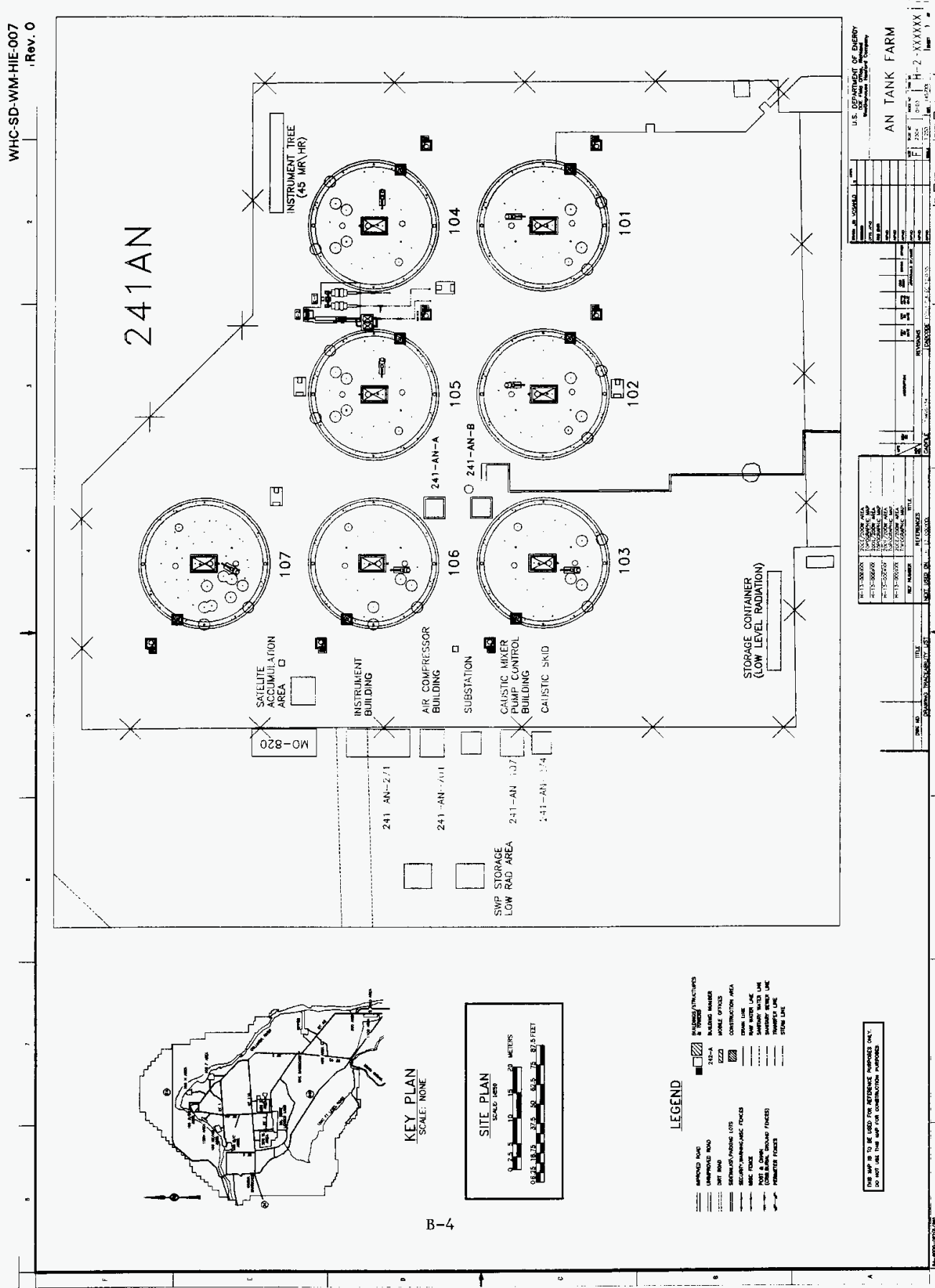


AN FARM

\begin{tabular}{|c|c|c|c|c|c|c|c|c|}
\hline Factlity & Type & $\begin{array}{l}\text { Materiel hrwertory, } \\
\text { (Toble I) }\end{array}$ & $\begin{array}{l}\text { Hazends ID, } \\
\text { (Toble 2) }\end{array}$ & $\begin{array}{c}\text { Unlawe Phenomona, } \\
\text { (Teblo 3) }\end{array}$ & $\begin{array}{c}\text { Facilly Conflouration, } \\
\text { (Tablo 4) }\end{array}$ & $\begin{array}{l}\text { Pud Lovel, } \\
\text { TTablo 5! }\end{array}$ & $\begin{array}{l}\text { Controle, } \\
\text { (Table of }\end{array}$ & Commoent \\
\hline \multicolumn{9}{|c|}{ 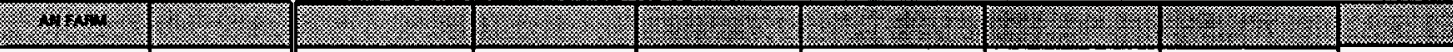 } \\
\hline 241-AN-101 & DST & $x$ & $x$ & $x$ & $x$ & $x$ & $x$ & NA \\
\hline 241-AN-102 & DST & $x$ & $x$ & $x$ & $x$ & $x$ & $x$ & NA \\
\hline 241-AN-103 & DST & $x$ & $x$ & $x$ & $x$ & $x$ & $x$ & NA \\
\hline 241-AN-104 & DST & $x$ & $x$ & $x$ & $x$ & $x$ & $x$ & NA \\
\hline 241-AN-105 & OST & $x$ & $x$ & $x$ & $x$ & $x$ & $x$ & NA \\
\hline 241-AN-106 & OST & $x$ & $x$ & $x$ & $x$ & $x$ & $x$ & ма \\
\hline 241-AN-107 & OST & $x$ & $x$ & $x$ & $x$ & $x$ & $x$ & NA \\
\hline $\operatorname{Cog}-A N-1$ & Cleanout box & $x$ & $x$ & NA & $x$ & $x$ & $x$ & MA \\
\hline COQAN-2 & Cloenour box & $x$ & $x$ & NA & $x$ & $x$ & $x$ & $M A$ \\
\hline COE-AN-3 & Cleenout box & $x$ & $x$ & NA & $x$ & $x$ & $x$ & NA \\
\hline COB-AN-4 & Cleanoun box & $x$ & $x$ & MA & $x$ & $x$ & $x$ & NA \\
\hline COB-AN-5 & Cleenout box & $x$ & $x$ & MA & $x$ & $x$ & $x$ & MA \\
\hline COB-AN-6 & Cleanour box & $x$ & $x$ & MA & $x$ & $x$ & $x$ & $\mathrm{MA}$ \\
\hline COB-AN 7 & Claenour box & $x$ & $x$ & MA & $x$ & $x$ & $x$ & $m$ \\
\hline COB-AN-8 & Cleenour box & $x$ & $x$ & Na & $x$ & $x$ & $x$ & MA \\
\hline COB-ANA & Cleanour box & $x$ & $x$ & MA & $x$ & $x$ & $x$ & MA \\
\hline 241-AN-273 & $\begin{array}{l}\text { Compresser } \\
\text { bulliting }\end{array}$ & NA & $x$ & NA & $x$ & $x$ & $x$ & Ma \\
\hline
\end{tabular}

5/31/96. Mester checklist matrix ostublizhes TWRS FSAR associated facilitios and apacific chockliats for catoporiea 1-6. Check appropriate category for individual tacility checklists (1.0., 241-A-101, material inventory, hazard 10, facility configuration and controls checkfiat is required based on known faclity hazarda.)

NA = Not applicable of not required. 


\begin{tabular}{|c|c|c|c|c|c|c|c|c|}
\hline Fachitity & Type & $\begin{array}{l}\text { Moterial hmentory, } \\
\text { (Table i) }\end{array}$ & $\begin{array}{l}\text { Marand 10, } \\
\text { (Tate 2) }\end{array}$ & $\begin{array}{c}\text { Unique Ptwonomente, } \\
\text { (Tablo 3) }\end{array}$ & $\begin{array}{l}\text { Faclity Complourution, } \\
\text { (Tetos 4) }\end{array}$ & $\begin{array}{l}\text { Pud Lovels } \\
\text { (Tablo o) }\end{array}$ & $\begin{array}{l}\text { Controles, } \\
\text { TTable of }\end{array}$ & Cormment \\
\hline 241-AN & Fiveh pit & $x$ & $x$ & NA & $x$ & $x$ & $x$ & MA \\
\hline 241-AN-271 & $\begin{array}{l}\text { hetrument } \\
\text { bullowing }\end{array}$ & NA & $x$ & MA & $\mathbf{x}$ & $x$ & $x$ & NA \\
\hline & Service pht & $x$ & $x$ & $\mathrm{MA}$ & $x$ & $x$ & $x$ & NA \\
\hline 241-AN-A & Velve olle & $x$ & $x$ & M & $x$ & $x$ & $x$ & NA \\
\hline 241-AN-B & Vetve pht & $x$ & $x$ & MA & $x$ & $x$ & $x$ & NA \\
\hline 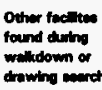 & & & & & & & & \\
\hline $241 \cdot A N-274$ & $\begin{array}{l}\text { Cauntic addition } \\
\text { control bulling }\end{array}$ & $x$ & $x$ & MA & $x$ & $x$ & $x$ & NA \\
\hline & $\begin{array}{l}\text { Caumic adthion } \\
\text { akid }\end{array}$ & $x$ & $x$ & NA & $x$ & $x$ & $x$ & NA \\
\hline & $\begin{array}{l}\text { Sotelite } \\
\text { Aceumulation } \\
\text { Arse }\end{array}$ & $x$ & $x$ & MA & $x$ & $x$ & $x$ & NA \\
\hline & Chenos trater & $x$ & $x$ & MA & $x$ & $x$ & $x$ & MA \\
\hline
\end{tabular}

5/31/98. Master checklist matrix establishes TWAS FSAR associoted facilities and epecific chocklists for cetegories 1-6. Check appropriate category for individual facility checkllats (i.-., 241-A-101, material inventory, hazard ID, faclity configuration and controls checklist is required based on known fectity hazards.)

MA = Not spolicable or not required. 


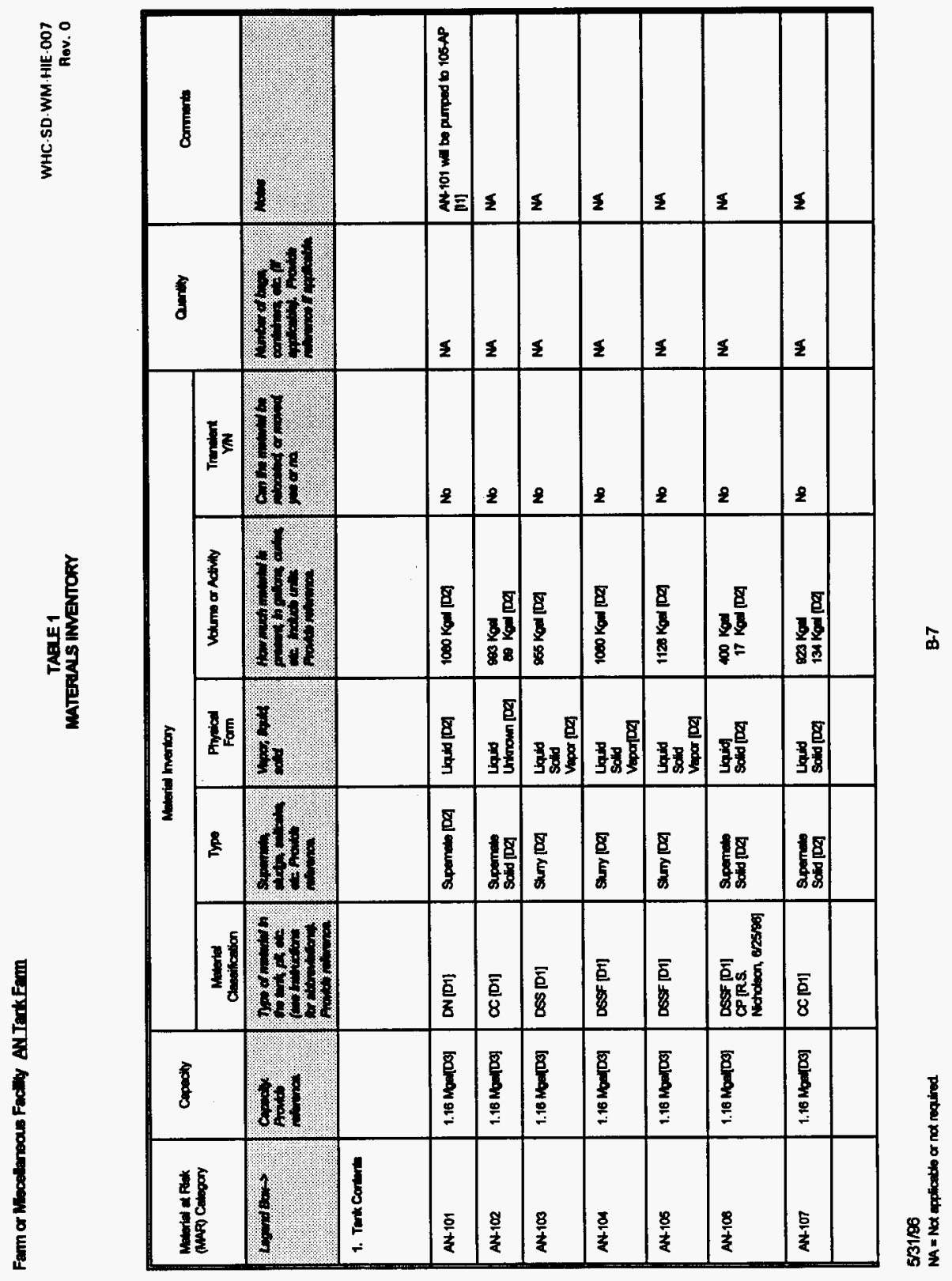


TAEIE 1

\begin{tabular}{|c|c|c|c|c|c|c|c|c|}
\hline \multirow{2}{*}{ 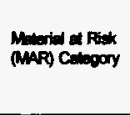 } & \multirow{2}{*}{ Capectly } & \multicolumn{5}{|c|}{ Maveriel invertiony } & \multirow{2}{*}{ andity } & \multirow{2}{*}{ Cormmente } \\
\hline & & ampitiocition & Type & $\begin{array}{l}\text { Phyican } \\
\text { Form }\end{array}$ & Volume or Activy & $\underset{Y \text { Yin }}{\text { Thenent }}$ & & \\
\hline $4 .+10.0=$ & $\frac{c}{40.10}$ & $\frac{19}{4}+1 \%$ & $\frac{1}{4}$ & $4 \%$ & $(1+\infty)+1$ & $\frac{6}{6}+10$ & $\frac{6}{4}$ & 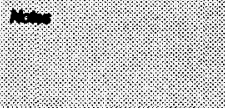 \\
\hline \multicolumn{9}{|c|}{ 2. Terk Headpeos } \\
\hline ANt101 & NA & MA & MA & Vepor & 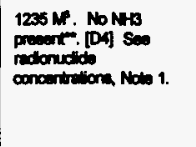 & Yos & ma & 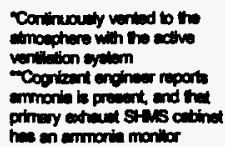 \\
\hline AN-102 & $\mathrm{NA}$ & MA & $M$ & Vapor & 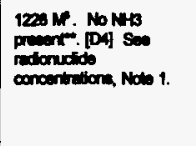 & rer & $m$ & 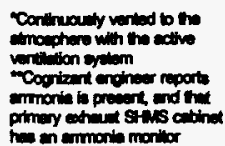 \\
\hline AN103 & NA & MA & $M$ & Vapor & 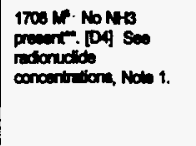 & Yor & MA & 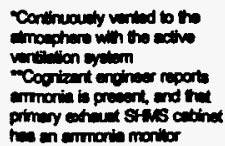 \\
\hline
\end{tabular}




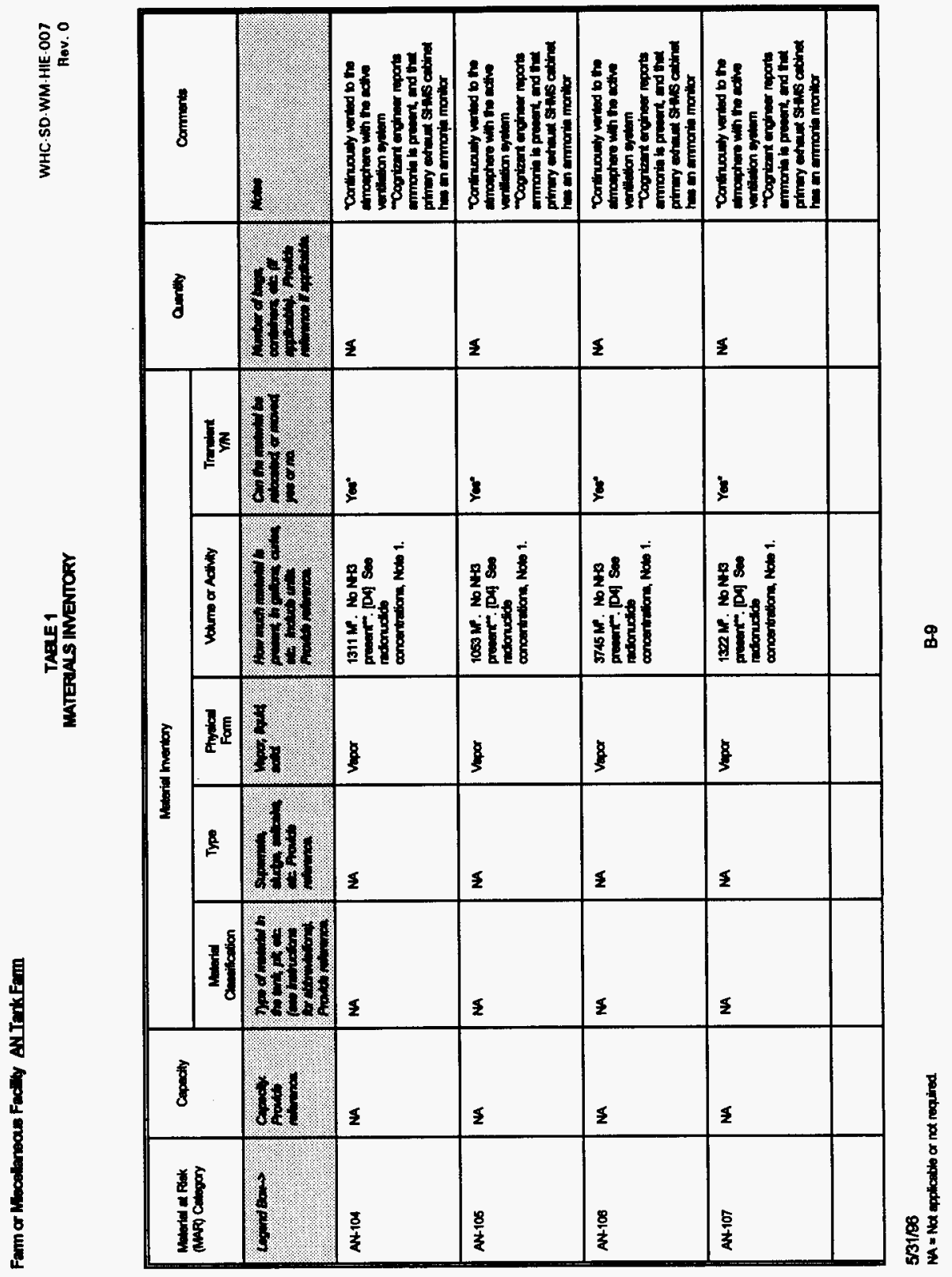




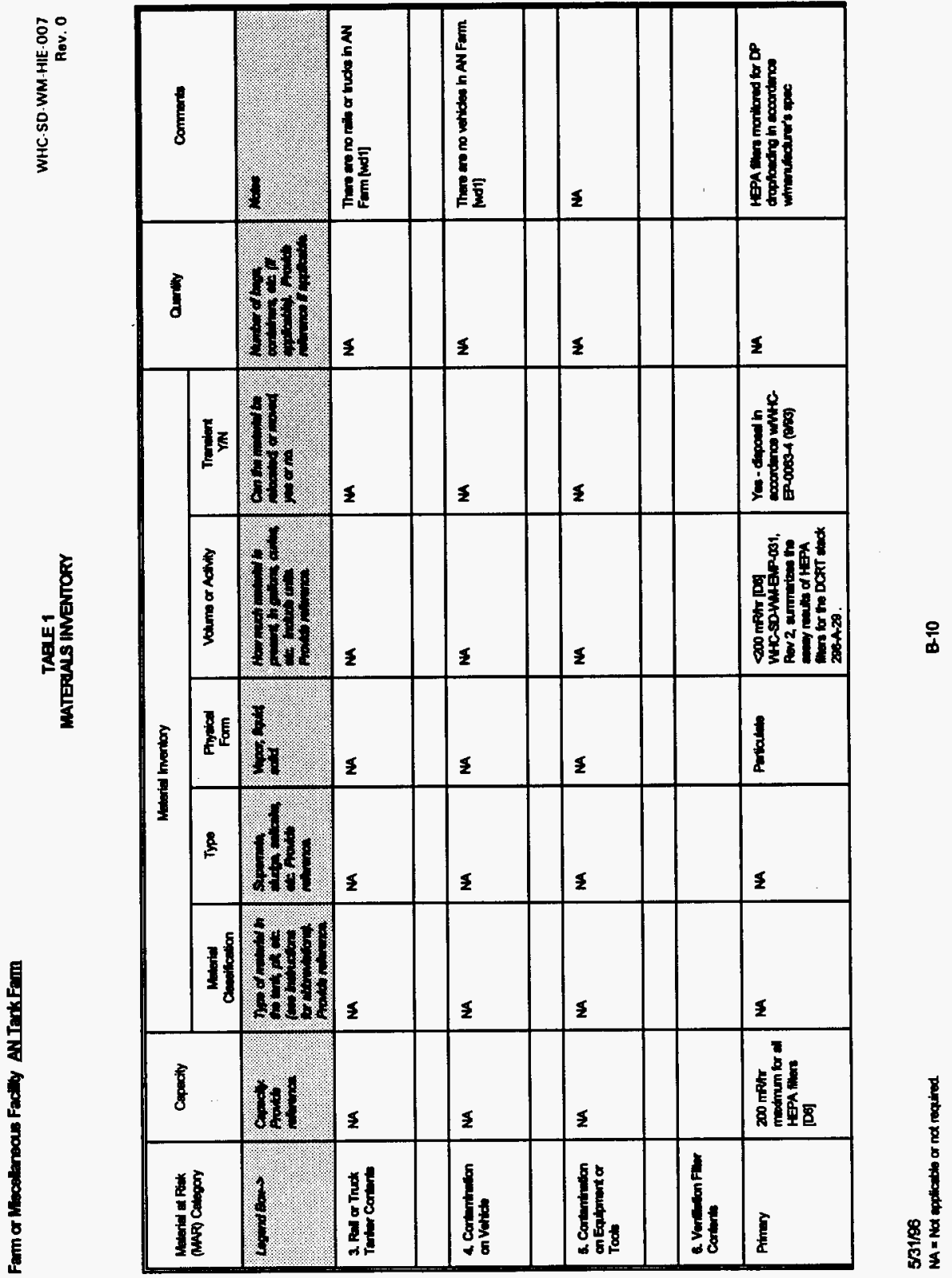




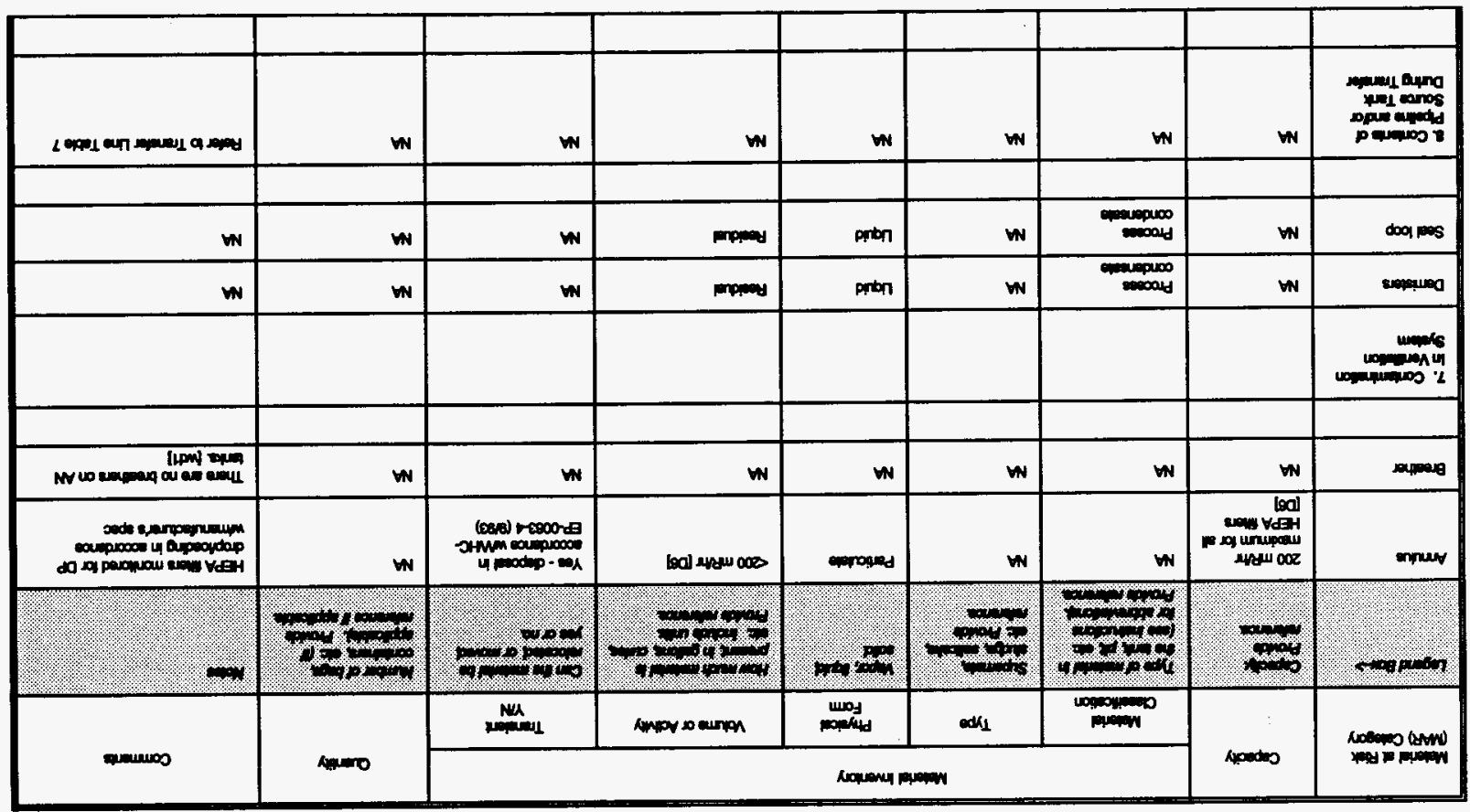

$0 \cdot \mathrm{AOH}$

100-3IH-WM-OS-5HM
NOONENI STREEIW

I IIav1 


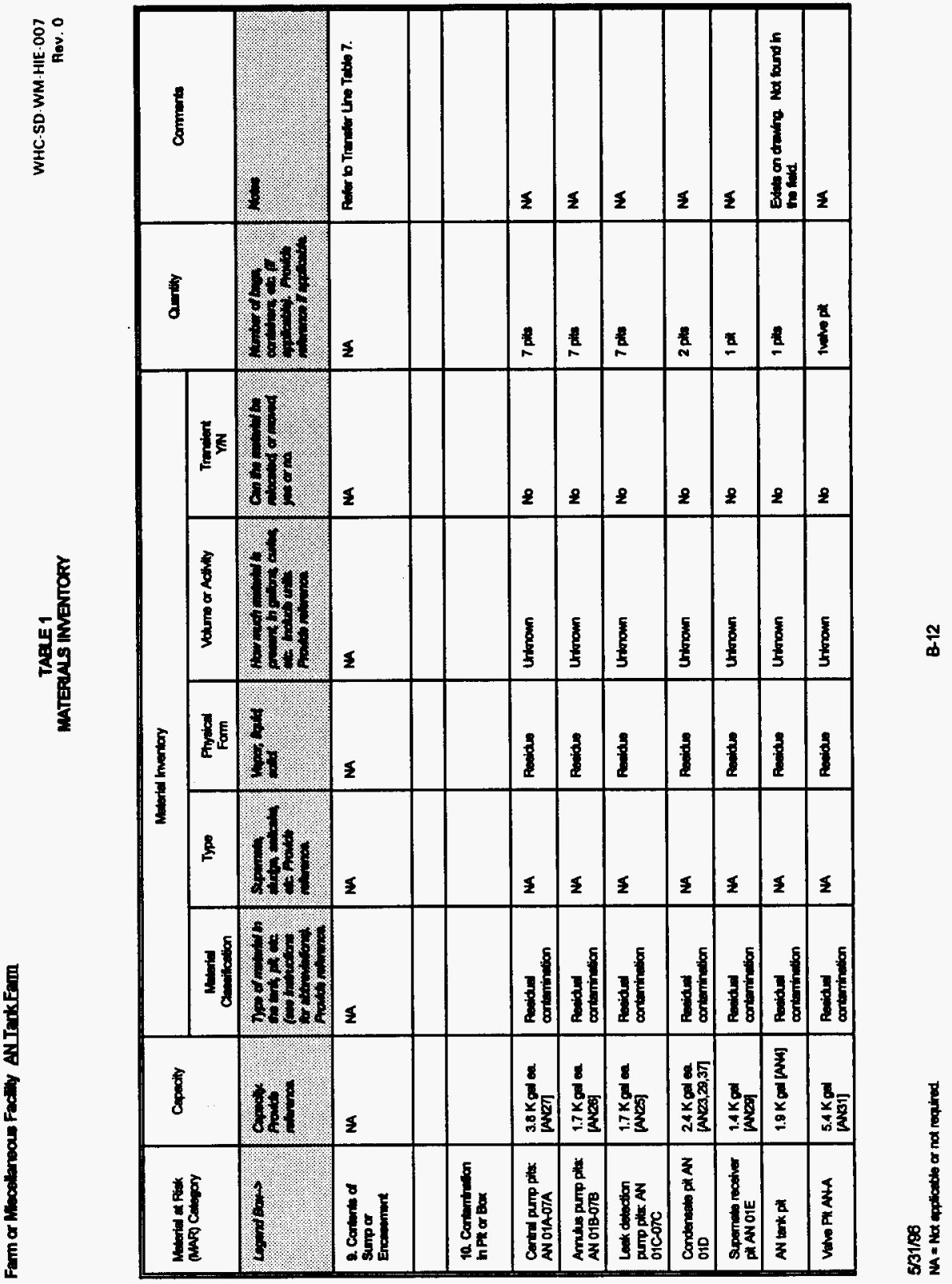




\begin{tabular}{|c|c|c|c|c|c|c|c|c|}
\hline 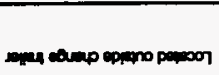 & $\operatorname{mop} x z$ & $\boldsymbol{\omega \lambda}$ & W & $\infty$ & $\mathbf{W}$ & $\begin{array}{r}\text { uctien \%o6 } \\
\text { mapau } \% 01\end{array}$ & Leal $>00 t$ & 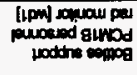 \\
\hline & & & & & & & & ELEvers \\
\hline $\mathbf{W}$ & 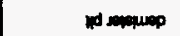 & $\mathbf{9 N}$ & unown & enpiper & $\mathbf{W}$ & 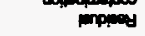 & $\tan 100 \times 66^{\circ}$ & 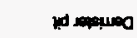 \\
\hline $\mathbf{W}$ & and ant & 9* & moupn & enpery & $\mathbf{w}$ & wotipus & $\begin{array}{l}\text { locand } \\
\text { wox }\end{array}$ & Nond ing \\
\hline $\mathbf{W}$ & $1000 \%$ & $\mathbf{9 N}$ & umospn & mpiost & $\mathbf{w}$ & 10 & [geny] & N $\times 00$ no nomos \\
\hline $\mathbf{W}$ & pat expee i & $\mathbf{W}$ & w & enpoped & $w$ & miving & 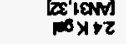 & id cones \\
\hline $\mathbf{W}$ & t & $\mathbf{W}$ & mown & orponed & $\mathbf{W}$ & yopayupos & 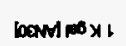 & Hd unt \\
\hline $\mathbf{w}$ & W expl 1 & $\mathbf{W}$ & umopen & enperes & $\mathbf{w}$ & 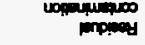 & $\begin{array}{l}\text { IIONy } \\
\text { mxits }\end{array}$ & GeN nd tern \\
\hline 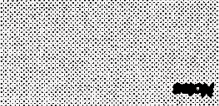 & 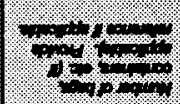 & $(4.6 \%$ & א. & 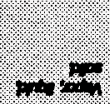 & 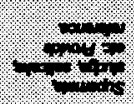 & 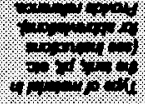 & का & \% \\
\hline \multirow{2}{*}{ Anos } & \multirow{2}{*}{ ONO } & 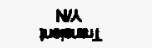 & Amproy do aunon & wost & $\infty K_{1}$ & 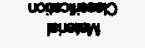 & \multirow{2}{*}{ Apodos } & \multirow{2}{*}{ 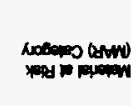 } \\
\hline & & \multicolumn{5}{|c|}{ 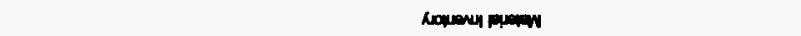 } & & \\
\hline
\end{tabular}

\section{0 noy}

LOO-JHH-WM-OS-JHM
NHONENI STMEIVI

I J1EI 


\begin{tabular}{|c|c|c|c|c|c|c|c|c|}
\hline \multirow{2}{*}{ 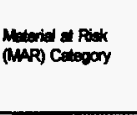 } & \multirow{2}{*}{ Capecily } & \multicolumn{5}{|c|}{ Materies Irvertiony } & \multirow{2}{*}{ aremity } & \multirow{2}{*}{ Comments } \\
\hline & & Manditiontion & Type & $\begin{array}{l}\text { Phycical } \\
\text { Farm }\end{array}$ & Volume or Activity & $\begin{array}{c}\text { Tranient } \\
\text { YWN }\end{array}$ & & \\
\hline wowns & $\cos _{\log }$ & 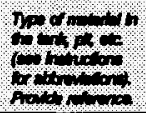 & 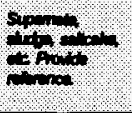 & 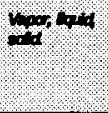 & 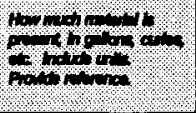 & 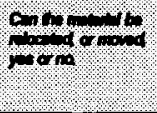 & $\log _{1}$ & now \\
\hline \multicolumn{9}{|l|}{12 Obm } \\
\hline $\begin{array}{l}\text { Coustic adtition } \\
\text { buliding and stid }\end{array}$ & $1 \mathrm{um}$ & See comment & MA & NA & MA & MA & MA & 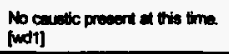 \\
\hline $\begin{array}{l}\text { Sactivite } \\
\text { acosmitation area }\end{array}$ & 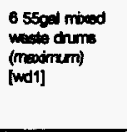 & RCRA Mved & 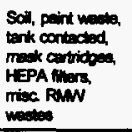 & Solid & 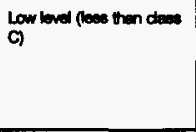 & Yes & $\begin{array}{l}655 \text { oud durme max } \\
\text { capectly for SMA }\end{array}$ & 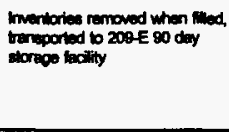 \\
\hline Bulldings & 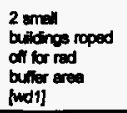 & Low lovel westo & $\begin{array}{l}\text { SWP, other low } \\
\text { ivel woto }\end{array}$ & Solld & Low loved & Yes & $\begin{array}{l}\text { Did not look in } \\
\text { builderos mat they wore } \\
\text { roped of [wd1] }\end{array}$ & 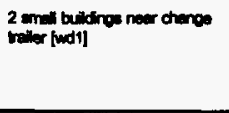 \\
\hline & & & & & & & & \\
\hline
\end{tabular}

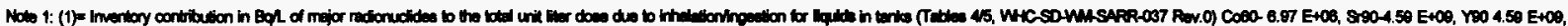

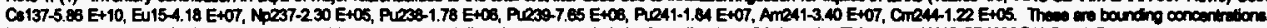

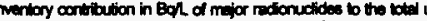

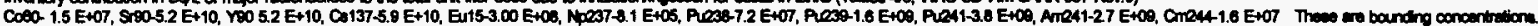

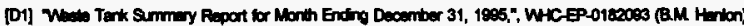

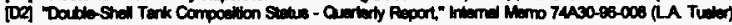

[DO] SD-WASSAR-106, ReN 1

[DA] "TWNS" detane, Jim Huckeby of PNM

[DOI WHCSDWMSARR-037, Rev 0 (oreened by Bil Conloy)

[DE] SDHAKSAR-018, Rov 1

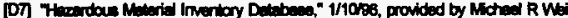

[i1] Comizant Engineer comments, 5/28/98 Bch Nicholson 


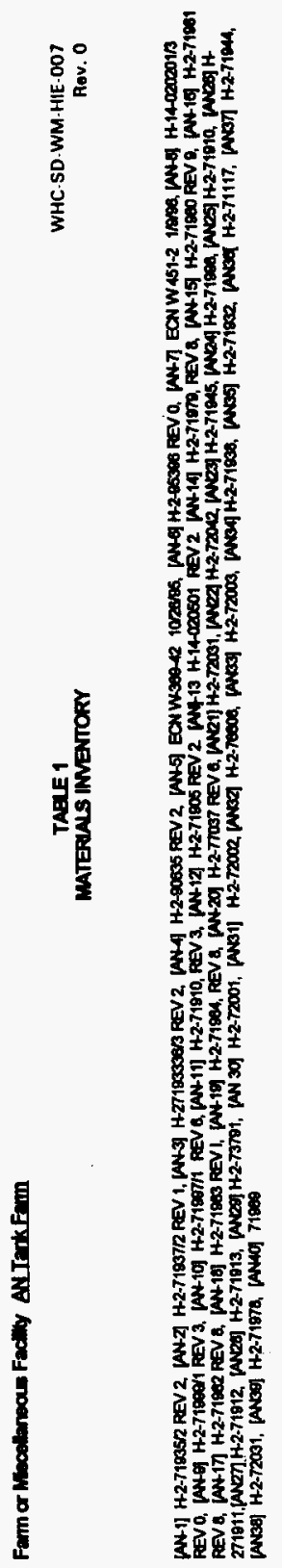

$\frac{n}{d}$

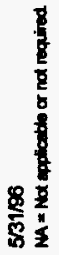


TABLE 2

\begin{tabular}{|c|c|c|c|c|c|c|c|c|c|c|c|c|c|c|c|}
\hline Facilly & Type & Contosive & $\begin{array}{c}\text { Q- } \\
\text { Explotive/ } \\
\text { Pyrophoric }\end{array}$ & $\begin{array}{c}c . \\
\text { Muclay } \\
\text { Criticelity }\end{array}$ & $\begin{array}{c}\text { D. } \\
\text { Flamnivable } \\
\text { Materiale }\end{array}$ & $\begin{array}{l}\text { E- } \\
\text { Thermal } \\
\text { Reciation }\end{array}$ & $\begin{array}{l}\text { F. } \\
\text { Chem. } \\
\text { Rxtre. }\end{array}$ & $\underset{\text { Finction }}{\mathrm{G}-}$ & $\underset{\text { Electricel }}{\mathrm{H}}$ & $\underset{\substack{\text { Kinctio- } \\
\text { Potation }}}{\stackrel{f}{-}}$ & $\begin{array}{l}\text { J- } \\
\text { Khotice } \\
\text { Uneer }\end{array}$ & $\begin{array}{c}\text { K. } \\
\text { Moss, } \\
\text { Grevity, } \\
\text { Holdith }\end{array}$ & $\begin{array}{l}\text { L- } \\
\text { Provine } \\
\text { Votume }\end{array}$ & $\begin{array}{c}\text { Themel } \\
\text { Texceept } \\
\text { redientl) }\end{array}$ & Commente \\
\hline \multicolumn{2}{|c|}{$4 .+\%}$, & \multicolumn{13}{|c|}{ 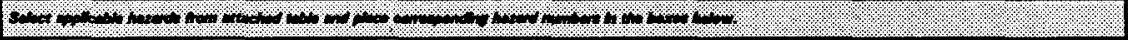 } & $\%$ \\
\hline \multicolumn{2}{|c|}{ AN TANW FAPE } & NA & NA & MA & NA & MA & $\mathrm{Na}$ & 5 (c) & 6 (b) & 2,3 (b) & $m$ & $11(c)$ & NA & ma & NA \\
\hline 241-AN-101 & DST & 2 (b) & $6,7,8 \mathrm{lbl}$ & $15 \mathrm{lbl}$ & 11(b)(1) & NA & NA & $\begin{array}{l}2(b) \mid 31, \\
3(\infty) \mid(b)\end{array}$ & $\begin{array}{l}4,6,9,10 \\
14 \mathrm{lb}]\end{array}$ & $3(b)$ & $(c)(b)$ & 11,15tbit & MA & ma & $\begin{array}{l}\text { I1) Hydrogen not wow } \\
\text { LF }\end{array}$ \\
\hline 241-AN-102 & OST & 2 (b) & $6,7,8 \mathrm{lbl}$ & $15 \mathrm{tbl}$ & $11(b)(1)$ & MA & MA & $\begin{array}{l}2(b)(3) . \\
5,(b) \\
3(b)(b)\end{array}$ & $\begin{array}{l}4,6,9,10 \\
14[\mathrm{bl}\end{array}$ & 3 [b] & (c) $(b)$ & $\begin{array}{l}11.15(b) k \\
c)\end{array}$ & MA & NA & $\begin{array}{l}\text { (2) Armmonte } \\
\text { (3) Pump beoninge }\end{array}$ \\
\hline 241-AN-103 & DST & 2 [b] & $6,7,8$ [b] & $15 \mathrm{lb}$ & $\begin{array}{l}11(b)(1), \\
14(0)(2)\end{array}$ & NA & NA & $\begin{array}{l}2(b)(3), \\
3(d)(b) . \\
7(5) \mid(b)\end{array}$ & $\begin{array}{l}4,6,9,10 \\
\text { if } \\
|b|, 10(6)\end{array}$ & 2,3 lb] & $|c| l|b|$ & $\begin{array}{l}11,15(\mathrm{blt} \\
\mathrm{cl}\end{array}$ & NA & NA & 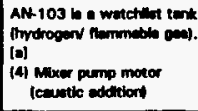 \\
\hline 241-AN-104 & DST & 2 lbj & $8,7,8[b]$ & 15 [b] & $\begin{array}{l}11(b)(1) . \\
14(t)(2)\end{array}$ & MA & NA & $\begin{array}{l}2(b) \mid 3), \\
3(d) \mid(b) . \\
7(b)(b)\end{array}$ & $\begin{array}{l}4,6,9,10 \\
14 \\
(b), 10(0)\end{array}$ & $2,3[b]$ & (c)tos) & $\int_{c \mid}^{11.15(b) t}$ & MA & MA & 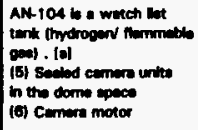 \\
\hline 241-AN-105 & DST & 2 (b) & $6,7,8$ (b) & 15 [b] & $\begin{array}{l}\text { 11(b)(1). } \\
\text { 14|D|(2) }\end{array}$ & NA & $\mathrm{MA}$ & $\begin{array}{l}2(b)(3) \\
3(d)(b) \\
7(5)(b)\end{array}$ & $\begin{array}{l}4,6,9,10 \\
14 \\
(b), 10(0)\end{array}$ & 2,3 [b] & (c) (b) & $\underset{c \mid}{11.15(b) \mid ~}$ & Mn & NA & 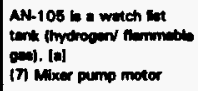 \\
\hline $241-A N-100$ & DST & $\begin{array}{l}2[\mathrm{bb}, 4 \\
\text { [b] }\end{array}$ & $6,7,8[\mathrm{bj}$ & 15 (b) & $11(\mathrm{~b})(1)$ & ma & NA & $\begin{array}{l}2(b) \mid 3), \\
3(d)(b)\end{array}$ & $\begin{array}{l}4,6,9,10 \\
14[\mathrm{~b}]\end{array}$ & 3 [b] & (c) (D) & $\begin{array}{l}11,18 \text { byl } \\
\text { cl }\end{array}$ & $m$ & $\mathrm{MA}$ & NA \\
\hline 244-AN-107, & DST & $2[b]$ & B,7,8 (b) & 15 [b] & $\begin{array}{l}13(b)(1), \\
14(4)(2)\end{array}$ & NA & NA & $\begin{array}{l}2(0) \mid 3), \\
\text { अ(d) } \\
7(5)(b)\end{array}$ & $\begin{array}{l}4,6,9,10 \\
14 \\
\text { (b), 1016) }\end{array}$ & $2,3[b]$ & lettot & $\begin{array}{l}11,15(\mathrm{~b}) \mathrm{l} \\
\mathrm{a}\end{array}$ & $\mathrm{ma}$ & NA & MA \\
\hline $\cos -A n-1$ & Clasenout box & 2 (b) & $6,7,8$ (b] & MA & MA & NA & NA & MA & $4 \mathrm{Ib}]$ & MA & MA & $\mathrm{MA}$ & MA & MA & MA \\
\hline COA-AN-2 & Cloanout box & 2 (B) & $6,7,8[\mathrm{~B}]$ & NA & MA & MA & MA & MA & 4 [b] & $\mathrm{ma}$ & NA & $M A$ & $\mathrm{ma}$ & MA & NA \\
\hline $\cos -A N-3$ & Clemoun box & 2 lb] & $6.7,8 \mathrm{bb}]$ & NA & $m$ & MA & MA & M & 4 [b] & MA & MA & $\mathrm{MA}$ & $M A$ & MA & MA \\
\hline $\cos -A N-4$ & Closmout box & 2 |b] & $6,7,0$ [b] & NA & MA & NA & NA & MA & 4 [b] & MA & MA & $\mathrm{ma}$ & $M$ & Ma & MA \\
\hline
\end{tabular}




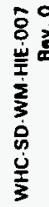

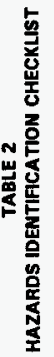

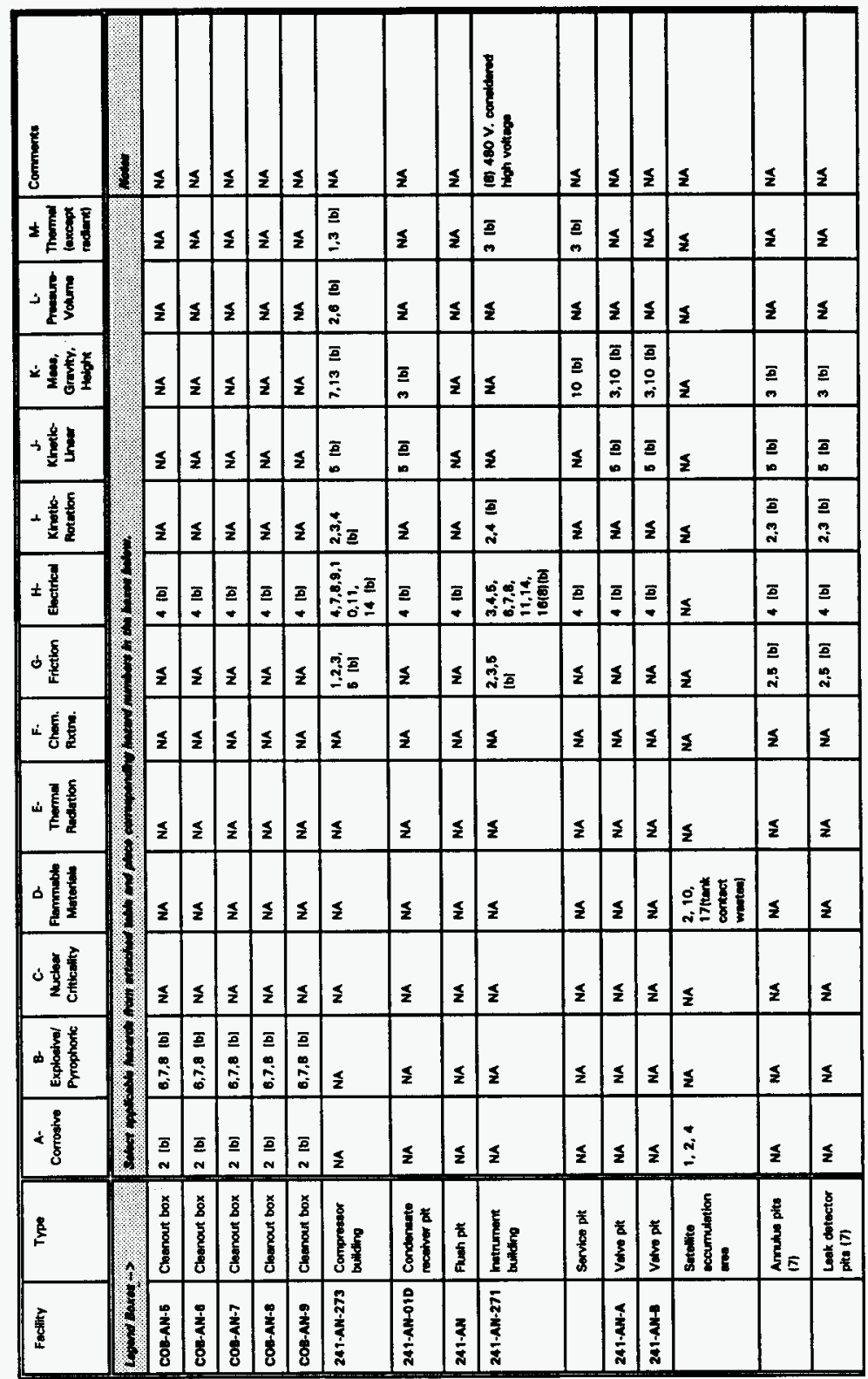

$\frac{7}{\cos }$

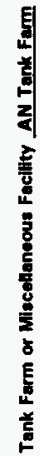

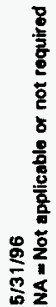




\begin{tabular}{|c|c|c|c|c|c|c|c|c|c|c|c|c|c|c|c|}
\hline Facinty & Type & Corroense & $\begin{array}{c}\text { e- } \\
\text { Explowivel } \\
\text { Pyrophoric }\end{array}$ & $\begin{array}{c}\text { C- } \\
\text { nuciear } \\
\text { Criticallyy }\end{array}$ & Plormmobie & $\begin{array}{l}\text { E- } \\
\text { Therrma: } \\
\text { Rudition }\end{array}$ & $\begin{array}{l}\text { F. } \\
\text { Chom. } \\
\text { Axtron. }\end{array}$ & $\begin{array}{c}G- \\
\text { Friction }\end{array}$ & Eloctricel & $\begin{array}{c}\text { f- } \\
\text { Kinatic- } \\
\text { Rotution }\end{array}$ & $\begin{array}{l}\text { J. } \\
\text { Kinutle- } \\
\text { Innew }\end{array}$ & $\begin{array}{c}\text { K- } \\
\text { Mane, } \\
\text { Grevity, } \\
\text { Heishte }\end{array}$ & 1. & $\begin{array}{l}\text { M. } \\
\text { Thermed } \\
\text { texcepen } \\
\text { neclumel }\end{array}$ & Commenta \\
\hline \multicolumn{2}{|c|}{$10+1,+1$} & \multicolumn{13}{|c|}{ 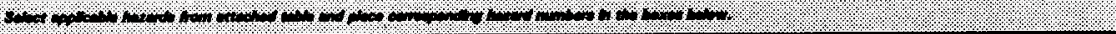 } & $\%$ \\
\hline & $\begin{array}{l}\text { Cemral purno } \\
\text { phe (7) }\end{array}$ & NA & NA & MA & NA & NA & NA & 2,8 [b] & 4 [b] & 2,3 [b] & 5 |b] & 3 [b] & MA & MA & MA \\
\hline \multirow[t]{2}{*}{ 241-AN-01E } & $\begin{array}{l}\text { Supernote } \\
\text { receiver pht } \\
\text { (1) }\end{array}$ & NA & NA & NA & MA & NA & NA & $m$ & 4 (b) & $M$ & 5 (b) & 3 (b) & MA & NA & NA \\
\hline & $\begin{array}{l}\text { Ventilation } \\
\text { pits (4) }\end{array}$ & NA & NA & NA & ma & MA & $\mathrm{MA}$ & $\mathrm{MA}$ & 4 (b) & MA & 5 (b) & 3 [b] & NA & MA & NA \\
\hline \multirow[t]{3}{*}{ 241-AN-274 } & $\begin{array}{l}\text { Conutic } \\
\text { odthion } \\
\text { control } \\
\text { bulling }\end{array}$ & 2 (b) & NA & NA & NA & NA & MA & $2,4[b]$ & $\begin{array}{l}4,9,10 \\
11 \text { lb] }\end{array}$ & 2,3 [b] & MA & NA & NA & $3(9)|b|$ & (9) Heat traces \\
\hline & $\begin{array}{l}\text { Vontilution } \\
\text { pad }\end{array}$ & NA & 6.7 |b| & NA & 11,14 [b] & MA & NA & $\begin{array}{l}1,2,3,4,5 \\
\text { [b] }\end{array}$ & $\begin{array}{l}4,6,10 \\
11,16 \\
|b|\end{array}$ & $2,4[\mathrm{~b}]$ & MA & NA & $\mathrm{ma}$ & 3 [b] & MA \\
\hline & Chenpe traller & MA & NA & MA & NA & $M$ & NA & MA & $\begin{array}{l}4,8,11 \\
\text { (b] }\end{array}$ & MA & $M$ & NA & 5 (b) & $3[b]$ & MA \\
\hline
\end{tabular}

(a) 050-T-151.00030 8-17

(b) COG Engineers: Bob Nichobon, Bil Mooumsen, 4/4/96

(c) Crane loacto in farm, tonk

(d) Component pert of Ventllation eystem 


\begin{tabular}{|c|c|c|c|c|c|c|c|}
\hline \multicolumn{2}{|l|}{$\xi$} & 13 & & 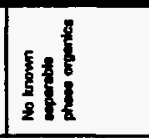 & Eह & 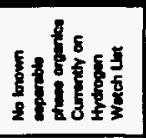 & $\begin{array}{ll}0 \\
5 \\
\end{array}$ \\
\hline \multicolumn{2}{|l|}{$\frac{1}{8}$} & 34149 & & $\frac{5}{2}$ & $\frac{\mathbf{L}}{\mathbf{z}}$ & $\mathbf{s}$ & $\frac{1}{2}$ \\
\hline \multicolumn{2}{|c|}{ 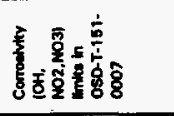 } & $\sqrt[3]{12}$ & & 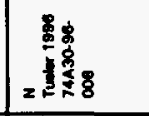 & 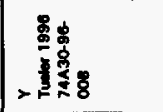 & 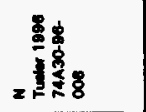 & 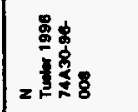 \\
\hline \multicolumn{2}{|c|}{ 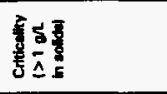 } & 3he & & 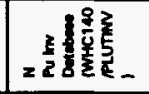 & 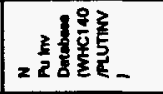 & 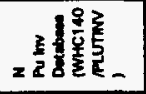 & 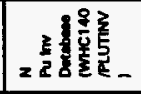 \\
\hline \multicolumn{2}{|l|}{ 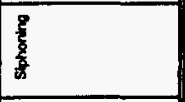 } & in & & 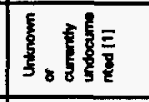 & \begin{tabular}{|l|l|}
5 \\
5 \\
58
\end{tabular} & 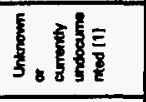 & \begin{tabular}{|l|l|l|l|}
5 \\
5
\end{tabular} \\
\hline \multicolumn{2}{|l|}{8} & 181 & & 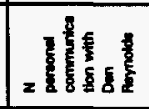 & 28 & 28 & 2 \\
\hline \multicolumn{2}{|c|}{ 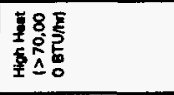 } & If 14 & & × & 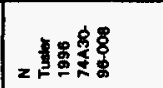 & $\begin{array}{r}1 \\
x \\
x \\
2\end{array}$ & x \\
\hline \multirow{3}{*}{$\frac{8}{8}$} & 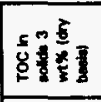 & in & & 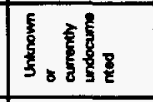 & 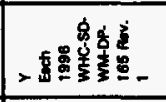 & = & 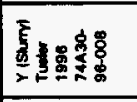 \\
\hline & 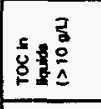 & in? & & 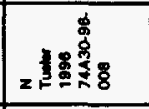 & 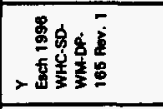 & 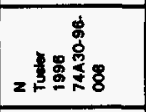 & $\frac{18}{5}$ \\
\hline & 8 & 18 & & 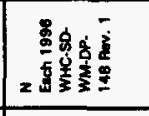 & 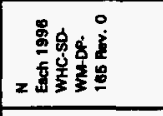 & 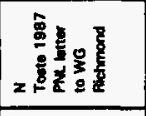 & 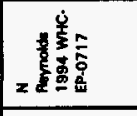 \\
\hline \multicolumn{2}{|c|}{8} & & & 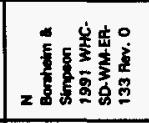 & 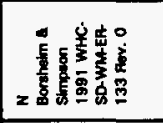 & 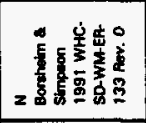 & 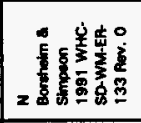 \\
\hline \multicolumn{2}{|c|}{$\frac{8}{5}$} & 3,14 & & 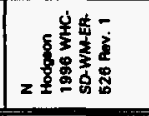 & 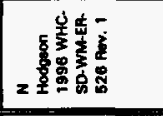 & 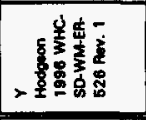 & 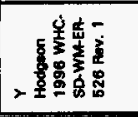 \\
\hline \multicolumn{2}{|l|}{$\xi$} & 1 & & 5 & 吾 & 点 & 5 \\
\hline \multicolumn{2}{|l|}{8} & 3 & $\frac{3}{2}$ & $\frac{2}{2}$ & $\mid \frac{1}{4}$ & $\frac{1}{4}$ & $\frac{1}{3}$ \\
\hline
\end{tabular}


TABLE 3

UWIQUE PHENOMENA CHECKLIST

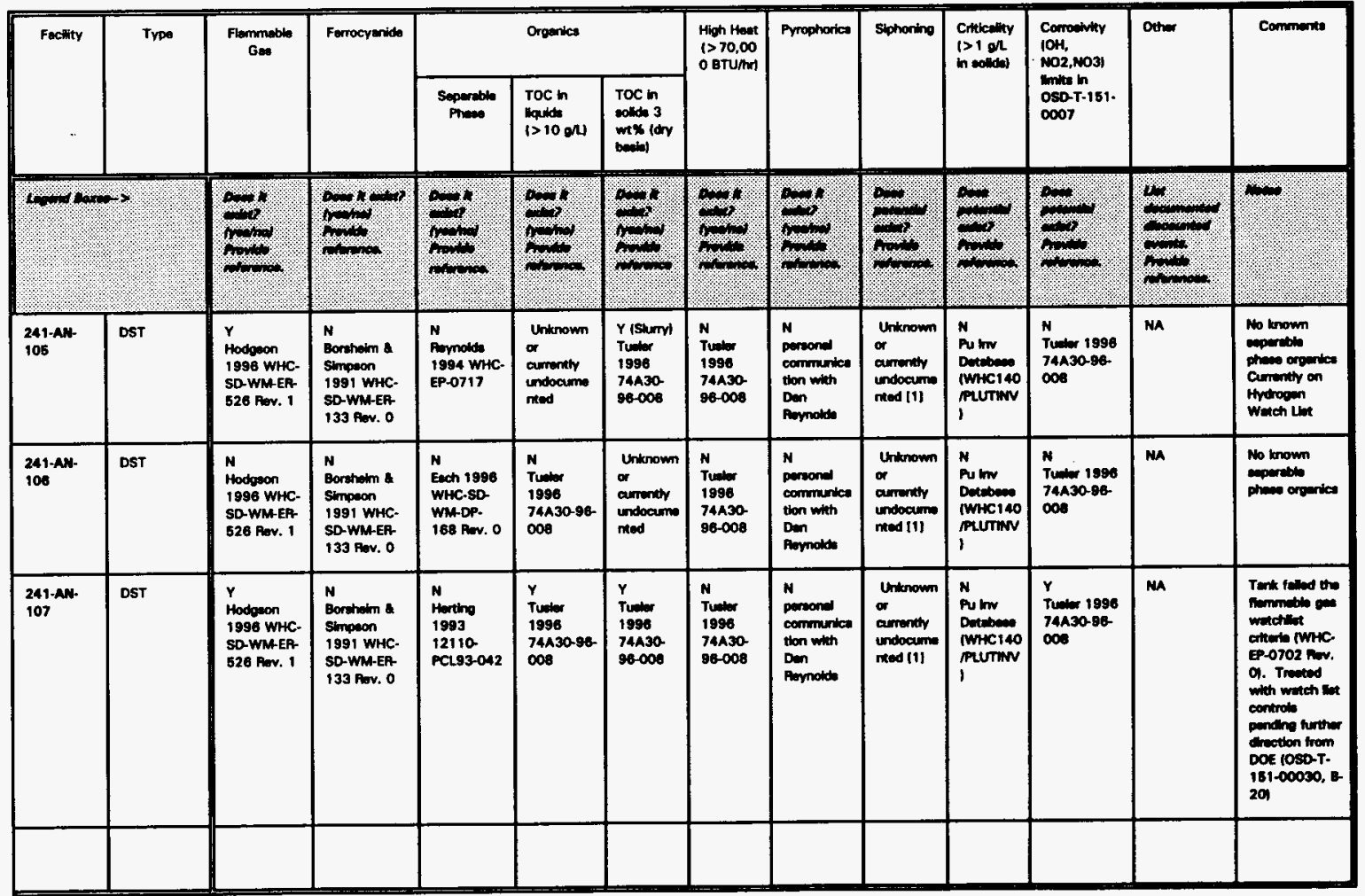




$$
i
$$


TABLE 4

\begin{tabular}{|c|c|c|c|c|c|}
\hline Syatem & $\begin{array}{l}\text { System/ Equipment } \\
\text { Lomitifier }\end{array}$ & Exhating/prusent & Operatio & Equipmert Condition & Comment: \\
\hline 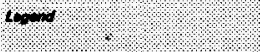 & $0 \% \% \%$ & 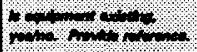 & 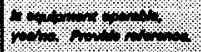 & $(1+\%+1 \%$ & 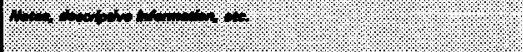 \\
\hline \multicolumn{6}{|l|}{ Tunks AN 101-107 } \\
\hline \multicolumn{6}{|l|}{ Tenk Wats hetrumentation } \\
\hline Specific gravity heotr. syvtem & NA & No & No & MA & wa \\
\hline Temperatures probes IT.C Troal & MA & Yes [md1] (ANI 4-20] & Yea [md1] & MA & MA \\
\hline Llquid lovel (Primerny) & MA & Yos [Wd1] [AN14-20] & Yee [wdI] & MA & 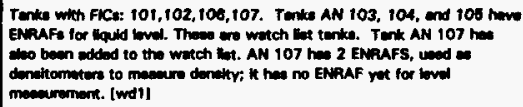 \\
\hline Liquid loved (Beckup) & NA & Yea [wd1] (ANI 4-20] & Yes Imd1] & ma & Menuel tepes. [wd1] \\
\hline High level probe & MA & $Y \in(A N 14-20)$ & Seo comment & NA & 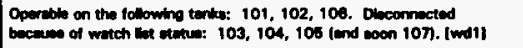 \\
\hline $\begin{array}{l}\text { Tank primary vapor spece proseure } \\
\text { montor }\end{array}$ & NA & Yee [ANA1] & Yes [ANA1] & $\mathrm{MA}$ & MA \\
\hline Ges montoring / SHiMS & AN-103, 104, 105 & Yes [wdIIIANI] & Yee [md1] & MA & NA \\
\hline Mases epectrometer & AN-106 onty & You [md1] [AN7] & $Y e$ [wd1] [AN7] & MA & For gen chercecterlzation \\
\hline TMACS & NA & Yos [wdi] & $Y \in[$ [wd1] & ma & For preacure and tempersture \\
\hline Mulit-tunetion hutrument troe (MIT) & AN-103, 104, 105 & Yoo [md1] & Yes [mdi] & ma & ma \\
\hline TV Comern & AN-103, 104, 105 & Yee [wd1] & $Y=|w d 1|$ & MA & 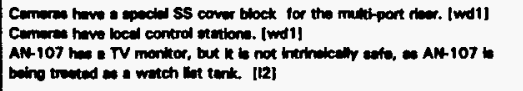 \\
\hline Vepor sampler & AN-103, 104, 105 & Yes [wd1] & See comment & MA & 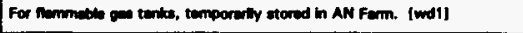 \\
\hline Studgo lovel indicetor & NA & Yec [AN1 4-20] & NA & MA & 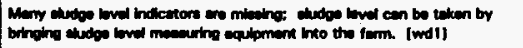 \\
\hline
\end{tabular}




\begin{tabular}{|c|c|c|c|c|c|}
\hline $\mathbf{v N}$ & $\mathbf{v}$ & vw & $\mathbf{Y N}$ & $\mathbf{V N}$ & womposen \\
\hline 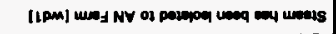 & Vw & $\mathbf{w}$ & VN & VN & nos unsts \\
\hline $\mathbf{w}$ & $\mathbf{v}$ & [LPM] $=1$ & {$[$ [Lm] $\oplus \boldsymbol{\infty}$} & LOLNY & scosprap un iN \\
\hline $\mathbf{w}$ & $\mathbf{w}$ & [Ipm] $\oplus \lambda$ & [Ipm| $\oplus \boldsymbol{\theta}$ & $\mathbf{W}$ & 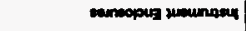 \\
\hline 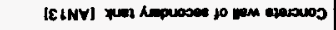 & $\mathbf{w}$ & {$[\varepsilon(+N y]=\alpha$} & 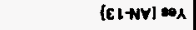 & 40000 & 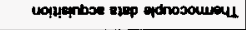 \\
\hline 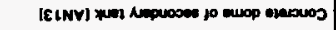 & $\mathbf{w}$ & {$[C[+W \mid=0$,} & $|\varepsilon|+N V \mid=\lambda$ & 400081 & 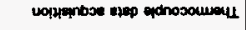 \\
\hline 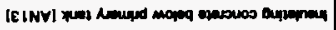 & $\mathbf{m}$ & [C $L+N]=\lambda$ & IE I + NYI $\approx \lambda$ & 40006 & 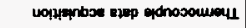 \\
\hline \multicolumn{6}{|c|}{ 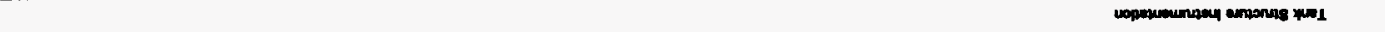 } \\
\hline 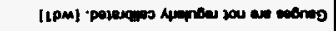 & $\mathbf{W}$ & [LPm] $\oplus$ ג & {$[(p M]=\alpha$} & $\mathbf{V N}$ & 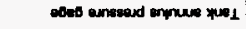 \\
\hline - monosoumen ces & $\mathbf{Y N}$ & $\mathbf{W}$ & $\mathbf{v N}$ & $\mathbf{V N}$ & anyoleduel \\
\hline VN & $\mathbf{V N}$ & ItPMI $=0 \lambda$ & {$[0 Z+I N Y][L P M]=\lambda$} & $\mathbf{v N}$ & 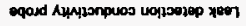 \\
\hline w & VN & [LPM] $1 \mathrm{NA}$ & {$[t p m]=\alpha$} & $\mathbf{V N}$ & uoposep yeer yd dund \\
\hline w & $\mathbf{W}$ & IZเNVI $\boldsymbol{\bullet \boldsymbol { A }}$ & {$[Z+N V]=0 \lambda$} & $\mathbf{V N}$ & ronuow DeH WYS \\
\hline 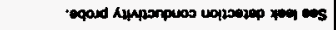 & $\mathbf{W}$ & $\mathbf{w}$ & VN & $\mathbf{v N}$ & edel pnuew uopoejep ypel \\
\hline & & & & \multicolumn{2}{|c|}{ 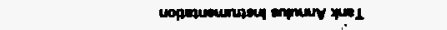 } \\
\hline 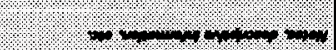 & \%or & 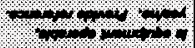 & W. & 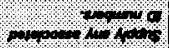 & 1001 \\
\hline stumumos & vopposos werudints & rquedo & hosead/ounserx] & 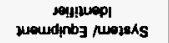 & meje/s \\
\hline
\end{tabular}


TABLE 4

\begin{tabular}{|c|c|c|c|c|c|}
\hline Syatem & $\begin{array}{l}\text { Swrem/ Equipmern } \\
\text { Identifier }\end{array}$ & Exietino/prowent & Operable & Equipmen Condtion & Commente: \\
\hline $100 \%$ & $6 \% \%=\%$ & $6+\%+\%$ & $10 \%+1 \%$ & $6+1 \% 1 \%$ & 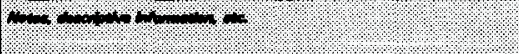 \\
\hline \multicolumn{6}{|l|}{ HVAC syotem } \\
\hline Vonthation Syoten(0) & $K 1, K 2$ & Yee $\{$ wd1] & Yes Imd1I & See cormment & 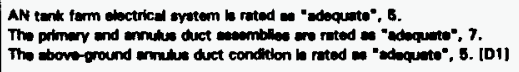 \\
\hline -Pessive & NA & Yos [wd1] & Yea [wd1] & MA & 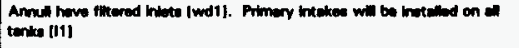 \\
\hline -Activo & Yea [wd1] [AN1] & Yen [11] [wd 1] [ANt] & Yes [11] [wd1] & NA & K1: 2 trins Iwd1] [AN1] K2: 2 traine [wdI] [AN1] \\
\hline $\begin{array}{l}\text {-Shered / common vert evatem? } \\
\text { (Doseribe in comments section) }\end{array}$ & MA & Yes [wd1] [AN1] & Yoo Imdil] [AN1I & MA & 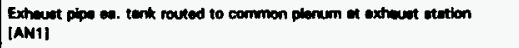 \\
\hline -Exhesust vent/hatedrwory & No [AN1] & No Imdil] & No [md1] & NA & 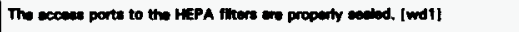 \\
\hline -Arruthe in intake assemblios & $\mathbf{K 2}$ & $Y \in[w d 1]$ & Yeo Ind1) & NA & AN-2 1 HEPA, 1 profintu (AN1) \\
\hline 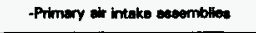 & NA & No [md1] [ANI] & MA & MA & To bo inativilod [11] \\
\hline -Pro-fittere (yos/no) & NA & Yeo [wdi] & Yen [wd1] & MA & MA \\
\hline 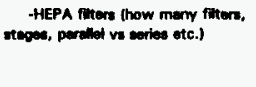 & NA & Yoe (wodi) & Yos [md1] & See comment & 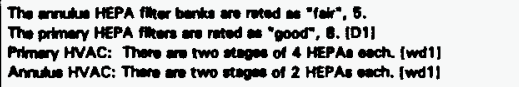 \\
\hline fene & MA & Yea [wdi] & Yee [wd1] & See comment & 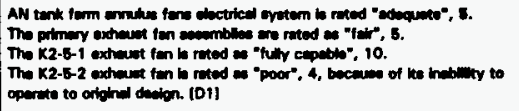 \\
\hline Do-entreinere & NA & Yes [wd1] & Yeo Imdil & NA & $1 \mathrm{cot} \operatorname{tin}[$ [wd1] [AN1] \\
\hline -Dentioter pit & NA & Yes [ANA] & MA & MA & 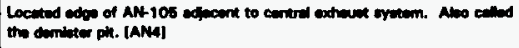 \\
\hline -Houters & NA & Yem [AN1] & Sow cormment & MA & 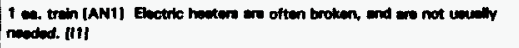 \\
\hline & & & & & \\
\hline
\end{tabular}


TABLE 4

\begin{tabular}{|c|c|c|c|c|c|}
\hline Syatem & $\begin{array}{l}\text { System/ Equpment } \\
\text { Identifior }\end{array}$ & Exiating/prosent & Operation & Equipment Condition & Commente \\
\hline $10 \%$ & 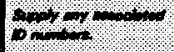 & 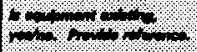 & 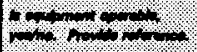 & 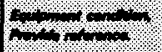 & 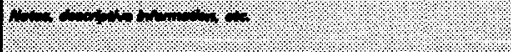 \\
\hline \multicolumn{6}{|l|}{ HVAC hatrumbitation } \\
\hline Main stack moritor & NA & Ye* [wd1] [AN1] & You [wdt] & Na & StMis to be inatallod on the min exhaunter ateck. [wd1] \\
\hline Primery tonk prosume & NA & $Y=$ (wd1) [AN1] & Yos [wd1] [AN1] & MA & Monourned in tenk river. \\
\hline Differential proseure indicaton & NA & Yee [wd1] [AN1] & Yee [wd1] [AN1] & NA & Acroes all HEPA. \\
\hline Temperature indichton: & NA & $\mathrm{r} \in[$ [ANII & Yes (ANI) & $\mathrm{NA}$ & Acroes hewters: \\
\hline Steck flow moter & $\mathbf{K} \mathbf{1}, \mathbf{2}$ & Yee [AN1] & YeA [ANI] & NA & 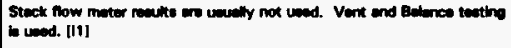 \\
\hline Phinery sead Pot & $\mathrm{M}$ & Yee [AN3] & Yen (AN3) [11] & MA & For ateck phenum conceneate thin lines. \\
\hline -Lever dement & MA & No [AN1,4] & NA & NA & $\mathrm{MA}$ \\
\hline -Drain & NA & Yes $[$ AN1, 4] & Yee & MA & Acumed oporatiolo. \\
\hline Hast traces & NA & $Y \in$ [ANG] & Yee & NA & Aewimed operiblo. \\
\hline Anulus seat Pot Fh & MA & Yeo [AN1,4] & Yeo IAN1,4I III] & NA & NA \\
\hline -Loved etsernerit & NA & No [AN4] & MA & NA & MA \\
\hline - Drah & NA & $Y=0$ (ANA) & $Y=$ & MA & Anermed operiblo. \\
\hline \multicolumn{6}{|l|}{ Ventifition $\mathrm{m}$} \\
\hline Veritiotion Pht $=1$ & AN 102,103 & Yas [II] [ANB] & Yes [ANG] [11] & NA & Ma \\
\hline - Armitus duct preacurns & NA & Yes [ANB] & Yes [ANE] [11] & MA & Ma \\
\hline - CAM & NA & Yea [ANB] & Yas [ANE) [II] & MA & Anoulinin lonkt detection CAM. \\
\hline Flow control valve & NA & Ya [ANB] & Yen (ANME) [II] & ma & MA \\
\hline
\end{tabular}




\begin{tabular}{|c|c|c|c|c|c|}
\hline wasedo peuners & $w$ & $\omega$ & [GNV] అ八 & $\mathbf{V N}$ & Dup Aends nom- \\
\hline vw & $\mathbf{v}$ & 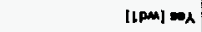 & [GNY] ILPen] $=0$ & $\mathbf{V N}$ & volpopsep yer. \\
\hline w & $\mathbf{W}$ & $\mathbf{v N}$ & [GNYI ON & VN & deurns- \\
\hline $\mathbf{W}$ & $\mathbf{V N}$ & ItII $=\mathrm{A}$ & IENVI $=\lambda$ & $\mathbf{w}$ & chep noots \\
\hline $\mathbf{V N}$ & $\mathbf{V N}$ & ItII $\omega_{\lambda}$ & $[1]] \approx 0$ 시 & $\mathbf{V N}$ & 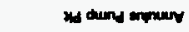 \\
\hline & & & & & and \\
\hline 'vapuado pournedy & $\mathbf{w}$ & $\infty_{\Lambda}$ & {$\left[Z Z^{\prime} 1 Z \mathrm{NV}\right]=0 \mathrm{\lambda}$} & 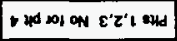 & mpar - \\
\hline & & & & & HA wourvasul wopengues \\
\hline $\mathbf{w}$ & $\mathbf{w}$ & [LI] [ONYI $=0$ ג & IENVI EN & $\mathbf{w}$ & expen paypor moy- \\
\hline $\mathbf{w}$ & $\mathbf{w}$ & [LI] [BNV] wA & [BNV] 61 & $\mathbf{W}$ & พvo- \\
\hline Wvo uopsosep wem mpaury & vw & (III) IBNYI $10 \mathrm{~A}$ & [QNY] & VN & 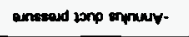 \\
\hline $\mathbf{w}$ & VN & III) lanVI $\infty \mathrm{A}$ & IENV] & $\angle O L N Y$ & 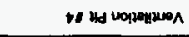 \\
\hline $\mathbf{w}$ & VN & ILI] [ENYI कx & IENYI $=1$ & $\mathbf{V N}$ & axpen papuos molj. \\
\hline 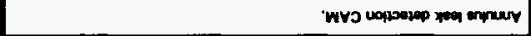 & vw & [III lanVI ex & [ENYI $\infty_{\lambda}$ & $V N$ & wvo- \\
\hline VN & $\mathbf{V N}$ & ILI] lenvi $\infty \mathrm{X}$ & [BNY] అx & $\mathbf{V N}$ & anepand f3ro munuy- \\
\hline $\mathbf{w}$ & $\mathrm{VN}$ & [III ISNYI $=1$ ג & [BNY] ENA & SOL'SOL-NY & E) मd Lompinuan \\
\hline $\mathbf{w}$ & $\mathbf{V N}$ & ILII IENYI $\oplus_{A}$ & [ENV] $\approx \lambda$ & VN & anpan poswos moYs. \\
\hline 'Wvo uon30uep yoe mpany & $\mathbf{V N}$ & [iI] [gNYI & [ENY] & $\mathbf{V N}$ & พทว- \\
\hline \multirow[t]{2}{*}{$w$} & $m$ & [LH] leNY) W1 & [ENV] $=1$ & $\mathbf{V N}$ & 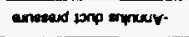 \\
\hline & $\mathrm{w}$ & (LI) IENYI Do & ENVI $\oplus \mathrm{A}$ & TOL'LOLNY & Co Ha Uopaptiven \\
\hline $4 \%$ & (1) & Whor & 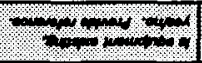 & $1,+\infty \%$ & 1,17 \\
\hline overumas & uspypuos meundritog & apenodo & 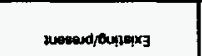 & 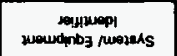 & unoteds \\
\hline
\end{tabular}

\section{$0.20 \mathrm{H}$}

COO JIH.WM OS-כHM

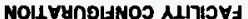




\begin{tabular}{|c|c|c|c|c|c|}
\hline Syetem & $\begin{array}{l}\text { System/ Equpmant } \\
\text { hientifier }\end{array}$ & Exleting/present & Operables & Equiprnent Condition & Commente \\
\hline 200 & 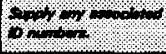 & 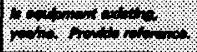 & 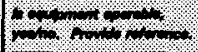 & $6 \% 1 \%$ & 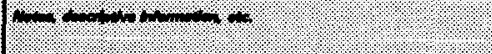 \\
\hline Conterd Punp $\mathrm{Ft}$ & NA & $Y e n[1]\}]$ wod 1] & You IIII & MA & MA \\
\hline -Lak dotection & NA & | Yen Iwd1] [AN23] & Yed IwdII & $\mathrm{m}$ & NA \\
\hline - Floos drentin & NA & YoE [AN24] & $Y=0$ & MA & Aseumed operable. \\
\hline -Check Valvo & NA & Y Yes [ANR24] & Ye & NA & Acoumed opernatis. \\
\hline Portoble ph epray (1. RW) & NA & You IAN24I & Yen & NA & Aceumed operable. \\
\hline Dinthutor Ph & NA & NA & HA & $\mathbf{M a}$ & $\mathbf{~ N A}$ \\
\hline mica $\mathrm{Ph}$ & NA & MA & MA & NA & NA \\
\hline Heal Ph & MA & No & $m$ & Ma & $\mathrm{ma}$ \\
\hline Lonk detectiton purnip ph & NA & Yee lII] & You [II] & MA & $\mathbf{M a}$ \\
\hline -Lovet sencing system (SpG-WF sump) & NA & Ves (wd1] [AN13) & $\begin{array}{l}\text { Yen, waceopt for AN-103 } \\
\text { [wd]I] }\end{array}$ & NA & 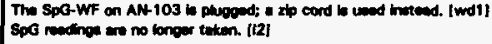 \\
\hline - Rudiation monitor (mump) & NA & Yes [AN13] [II] & Yos & ma & Anoumes operath. \\
\hline -Leak dotoction syetem (ph) & NA & $Y \in=\mid$ (wd1) [AN13) & Yos [wdt] & MA & ma \\
\hline -Temperature & NA & YoE [ANIOI & Yes [mdi] & MA & Tomp. woll in leat ph (AN1O) \\
\hline -Well & NA & YeE (AN13) & Yos & NA & Asoumned operablo. \\
\hline -Drain & NA & Yes [ANIO] & Ye: & NA & Asoumed operablo. \\
\hline
\end{tabular}




\begin{tabular}{|c|c|c|c|c|c|}
\hline $\mathbf{w}$ & $\mathbf{w}$ & VN & $\mathbf{V}$ & w & 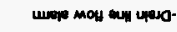 \\
\hline $\mathbf{w}$ & $\mathbf{w}$ & $\mathbf{w}$ & $\mathbf{v}$ & $\mathbf{V}$ & moxoums anneary- \\
\hline 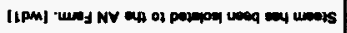 & $\mathbf{w}$ & $\mathbf{W}$ & [LPM] on & VN & weass- \\
\hline acenodo peuniver & $\mathbf{w}$ & $\omega_{x}$ & IGENVI) $\propto$ & $\mathbf{W}$ & una- \\
\hline w & w & VN & $\mathbf{w}$ & w & ayponow moll armoded. \\
\hline 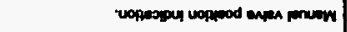 & $\mathbf{w}$ & $\|$ thl $\mathbf{m a n}$ & [1] $\operatorname{ens}$ & VN & 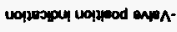 \\
\hline$w$ & $w$ & [Lрм| $ш$ A & I6E' LE'EZNYI ILPM] mA & $\mathbf{V N}$ & volparep yeor- \\
\hline w & $\mathbf{w}$ & {$[$ [ $\mathrm{pm}] \boldsymbol{\infty \Lambda}$} & {$[L \mathrm{DM}]$ [LII $\operatorname{\infty os}$} & any $\forall+n v$ & (z) and enpa \\
\hline 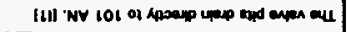 & $m$ & $\mathbf{w}$ & Itill tLOM] ON & $\mathbf{W}$ & wat unag \\
\hline$m$ & $\mathbf{w}$ & $w$ & on & $w$ & 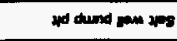 \\
\hline n & $\mathbf{w}$ & $\mathbf{w}$ & on & VN & Wd dund copns \\
\hline $\mathbf{w}$ & $\mathbf{v w}$ & {$\left[\right.$ Ipm] [L]] $\boldsymbol{\infty}_{\lambda}$} & 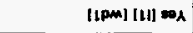 & VN & vo1290190 ye07- \\
\hline [lam] $101 \mathrm{NV}$ พwe 1 wo posesol & $\mathbf{w}$ & I Ipm] $\boldsymbol{\omega}_{\lambda}$ & [IPM] $=0$ A & 010 & 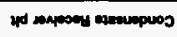 \\
\hline 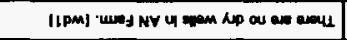 & $\overrightarrow{V N}$ & VN & VN & VN & $\operatorname{sen} 10$ \\
\hline eqpedo pecuneor & $\mathbf{w}$ & $\infty_{\lambda}$ & [OINVI $\mathbf{~ D A}$ & $\mathbf{W}$ & Ande neass. \\
\hline \%., & (1) & $\%$ & 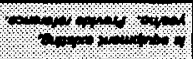 & 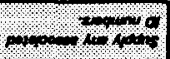 & 101 \\
\hline axuminos & 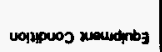 & 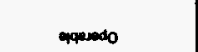 & 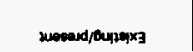 & 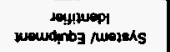 & worels \\
\hline
\end{tabular}

o ney

100. ZlH-WM OS-JHM

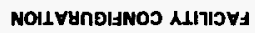

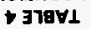


TABLE 4

FACILITY CONFIOURATION

WHC-SO-WM-HIE-007

Rov. 0

\begin{tabular}{|c|c|c|c|c|c|}
\hline System & $\begin{array}{l}\text { Systern/ Equipment } \\
\text { Identifier }\end{array}$ & Exbsting/present & Opereble & Equipment Conchition & Commenta \\
\hline 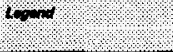 & $10 \%+1 \%$ & 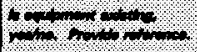 & 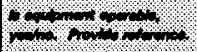 & Sor, & 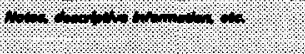 \\
\hline crem oun Boxes & $\cos 1-9$ [wd1] & Yos [wd1] & Yoe IndII] & NA & SAR Ch 2 thaw $\theta$ COB \\
\hline Loak Dotection & MA & Yes [Wd1] [AN23,39] & ree (wdI) & MA & MA \\
\hline Fand Purrep Pa & NA & Mo & NA & MA & NA \\
\hline Mixer Pump Pt & NA & MA & NA & MA & MA \\
\hline Sorvices $\mathbf{m}$ & MA & Yoe $[111$ Indil & 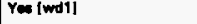 & NA & MA \\
\hline - Flow monitoring & NA & Yee [Wd1I [AN37] & Yes Imdt] & $\mathbf{M A}$ & MA \\
\hline - Red montior & NA & Yes [md1] [AN37] & Yeo [wodi] & NA & MA \\
\hline -Backilow preverter & NA & Yes Imdl] & Yes IwdI] & $\mathrm{m}$ & MA \\
\hline - Loak donection & MA & Yes [Wd1] [AN37] & Yee [woll] & M & MA \\
\hline -Proceure Geupos & NA & Yes [md1] [AN37] & Yeo Imotl] & MA & $\mathbf{M}$ \\
\hline -sump & NA & No [wd1] [AN30] & Ab [wd1 [AN3O] & MA & MA \\
\hline -Drain & MM & Yeo [ndil] & $Y \omega$ & M & Anumad operiblo. \\
\hline . Sump bovel eloment & NA & No (AN3O) & No & MA & Aloumed operiblo. \\
\hline - Hooter & NA & $M$ & MA & MA & MA \\
\hline Fush pot & 241-AN & $Y=0$ & You & NA & MA \\
\hline Flow monitoring & MA & NA & MA & NA & MA \\
\hline
\end{tabular}

\section{$5 / 31 / 96$}

NA $=$ Not appilcablo or not nequired 


\begin{tabular}{|c|c|c|c|c|c|}
\hline $\mathbf{w}$ & $\mathbf{w}$ & (LPM] $\oplus \lambda$ & (IPM) $=0$ & $m$ & $\operatorname{selpay-}$ \\
\hline$m$ & $w$ & [IPm] $\cos _{\lambda}$ & 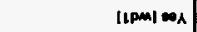 & $\mathbf{~} \mathbf{N}$ & wint seapeoser - \\
\hline $\mathbf{W}$ & $\mathbf{w}$ & $\mathbf{w}$ & $\mathbf{V N}$ & $\mathbf{v N}$ & 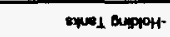 \\
\hline 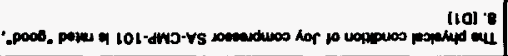 & neoumios ass & [LPm] $\infty$ 人 & (IPm) we & $c \angle \tau+N \nabla-1+z$ & 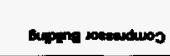 \\
\hline & & & & & minpara \\
\hline 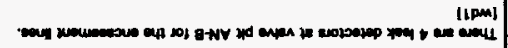 & $\mathbf{w}$ & [IPM] $\approx \alpha$ & [IPm] & 8AV & soun spower \\
\hline & & & & & 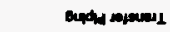 \\
\hline $\mathbf{w}$ & $\mathbf{m}$ & [LPm] $\bullet \mathrm{A}$ & ItPMI $\oplus \Lambda$ & VN & voppropep xwon. \\
\hline 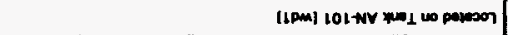 & $\mathbf{w}$ & 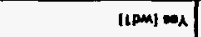 & [LPm| $\infty \mathbf{A}$ & 310 & 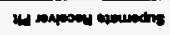 \\
\hline 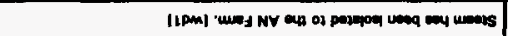 & $m$ & $\mathbf{v w}$ & on & $\mathbf{V N}$ & unens: \\
\hline $\mathbf{m}$ & $\mathbf{V N}$ & $\mathbf{m}$ & $\mathbf{V N}$ & $\mathbf{V N}$ & 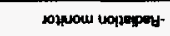 \\
\hline experedo peumentiv & $\mathbf{w}$ & $\infty$ & {$[z \|=\wedge$} & VN & N200\%- \\
\hline $\mathbf{w}$ & $\mathrm{v}$ & $\mathbf{W}$ & $\mathbf{V N}$ & $\mathrm{VN}$ & poump parp- \\
\hline $\mathbf{w}$ & VN & $\mathbf{m}$ & $\mathbf{V N}$ & $\mathrm{VN}$ & duns:- \\
\hline - upesedo pounnery & $w$ & $\infty$ & [Ot'OENYI $\neq \lambda$ & VN & بve. \\
\hline - Qquendo peumeor & $\mathbf{V N}$ & $\infty$ & [OENY] $\bullet A$ & VN & पTume entased $\mathrm{MG}$ - \\
\hline $\mathrm{n}$ & $\mathbf{w}$ & 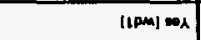 & {$\left[0 c^{\prime} E Z N Y\right][$ IPM] 00} & $\mathrm{VN}$ & woppoupep yoer- \\
\hline (1) & (1) & $6+\infty+\infty+\infty$ & 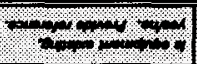 & $100+1 \%$ & 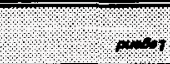 \\
\hline sxveurusos & vopupuos nenudintis & apanodo & Mesavd/aypenx马 & $\begin{array}{l}\text { mpuep } \\
\text { weudinb? nuojals }\end{array}$ & mozers \\
\hline
\end{tabular}

$0 \cdot \mathrm{net}$

100-3H-WM-as-JHM

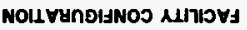

$\bullet \exists 78 \forall 1$ 


\begin{tabular}{|c|c|c|c|c|c|}
\hline$m$ & $m$ & $w$ & [IPM] on & vw & 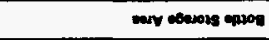 \\
\hline $\mathbf{n}$ & $\mathbf{m}$ & 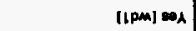 & 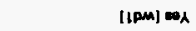 & $\mathbf{m}$ & mopens paneog sosow \\
\hline $\mathbf{m}$ & $\mathbf{w}$ & 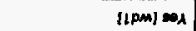 & $\mid(1 \mathrm{pm}) \mathrm{ma}$ & $\mathbf{w}$ & uopenoprs manows \\
\hline & & & & & 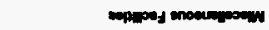 \\
\hline 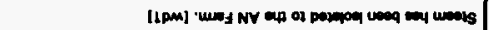 & $\mathbf{w}$ & $\mathbf{w}$ & or & VN & odent weops- \\
\hline & & & & & woms \\
\hline 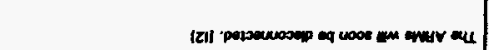 & $\mathbf{w}$ & 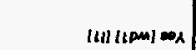 & 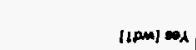 & $\mathbf{w}$ & 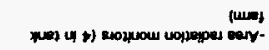 \\
\hline 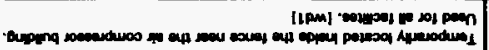 & $\mathbf{v N}$ & xneunuos ons & [LPM] & $\mathbf{v N}$ & sowoson- \\
\hline $\mathbf{V N}$ & $\mathbf{w}$ & 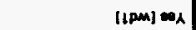 & 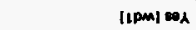 & $\mathbf{V N}$ & - 00011 ден- \\
\hline $\mathbf{n N}$ & $\mathbf{w}$ & 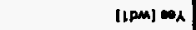 & [lama] $\infty \boldsymbol{\Lambda}$ & 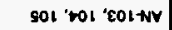 & Lownow AL- \\
\hline 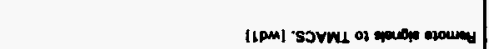 & vw & 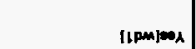 & 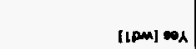 & YN & 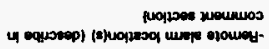 \\
\hline $\mathbf{w}$ & $\mathbf{V N}$ & {$[\because \mathrm{pm}] \operatorname{\infty os}$} & IIPma $\infty \infty_{\lambda}$ & $\mathbf{V N}$ & wro- \\
\hline 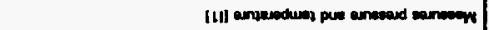 & $\mathbf{w}$ & ] & 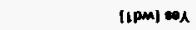 & VN & 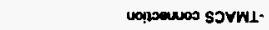 \\
\hline $\mathbf{W}$ & VN & llamp $\infty \lambda$ & {$[$ I $\mathrm{pm}]=0 \mathrm{\alpha}$} & $1 \angle Z+N Y-1+Z$ & apping vournear \\
\hline vN & VN & $\mathbf{W N}$ & ILPMI ON & VN & 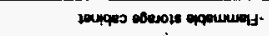 \\
\hline 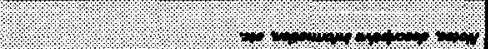 & $1+1+1$ & 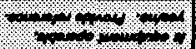 & 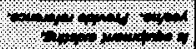 & 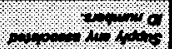 & 0.0 \\
\hline 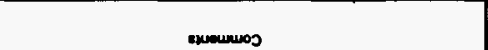 & volupeos wawdinb3 & 9qeedo & 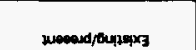 & 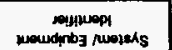 & wovers \\
\hline
\end{tabular}

0 คคด

LOO-ЭIH-WM- IS-รHM

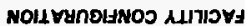

เ งาะท 1

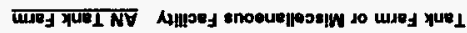


TABLE 4

FACILITY CONFIGURATION

WHC.SD-WM.HIE-OO7

Aov. 0

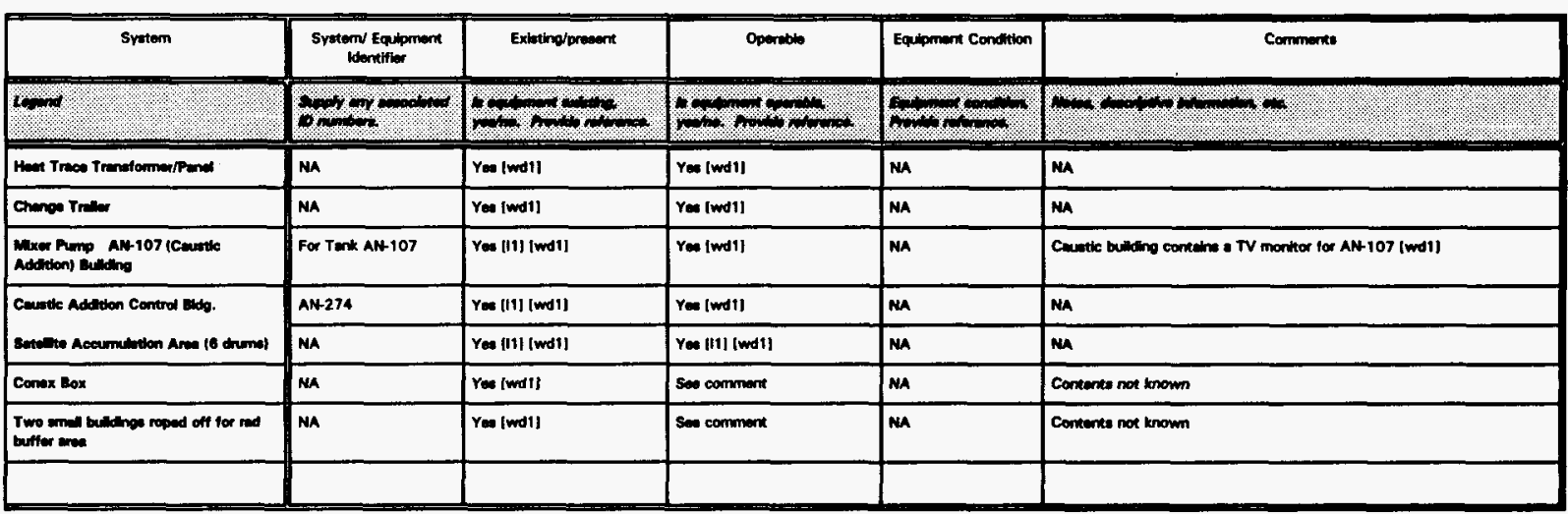




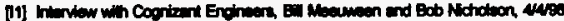

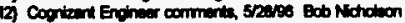

D1] Contion Angerment Sorver

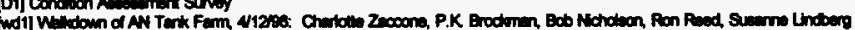

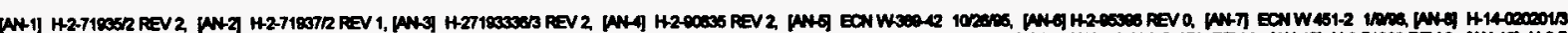

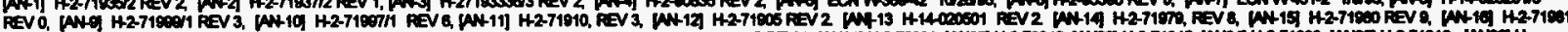

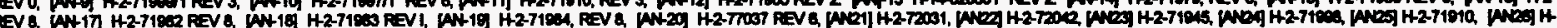

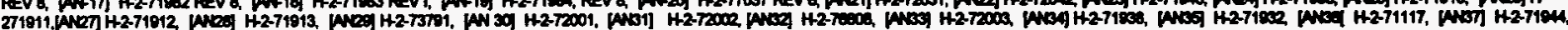

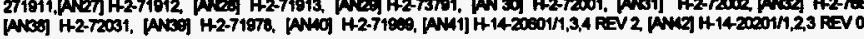




\begin{tabular}{|c|c|c|c|c|c|}
\hline System & System ID & $\begin{array}{c}\text { Radiatlon Source } \\
\geq 100 \mathrm{mRm}\end{array}$ & Protwetive Feature & Description of Protective Featurs & Commonte \\
\hline 10101, & $1+90^{\circ}$ & 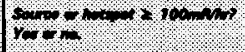 & $6 \%+1 \% \%$ & 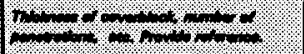 & $\%$ \\
\hline Tenke & $101-107$ & Yee & Sollorovel Cover & S I th thove terit dome crown [DII & Soll cover montioned for chindeling hoedinge \\
\hline Contrid pump pits & 01A-07A & Yos & Cover block & $\begin{array}{l}\text { 1'8" thick conc; Penwetrations: 1-42", 1- } \\
12^{\circ}, 1-4^{\prime \prime} \text { (AN28) (wd1) }\end{array}$ & 7 centred pump pits \\
\hline Annites porme phes & $018-078$ & Yen & Cover block & 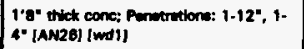 & 7 emile: pemp phe \\
\hline Lent dovection purve ghes & $01 c-07 c$ & Yes & Cover block & $\begin{array}{l}\text { 1'8* thick cone; Pencetrutione: 1-4", 1-8" } \\
\text { [AN26I Iwd1] }\end{array}$ & 7 loak detuction pits \\
\hline Ondin pit & 010,020 & Yes & Cover block & 1'ge thick conc: 2 pensetratione [AN29] & 2 dring phts \\
\hline Supeninto necelver ph & O1E & You & Cover block & $1^{\prime} 0^{\circ}$ thick conc; 1 penetrention [AN25] & 1 eppernate rocewive ph \\
\hline \multicolumn{6}{|l|}{ Other phe } \\
\hline Volvo $\mathbf{m}$ & ANAA & Ye: & Cow block & 1'E" thick conc; (AN3I) & I vive pk \\
\hline Volve Prt & AN-B & $Y=$ & Cover block & 1'e' theck conc; [AN31] & 1 valve pis \\
\hline Munh pit & 241.AN & Potentidily roe & Mutel plate & 14 on when matil cover [AN3O] & 1 them pin \\
\hline Senvioe ph & NA & No [wd1] & Grating Hoor & Open orutho [AN32] & Servica ph thelde thed with greted floor \\
\hline Cleen an box & AN $1-9$ & No & Motel phinto & 12 ge steot matil [AN33] & 9 coses \\
\hline Vent plus & AN $\$ 1,2,3,4$ & Yes & Cover block & 8* thich conc: (AN221 & 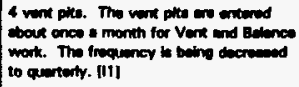 \\
\hline Denietew phe & TA-AN & Yos & Noter piate & $1.5^{\circ}$ eted platu cover [ANA] & 1 demivetion pit \\
\hline Putmery weel pot pht & For $\mathrm{X} 1$ syatem & Yes & Cover block & 6.5" thick concrote (ANB) & 1 and pot \\
\hline
\end{tabular}


TABLE 5

\begin{tabular}{|c|c|c|c|c|c|}
\hline Syatem & Syatem ID & $\begin{array}{l}\text { Reduntion Source } \\
\geq 100 \mathrm{~mA} / \mathrm{hr}\end{array}$ & Protective Feature & Description of Protective Foururs & Commente \\
\hline $6 \%$ & 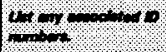 & 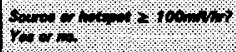 & 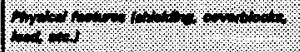 & 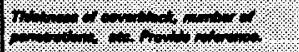 & $\%$ \\
\hline \multicolumn{6}{|l|}{ steem syrtem } \\
\hline -Steom traps & NA & NA & Ma & MA & There in no steam to the AN Fum. [woll] \\
\hline \multicolumn{6}{|l|}{ HVAC } \\
\hline $\begin{array}{l}\text { Tenk Vertiliation } \\
\text { Syateme }\end{array}$ & K1/K2 [wd1] & Yoe & K1 and K2 HEPA fiters and do-entrointers. & 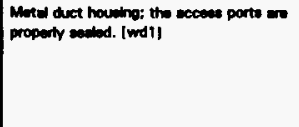 & 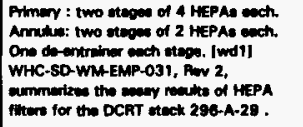 \\
\hline $\begin{array}{l}\text {-Other Vertllation } \\
\text { Systems }\end{array}$ & NA & NA & Ma & NA & MA \\
\hline Trenoter Lhee & NA & Ma & $\mathrm{Ma}$ & MA & $\sec$ Tats 7. \\
\hline Ory walle & NA & NA & MA & MA & 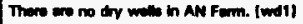 \\
\hline \multicolumn{6}{|l|}{ Devertinge } \\
\hline -Compreseor Bullitine & AN-273 & No [wd 1] & MA & Ma & 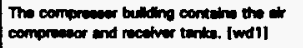 \\
\hline Inothumen Bulling & AN-271 & No [wdil] & Podiention montitor & CAM & m \\
\hline -Sorvice buliting & AN-273 & No [wd1] & Aadition montitor & CAM & MA \\
\hline -Chenos tralles & MO-820 & No [md1] & Redietion monitor & PCM1-8, HPT equlpment [wd1] & Chenpe triver controllod by HPTh. (wdil) \\
\hline Cavetic adetion control & AN-274 & No Indil] & MA & $\mu$ & 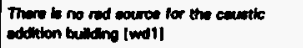 \\
\hline
\end{tabular}


TABLE 5

\begin{tabular}{|c|c|c|c|c|c|}
\hline Syotem & Syetem 10 & $\begin{array}{l}\text { Radiation Source } \\
\geq 100 \mathrm{~m} \text { (1) }\end{array}$ & Protective Feature & Description of Protecthe Feature & Commente \\
\hline $100,10,1$ & $1+10 \% \cdots+1$ & 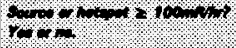 & $6+1 / 1 \%)$ & 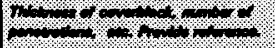 & +4 \\
\hline $\begin{array}{l}\text {-Two anned buildinge } \\
\text { moped off for rad buffer } \\
\text { erea. }\end{array}$ & Buildinge do not theve 10 . & Mo [wordl] & See comment & NA & Buldinge un controled by HPTE. [wd1] \\
\hline $\begin{array}{l}\text {-Sotente accummiletion } \\
\text { ares }\end{array}$ & No ID. & No [wd1] & Sen comment & NM & 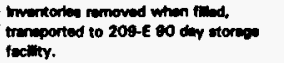 \\
\hline -Camera stetion & AN-103 & No [wd1] & Cover phe & $\begin{array}{l}\text { Ss cove plete; Puntretione: 2-6", } 1 \text { - } \\
10^{\circ}, 1-24^{*} \text { (wd1) }\end{array}$ & NA \\
\hline
\end{tabular}

[d1] OSD-T-151-00007. Row. H-17

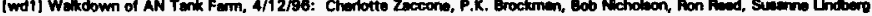

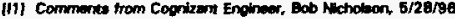

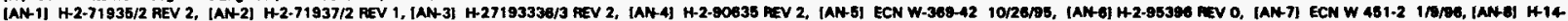

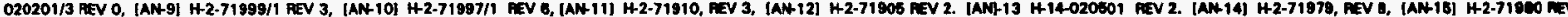

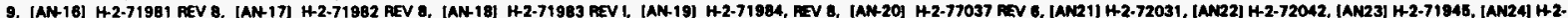

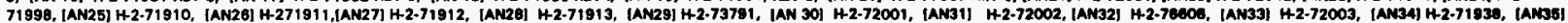
H-2-71932, [AN36! H-2-71117, [AN37] H-2.71944, [AN38] H-2-72031, (AN39] H-2-71978, (AN40] 71989 
TABLE 6

OSR/OSD SAFETY CONTROLS

\begin{tabular}{|c|c|c|c|c|c|c|}
\hline \multirow{2}{*}{\begin{tabular}{|l|} 
System/Structures/Components \\
Tanks AN 101-107
\end{tabular}} & \multicolumn{2}{|c|}{ OSR Limit } & \multirow{2}{*}{ OSD Limit } & Implementing Procedures & Verification & \multirow[t]{2}{*}{ Comments } \\
\hline & & & & NA & NA & \\
\hline Tank Waste Instrumentation & & & NA & NA & NA & \\
\hline Speclic gravily instr. system & & & NA & NA & NA & \\
\hline Temperabure probes (T.C Tree) & WHC-SD-WM-OSA-016 & 2.2 & NA & $\begin{array}{l}\text { TF-OR-A-03 } \\
\text { TF-OR-EF-AN } \\
\text { TF-OR-WST-01-D } \\
\text { TF-OR-WST-O1-W } \\
\text { TO-001-084 } \\
\text { TO-001-180 } \\
\text { TO-020-595 } \\
\text { TO-040-035 } \\
\text { TO-000-200 } \\
\text { TO-000-660 } \\
\text { TO-060-230 } \\
\text { TO-200-460 } \\
\text { TO-200-464 } \\
\text { TO-220-019 } \\
\text { TO-220-027 } \\
\text { TO-220-095 } \\
\text { TO-220-096 } \\
\text { TO-230-095 } \\
\text { TO-230-099 } \\
\text { TO-230-133 } \\
\text { TO-230-141 } \\
\text { TO-230-142 } \\
\text { TO-230-143 } \\
\text { TO-230-144 } \\
\text { TO-230-274 } \\
\text { TO-270-007 } \\
\text { TO-270-011 } \\
\text { TO-270-012 } \\
\text { TO-270-014 }\end{array}$ & FV DA & \\
\hline
\end{tabular}

05/31/96

NA $=$ Not applicable or not required 
TABLE 6

Tank Farm or Miscellaneous Facility AN

OSROSD SAFETY CONTROLS

\begin{tabular}{|c|c|c|c|c|c|}
\hline System/Structures/Components & OSR Limit & OSD Limit & Implementing Procedures & Verification & Comments \\
\hline & $\begin{array}{lll}\text { WHC-SD-WM-OSA-016 } & \text { SL } & 2.2 \\
\end{array}$ & NA & $\begin{array}{l}\text { TO-270-040 } \\
\text { TO-270-221 } \\
\text { TO-270-235 } \\
\text { TO-270-251 } \\
\text { TO-270-252 } \\
\text { TO-270-266 } \\
\text { TO-270-824 } \\
\text { TO-400-200 } \\
\text { TO-430-480 } \\
\text { TO-430-485 } \\
\text { TO-440-002 }\end{array}$ & $\overline{F V} \quad \overline{D A}$ & \\
\hline & WHC-SD-WM-OSA-016 LCO 3.2 .1 & $\overline{\text { NA }}$ & NA & FV & $\begin{array}{l}\text { LCO - Not Implemented by } \\
\text { ECN }\end{array}$ \\
\hline & WHC-SD-WM-OSR-016 SA 3.2 .2 .1 & NA & $\begin{array}{l}\text { TF-OR-A-03 } \\
\text { TF-OR-EF-AN } \\
\text { TF-OR-WST-01-D } \\
\text { TF-OR-WST-01-W } \\
\text { TO-001-184 } \\
\text { TO-040-660 } \\
\text { TO-220-095 } \\
\text { TO-220-096 } \\
\text { TO-230-095 } \\
\text { TO-230-099 } \\
\text { TO-230-133 } \\
\text { TO-230-141 } \\
\text { TO-230-142 } \\
\text { TO-230-143 } \\
\text { TO-230-144 } \\
\text { TO-270-007 } \\
\text { TO-270-011 } \\
\text { TO-270-012 } \\
\text { TO-270-035 } \\
\text { TO-270-040 } \\
\text { TO-400-200 } \\
\text { TO-430-480 } \\
\text { TO-430-485 } \\
\text { TO-440-002 }\end{array}$ & $\overline{F V} \quad$ DA & SP- Not Implemented by ECN \\
\hline
\end{tabular}

\section{5/31/96}

NA $=$ Not applicable or not required 
TABLE 6

Tank Farm or Miscellaneous Facility

AN

OSROSD SAFETY CONTROLS

\begin{tabular}{|c|c|c|c|c|c|}
\hline System/Structures/Components & OSR Limit & OSD Limit & Implementing Procedures & Verification & Comments \\
\hline 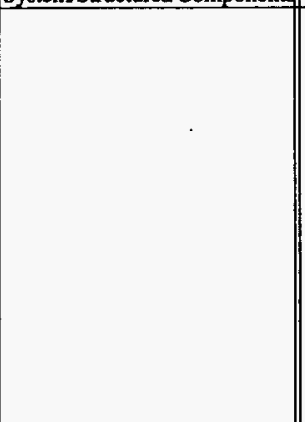 & NA & OSD-T-151-00007 7.2.6.a & $\begin{array}{l}\text { TO-001-182 } \\
\text { TO-001-184 } \\
\text { TO-040-760 } \\
\text { TO-200-010 } \\
\text { TO-220-095 } \\
\text { TO-230-095 } \\
\text { TO-230-130 } \\
\text { TO-230-274 } \\
\text { TO-270-040 } \\
\text { TO-270-204 } \\
\text { TO-270-221 } \\
\text { TO-270-236 } \\
\text { TO-270-252 } \\
\text { TO-270-266 } \\
\text { TO-270-826 } \\
\text { TO-270-828 } \\
\text { TO-400-200 } \\
\text { TO-430-480 } \\
\text { TO-600-080 }\end{array}$ & NA & \\
\hline
\end{tabular}

05/31/96

NA $=$ Not applicable or not required 
TABLE 6

Tank Farm or Miscellaneous Facility AN

OSR/OSD SAFETY CONTROLS

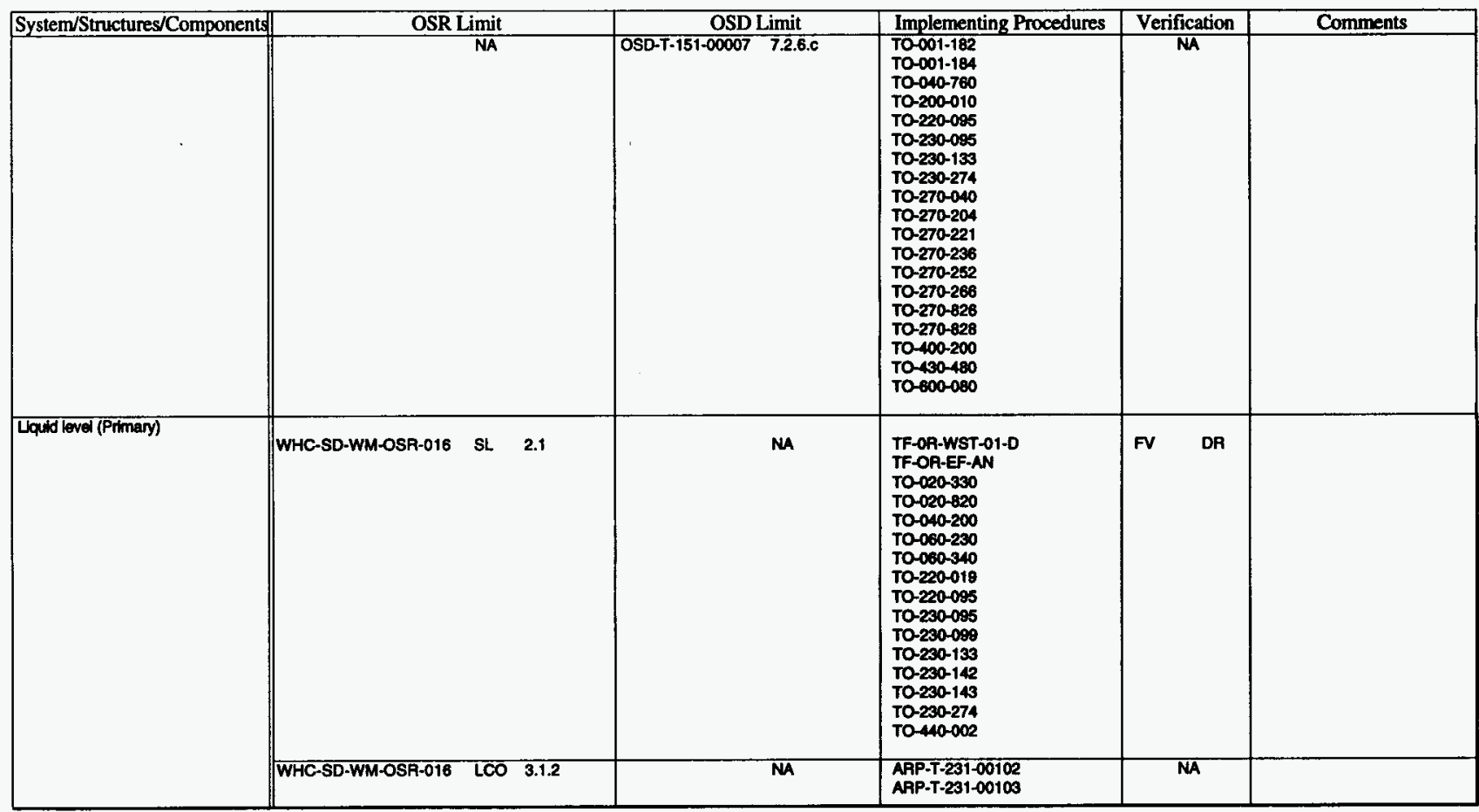

NA $=$ Not applicable or not required 
TABLE 6

Tank Farm or Miscellaneous Facility AN

OSR/OSD SAFETY CONTROLS

\begin{tabular}{|c|c|c|c|c|c|}
\hline System/Structures/Components & OSR Limit & OSD Limit & Implementing Procedures & Verification & Comments \\
\hline 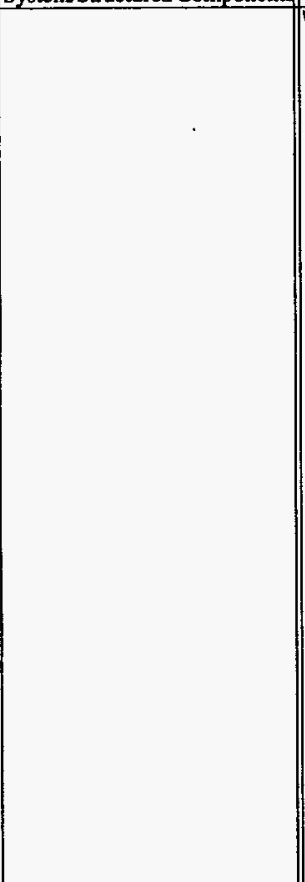 & WHC-SD-WM-OSR-016 LCO 3.1 .2 & NA & $\begin{array}{l}\text { ARP-T-231-00105 } \\
\text { ARP-T-231-00106 } \\
\text { ARP-T-271-00101 } \\
\text { ARP-T-271-00102 } \\
\text { ARP-T-271-00103 } \\
\text { ARP-T-271-00104 } \\
\text { ARP-T-271-00105 } \\
\text { ARP-T-271-00106 } \\
\text { ARP-T-271-00107 } \\
\text { ARP-T-271-00106 } \\
\text { TF-OR-A-03 } \\
\text { TF-OR-EF-AN } \\
\text { TF-OR-WST-01-D } \\
\text { TO-001-084 } \\
\text { TO-001-180 } \\
\text { TO-001-184 } \\
\text { TO-020-025 } \\
\text { TO-020-330 } \\
\text { TO-020-595 } \\
\text { TO-020-620 } \\
\text { TO-040-200 } \\
\text { TO-080-105 } \\
\text { TO-060-230 } \\
\text { TO-060-340 } \\
\text { TO-200-460 } \\
\text { TO-220-027 } \\
\text { TO-220-095 } \\
\text { TO-220-096 } \\
\text { TO-230-095 } \\
\text { TO-230-099 } \\
\text { TO-230-133 } \\
\text { TO-230-142 } \\
\text { TO-230-149 } \\
\text { TO-230-144 } \\
\text { TO-230-274 } \\
\text { TO-270-007 } \\
\text { TO-270-012 } \\
\text { TO-270-014 } \\
\text { TO-270-235 } \\
\text { TO-270-251 }\end{array}$ & NA & \\
\hline
\end{tabular}


WHC-SD-WM-HIE-007

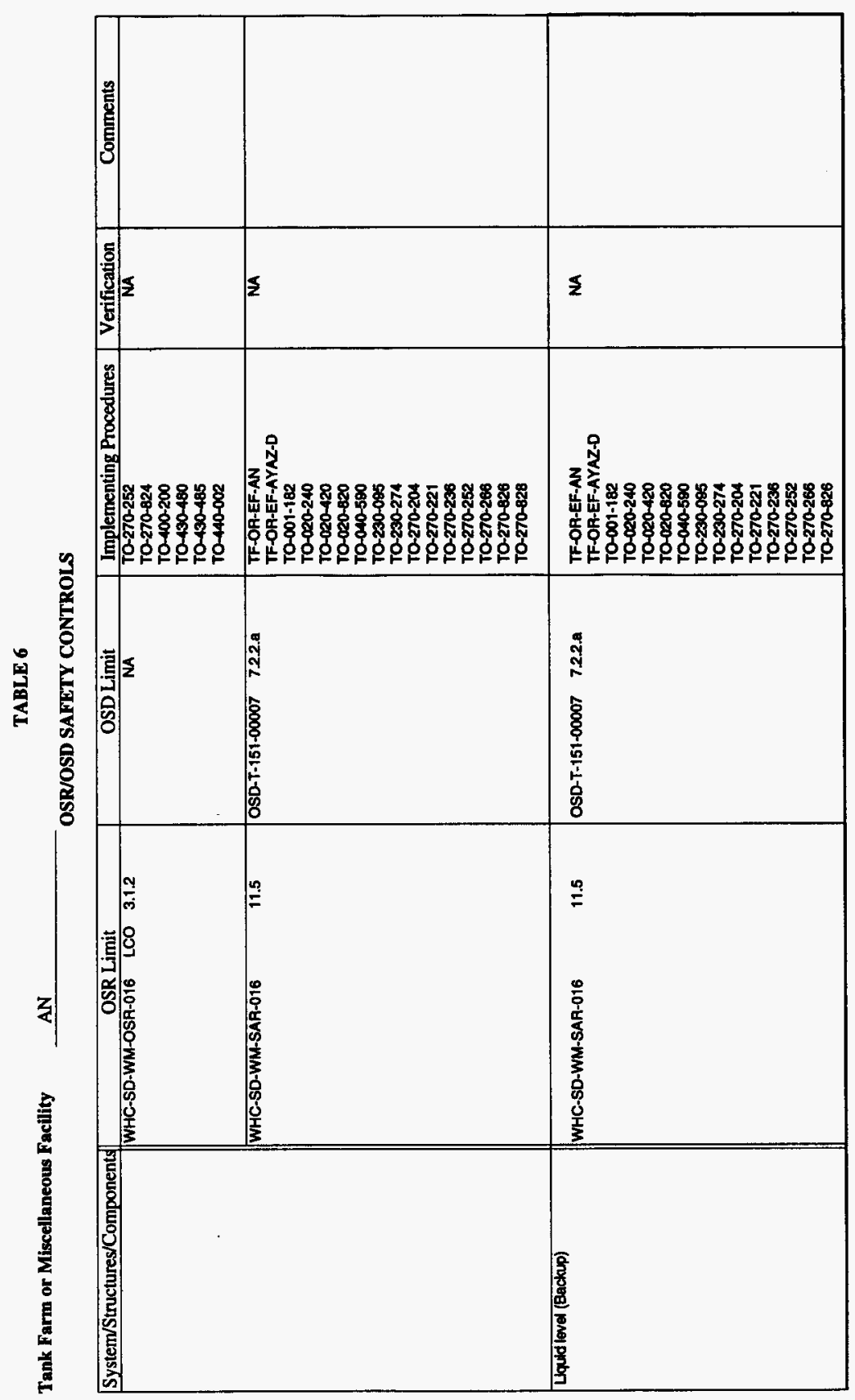

REV 0

$\underset{⿴ 囗 十}{Y}$

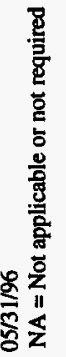


TABLE 6

Tank Farm or Miscellaneous Facility

AN

OSROSD SAFETY CONTROLS

\begin{tabular}{|c|c|c|c|c|c|c|c|}
\hline \multirow[t]{2}{*}{ System/Structures/Components } & \multicolumn{2}{|c|}{ OSR Limit } & \multirow{2}{*}{\multicolumn{2}{|c|}{$\begin{array}{l}\text { Implementing Procedures } \\
\text { T0-270-828 }\end{array}$}} & \multirow{2}{*}{\multicolumn{2}{|c|}{$\frac{\text { Verification }}{N A}$}} & \multirow[t]{2}{*}{ Comments } \\
\hline & WHC-SD-WM-SAR-016 & & & & & & \\
\hline Hitgl level probe & \multicolumn{2}{|r|}{ NA } & & MA & \multicolumn{2}{|c|}{ NA } & \\
\hline \multirow[t]{2}{*}{$\begin{array}{l}\text { Tank primary vapor space pressure } \\
\text { morthor }\end{array}$} & WHC-SD-WM-OSA-016 & SL 2 & $\begin{array}{l}\text { TF-FT-239-001 } \\
\text { TF-FT-279-001 } \\
\text { TF-OR-WST-01-D } \\
\text { TO-040-035 } \\
\text { TO-060-101 } \\
\text { TO-060-230 } \\
\text { TO-060-340 } \\
\text { TO-230-095 } \\
\text { TO-230-133 } \\
\text { TO-230-142 }\end{array}$ & & FV & DR & \\
\hline & WHC-SD-WM-OSA-016 & LCOS & $\begin{array}{l}\text { ARP-T-231-00102 } \\
\text { ARP-T-231-00103 } \\
\text { ARP-T-231-00104 } \\
\text { ARP-T-231-00105 } \\
\text { ARP-T-231-00106 } \\
\text { ARP-T-271-00101 } \\
\text { ARP-T-271-00102 } \\
\text { ARP-T-271-00104 } \\
\text { ARP-T-271-00105 } \\
\text { ARP-T-271-00106 } \\
\text { ARP-T-271-00107 } \\
\text { ARP-T-271-00108 } \\
\text { TF-FT-239-001 } \\
\text { TF-FT-279-001 } \\
\text { TO-001-084 } \\
\text { TO-001-184 } \\
\text { TO-040-035 } \\
\text { TO-060-101 } \\
\text { TO-060-230 } \\
\text { TO-060-340 } \\
\text { TO-200-019 } \\
\text { TO-200-464 }\end{array}$ & & FV & $\overline{D A}$ & \\
\hline
\end{tabular}

05/31/96

NA $=$ Not applicable or not required 
TABLE 6

Tank Farm or Miscellaneous Facility AN

OSROSD SAFETY CONTROLS

\begin{tabular}{|c|c|c|c|c|c|}
\hline System/Structures/Components & OSR Limit & OSD Limit & Implementing Procedures & Verification & Comments \\
\hline 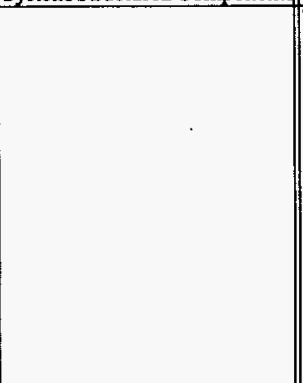 & WHC-SD-WM-OSR-016 LCO 3.3 .1 & NA & $\begin{array}{l}\text { TO-220-027 } \\
\text { TO-220-095 } \\
\text { TO-230-095 } \\
\text { TO-230-099 } \\
\text { TO-230-133 } \\
\text { TO-230-141 } \\
\text { TO-230-142 } \\
\text { TO-230-143 } \\
\text { TO-230-144 } \\
\text { TO-230-274 } \\
\text { TO-270-007 } \\
\text { TO-270-011 } \\
\text { TO-270-012 } \\
\text { TO-270-235 } \\
\text { TO-270-824 } \\
\text { TO-400-200 } \\
\text { TO-430-480 }\end{array}$ & $\overline{F V}$ DA & \\
\hline & $\begin{array}{llll}\text { WHC-SD-WM-OSP-016 } & \text { LCO } & 3.3 .2 \\
\end{array}$ & NA & $\begin{array}{l}\text { APP-T-231-00101 } \\
\text { ARP-T-231-00105 } \\
\text { ARP-T-231-00106 } \\
\text { ARP-T-271-00102 } \\
\text { ARP-T-271-00103 } \\
\text { ARP-T-271-00104 } \\
\text { ARP-T-271-00105 } \\
\text { ARP-T-271-00106 } \\
\text { ARP-T-271-00107 } \\
\text { ARP-T-271-00108 } \\
\text { ARP-T-601-00295 } \\
\text { TF-OR-A-03 } \\
\text { TF-OR-EF-AN } \\
\text { TF-OR-WST-01-D } \\
\text { TO-040-035 } \\
\text { TO-060-101 } \\
\text { TO-060-230 } \\
\text { TO-060-340 } \\
\text { TO-230-095 } \\
\text { TO-230-133 } \\
\text { TO-230-142 }\end{array}$ & FV DF & \\
\hline
\end{tabular}

$05 / 31 / 96$

NA $=$ Not applicable or not required 
TABLE 6

\begin{tabular}{|c|c|c|c|c|c|c|}
\hline \multirow[t]{3}{*}{ System/Structures/Components } & \multicolumn{2}{|c|}{ OSR Limi! } & \multirow{2}{*}{$\frac{\text { OSD Limit }}{\text { NA }}$} & \multirow[b]{2}{*}{$\begin{array}{l}\text { Implementing Procedures } \\
\text { ARP-T-231-00101 } \\
\text { TF-OR-A-03 } \\
\text { TF-OR-EF-AN } \\
\text { TF-OR-WST-01-D } \\
\text { TO-040-035 } \\
\text { TO-060-101 } \\
\text { TO-060-340 }\end{array}$} & \multirow{2}{*}{$\begin{array}{l}\text { Verification } \\
\text { FV DR }\end{array}$} & \multirow[t]{2}{*}{ Comments } \\
\hline & WHC-SD-WM-OSR-016 & $\begin{array}{ll}\text { SA } & \text { 3.3.2.1 } \\
\end{array}$ & & & & \\
\hline & WHC-SD-WM-SAR-016 & 11.6 & OSD-T-151-00007 7.2 .5 & $\begin{array}{l}\text { TO-060-105 } \\
\text { TO-080-341 } \\
\text { TO-400-245 }\end{array}$ & NA & \\
\hline Gas monltoring/SHMS & & NA & NA & NA & NA & \\
\hline Mass spectrometer & & NA & NA & MA & NA & \\
\hline TMACS & & NA & NA & NA & NA & \\
\hline Mullithnction instrument tree (MIT) & & NA & NA & MA & NA & \\
\hline TV Cameras & & NA & NA & NA & NA & \\
\hline Vepor sampler & & NA & MA & NA & NA & \\
\hline Studge level thacator & & NA & NA & MA & MA & \\
\hline $\begin{array}{l}\text { Tank Annulus } \\
\text { Instrumentabion }\end{array}$ & & NA & NA & MA & MA & \\
\hline Leak detection manual tape & & NA & NA & NA & NA & \\
\hline
\end{tabular}

\section{$05 / 31 / 96$}

NA $=$ Not applicable or not required 
TABLE 6

Tank Farm or Miscellaneous Facility

AN

OSR/OSD SAFETY CONTROLS

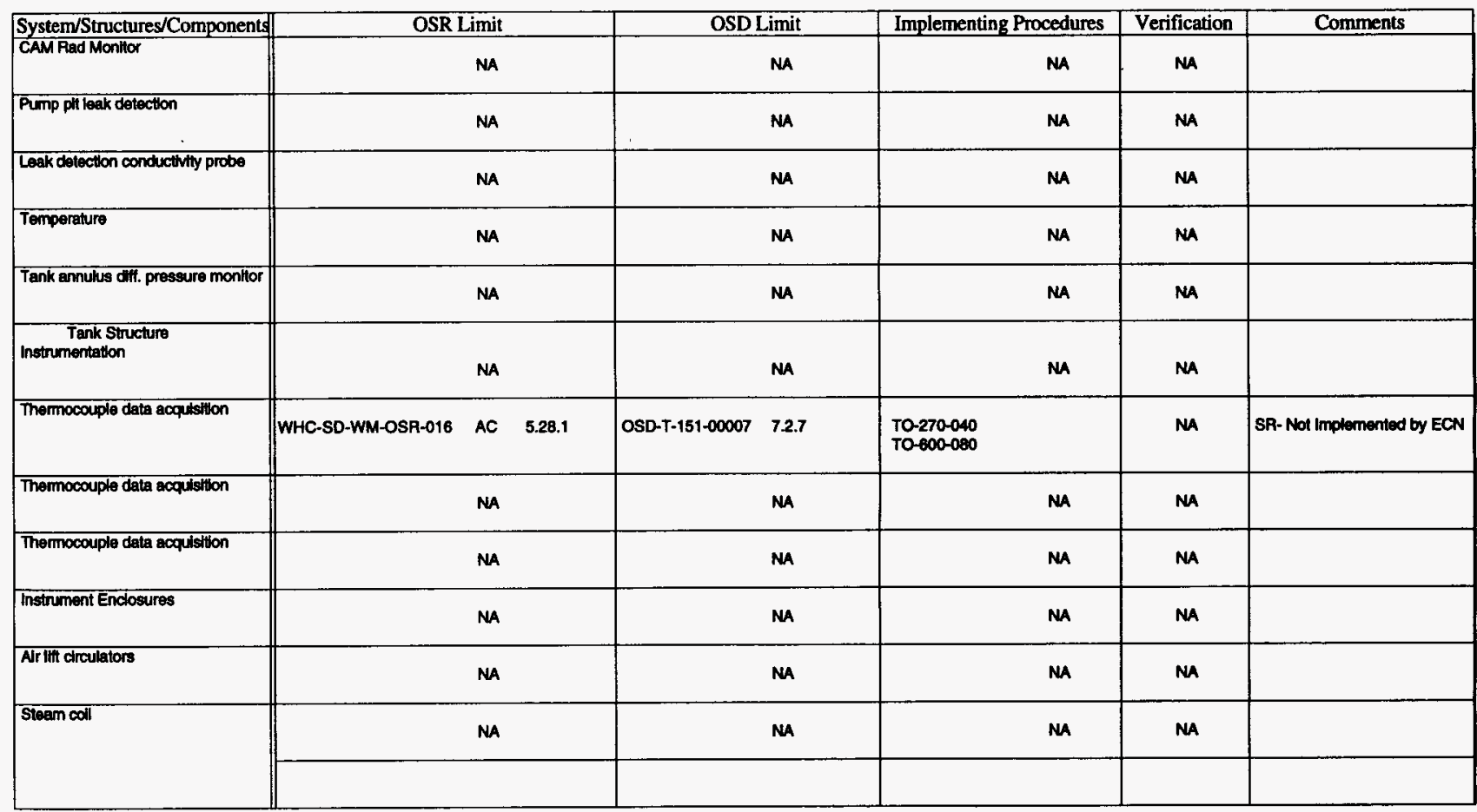

$05 / 31 / 96$

NA $=$ Not applicable or not required 
TABLE 6

Tank Farm or Miscellaneous Facility AN

OSR/OSD SAFETY CONTROLS

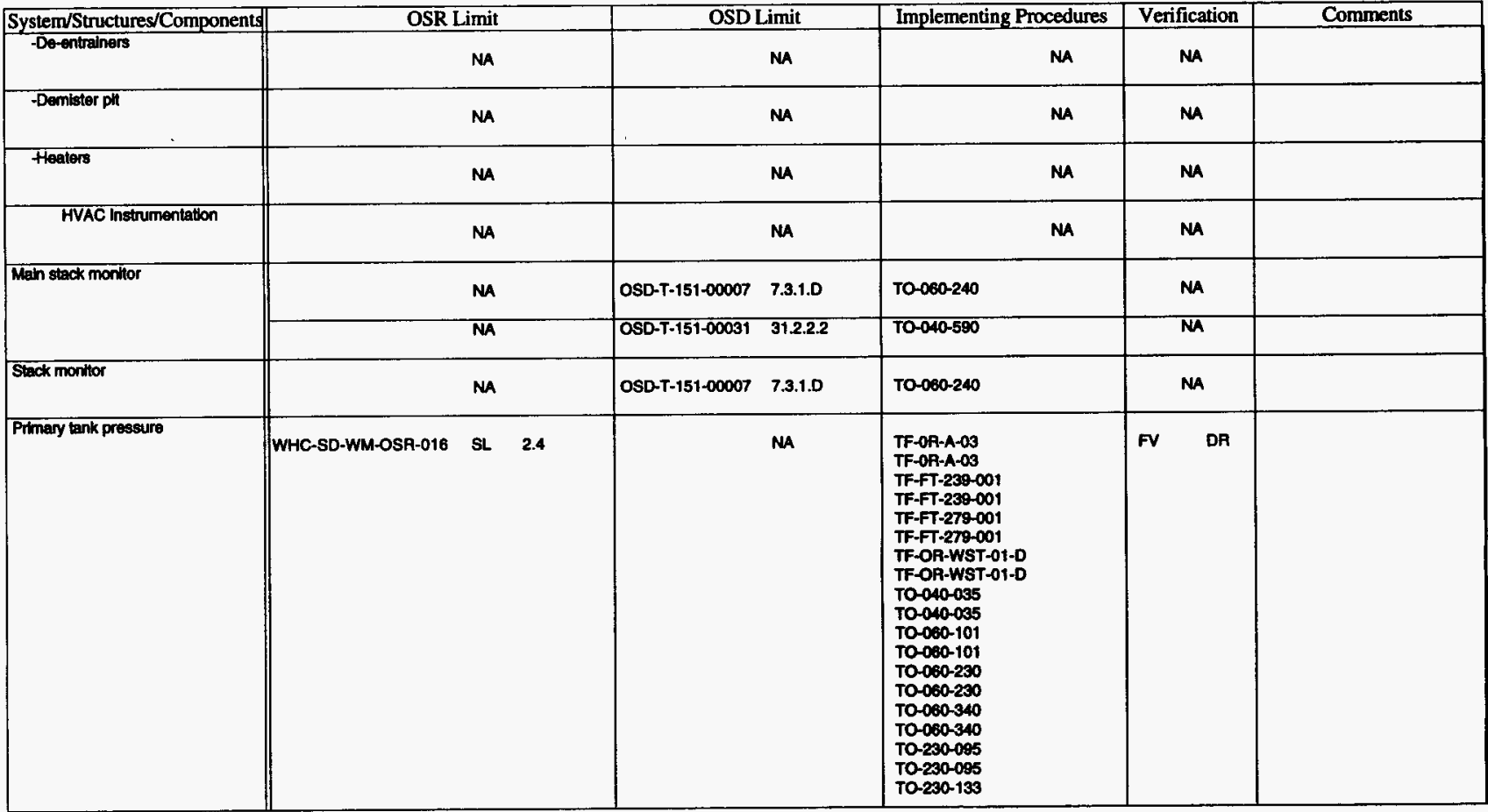

05/31/96

NA $=$ Not applicable or not required 
TABLE 6

Tank Farm or Miscellaneous Facility AN

\section{OSR/OSD SAFETY CONTROLS}

\begin{tabular}{|c|c|c|c|c|c|c|c|}
\hline \multirow[t]{2}{*}{ System/Structures/Components } & \multicolumn{2}{|c|}{ OSR Limit } & \multirow{2}{*}{\multicolumn{2}{|c|}{$\frac{\text { OSD Limit }}{\text { NA }}$}} & \multirow{2}{*}{$\begin{array}{l}\text { Implementing Procedures } \\
\text { TO-230-133 } \\
\text { TO-230-142 } \\
\text { TO-230-142 }\end{array}$} & $\frac{\text { Verification }}{\text { FV DA }}$ & \multirow[t]{2}{*}{ Comments } \\
\hline & WHC-SD-WM-OSR-016 & $\mathrm{SL} \quad 2.4$ & & & & FV $\quad$ DA & \\
\hline - & WHC-SD-WM-OSR-016 & LCO 3.4 .1 & OSD-T-151-00007 & 7.3.1.A & $\begin{array}{l}\text { TO-060-101 } \\
\text { TO-060-104 } \\
\text { TO-060-105 } \\
\text { TO-060-230 } \\
\text { T0-060-240 } \\
\text { TO-060-340 } \\
\text { TO-060-341 }\end{array}$ & NA & $\begin{array}{l}\text { LCO-Not Implemented by } \\
\text { ECN }\end{array}$ \\
\hline & & NA & OSD-T-151-00007 & 7.3.1.8 & $\begin{array}{l}\text { TO-060-105 } \\
\text { TO-060-230 } \\
\text { TO-060-240 } \\
\text { TO-060-340 } \\
\text { TO-060-341 }\end{array}$ & NA & \\
\hline \begin{tabular}{|l|l|} 
Difierentlal pressure indicators \\
\end{tabular} & & NA & & NA & NA & MA & \\
\hline Temperature indicators & & NA & & NA & NA & NA & \\
\hline Stack flow moler & & NA & & NA & NA & NA & \\
\hline Pirmary Seal Pot & & NA & & NA & NA & NA & \\
\hline Level element & & NA & & NA & NA & NA & \\
\hline Draln & & NA & & MA & NA & Na & \\
\hline Heat trace & & NA & & MA & Ma & $\mathbf{M A}$ & \\
\hline
\end{tabular}

05/31/96

NA $=$ Not applicable or not required 
Tank Farm or Miscellaneous Facility

AN

\begin{tabular}{|c|c|c|c|c|c|}
\hline System/Structures/Components & OSR Limit & OSD Limit & Implementing Procedures & Verification & Comments \\
\hline Annulus Sad Pof Plt & NA & NA & NA & MA & \\
\hline Level element & NA & NA & NA & NA & \\
\hline Drain & NA & NA & NA & MA & \\
\hline Ventllation Pth & NA & NA & NA & MA & \\
\hline Ventilation PHt 11 & NA & NA & NA & MA & \\
\hline Annulus duct pressure & MA & NA & NA & NA & \\
\hline CAM & NA & NA & NA & NA & \\
\hline Flow control valve & NA & NA & NA & $\mathrm{MA}$ & \\
\hline Ventlation Pit 12 & NA & NA & MA & M & \\
\hline Arnulus duct pressure & NA & NA & NA & NA & \\
\hline CAM & NA & NA & MA & NA & \\
\hline Fow control valve & NA & NA & NA & NA & \\
\hline Ventitation PAt w3 & NA & M & M & NA & \\
\hline & & & & & \\
\hline
\end{tabular}

\section{$05 / 31 / 96$}

NA = Not applicable or not required

OSR/OSD SAFETY CONTROLS

TABLE 6 
WHC-SD-WM-HIE-007 REV 0

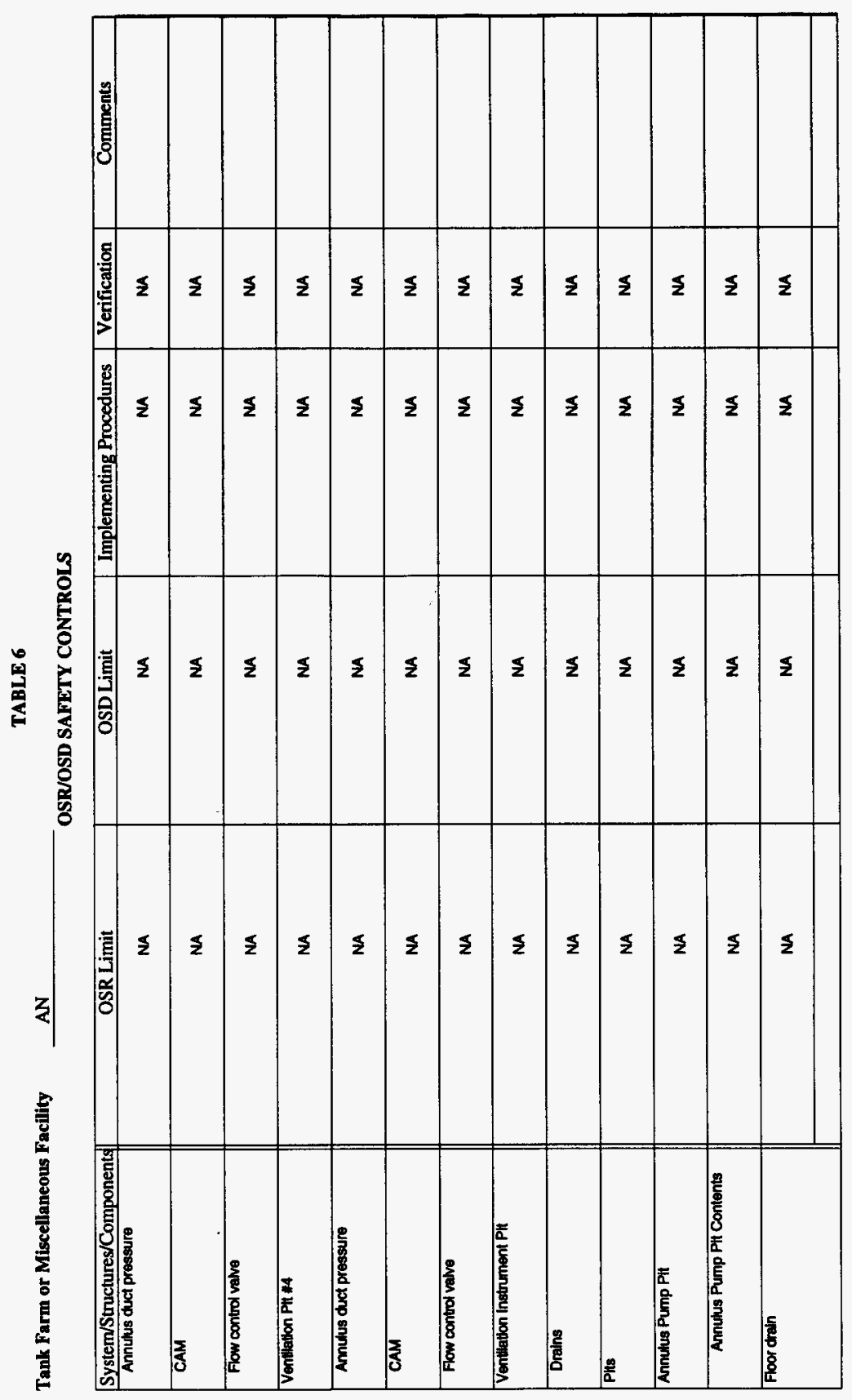

n)

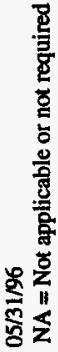


TABLE 6

Tank Farm or Miscellaneous Facility

AN

OSR/OSD SAFETY CONTROLS

\begin{tabular}{|c|c|c|c|c|c|}
\hline System/Structures/Components & OSR Limit & OSD Limit & Implementing Procedures & Verification & Comments \\
\hline Sump & NA & NA & NA & NA & \\
\hline Laak datection & NA & NA & MA & NA & \\
\hline Pilser spray ing & NA & NA & MA & NA & \\
\hline Central Pump Plt & NA & NA & NA & NA & \\
\hline Contral Pump Pit Contents & NA & NA & MA & NA & \\
\hline Leak delection & NA & NA & NA & NA & \\
\hline Floor drain & NA & NA & NA & NA & \\
\hline Check Valve & NA & NA & MA & NA & \\
\hline Portable pit spray (1" AW & NA & NA & MA & NA & \\
\hline Dlstributor PIt & NA & NA & NA & NA & \\
\hline Distrioutor Plt Contents & NA & NA & MA & NA & \\
\hline Stulce Plt & NA & NA & NA & MA & \\
\hline Stuice PHt Contents & NA & MA & MA & Ma & \\
\hline
\end{tabular}

05/31/96

NA $=$ Not applicable or not required 
TABLE 6

Tank Farm or Miscellaneous Facility

AN

OSR/OSD SAFETY CONTROLS

\begin{tabular}{|c|c|c|c|c|c|}
\hline System/Structures/Components & OSR Limit & OSD Limit & Implementing Procedures & Verification & Comments \\
\hline Heel PIt & NA & NA & NA & $\mathbf{M A}$ & \\
\hline Heel Pit Conlents & NA & NA & NA & NA & \\
\hline Leak detection pump pht & NA & NA & NA & NA & \\
\hline $\begin{array}{l}\text { Laak Delection PH/Sump } \\
\text { instrumentalion }\end{array}$ & NA & NA & NA & NA & \\
\hline $\begin{array}{l}\text { Level sensing system (SpG-WF } \\
\text { sump) }\end{array}$ & NA & OSD-T-151-00007 7.2.2.b & $\begin{array}{l}\text { TF-OR-EF-AN } \\
\text { TO-020-240 } \\
\text { TO-020-420 } \\
\text { TO-020-820 } \\
\text { TO-040-580 } \\
\text { TO-230-095 } \\
\text { TO-270-204 } \\
\text { TO-270-221 } \\
\text { TO-270-236 } \\
\text { TO-270-252 } \\
\text { TO-270-265 } \\
\text { TO-270-826 } \\
\text { TO-270-828 }\end{array}$ & NA & $\begin{array}{l}\text { SpG-WF on 241-AN-103 is } \\
\text { plugged; a zip cord ts used } \\
\text { instead }\end{array}$ \\
\hline Padiation monitor (sump) & NA & NA & MA & NA & \\
\hline Leak detection system (pit) & NA & NA & NA & MA & \\
\hline Temperature & NA & NA & NA & NA & \\
\hline
\end{tabular}

\section{$05 / 31 / 96$}

NA $=$ Not applicable or not required 
TABLE 6

Tank Farm or Miscellaneous Facility

AN

OSR/OSD SAFETY CONTROLS

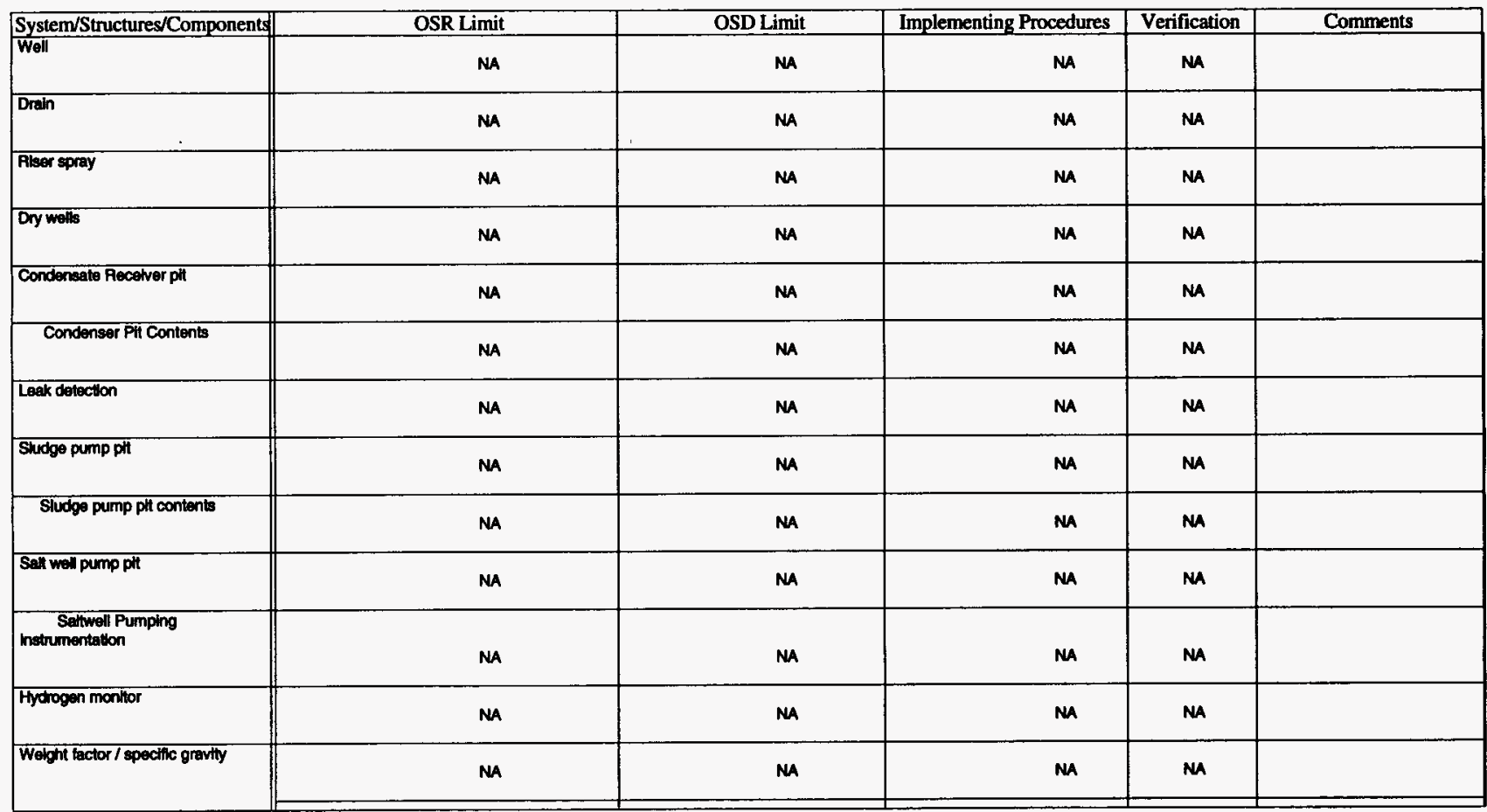

$05 / 31 / 96$

NA = Not applicable or not required 
TABLE 6

Tank Farm or Miscellaneous Facility

AN

OSR/OSD SAFETY CONTROLS

\begin{tabular}{|c|c|c|c|c|c|}
\hline System/Structures/Components & OSR Limit & OSD Limit & Implementing Procedures & Verification & Comments \\
\hline Drain pit & NA & NA & Ma & MM & \\
\hline Drain Pll Contents & NA & NA & NA & NA & \\
\hline Drain & NA & NA & NA & NA & \\
\hline Loak dotoction & NA & NA & NA & NA & \\
\hline \begin{tabular}{|l} 
Portable ph spray \\
\end{tabular} & NA & MA & NA & MA & \\
\hline $\begin{array}{l}\text { Valve PH/ Transfor PH/ D Diversion } \\
\text { Boxes (2) }\end{array}$ & NA & NA & NA & NA & \\
\hline Ptt or Diversion Box Contents & NA & NA & NA & NA & \\
\hline Leak detection & NA & NA & NA & NA & \\
\hline Valve postion indication & NA & NA & M & NA & \\
\hline Pipeline hlow monltoring & NA & NA & NA & NA & \\
\hline Drain & NA & NA & NA & NA & \\
\hline Steam & NA & NA & NA & NA & \\
\hline Pressure switches & NA & Ma & NA & NA & \\
\hline
\end{tabular}

05/31/96

$\mathrm{NA}=$ Not applicable or not required 
WHC-SD-WM-HIE-007 REV 0

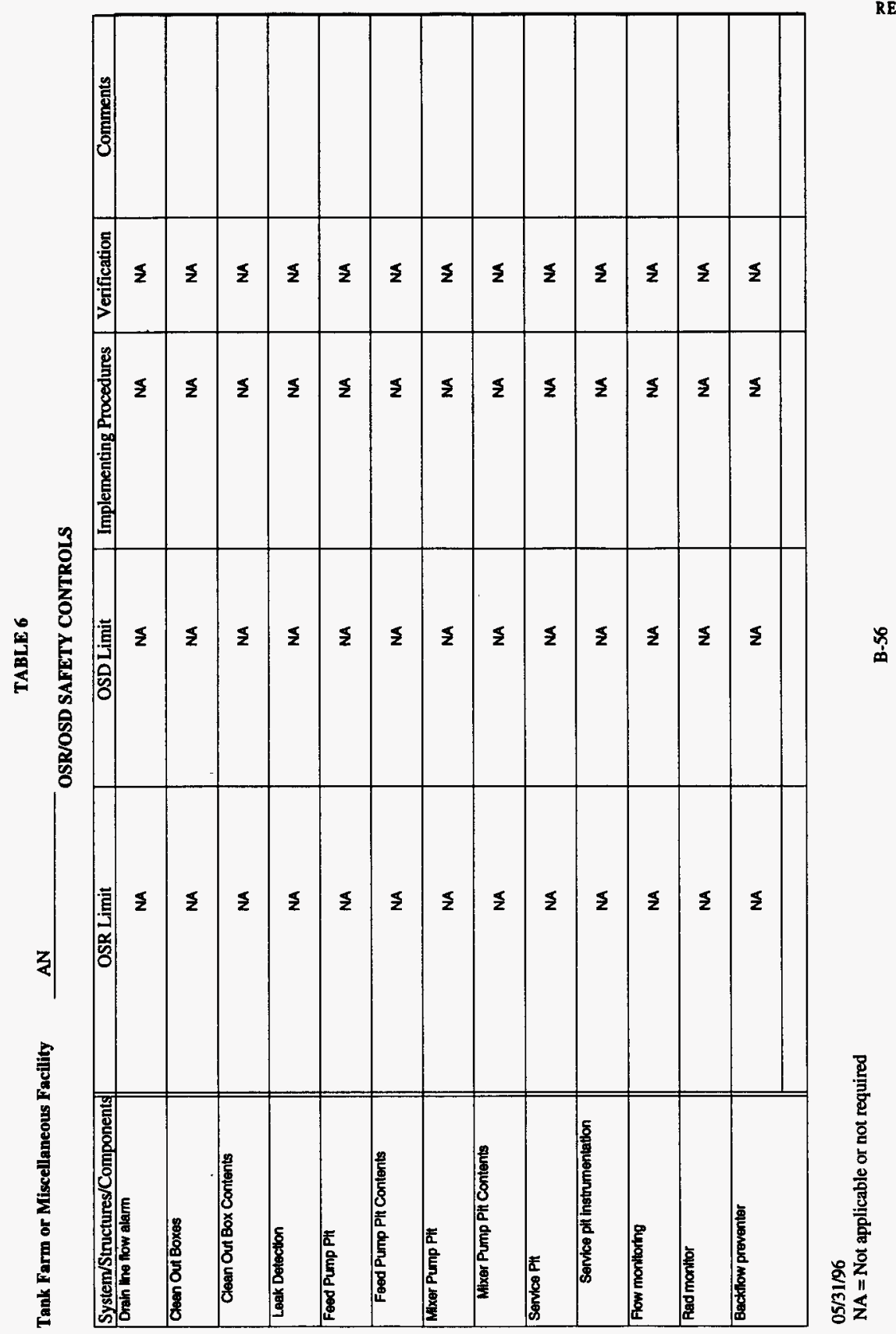


TABLE 6

Tank Farm or Miscellaneous Facility

AN

OSR/OSD SAFETY CONTROLS

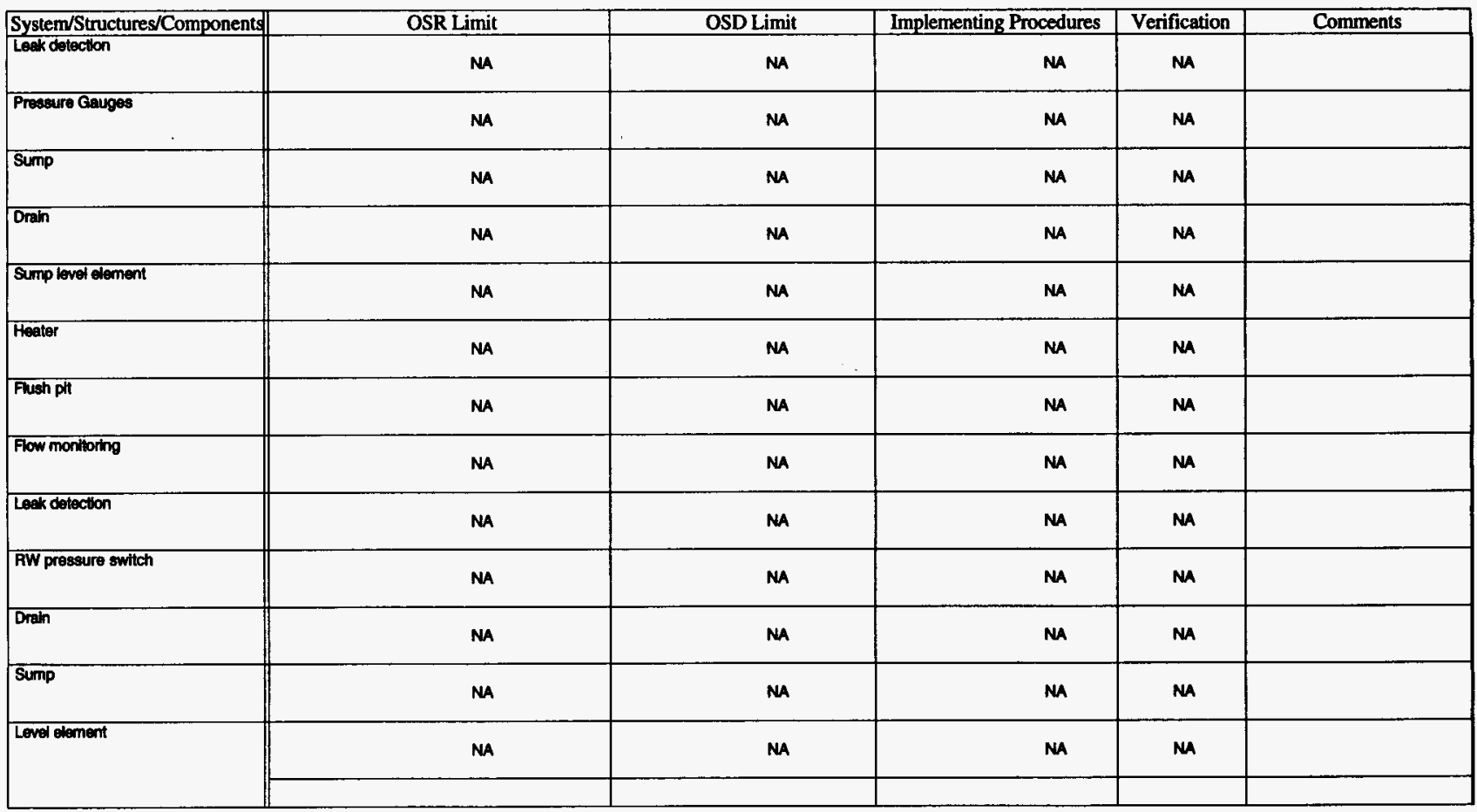

05/31/96

NA $=$ Not applicable or not required 
WHC-SD-WM-HIE-007 REV 0

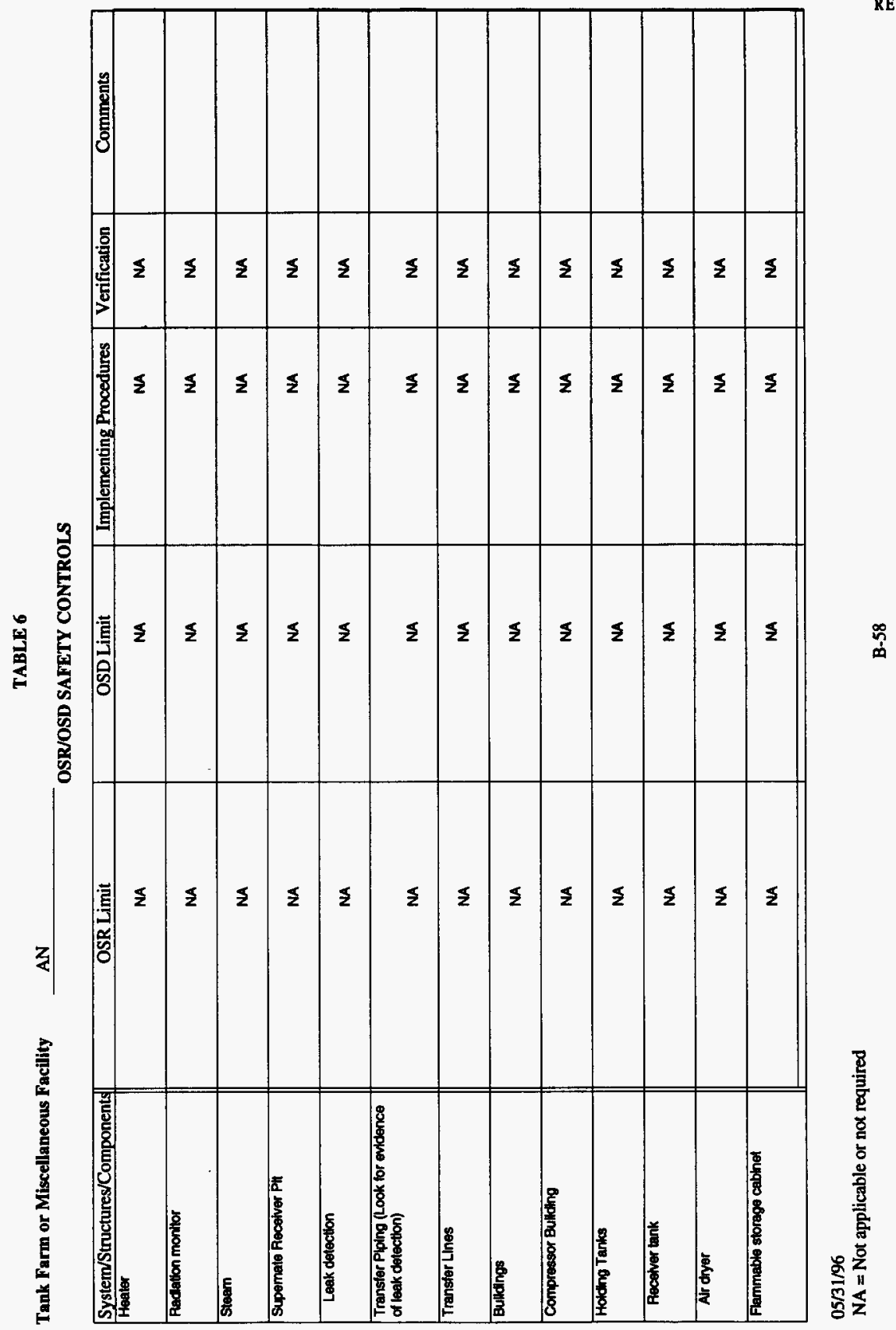


TABLE 6

\begin{tabular}{|c|c|c|c|c|c|}
\hline System/Structures/Components & OSR Limit & OSD Limit & Implementing Procedures & Verification & Comments \\
\hline Instrument Buliding & NA & NA & NA & NA & \\
\hline TMACS connection & NA & Ma & Ma & NA & \\
\hline CAM & NA & NA & NA & NA & \\
\hline $\begin{array}{l}\text { Remole alarm location(s) (describe } \\
\text { in comment section) }\end{array}$ & NA & NA & NA & NA & \\
\hline TV montior & NA & NA & NA & NA & \\
\hline Miscollaneous instrumentation & NA & NA & NA & NA & \\
\hline Heat trace & NA & NA & NA & NA & \\
\hline Viscometer & NA & NA & NA & NA & \\
\hline 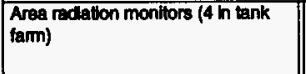 & NA & NA & NA & NA & \\
\hline Stern & NA & NA & MA & NA & \\
\hline Strain traps & NA & NA & NA & NA & \\
\hline Miscollaneous Faciltiles & NA & NA & NA & M & \\
\hline
\end{tabular}

$05 / 31 / 96$

$\mathrm{NA}=\mathrm{Not}$ applicable or not required 
TABLE 6

\begin{tabular}{|c|c|c|c|c|c|}
\hline System/Structures/Components & OSR Limit & OSD Limit & Implementing Procedures & Verification & Comments \\
\hline Electrical Substation & NA & NA & Na & NA & \\
\hline Motor Control Station & NA & NA & Ma & NA & \\
\hline Botile Storage Area & NA & NA & Ma & NA & \\
\hline Heat Trace Transtormer/Panel & NA & NA & NA & NA & \\
\hline Change Traller & NA & NA & NA & NA & \\
\hline $\begin{array}{l}\text { Mixer Pump AN-107 (Caustlc } \\
\text { Addtilon) Buldding }\end{array}$ & NA & NA & NA & NA & \\
\hline Caustic Addition Skdd & NA & NA & NA & NA & \\
\hline $\begin{array}{l}\text { Satellite Accumulation Arsa (6) } \\
\text { drums) }\end{array}$ & NA & NA & NA & NA & \\
\hline Conex Box & NA & NA & NA & NA & \\
\hline $\begin{array}{l}\text { Two smail bulldings roped off for } \\
\text { rad buffer area }\end{array}$ & NA & NA & NA & NA & \\
\hline
\end{tabular}

05/31/96

$\mathrm{NA}=$ Not applicable or not required 


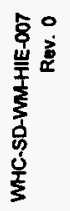

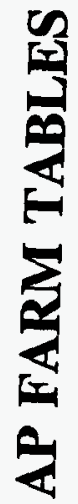

B-61 
WHC-SD-WML-HIE-007

Rev. 0

TWRS FEAR MATRIX TABLE VERIFICATION REVIEW APPROVALS May 31, 1996

The following information has been assembled. organized, and documented in the attached TWRS Final Safety Analysis Report (FSAR) Matrix Tables in support of Tank Farms FSAR development. Science Applications International Corporation (SAIC), along with the assistance of ICF Kaiser Hanford and the Westinghouse Hanford Company, assembled this data from controlled drawings and databases, supporting documents, as well as other site documentation. Where possible, the data has been field verified by performing field walkdowns of the particular facility of interest. The data also have been reviewed for its completeness and accuracy by the TWRS Facility Operations Design Authority and the Cognizant Engineer assigned to the respective facility. The information, to the best of the knowledge and the time allotted by the undersigned, is true. accurate. and complete as permitted by the referenced documents and drawings shown on the respective tables. No efforts were attempted or made by the authors and/or reviewers to substantiate the reference information shown on the tables beyond the stated qualifiers as noted on the individual facility matrix tables.

AP Tank Farm

Tank Farm or Facility

Preprecknos

PK Brockman

SAIC Lead
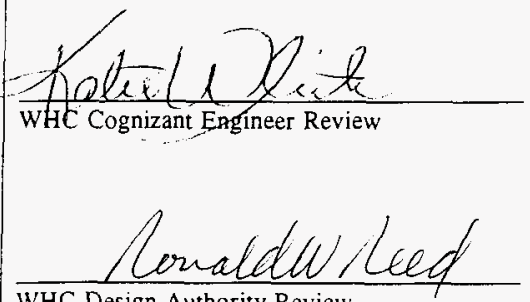

WHC Design Authority Review
$5 / 31 k 16$

Date
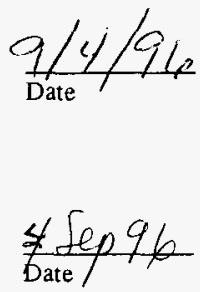

$B-62$ 


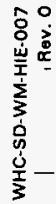
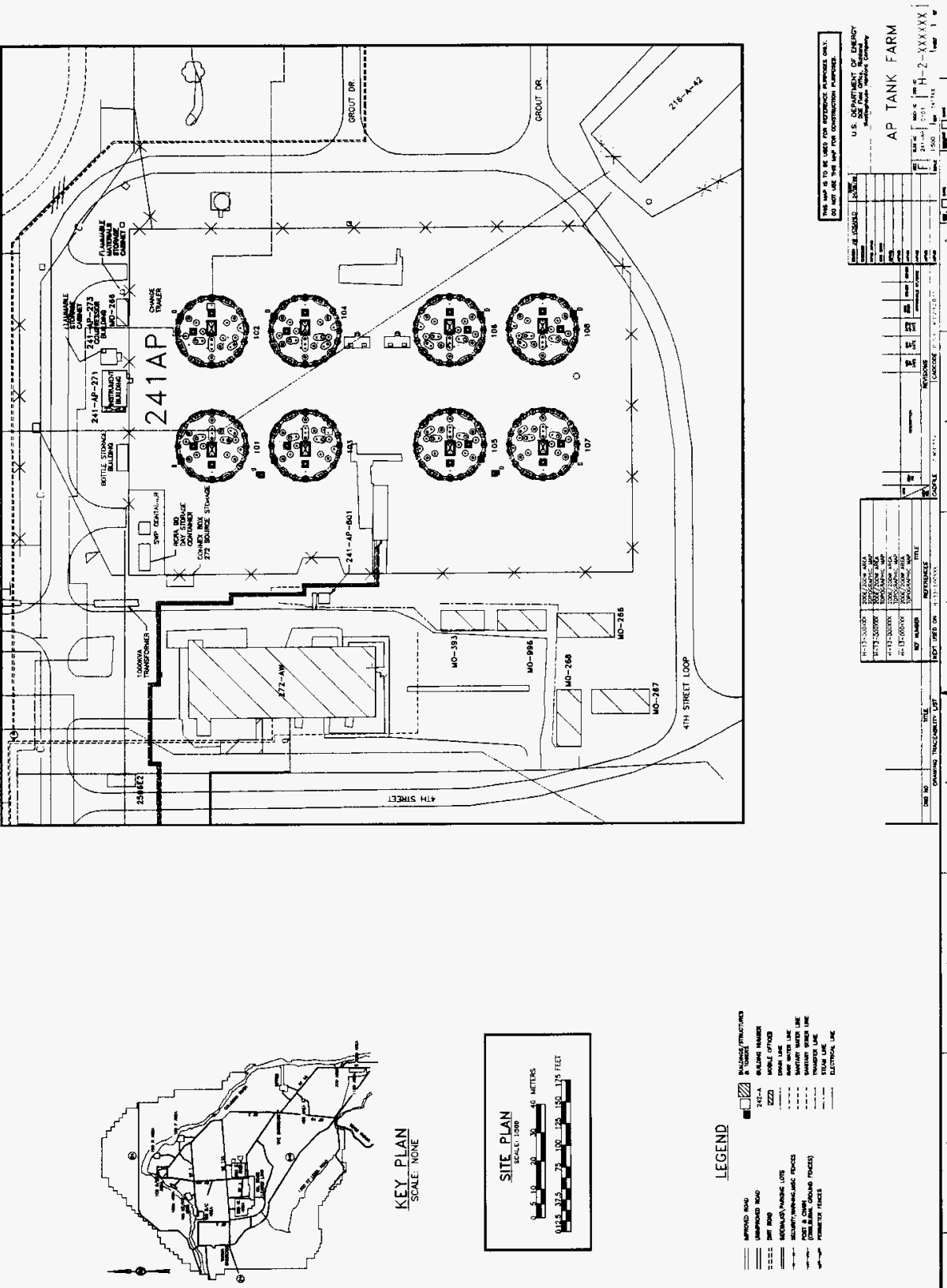
$\$ 98$

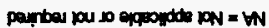

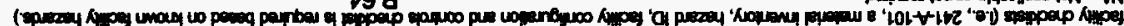

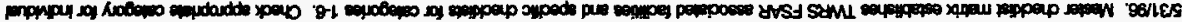

\begin{tabular}{|c|c|c|c|c|c|c|c|c|}
\hline 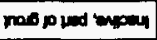 & $x$ & $x$ & $x$ & $w$ & $x$ & $\mathbf{W}$ & pad dund exper & QWO-ALITE \\
\hline 7000 pou $=00$ & $w$ & $w$ & $w$ & $w$ & $w$ & $w$ & ad dund pees & a+o-d \\
\hline$w$ & $x$ & $x$ & $\bar{x}$ & $w$ & $x$ & $x$ & yd dind pees & $0-20-\alpha+1+2$ \\
\hline$w$ & $x$ & $x$ & $x$ & $w$ & $x$ & $x$ & vad dund poss & verdatitz \\
\hline$w$ & $x$ & $x$ & $x$ & $w$ & $x$ & $x$ & pd expes & cuz-dvine \\
\hline$w$ & $x$ & $x$ & $x$ & $w$ & $x$ & $x$ & 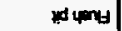 & avite \\
\hline d 4 us $8: 900$ or & WN & W & $\mathbf{w}$ & $w$ & W & $w$ & $x 00$ no weop & $\pm 10-\Delta v 800$ \\
\hline ov $4: 90000$ & $w$ & $w$ & W & $w$ & $w$ & $w$ & xoq no weop & $320-8+900$ \\
\hline or us 89000 ou & $\mathbf{w}$ & $w$ & w & $m$ & $w$ & $w$ & $x 09$ no & OLO-NVEOS \\
\hline rovlte we was & W & $w$ & $w$ & w & $w$ & W & tod axph & $\Delta v i t z$ \\
\hline$w$ & $x$ & $x$ & $x$ & $x$ & $x$ & $x$ & 150 & $001-a+1+5$ \\
\hline $\mathbf{w}$ & $x$ & $x$ & $x$ & $x$ & $x$ & $x$ & 150 & $\angle 01-d v i k z$ \\
\hline$W$ & $x$ & $x$ & $x$ & $x$ & $x$ & $x$ & 150 & Dol-atithe \\
\hline$w$ & $x$ & $x$ & $x$ & $x$ & $x$ & $x$ & 150 & Sol-d $\forall-162$ \\
\hline$w$ & $x$ & $x$ & $x$ & $x$ & $x$ & $x$ & 150 & not-dtive \\
\hline$w$ & $x$ & $x$ & $x$ & $x$ & $x$ & $x$ & 150 & $\infty 01-d v i t z$ \\
\hline $\mathbf{w}$ & $x$ & $x$ & $x$ & $x$ & $x$ & $x$ & 150 & $201-d \forall 162$ \\
\hline$w$ & $x$ & $x$ & $x$ & $x$ & $x$ & $x$ & 150 & $10 t-d A-1 B Z$ \\
\hline 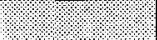 & (1) & 4. & . & (2) & 6. & 4.4 & 1. & wowat \\
\hline פסטרמן & 20 & d & 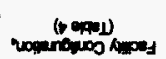 & (Exply) & 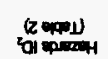 & 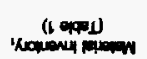 & $\mathbf{d} \mathbf{k}_{\perp}$ & S \\
\hline
\end{tabular}

0 ำ변

LOO-JIH-WM-OS-JHM

\section{ISTODEHO YQ15W}

Maty dy 
AP FARM

WHC-SO-WM-HIE-007

MASTER CHECAST

\begin{tabular}{|c|c|c|c|c|c|c|c|c|}
\hline Faciny & Type & $\begin{array}{l}\text { Materied Mneribory, } \\
\text { (Table 1) }\end{array}$ & Hande $1 D_{2}$ & $\begin{array}{l}\text { Urique Phenomens, } \\
\text { (Twbis 3) }\end{array}$ & 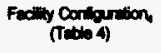 & Pad loves & Contrate & Comment \\
\hline 241.AP-OTE & Mes pamp pt & M & $x$ & $M$ & $x$ & $x$ & $x$ & hactive pot of orou \\
\hline 241-AP-OTF & Meer pump pit & NA & $x$ & $M$ & $x$ & $x$ & $x$ & madive put of oran \\
\hline 241-AP-271 & $\begin{array}{l}\text { Inothrmant } \\
\text { buiding }\end{array}$ & MA & $x$ & $M$ & $x$ & $x$ & $x$ & M \\
\hline 241-NP-701 & $\begin{array}{l}\text { Comprosor } \\
\text { builing }\end{array}$ & $x$ & $x$ & MA & $x$ & $x$ & $x$ & M \\
\hline 241AP-273 & Senvioe building & MA & M & M & M & $m$ & M & 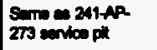 \\
\hline \multirow[t]{3}{*}{ 241APA } & Vhope ph & $x$ & $x$ & M & $x$ & $x$ & $x$ & $m$ \\
\hline & socumite & $x$ & $x$ & M & $x$ & $x$ & $x$ & MA \\
\hline & Crempo railer & $x$ & $x$ & $M$ & $x$ & $\mathbf{x}$ & $x$ & M \\
\hline
\end{tabular}




\begin{tabular}{|c|c|c|c|c|c|c|c|c|}
\hline \multirow{2}{*}{ 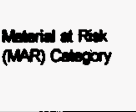 } & \multirow{2}{*}{ Capecity } & \multicolumn{5}{|c|}{ Muried theritory } & \multirow{2}{*}{ aminy } & \multirow{2}{*}{ Commonts } \\
\hline & & amailication & Typo & $\begin{array}{l}\text { Phypican } \\
\text { Form }\end{array}$ & Volume o a Actily & Trentiont & & \\
\hline $10.010 .0 \%$ & $\frac{1}{10}$ & $\frac{10}{4+1}$ & $x_{\infty}^{\infty}$ & 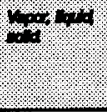 & \% & $\frac{\theta+1}{1+1}$ & $\frac{1}{1}+1 \%$ & $\%=$ \\
\hline \multicolumn{9}{|l|}{ 1. Thrk Corterts } \\
\hline 241AP-101 & $1.16 \mathrm{Mbol}(0)$ & DSSF (a) & apemos (a) & Lavid (b) & $735 \mathrm{Kagl}$ (c) & No & $m$ & $\mathbf{M A}$ \\
\hline 241AP-102 & $1.16 \mathrm{Mbol}(\mathrm{a})$ & CP (a) & apernino (a) & Maid (b) & $1000 \mathrm{Kod}(c)$ & No & $M$ & $m$ \\
\hline 241-AP-103 & $1.18 \mathrm{Mbel}(\mathrm{a})$ & DN (a) & apernato (0) & Inquid (D) & $25 \mathrm{~kg}(\mathrm{al}$ & No & MA & $m$ \\
\hline 241AP-104 & $1.18 \mathrm{Noll}(0)$ & ON (a) & upenosos (a) & Viavid (b) & $1123 \mathrm{Kad}(\mathrm{Ag})$ & No & $m$ & m \\
\hline 241AP-105 & $9.16 \mathrm{MgOad}(\mathrm{al})$ & DSSF (a) & (1) & $\operatorname{cosid(1)}$ & $155 \mathrm{Kogl}(c)$ & No & $m$ & $m$ \\
\hline 241AP-108 & $1.16 \mathrm{Nom}$ (n) & DN (a) & apendes (n) & Iapid (b) & $107 \mathrm{Kad}(\mathrm{CA})$ & No & $m$ & $m$ \\
\hline 241-AP-107 & $1.18 \mathrm{Mpod}$ (a) & DN (a) & apernolo (l) & Vand (b) & $25 \mathrm{~kg}(\mathrm{da})$ & No & M & $m$ \\
\hline 241-AP-106 & $1.16 \mathrm{Nad}(\mathrm{a})$ & DN (0) & spermen (0) & aqud (b) & $20 \mathrm{Kol} \Leftrightarrow$ & No & $\mathbf{m}$ & $M$ \\
\hline 2 Tark Hendranow & & & & & & & & \\
\hline
\end{tabular}




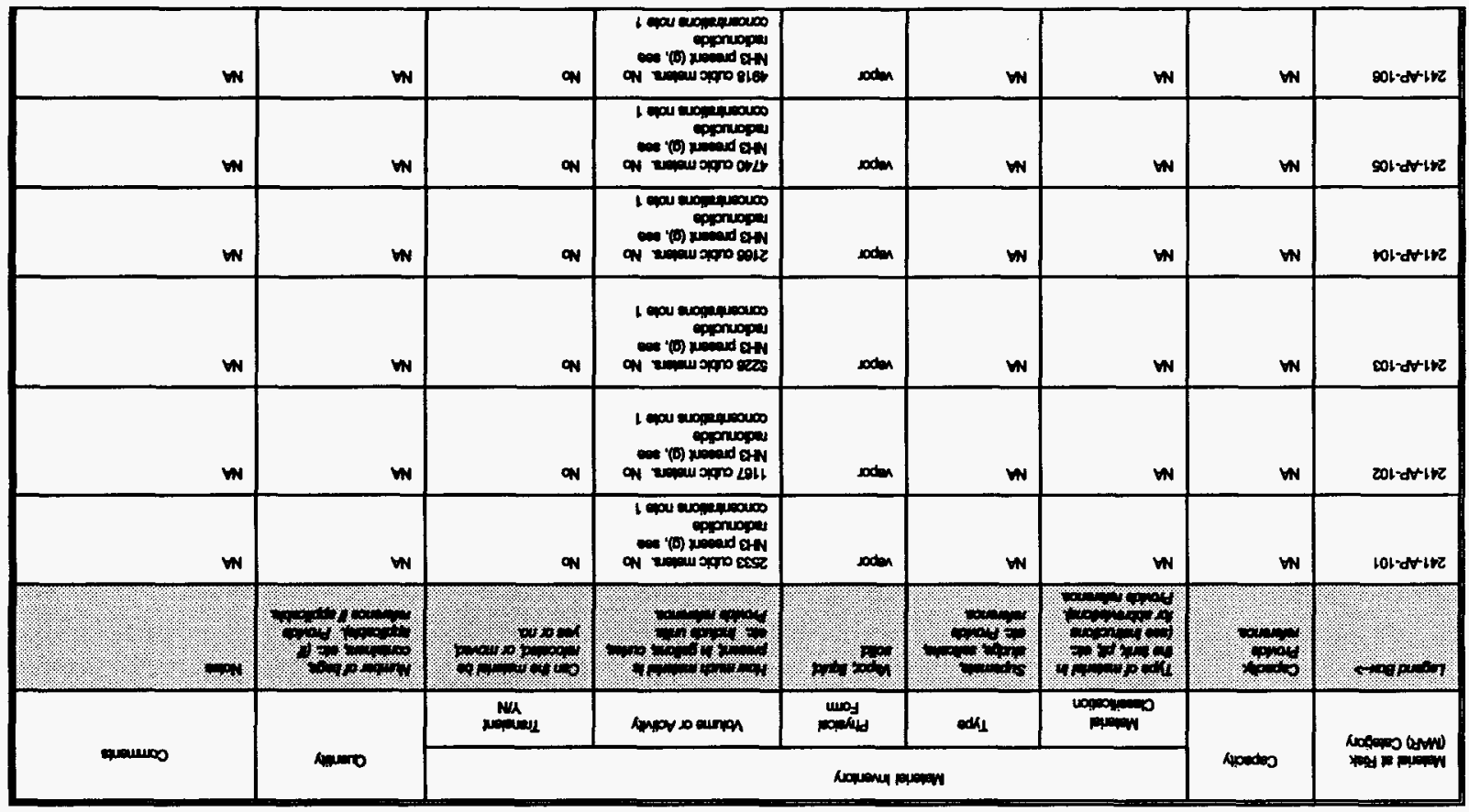

0 noy

100-3HH.WM as -OHM

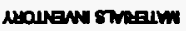

I ITEI 


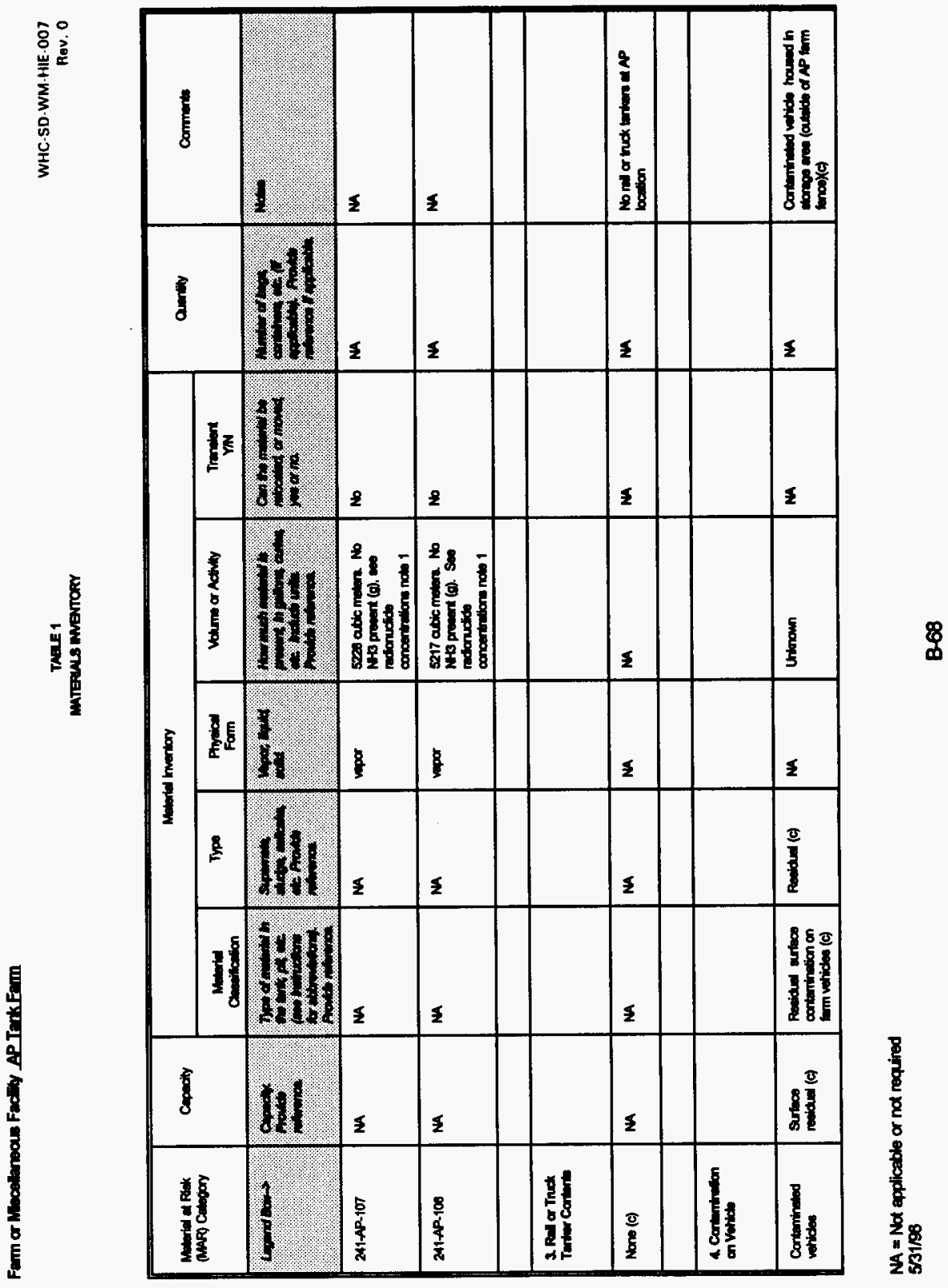




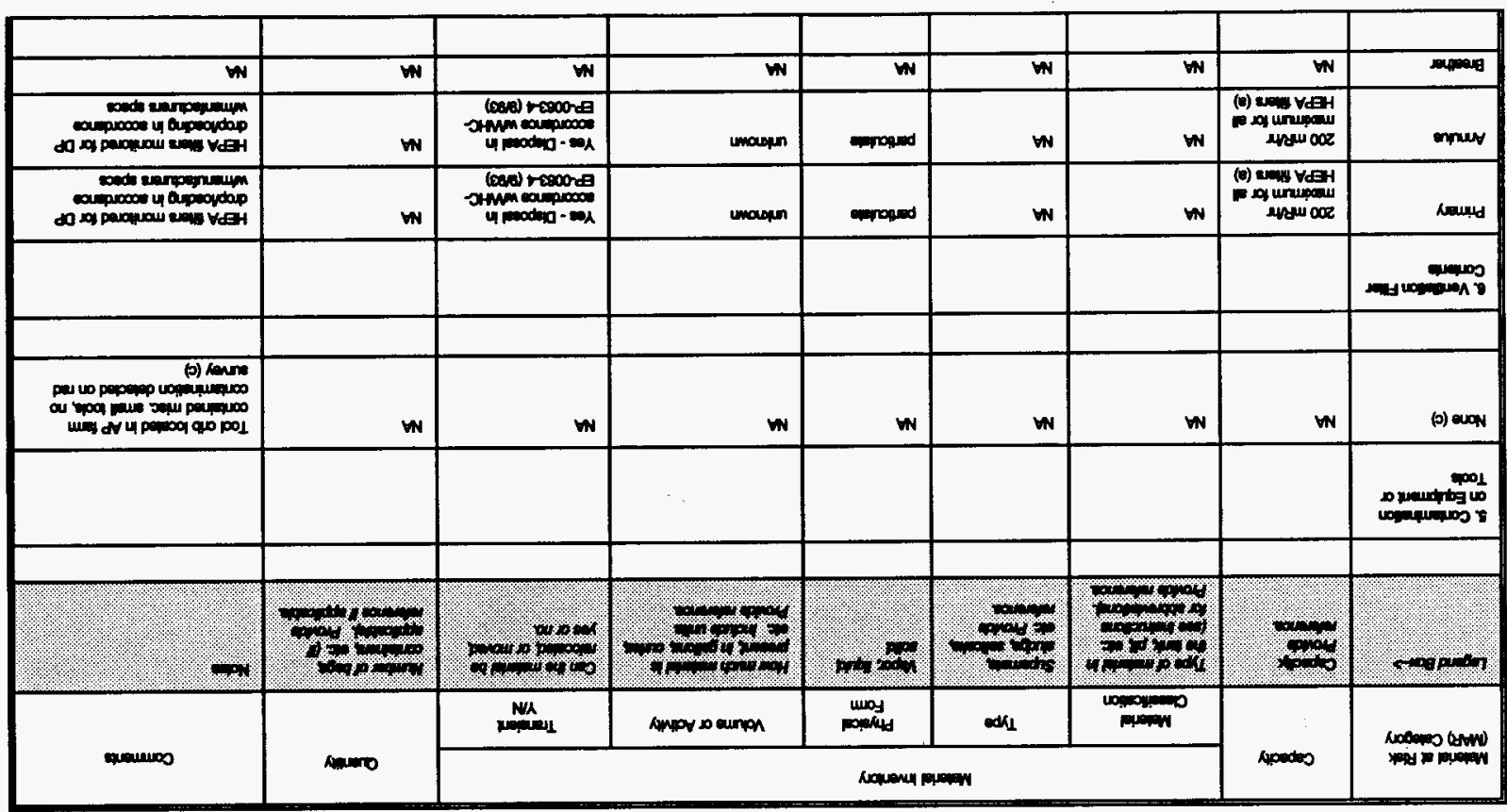

Noolvew stralm

I IIEVL 


\begin{tabular}{|c|c|c|c|c|c|c|c|c|}
\hline 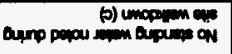 & $\mathbf{w}$ & $\mathbf{w}$ & $w$ & proty & w & ת & $\mathbf{w}$ & dune werowes \\
\hline & & & & & & & & : \\
\hline 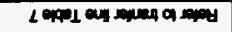 & $\bar{w}$ & $w$ & $w$ & $w$ & $w$ & $w$ & W & $\mathbf{W}$ \\
\hline & & & & & & & & 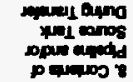 \\
\hline W & $\mathbf{W}$ & $\mathbf{w}$ & (p) mpopos & ponto & $\mathbf{w}$ & 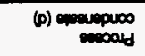 & W & doo moss \\
\hline $\mathbf{w}$ & $\mathbf{W}$ & $\mathbf{w}$ & (p) monsm & porby & w & (p) & W & 20109000000 \\
\hline & & & & & & & & 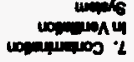 \\
\hline 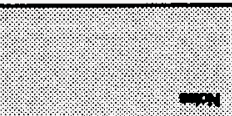 & 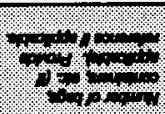 & (4) & $\int_{1}$ & $1+4 \%$ & 10 & 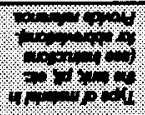 & 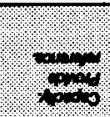 & 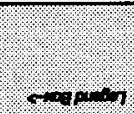 \\
\hline \multirow{2}{*}{ Amanios } & \multirow{2}{*}{ ONד } & mas & Ampor do cuning & $\operatorname{mog}_{1004}$ & $\operatorname{cod} K_{1}$ & Ding & \multirow{2}{*}{ Apoutes } & \multirow{2}{*}{ 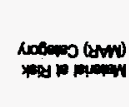 } \\
\hline & & \multicolumn{5}{|c|}{ 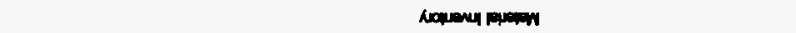 } & & \\
\hline
\end{tabular}




\begin{tabular}{|c|c|c|c|c|c|c|c|c|}
\hline \multirow{2}{*}{$\begin{array}{l}\text { Maluriat at Riok } \\
\text { (MAR) Cotopony }\end{array}$} & \multirow{2}{*}{ Cenpecily } & \multicolumn{5}{|c|}{ Mnteriel invertery } & \multirow{2}{*}{ avority } & \multirow{2}{*}{ Camments } \\
\hline & & Cimbificition & Type & Form & Wolume of Acivily & Tranient & & \\
\hline 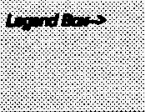 & $\operatorname{lom}_{1+\infty}$ & $\frac{1+1 \%}{1+1 \%}$ & कath & 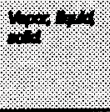 & 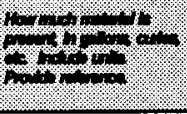 & 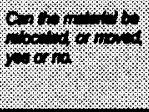 & $\frac{1}{1 \%}$ & \%, \\
\hline \multicolumn{9}{|l|}{ 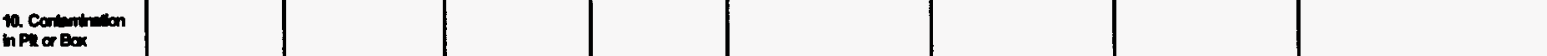 } \\
\hline Proceses pits & MA & recictud & M & recides & uninown & no & MA & No tending weiter nolad (e) \\
\hline 241 AP Vatio pit & $-21.5 \mathrm{Kgal}$ (d) & recidtal & M & reaches & unknown & no & $\begin{array}{l}1 \text { contrat whe pit o } \\
\text { AP }\end{array}$ & $M$ \\
\hline $\begin{array}{l}\text { Central Pump Pit } \\
(101-106)\end{array}$ & $-5.4 \mathrm{Kgal}(0)$ & pocidum & $\mathrm{M}$ & resides & unloom & no & opta lotel (1 pit por & $m$ \\
\hline \multicolumn{9}{|l|}{ 11. ENA 313} \\
\hline 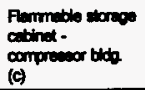 & $M$ & M & M & Haide & Untrown & 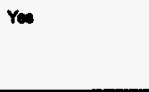 & $m$ & 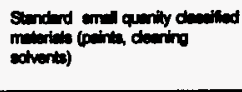 \\
\hline 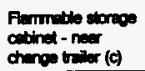 & MA & MA & $M$ & Lquith & Untrown & You & $M$ & 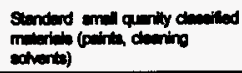 \\
\hline 12 Otw & & & & & & & & \\
\hline
\end{tabular}

NA = Not applicable or not required 


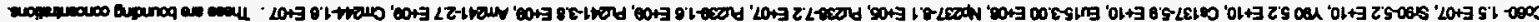

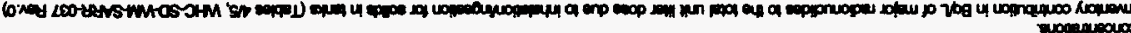

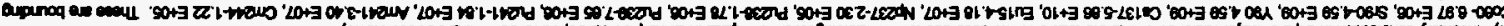

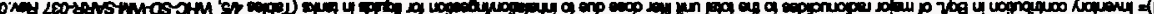

\begin{tabular}{|c|c|c|c|c|c|c|c|c|}
\hline 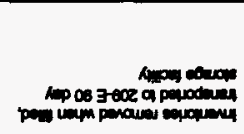 & 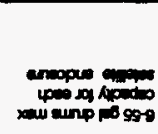 & $\omega$ & 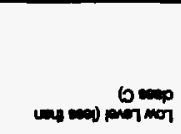 & Pys & 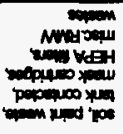 & pexis vesey & 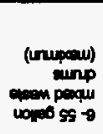 & mo vogernurooe \\
\hline 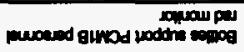 & $=9092$ & $\boldsymbol{\omega}$ & cmoq ad at ont & $\infty$ & $\mathbf{W}$ & $\begin{array}{r}\text { uoby } \% 06 \\
\text { ampeur } \% 01\end{array}$ & $\sin 2$ & 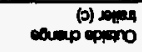 \\
\hline 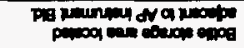 & $\operatorname{lom} 6$ & $\omega$ & $\operatorname{com}$ and as opt & $\infty$ & $\mathbf{w}$ & 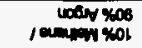 & $\operatorname{compa~th}$ & \\
\hline 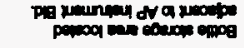 & $\operatorname{mog}$ & மג & empa ad ar oot & $\infty$ & $w$ & XN 태 / \% & sempa th & \\
\hline 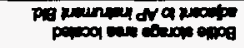 & $\operatorname{cop} 1$ & •人 & 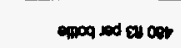 & $\infty$ & $\mathbf{W}$ & useaplyt & $\operatorname{com} m 0 \mathrm{a}$ & \\
\hline 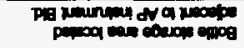 & $\cos x$ & $\boldsymbol{\omega}$ & ompa ad as cost & $=0$ & $w$ & netagry & $\begin{array}{r}\text { (xaul) } \\
\operatorname{spmoq} 8 z\end{array}$ & 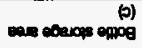 \\
\hline 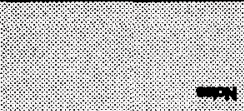 & 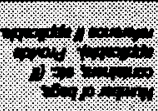 & 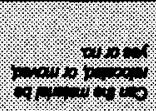 & 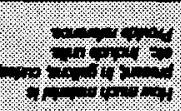 & 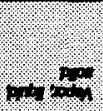 & $\operatorname{lom}_{10}$ & 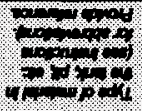 & $x \rightarrow$ & 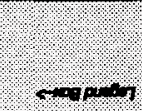 \\
\hline \multirow{2}{*}{ prounos } & \multirow{2}{*}{ A } & $\operatorname{NaA}_{1}$ & Amprofountor & mog & $e_{1}$ & mogromingon & \multirow{2}{*}{ Apodtos } & \multirow{2}{*}{ 1 } \\
\hline & & \multicolumn{5}{|c|}{ 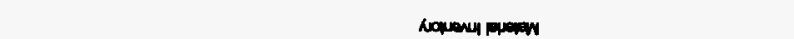 } & & \\
\hline
\end{tabular}

D. Aoy

100-3IH.WM as-5HM

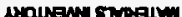

I ITIN 


$$
\begin{aligned}
& 1 \\
& 1 \\
& 111
\end{aligned}
$$


TAELE 2

\begin{tabular}{|c|c|c|c|c|c|c|c|c|c|c|c|c|c|c|c|}
\hline Facility & Type & Conceine & \begin{tabular}{|c|} 
B \\
Exploina \\
Pyrophoric
\end{tabular} & $\begin{array}{l}\text { C- } \\
\text { Nucloury } \\
\text { Citicatity }\end{array}$ & Fanmedo & $\begin{array}{c}\text { E- } \\
\text { Thermed } \\
\text { Puovision }\end{array}$ & $\begin{array}{l}\text { F- } \\
\text { Chem. } \\
\text { Potm }\end{array}$ & $\begin{array}{c}\text { G } \\
\text { Friction }\end{array}$ & Eedticul & 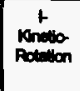 & Snito & $\underset{\substack{\text { Cranky, } \\
\text { Hoight }}}{K}$ & $\begin{array}{l}\text { L- } \\
\text { Preacers } \\
\text { Votume }\end{array}$ & 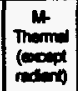 & Comments \\
\hline \multicolumn{2}{|c|}{ 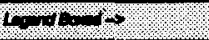 } & \multicolumn{13}{|c|}{ 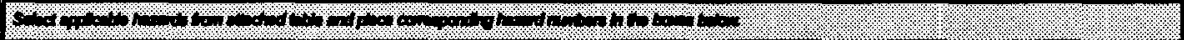 } & $\%$ \\
\hline \multicolumn{2}{|c|}{ NP TAKKFARM } & $\mathrm{MA}$ & MA & $\mathrm{MA}$ & $\mathrm{M}$ & $\mathrm{MA}$ & $M$ & $5(G)$ & $8(6)$ & $2,3(6)$ & $M$ & $11(C)$ & $M$ & MA & $M$ \\
\hline 241-AP-101 & CST & $2(a)$ & 6, 7, 8 (a) & $15(0)$ & $4,11,14(0)$ & $5^{\circ}(0)$ & MA(a) & $5(c)$ & 2, 14 (c) & 3 & $M(c)$ & $\operatorname{Ma}(c)$ & $M A(c)$ & $\mathrm{MA}(\mathrm{c})$ & Terk wasco (b) \\
\hline $241-19-102$ & DST & $2(a)$ & $6,7,8$ (a) & $15(0)$ & $4,11,14(a)$ & $5 *(a)$ & $M(a)$ & $5(\mathrm{G}$ & 9, 14 (c) & 3 & $M A(G)$ & $M(\theta)$ & $M(c)$ & MA(c) & Terk weme (b) \\
\hline $241-4-100$ & DST & $2(a)$ & $6,7,8$ (a) & $15(0)$ & $4,11,14(9)$ & $5^{\circ}$ (a) & MA(0) & $5(c)$ & 8,14 (c) & $3(4)$ & $m(c)$ & MA (c) & MA (c) & MA(c) & Therk weaca (b) \\
\hline 2A1-AP-104 & DST & $2(0)$ & $6,7,8(a)$ & $15(8)$ & $4,11,14(0)$ & $5(a)$ & $M A(0)$ & $5(C)$ & 0,14 (c) & $3(c)$ & MA (c) & $M A(G)$ & $M(c)$ & MA(c) & Tenk wase (b) \\
\hline 241-AP-106 & DST & 2 (a) & $6,7,8(a)$ & $15(0)$ & $4,11,14(a)$ & $\sigma^{*}(0)$ & $M(0)$ & $5(G)$ & 9. 14 (c) & $3(9)$ & MA(G) & $M(c)$ & $M(c)$ & $M(c)$ & Therk wemos (b) \\
\hline 241-AP-106 & DST & $2(a)$ & $6,7,8(a)$ & $15(0)$ & $4,11,14(a)$ & $s^{2}(a)$ & $M A(a)$ & $5(c)$ & $9,14(9)$ & $3(9)$ & $M(c)$ & $M(c)$ & $M(c)$ & MA(c) & Therk vante (b) \\
\hline $241-4 P-107$ & DST & $2(a)$ & $6,7,8(a)$ & $15(a)$ & $4,11,14(n)$ & $\sigma^{\circ}(a)$ & MA(a) & $5(C)$ & 9,14 (c) & $3($ c) & $M(C)$ & $M(C)$ & M (c) & NA(c) & Thrt wame (b) \\
\hline 241-AP-100 & DST & $2(a)$ & $6,7,6(a)$ & $15(\mathrm{~s})$ & $4,11,14(0)$ & $s(0)$ & NA (a) & $5(c)$ & 9,14 (c) & 3 (c) & $M$ (c) & MA (c) & $M(c)$ & M(c) & Terk weich (b) \\
\hline $241 A^{P}$ & Vitue pat & 2 (b) & MA (b) & 15 (b) & MA (b) & MA(b) & $M(b)$ & 5 (b) & 14 (b) & MA(b) & $M(b)$ & $M(b)$ & $M(b)$ & M(b) & $M$ \\
\hline $\cos A P-010$ & $\begin{array}{l}\text { Coen al } \\
\text { box }\end{array}$ & $M$ & $M$ & MA & $\mathrm{MA}$ & NA & MA & MA & $M$ & MA & $M$ & $m$ & M & MA & $\begin{array}{l}\text { Nocosin in AP Fam } \\
\text { (b) }\end{array}$ \\
\hline COBAP-OTE & Cloen out & MA & MA & MA & $M$ & $\mathrm{MA}$ & MA & $\mathrm{M}$ & $m$ & $M$ & $M$ & $\mathrm{~m}$ & MA & $\mathrm{MA}$ & $\mathrm{N}_{\text {(b) }} \operatorname{coses}$ in AP Fam \\
\hline COBAP-OTF & Coen ad & $M$ & MA & HA & M & M & MA & MA & $M$ & MA & $M$ & M & $M$ & $M$ & $\begin{array}{l}\text { No } \operatorname{coses} \text { h AP Fam } \\
\text { (b) }\end{array}$ \\
\hline $241-A$ & Fuch pit & $M(B)$ & NA(b) & MA (b) & NA (b) & $M A(b)$ & MA (b) & NA(b) & 14 (b) & MA (b) & $M A(O)$ & $M A(b)$ & $M(0)$ & MA (b) & leak demation in wump \\
\hline 2A1-AP-2T3 & Sorvice Bldp. & NA(c) & $M(c)$ & MA (c) & MA (c) & $\mathrm{MA}$ (c) & MA (C) & MA(C) & 11,14 (c) & Ma (c) & M (c) & $M$ (c) & MA(c) & 3 (c) & $\begin{array}{l}\text { Space hender in sentose } \\
\text { Eld. }\end{array}$ \\
\hline $241-49-020$ & $\begin{array}{l}\text { Feed purp } \\
\text { pit }\end{array}$ & 2 (b) & $M A(b)$ & 15 (b) & MA (0) & $M(b)$ & MA (b) & $5(c)$ & $9,14(c)$ & $3(c)$ & $M(c)$ & M (c) & $M(c)$ & $M(c)$ & M \\
\hline
\end{tabular}

NA $=$ Not applicable or not required

$5 / 31 / 96$ 


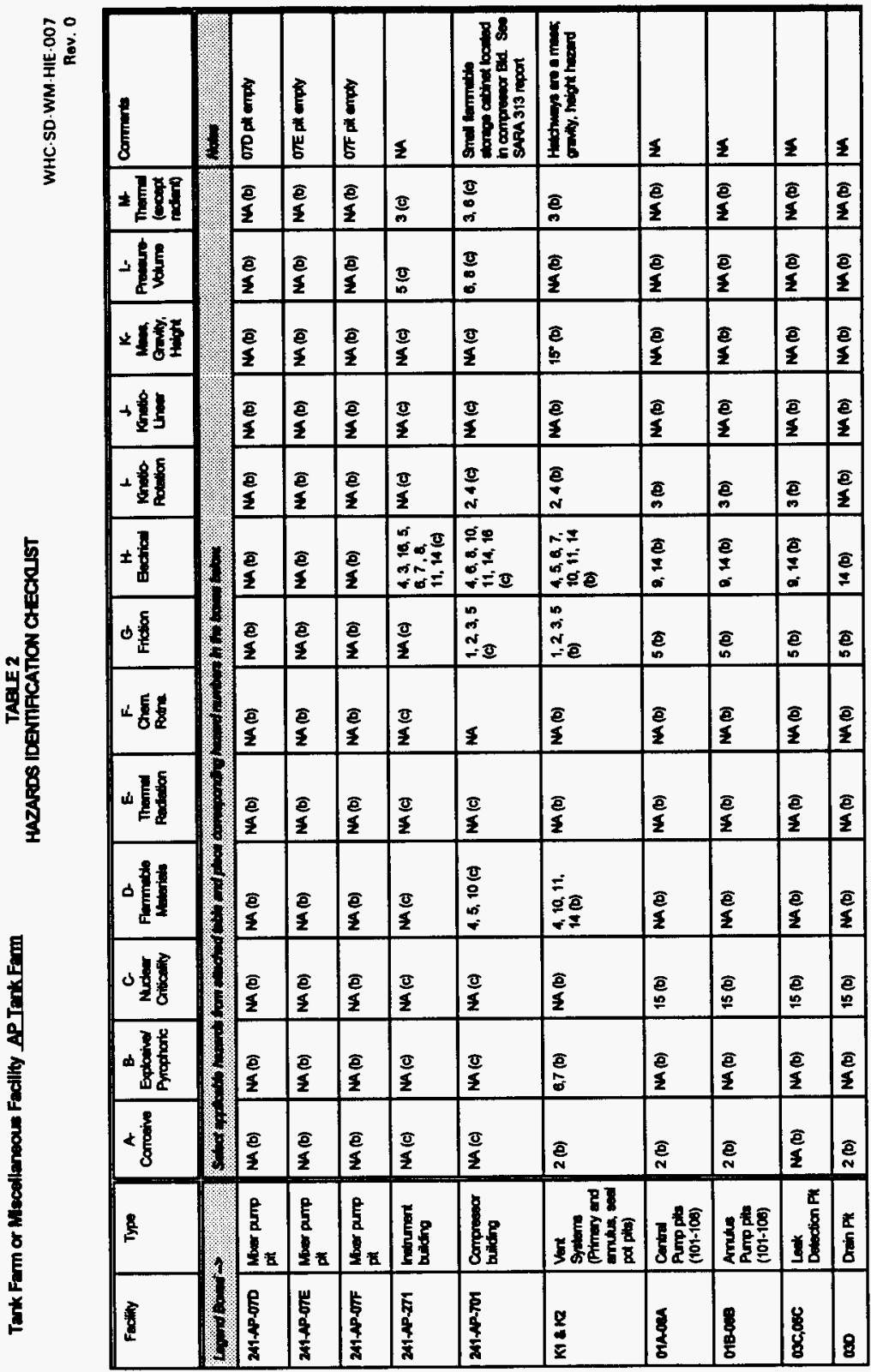

$\frac{R}{d}$ 


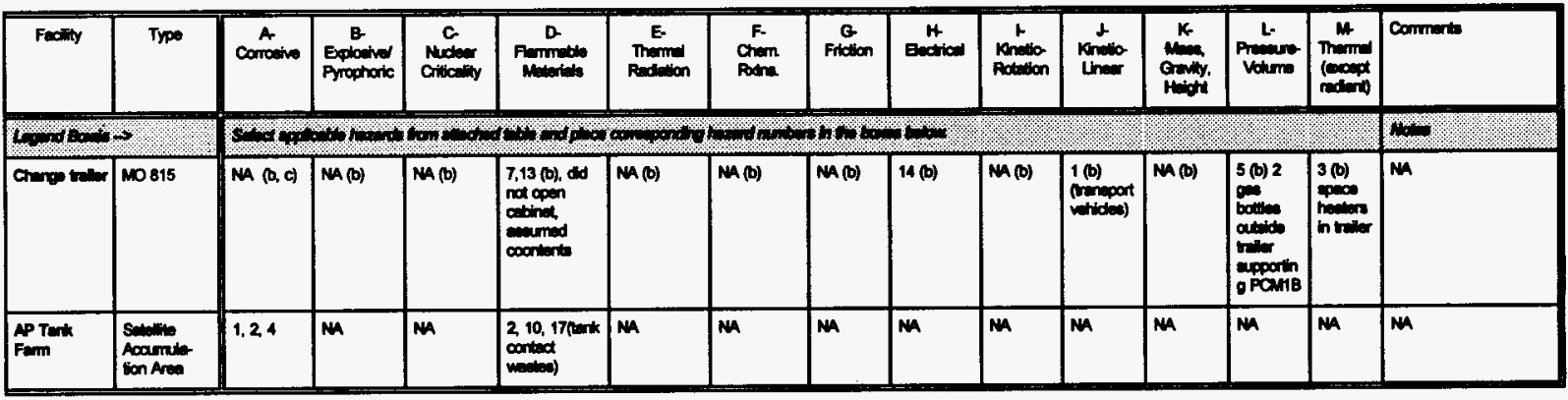

(a) $=080-T-15 \%-00007$

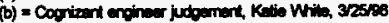

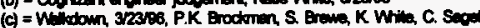

- All fran reforence b 
Table 3

Un'que Phencmena Chodkd

\begin{tabular}{|c|c|c|c|c|c|c|c|c|c|c|c|c|c|}
\hline \multirow{2}{*}{ Facilly } & \multirow[t]{2}{*}{ Type } & \multirow{2}{*}{$\begin{array}{c}\text { Flammable } \\
\text { Gess }\end{array}$} & \multirow[t]{2}{*}{ Fenrogyenide } & \multicolumn{3}{|c|}{ Openice } & \multirow{2}{*}{ 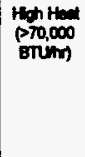 } & \multirow[t]{2}{*}{ Pyrephorics } & \multirow[t]{2}{*}{ Sphonim } & \multirow{2}{*}{ 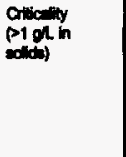 } & \multirow{2}{*}{ 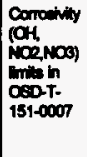 } & \multirow[t]{2}{*}{ Oher-1 } & \multirow[t]{2}{*}{ Comments } \\
\hline & & & & $\begin{array}{l}\text { Seperiblo } \\
\text { Phere }\end{array}$ & $\begin{array}{l}\text { roc in } \\
\text { liquile } \\
\text { p10 ol) }\end{array}$ & 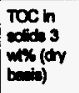 & & & & & & & \\
\hline \multicolumn{2}{|c|}{$6.1 \mathrm{H}=$} & $\frac{6,10}{6+1}$ & 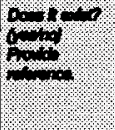 & $0^{6}$ & $\frac{0_{0}, 1}{6}$ & +1 & $\frac{1}{6}$ & $0 \%$ & $3 \%$ & $6 .+1 \%$ & \% & $\frac{1+1}{4}$ & $6 \%$ \\
\hline \multicolumn{2}{|c|}{ AP FARE } & & & & & & & & & & & & \\
\hline 241-NP-101 & DST & $\begin{array}{l}\text { N } \\
\text { Hodboon } \\
1903 \text { WHC. } \\
\text { SOWWER- } \\
\text { S28 Rev. } 1\end{array}$ & $\begin{array}{l}\text { N } \\
\text { Bormeim a } \\
\text { Simpeon te9t } \\
\text { WHCSDWh4 } \\
\text { ER-133 R. }\end{array}$ & $\begin{array}{l}\text { N } \\
\text { WHCSD } \\
\text { MuDP-161 } \\
\text { R1 }\end{array}$ & $\begin{array}{l}N \\
\text { Tum } \\
1900 \\
74430 \\
96-006\end{array}$ & Denot & $\begin{array}{l}\text { N } \\
\text { Tuler } \\
1900 \\
7440-80- \\
000\end{array}$ & 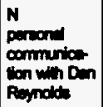 & 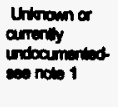 & 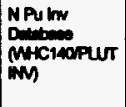 & $\begin{array}{l}\text { N } \\
\text { Trelor } \\
1006 \\
7400068 \\
008\end{array}$ & $M$ & 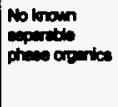 \\
\hline 241-AP-102 & DST & 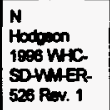 & 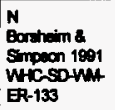 & $\begin{array}{l}\text { N } \\
\text { WHC-so } \\
\text { UMER-350 }\end{array}$ & 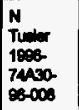 & Devenot & $\begin{array}{l}N \\
\text { Tunior } \\
1908 \\
7440068 \\
000\end{array}$ & 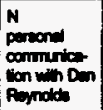 & 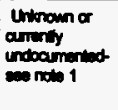 & 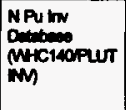 & $\begin{array}{l}N \\
\text { Tumer } \\
1006 \\
7440-88 \\
006\end{array}$ & NA & 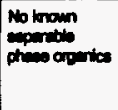 \\
\hline 241-AP-103 & DST & 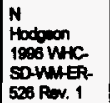 & $\begin{array}{l}\text { N } \\
\text { Bortheim a } \\
\text { Simpeon } 1991 \\
\text { WHCSD-WM } \\
\text { E-133 }\end{array}$ & $\begin{array}{l}\text { N } \\
\text { Whcso } \\
\text { Muter-4se }\end{array}$ & $\begin{array}{l}N \\
T \text { Tulas } \\
1980 \\
74930 \\
80-008\end{array}$ & Dedand & $\begin{array}{l}\text { N } \\
\text { Tumler } \\
1808 \\
74450.96 \\
000\end{array}$ & $\begin{array}{l}N \\
\text { poreorid } \\
\text { ocmminice } \\
\text { ton with Den } \\
\text { Repnolds }\end{array}$ & $\begin{array}{l}\text { Untroun or } \\
\text { ambanty } \\
\text { undoonmontat } \\
\text { see note } 1\end{array}$ & 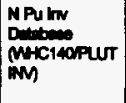 & $\begin{array}{l}\text { N } \\
\text { Tumer } \\
1006 \\
744008 \\
\text { cos }\end{array}$ & $\mathrm{NA}$ & $\begin{array}{l}\text { No known } \\
\text { phesto } \\
\text { orgerice }\end{array}$ \\
\hline
\end{tabular}


Table 3

Unique Phenomoma Checkst

\begin{tabular}{|c|c|c|c|c|c|c|c|c|c|c|c|c|c|}
\hline \multirow{2}{*}{ Facitity } & \multirow[t]{2}{*}{ Type } & \multirow{2}{*}{ Plominabe } & \multirow[t]{2}{*}{ Ferrogyenide } & \multicolumn{3}{|c|}{ Oronics } & \multirow{2}{*}{ 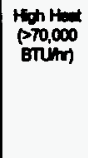 } & \multirow[t]{2}{*}{ Pyrapharica } & \multirow[t]{2}{*}{ Sphoring } & \multirow{2}{*}{ 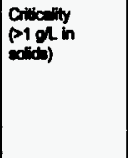 } & \multirow{2}{*}{ 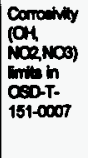 } & \multirow[t]{2}{*}{ Other-1 } & \multirow[t]{2}{*}{ Commerts } \\
\hline & & & & Sepentib & 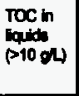 & $\begin{array}{l}\text { toc in } \\
\text { solls } 3 \\
\text { wo (dy } \\
\text { beis) }\end{array}$ & & & & & & & \\
\hline 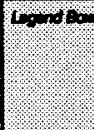 & & $\frac{6.14}{6 \%}=9$ & $\left.\frac{1}{1+9}\right)^{9}$ & 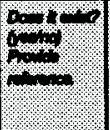 & $\frac{10,1}{6}$ & $\frac{1}{\infty}$ & 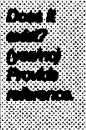 & ${ }^{1}$ & 10,14 & \% & 1. & $\frac{1}{6}$ & 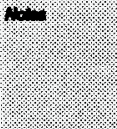 \\
\hline 241-4P-104 & DST & $\begin{array}{l}\text { N } \\
\text { Hodgan } \\
\text { Ises Witc- } \\
\text { SDWheR- } \\
528 \text { Rov. } 1\end{array}$ & $\begin{array}{l}\text { N } \\
\text { Boraheim \& } \\
\text { Simpeon } 1891 \\
\text { WHC-SDWN } \\
\text { ER-133 }\end{array}$ & $\begin{array}{l}\text { N } \\
\text { Siloy } 1906 \\
71720-95-000\end{array}$ & $\begin{array}{l}N \\
T \text { Tumes } \\
1996 \\
74400 \\
96-006\end{array}$ & D. & 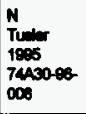 & 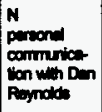 & 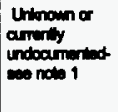 & $\begin{array}{l}\text { NPu hN } \\
\text { Dothem } \\
\text { MHC140HPUT } \\
\text { INM }\end{array}$ & $\begin{array}{l}\text { N } \\
\text { Tumer } \\
1908 \\
745068 \\
\text { ons }\end{array}$ & $M$ & 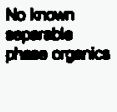 \\
\hline $241-A P-105$ & DST & 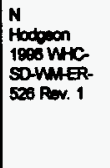 & $\begin{array}{l}\text { N } \\
\text { Borsheim \& } \\
\text { Simpeon } 1991 \\
\text { WHC-SOWh } \\
\text { ER-133 }\end{array}$ & $\begin{array}{l}N \\
\text { WHCSO } \\
\text { WHER } 390\end{array}$ & $\begin{array}{l}N \\
\text { Tumor } \\
1908 \\
74400 \\
86-006\end{array}$ & $\begin{array}{l}\text { Unlonoun } \\
\text { or } \\
\text { amirity } \\
\text { undoam } \\
\text { erted }\end{array}$ & $\begin{array}{l}N \\
\text { Tuler } \\
1908 \\
7440-86- \\
000\end{array}$ & $\begin{array}{l}\text { N } \\
\text { perandid } \\
\text { commitice } \\
\text { lion nith Den } \\
\text { Regnoth }\end{array}$ & 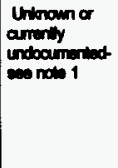 & 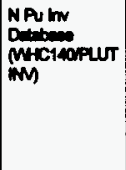 & 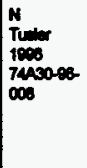 & 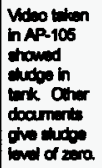 & $\begin{array}{l}\text { No known } \\
\text { pheme ordention }\end{array}$ \\
\hline 211AP-106 & CST & $\begin{array}{l}\text { N } \\
\text { Hodgon } \\
1000 \text { Wh } \\
\text { SDWwES } \\
520 \text { ReN. } 1\end{array}$ & $\begin{array}{l}\text { N } \\
\text { Bormaim a } \\
\text { Simpeon } 1991 \\
\text { WHA-SOWh } \\
\text { ER-133 }\end{array}$ & $\begin{array}{l}\text { N } \\
\text { WHCSO } \\
\text { WuOP-OTo } \\
\text { Ro }\end{array}$ & 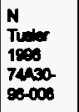 & Dan net & $\begin{array}{l}N \\
\text { Tumer } \\
1000 \\
740000- \\
000\end{array}$ & $\begin{array}{l}\text { N } \\
\text { persond } \\
\text { comminices. } \\
\text { bon with Dan } \\
\text { Romolde }\end{array}$ & 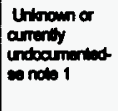 & 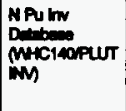 & $\begin{array}{l}N \\
\text { Tuenor } \\
\text { teos } \\
740000 \\
000\end{array}$ & $M$ & 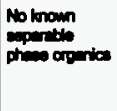 \\
\hline 2A1-AP-107 & DST & $\begin{array}{l}\text { N } \\
\text { Hodpoon } \\
1006 \text { WHC } \\
\text { SOWHER } \\
523 \text { Rov. } 1\end{array}$ & $\begin{array}{l}\text { N } \\
\text { Eontheim \& } \\
\text { Simpeon } 1991 \\
\text { WHC-SONW } \\
\text { ER-133 }\end{array}$ & $\begin{array}{l}\text { N } \\
\text { WHCSD } \\
\text { wuDP-0so } \\
\text { ROA }\end{array}$ & 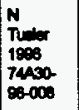 & Dita not & $\begin{array}{l}N \\
\text { Tumes } \\
1808 \\
74030-88 \\
000\end{array}$ & 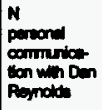 & 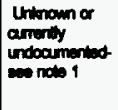 & 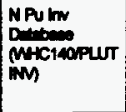 & 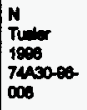 & $M$ & No known \\
\hline
\end{tabular}

NA = Not applicable or not required $5 / 31 / 56$ 


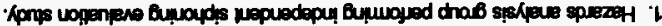

\begin{tabular}{|c|c|c|c|c|c|c|c|c|c|c|c|c|c|}
\hline 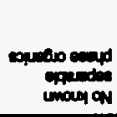 & $\mathbf{w}$ & 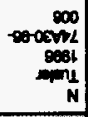 & 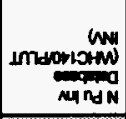 & 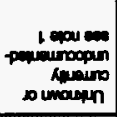 & 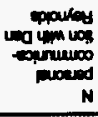 & $\begin{array}{r}\infty 00 \\
00072 \\
0081 \\
0911 \\
N\end{array}$ & and & 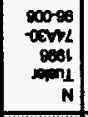 & $\begin{array}{r}0 y \\
\text { ostadomy } \\
\text { os }\end{array}$ & 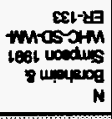 & 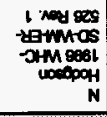 & 150 & col-drtik \\
\hline$\%$ & 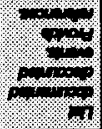 & 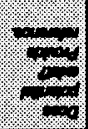 & 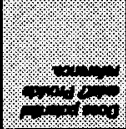 & ${ }^{2}$ & $\frac{1.96}{100}$ & .4 & . & . & 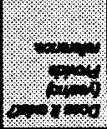 & (1) & 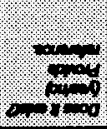 & \\
\hline & & $\begin{array}{r}2000-195 \\
-1-0950 \\
4\end{array}$ & & & & & 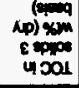 & 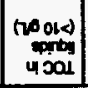 & anderdes & & & \multirow[b]{2}{*}{ II } & \multirow{2}{*}{ sod } \\
\hline סמש & | & (1) & & apoups & oproptoist & 14\% & \multicolumn{3}{|c|}{ Do } & andowas & & & \\
\hline
\end{tabular}

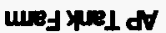

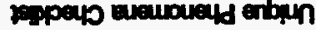
E eppl 


\begin{tabular}{|c|c|c|c|c|c|}
\hline$w$ & (b) 8 & (1) $n$ & (c) $\max$ & $w$ & 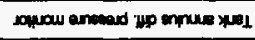 \\
\hline 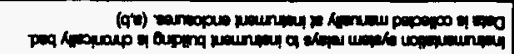 & pocturs & (A) & $(10 \operatorname{tat}) \cos$ & w & anseredual \\
\hline 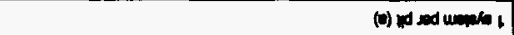 & ()) 8 & (a) $\max$ & $(4-5) \infty$ & $w$ & coppenep yog w dind \\
\hline (o) yaf ed unates & (0) 0 & $(\theta)=x$ & $(t)=x$ & $w$ & DNow \\
\hline 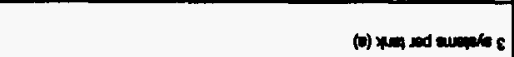 & (ค) 8 & $\theta=0$ & $(\theta \nabla)=0$ & $\mathbf{w}$ & 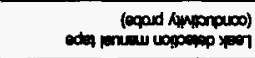 \\
\hline 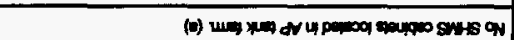 & $w$ & $w$ & $\mathbf{w}$ & $w$ & SWHS / Auroman wo \\
\hline 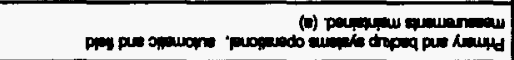 & (0) 8 & (o) $m$ & (0) $\operatorname{mox}$ & $w$ & 1 \\
\hline 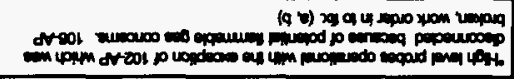 & (ค) 8 & $\operatorname{mou} 000(0) \cos$ & (0) $\operatorname{mox}$ & w & apod preq 40H \\
\hline$m$ & $w$ & $w$ & (q) $\infty$ & $w$ & 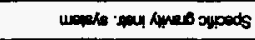 \\
\hline & & & & & 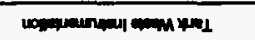 \\
\hline & & & & sol than & 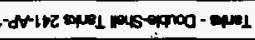 \\
\hline 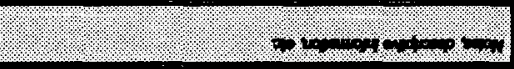 & 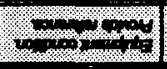 & 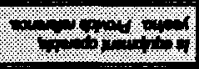 & 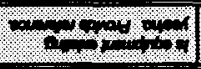 & 1,109 & $p=1$ \\
\hline numbos & compios raweritiog | & o & recodpanpos & 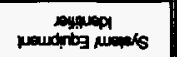 & wopers \\
\hline
\end{tabular}

$0.10 \mathrm{H}$

LOO-JIH-WM-OS-5HM

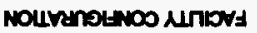

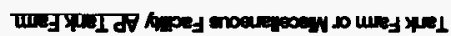


$96 / 1 \varepsilon s$

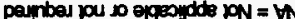

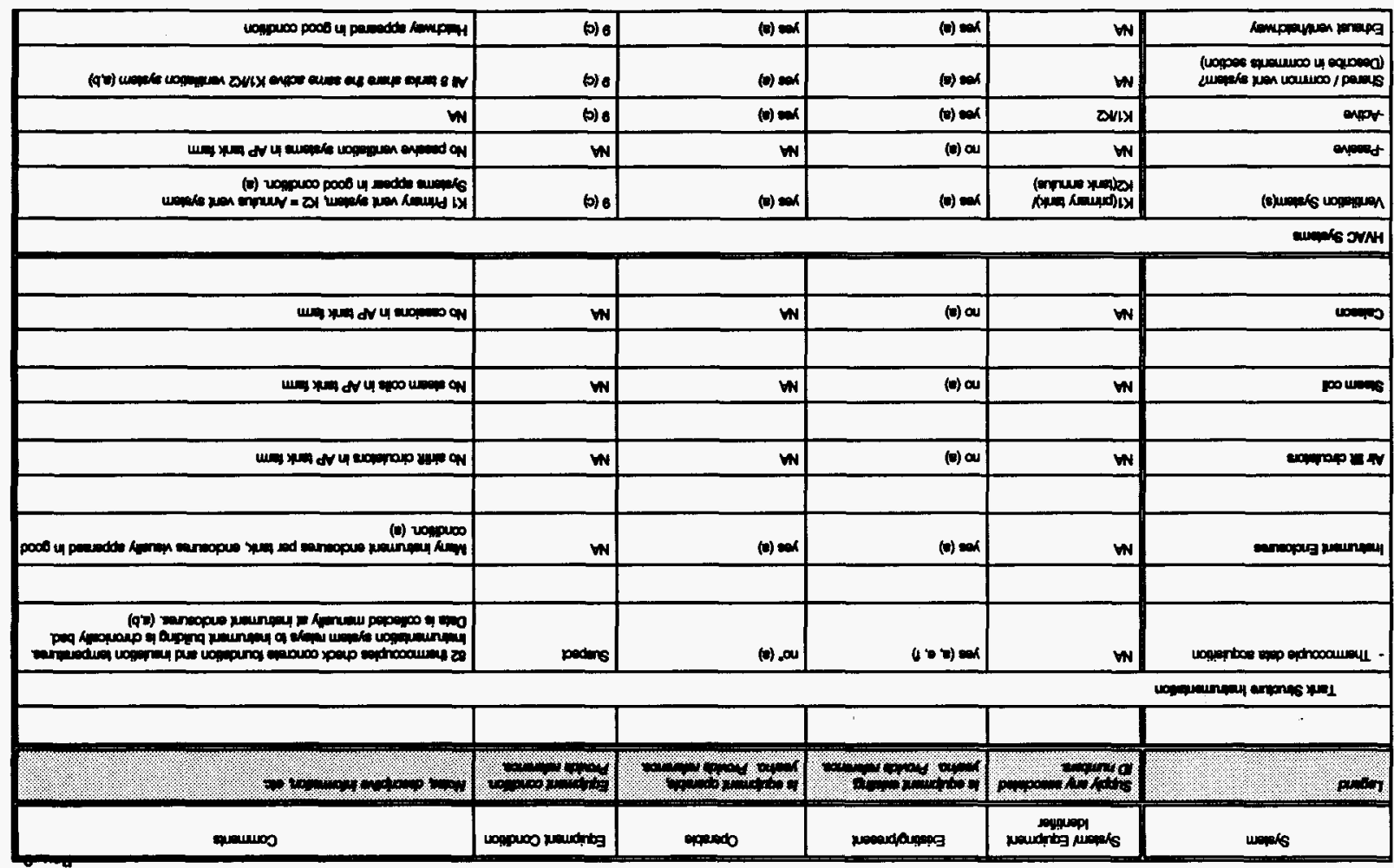

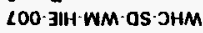

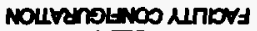

t ITav1 


\begin{tabular}{|c|c|c|c|c|c|}
\hline $\mathbf{w}$ & (b) 8 & (a) $=x$ & (B) $\cos$ & $1-t>1 \times 3)$ & newap pur \\
\hline$w$ & ()) 8 & (o) $\operatorname{sen}$ & (由) $\cos$ & $1-1 x=00$ & uopepp you durs \\
\hline w & $\mathbf{w}$ & (a) $=\infty$ & (b) $\operatorname{mox}$ & I-ly -d & dund dins \\
\hline$w$ & $\mathbf{w}$ & $w$ & (c) $\infty$ & 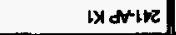 & 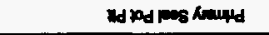 \\
\hline$w$ & ()) 0 & (1) $=\mathbf{m a}$ & (P) $\operatorname{eac}$ & mat ox & anomad epsos mpury \\
\hline$m$ & (6) & (t) 201 & (0) $\cos$ & anaw ix & 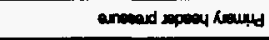 \\
\hline 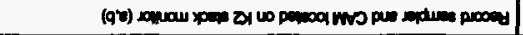 & (p) 8 & (a) $=0$ & (b) $\infty$ & man का & conow wats \\
\hline 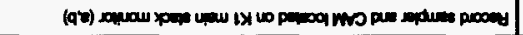 & (๑) 8 & (0) $\infty$ & (0) $\infty$ & 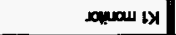 & 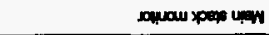 \\
\hline \multicolumn{6}{|r|}{ 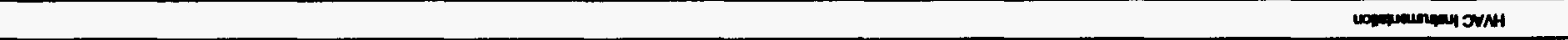 } \\
\hline 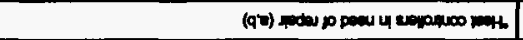 & podens & (0). $\operatorname{sen}$ & (s) $\max$ & क्ञpa เ्र & 4 \\
\hline 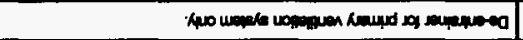 & (0) 6 & $(0)=1$ & (0) $\operatorname{eos}$ & Aq⿴囗十 เ्र & soupenuog \\
\hline$w$ & (p) 6 & $(0)=x$ & (0) $m$ & क्र pae เ्र & sed \\
\hline 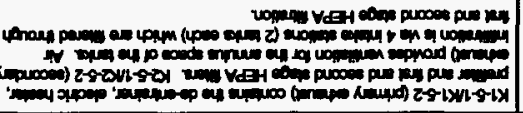 & (ค) 6 & (o) $\infty 1$ & (d) $\cos$ & Q WNLX & 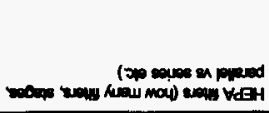 \\
\hline$w$ & (b) 6 & (q) $=0 \times$ & (b) $=x$ & $w$ & 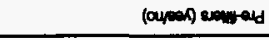 \\
\hline 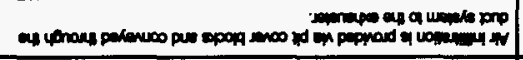 & $\mathbf{w}$ & $w$ & (0) ow & w & 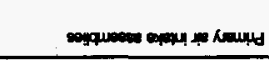 \\
\hline 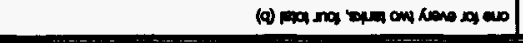 & (0) 8 & (v) $=\infty$ & (0) $\infty$ & $w$ & 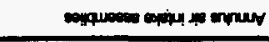 \\
\hline 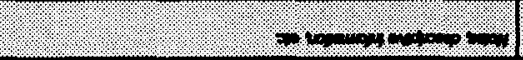 & $(2+\infty)$ & $4 \%$ & 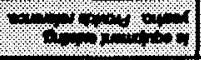 & $(1,0,19$ & 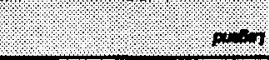 \\
\hline manus & 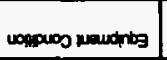 & rapodo & rooutoungepg & Mouditiog pinats & wowls \\
\hline
\end{tabular}




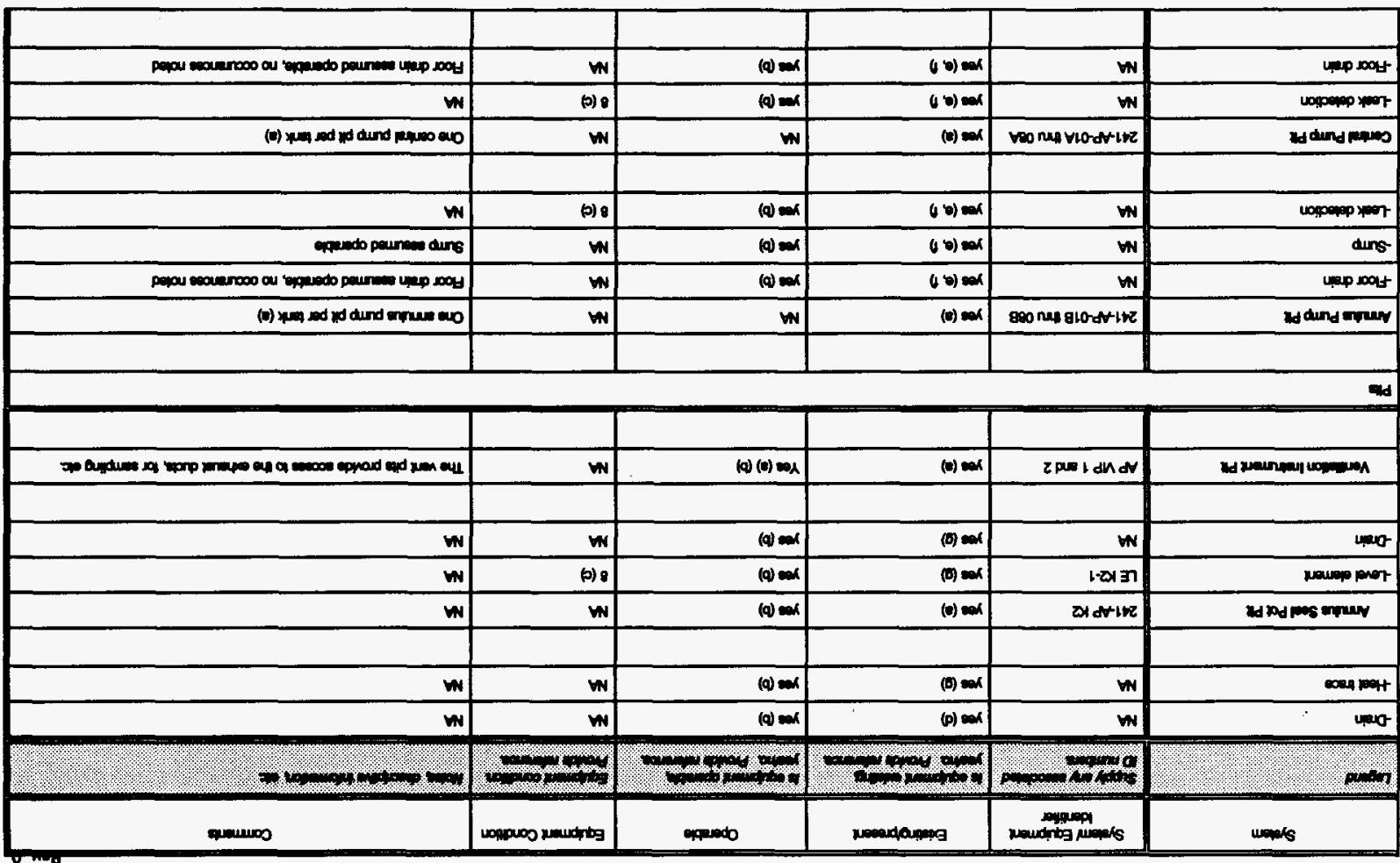




\begin{tabular}{|c|c|c|c|c|c|}
\hline $\mathbf{m}$ & $w$ & $w$ & (t) au & $w$ & Panpos \\
\hline w & $\mathbf{w}$ & w & (v) ou & w & $\sin x$ \\
\hline$w$ & $w$ & $(\phi)=0$ & 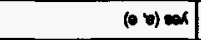 & w & $\operatorname{man}$ \\
\hline $\mathbf{w}$ & $\mathbf{w}$ & (q) $\cos$ & $(\theta \omega) \infty$ & w & ding \\
\hline 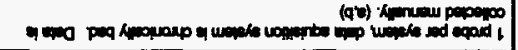 & podons & (b) & $(\theta) \cos$ & W & armendupl- \\
\hline $\mathbf{w}$ & (6) 8 & (b) $\max$ & (ov) $\cos$ & w & youpep par \\
\hline$w$ & (b) 8 & (o) $\cos$ & $(\theta+\theta) \cos$ & w & 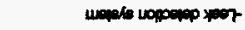 \\
\hline 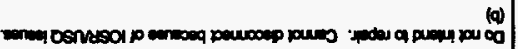 & 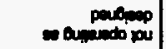 & (w) oul & $(0 v) \operatorname{cox}$ & $w$ & sonow pes anumer- \\
\hline 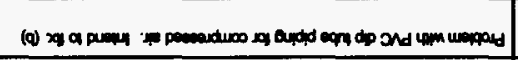 & 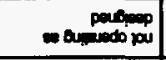 & (a) $\infty$ & $(0 \mathrm{n}) \cos$ & w & 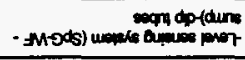 \\
\hline 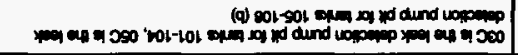 & $w$ & $w$ & (a) $\cos$ & 2000 & W dind lospapep yor \\
\hline $\mathbf{w}$ & $w$ & w & a) & w & am \\
\hline w & $w$ & $\mathbf{w}$ & a) & $w$ & Id explus \\
\hline 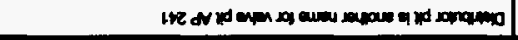 & $w$ & $w$ & (1) a) & $w$ & 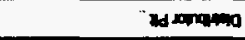 \\
\hline 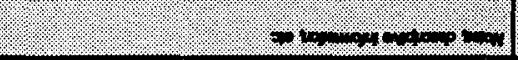 & $1 \%$ & 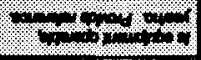 & 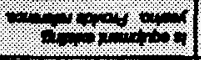 & r. & 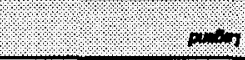 \\
\hline Manos & unpuos waucting & 9790000 & maxpanexg & 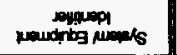 & wowers \\
\hline
\end{tabular}




\begin{tabular}{|c|c|c|c|c|c|}
\hline 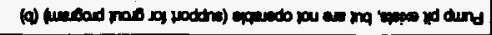 & $\mathbf{w}$ & (ब) \% & (0) $\max$ & Covodvitz & udund perey \\
\hline$w$ & (0) & (q) $\max$ & $\omega \infty \mathrm{N}$ & $\mathbf{w}$ & 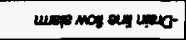 \\
\hline$w$ & (0) 8 & (Q) $\max$ & 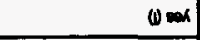 & $\mathbf{w}$ & mans anm \\
\hline w & $\mathbf{w}$ & $(q)=0$ & (D) $\max$ & olmocons & mass \\
\hline $\mathbf{w}$ & w & (a) $\min$ & $(a) \cos$ & otceoses & mis- \\
\hline $\mathbf{w}$ & $\mathbf{w}$ & $(q)=1$ & (b) $\operatorname{ex}$ & $\mathbf{w}$ & teat \\
\hline $\mathbf{w}$ & w & $\mathbf{m}$ & (e) ou & w & 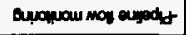 \\
\hline 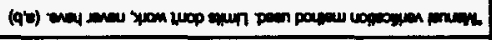 & & (1) sow & (0) $\operatorname{mos}$ & $w$ & 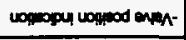 \\
\hline $\mathbf{w}$ & (b) 8 & (c) $\max$ & (D) $\max$ & $\mathbf{w}$ & uxpemp xpor \\
\hline $\mathbf{w}$ & w & $\mathbf{w}$ & (0) $\max$ & Alik & adern \\
\hline$w$ & (ค) 8 & $(q) \max$ & (d) $\infty$ & $w$ & compenp xor \\
\hline$w$ & (6) 8 & (a) $\operatorname{mox}$ & (d) $\max$ & $\mathbf{w}$ & na- \\
\hline 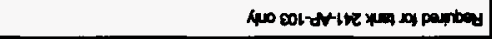 & $\mathbf{w}$ & w & (1) $\operatorname{mos}$ & 000 & Fil una \\
\hline$w$ & $w$ & $\mathbf{w}$ & (t) $\infty$ & w & yderd mines \\
\hline $\mathbf{w}$ & $\mathbf{w}$ & $\mathbf{w}$ & (v) ow & $w$ & Find anderse \\
\hline 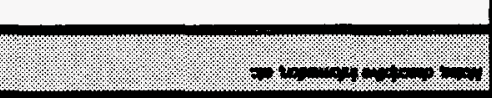 & 1.2\% & \% & 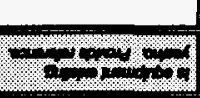 & (1.8. & 8 \\
\hline 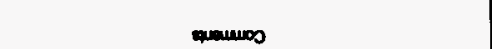 & 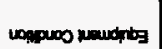 & 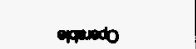 & rosadanges & 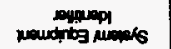 & manels \\
\hline
\end{tabular}


96IES partbes pu so apgoydide ow $=\forall N$

\begin{tabular}{|c|c|c|c|c|c|}
\hline upden (ransh) \&o \& & $\mathbf{w}$ & (Q) $\operatorname{\omega ac}$ & $(10) \mathrm{man}$ & $\mathbf{W}$ & tar \\
\hline $\mathbf{w}$ & (b) 8 & (q) $\cos$ & $(10)=1$ & $\mathbf{W}$ & 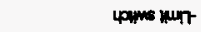 \\
\hline$w$ & ()) 8 & $(q-\cos )$ & (1) & $\mathbf{w}$ & Youne anmead int \\
\hline$m$ & (0) 8 & $(a-\operatorname{sen}$ & $(x)$ & $\mathbf{w}$ & Logpenep yor \\
\hline 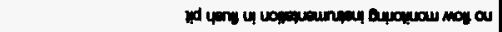 & $w$ & $\mathbf{w}$ & (b) $\infty$ & $\mathbf{w}$ & Aycquan mots \\
\hline $\mathbf{w}$ & $\mathbf{w}$ & (0) $\mathrm{eac}$ & (0) $=x$ & $d v i k$ & d t \\
\hline 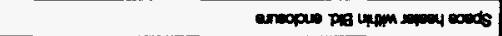 & $\mathbf{w}$ & (i) $\operatorname{sen}$ & $(x)=0$ & $\mathbf{W}$ & sensert \\
\hline $\mathbf{m}$ & $\mathbf{w}$ & (E) $\infty$ & $(n+D)=a$ & $\mathbf{w}$ & uant \\
\hline $\mathbf{w}$ & $w$ & (t) $\operatorname{ex}$ & $(1+0)=0$ & $\mathbf{W}$ & duns \\
\hline$m$ & (b) 9 & (t) $=\mathrm{ax}$ & $(100)=0$ & $\mathbf{W}$ & wertos ancout \\
\hline $\mathbf{W}$ & (6) 8 & (D) $\operatorname{man}$ & $(40 y) \operatorname{cox}$ & $\mathbf{W}$ & navep moldurs \\
\hline$w$ & (b) 8 & (b) $\max$ & $(4) \mathrm{b}$ & $\mathbf{W}$ & unpopip yor \\
\hline 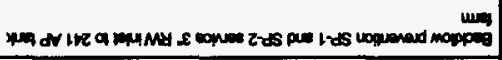 & $w$ & (t) $\cos$ & $(40)+x$ & $\mathbf{W}$ & moned moppes \\
\hline $\mathbf{w}$ & (5) 9 & (0) $\cos$ & $(x+8) \mathrm{cos}$ & WN & monow preyt \\
\hline w & ()) 8 & (0) $\mathrm{man}$ & $(40)=0$ & w & Ayponsoun mot \\
\hline & & & & & 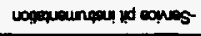 \\
\hline 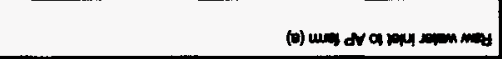 & (0) 8 & (a) $\operatorname{sen}$ & (b) $\operatorname{ean}$ & 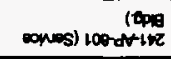 & ad connes \\
\hline 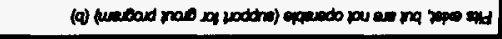 & $\mathbf{w}$ & (q) $q$ & (b) $\infty$ & \pm 203100 & id dind \\
\hline 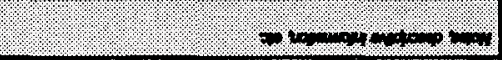 & \%, & \% & 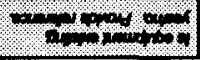 & 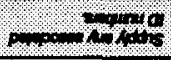 & $\log 1$ \\
\hline quinios & uopros neuctiog & eqpedo & pecadpungers & 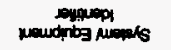 & unpers \\
\hline
\end{tabular}




\begin{tabular}{|c|c|c|c|c|c|}
\hline & & & & & 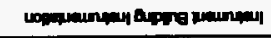 \\
\hline 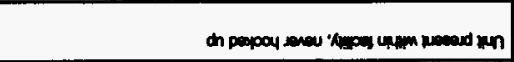 & $\mathbf{w}$ & (a t) a ou & (0) $\operatorname{sen}$ & w & 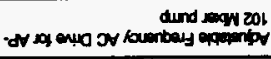 \\
\hline Apenq nopitad & $\mathbf{w}$ & $w$ & (t) $\operatorname{sen}$ & LLZATIR & apprig nounsain \\
\hline 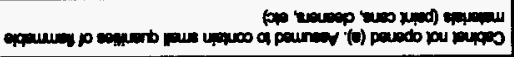 & $\mathbf{w}$ & $\mathbf{w}$ & (e) $\operatorname{man}$ & $\mathbf{w}$ & 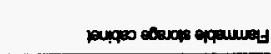 \\
\hline 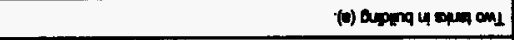 & w & (0) $\max$ & (a) $=0$ & w & spes appor \\
\hline \multirow[t]{2}{*}{ appompand } & w & $\mathbf{w}$ & (0) $\operatorname{sen}$ & boL-atike & avpere noesodicos \\
\hline & & & & & mpere \\
\hline 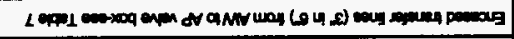 & $w$ & (Q) $\mathrm{max}$ & $\operatorname{ex}$ & (Mavodin) otivs & ant 1 \\
\hline 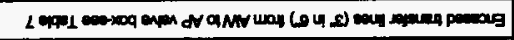 & $w$ & (q) $\operatorname{eac}$ & $\infty$ & 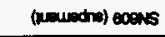 & ar \\
\hline 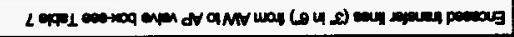 & $\mathbf{w}$ & (a) $\operatorname{cox}$ & $\operatorname{sex}$ & (kinp) olss & an wasell \\
\hline 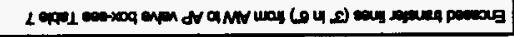 & $w$ & (q) $\operatorname{ear}$ & $\infty$ & (Ning) $\cos 75$ & an \\
\hline \multicolumn{6}{|r|}{ aptid degrall } \\
\hline $\mathbf{w}$ & $\mathbf{w}$ & $\mathbf{w}$ & (q) a & $\mathbf{W}$ & now unper \\
\hline 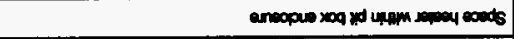 & $\mathbf{w}$ & $(9$ 4) & (⿻) & W & 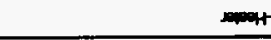 \\
\hline $\mathbf{w}$ & (6) 8 & $(q$ v) $m$ & (1) en & W & navp pret \\
\hline $\mathbf{W}$ & $\mathbf{w}$ & (a) $\operatorname{mos}$ & (1) $\operatorname{eac}$ & w & ang \\
\hline$w$ & $\mathbf{w}$ & (a) $\operatorname{man}$ & $0 \cos$ & m & duns \\
\hline 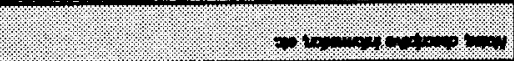 & 10 & 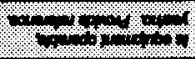 & $4+1 \%$ & 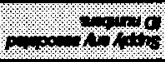 & 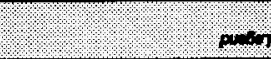 \\
\hline spallos & 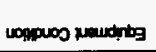 & axpedo & 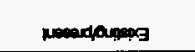 & 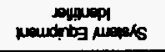 & movels \\
\hline
\end{tabular}




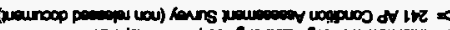

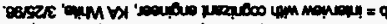

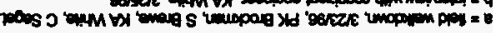

\begin{tabular}{|c|c|c|c|c|c|}
\hline 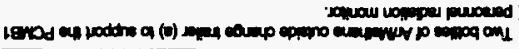 & $\mathbf{w}$ & (b) $=x$ & (b) $\operatorname{ex}$ & ste on & 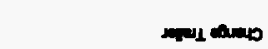 \\
\hline 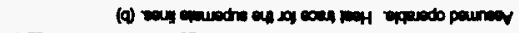 & $\mathbf{w}$ & (a) $\mathrm{max}$ & (b) $\cos$ & w & 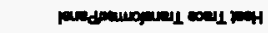 \\
\hline 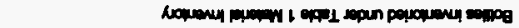 & $\mathbf{W}$ & w & (b) $\operatorname{ex}$ & $\mathbf{w}$ & 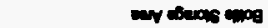 \\
\hline 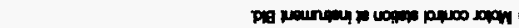 & (a) 8 & (c) $\mathrm{ex}$ & (c) $\mathrm{ex}$ & $A H E-20 N$ & $\log 15$ P \\
\hline 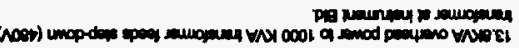 & (b) 0 & (C) $\sin$ & (b) $\cos$ & dRLE & wans maperg \\
\hline 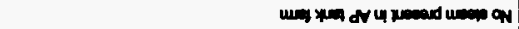 & $\mathbf{w}$ & $\mathbf{w}$ & $(q v)$ ou & $\mathbf{w}$ & chan uens \\
\hline 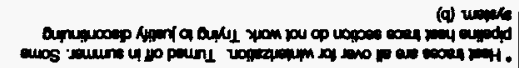 & $\mathbf{w}$ & (c) & (c) $\operatorname{sex}$ & $\mathbf{W}$ & 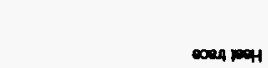 \\
\hline & & & & & 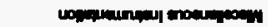 \\
\hline 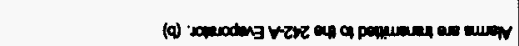 & $\mathbf{w}$ & (0) eas & $(t) \sec$ & $\mathbf{w}$ & 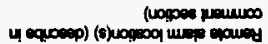 \\
\hline 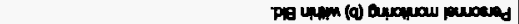 & $w$ & (0) $\mathrm{max}$ & (a) $=a x$ & $\mathbf{W}$ & $m$ \\
\hline 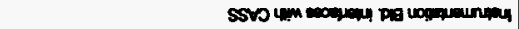 & (b) 8 & (a) $\cos$ & (q) $\operatorname{eac}$ & $\mathbf{W}$ & vopeunos SSP \\
\hline 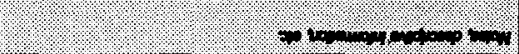 & 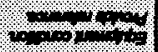 & 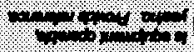 & $6 x_{2}+4$ & 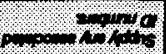 & 0,1 \\
\hline quavos & ucipuos Naxtrtog & epindo & pewadpapipg & poudditog popers & wemais \\
\hline
\end{tabular}

LOO-3IH-WM-OS-JHM

Nourenotros pnost

$\checkmark$ IIEN1 


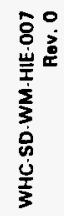

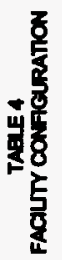

名

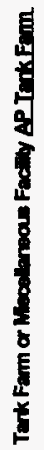

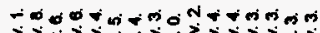

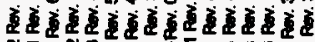

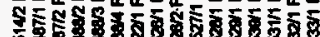




\begin{tabular}{|c|c|c|c|c|c|}
\hline $\mathbf{w}$ & w & $\mathbf{w}$ & $\mathbf{w}$ & A LKC ud arpen se enves & Pd \\
\hline (c) uonpusos poss & 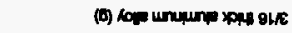 & (0) $:=0$ & $\mathbf{\alpha}$ & 2puldud & 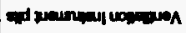 \\
\hline (t) ucapuos poses & 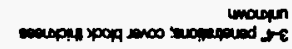 & (o) epopan mos & $\operatorname{sen}$ & $\pm N O=30-0 \angle 0-A+112$ & Pod dind man \\
\hline (c) Uonpuos poos & 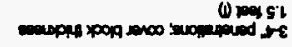 & (D) epong 100 & $\cos$ & $020-\forall 00-d N-I T Z$ & pd dund pery \\
\hline (b) inpos poos & (x) & (D) $=00 \times 19=0$ & $\cos$ & ardatiz & ad ad pene entiny \\
\hline (c) $100, p \times 00$ poses & 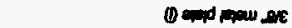 & (c) $200,7=0$ & $\operatorname{sen}$ & $b)=-1 b z$ & dod \\
\hline (1) U 100000000 & 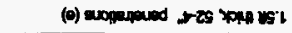 & (c) $\operatorname{spop}=100$ & $\infty$ & NAltz & ad expa \\
\hline (b) uonpos poss & 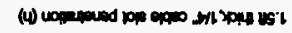 & 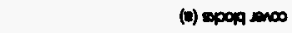 & $\operatorname{sex}$ & 020 & مصبا لمج \\
\hline or $4=0$ D & $\mathbf{w}$ & $\mathbf{w}$ & WN & $\mathbf{W}$ & ad dund nem ins \\
\hline ONu & W & $\mathbf{w}$ & $\mathbf{w}$ & $\mathbf{w}$ & ad dund apres \\
\hline 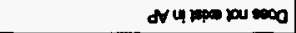 & $\mathbf{w}$ & w & W & $\mathbf{w}$ & d d \\
\hline (b) wompos poos & 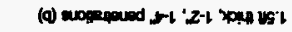 & (b) $\approx$ spop $=000$ & $\operatorname{sen}$ & 2901000 & d dund uopoup xim \\
\hline ov 4 ind pou & w & $\mathbf{w}$ & $w$ & $\mathbf{w}$ & adimy \\
\hline 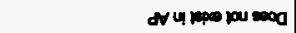 & $\mathbf{w}$ & $\mathbf{w}$ & w & W & H4 \\
\hline$\$ 4=000000$ & w & $\mathbf{w}$ & $\mathbf{w}$ & W & 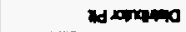 \\
\hline (c) 100 pios poos & 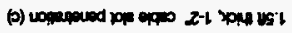 & 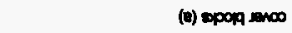 & $\operatorname{mox}$ & Vomatiodaltz & addund pepapes \\
\hline (b) 10000000000 & 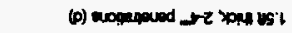 & 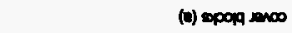 & $\operatorname{six}$ & 800 กया 8L0-d & addund many \\
\hline 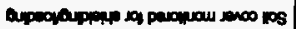 & 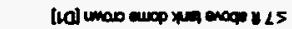 & enco punespos & $\operatorname{sen}$ & ool tenaus lol & 4 \\
\hline $4 \times$ & 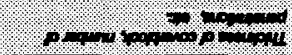 & 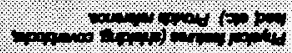 & 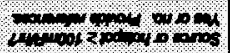 & 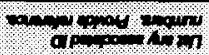 & $40,090,1$ \\
\hline mansos & 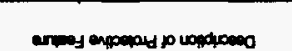 & anpey ansopald & 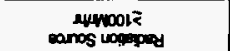 & of weipars & unats \\
\hline
\end{tabular}

0 AOY

200-3lH-wM-as כHM

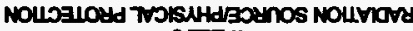

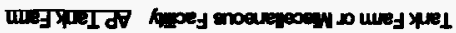


S6/LES

peupbea pu so opquydds pN $=\forall N$

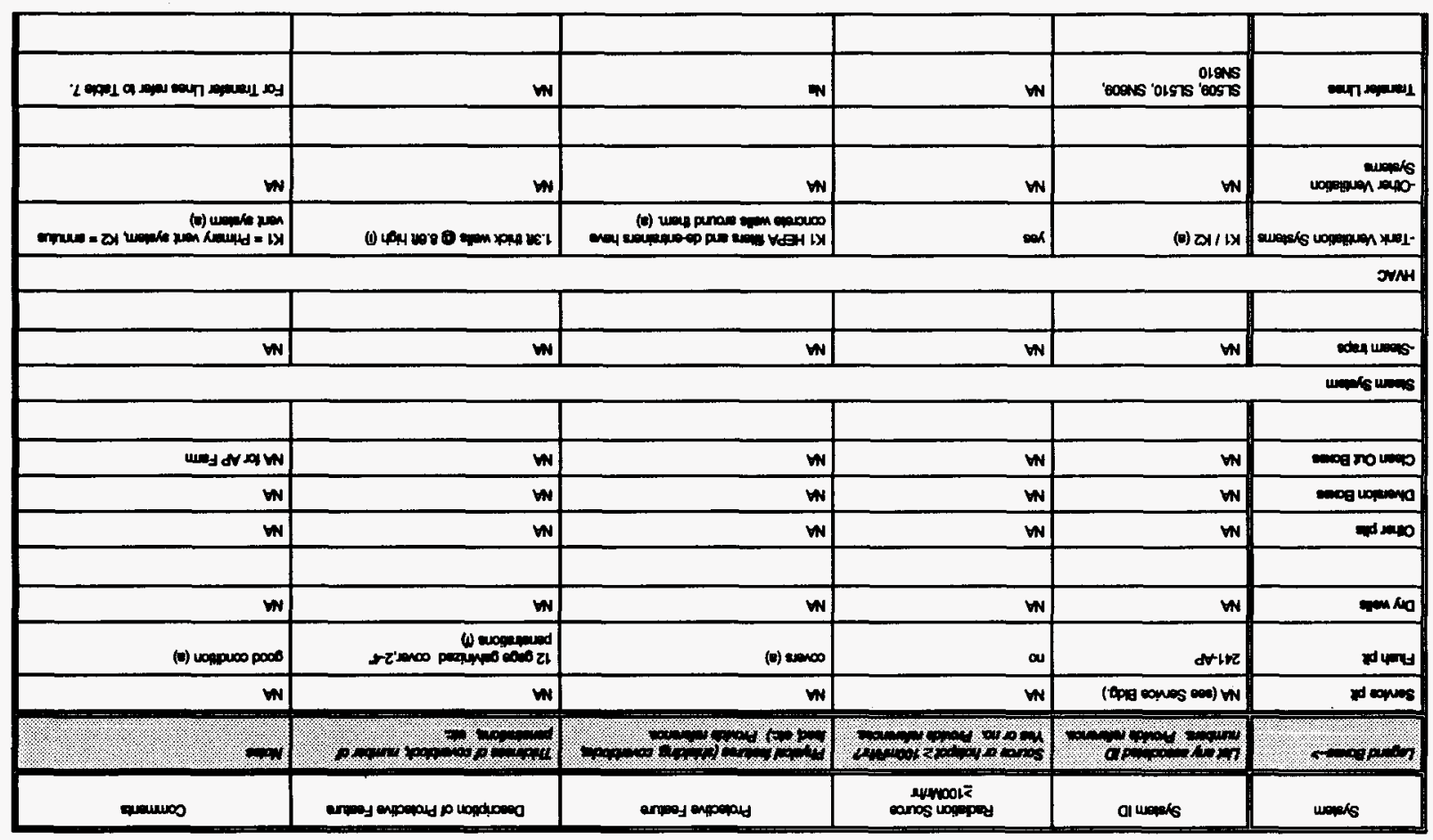

0 'ADY

100-ЭIH-WM-as-วHM
Nollagloud 7orshtedexanos nounary

S IISVI 
TAEE 5

\begin{tabular}{|c|c|c|c|c|c|}
\hline Syctem & Syatem ID & $\begin{array}{l}\text { Radition Sourco } \\
\geq 1000 \text { mh }\end{array}$ & Probctive Feature & Description of Provective Futur & Comments \\
\hline 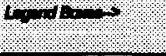 & $H_{12}+100$ & $\mathrm{H}_{0+1}+1$ & 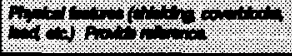 & 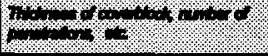 & $\%$ \\
\hline \multicolumn{6}{|l|}{ Alding } \\
\hline Chenge vivier & M & No & m & M & 1 cheres trisior \\
\hline $\begin{array}{l}\text { Senwime socamiation } \\
\text { area }\end{array}$ & M & $N o(\theta)$ & See cormment & M & 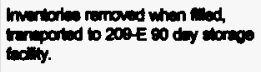 \\
\hline - Sonvios Buldiro & 241-AP-D01 (a) & no (a) & $M$ & M & 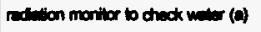 \\
\hline Compresor Buiding & 241AP-T01 (0) & no(a) & M & M & M \\
\hline Hrobument Bulding & $241-A P-271(0)$ & no (a) & MA & $M$ & CAM in buldng (o) \\
\hline
\end{tabular}

[01] CSOT-151-00007, Rov. H17

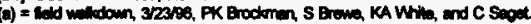

(b) = Drewing H2-6045

c)= Draing H-2-80446

Drong

(i) Drining 2 -

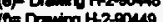

(1) Oranis He.

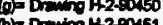

(i) $=$ Draning K $+2-00451$

(i) $=$ Draning $+2-9045$

O) Draning $+2-20035$

$(k)=$ Dreming H-2-6038

(1)= information from Ketio A whita, tune 1996. 
TABLE 6

Tank Farm or Miscellaneous Facility

AP

OSR/OSD SAFETY CONTROLS

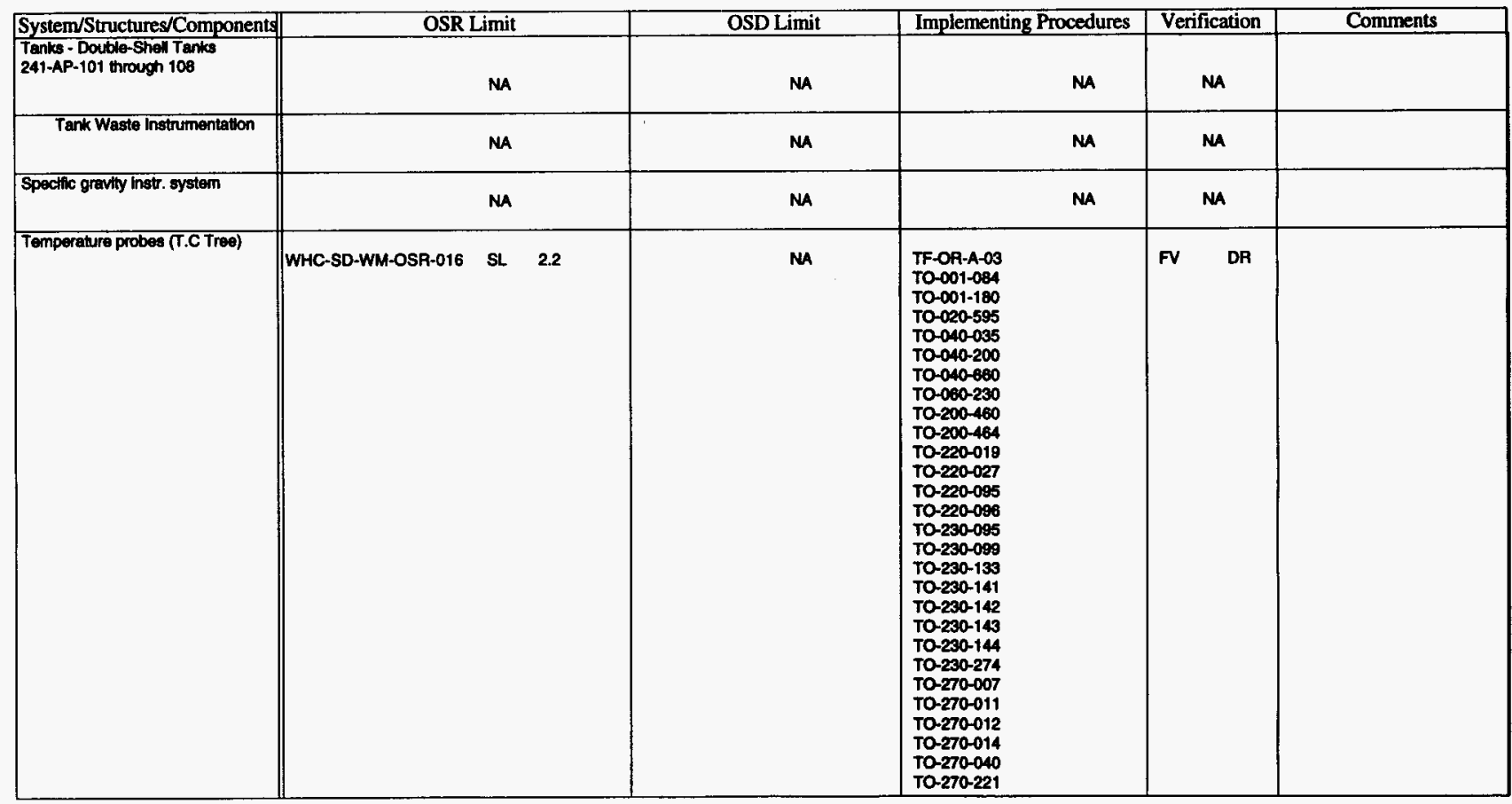

05/31/96

NA $=$ Not applicable or not required 
TABLE 6

Tank Farm or Miscellaneous Facility AP

OSR/OSD SAFETY CONTROLS

\begin{tabular}{|c|c|c|c|c|c|}
\hline System/Structures/Components & OSR Limit & OSD Limit & Implementing Procedures & Verification & Comments \\
\hline \multirow[t]{5}{*}{ 年 } & WHC-SD-WM-OSR-016 SL 2.2 & NA & $\begin{array}{l}\text { TO-270-235 } \\
\text { TO-270-251 } \\
\text { TO-270-252 } \\
\text { TO-270-266 } \\
\text { TO-270-824 } \\
\text { TO-400-200 } \\
\text { TO-430-480 } \\
\text { TO-430-485 } \\
\text { TO-440-002 }\end{array}$ & FV DA & \\
\hline & WHC-SD-WM-OSR-016 LCO 3.2 .1 & 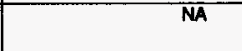 & NA & $F$ & $\begin{array}{l}\text { LCO-Not Implemented by } \\
\text { ECN }\end{array}$ \\
\hline & WHC-SD-WM-OSR-016 LCO $\quad 3.2 .2$ & NA & NA & NA & ECN 619396 \\
\hline & WHC-SD-WM-OSR-016 SR 3.2 .2 .1 & 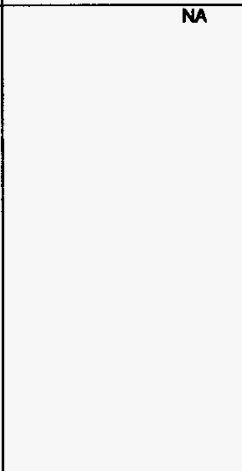 & $\begin{array}{l}\text { TF-OF-A-03 } \\
\text { TO-001-184 } \\
\text { TO-040-660 } \\
\text { TO-220-095 } \\
\text { TO-220-096 } \\
\text { TO-230-095 } \\
\text { TO-230-099 } \\
\text { TO-230-133 } \\
\text { TO-230-141 } \\
\text { TO-230-142 } \\
\text { TO-230-143 } \\
\text { TO-230-144 } \\
\text { TO-270-007 } \\
\text { TO-270-011 } \\
\text { TO-270-012 } \\
\text { TO-270-035 } \\
\text { TO-270-040 } \\
\text { TO-400-200 } \\
\text { TO-430-480 } \\
\text { TO-430-485 } \\
\text { TO-440-002 }\end{array}$ & FV DA & ECN618396 \\
\hline & NA & OSD-T-151-00007 7.2.8.a & $\begin{array}{l}\text { TO-001-182 } \\
\text { TO-001-184 } \\
\text { TO-040-760 }\end{array}$ & MA & \\
\hline
\end{tabular}

\section{5/31/96}

NA $=$ Not applicable or not required 
TABLE 6

Tank Farm or Miscellaneous Facility

AP

OSR/OSD SAFETY CONTROLS

\begin{tabular}{|c|c|c|c|c|c|}
\hline System/Structures/Components & OSR Limit & OSD Limit & Implementing Procedures & Verification & Comments \\
\hline & NA & OSD-T-151-00007 7.2.6.a & $\begin{array}{l}\text { TO-200-010 } \\
\text { TO-220-095 } \\
\text { TO-230-095 } \\
\text { TO-230-133 } \\
\text { TO-230-274 } \\
\text { TO-270-040 } \\
\text { TO-270-204 } \\
\text { TO-270-221 } \\
\text { TO-270-236 } \\
\text { TO-270-252 } \\
\text { TO-270-268 } \\
\text { TO-270-826 } \\
\text { TO-270-828 } \\
\text { TO-400-200 } \\
\text { TO-430-480 } \\
\text { TO-600-080 }\end{array}$ & NA & \\
\hline & NA & OSD-T-151-00007 7.2.6.b & $\begin{array}{l}\text { TO-001-182 } \\
\text { TO-001-184 } \\
\text { TO-040-760 } \\
\text { TO-200-010 } \\
\text { TO-220-095 } \\
\text { TO-230-095 } \\
\text { TO-230-133 } \\
\text { TO-230-274 } \\
\text { TO-270-040 } \\
\text { TO-270-204 } \\
\text { TO-270-221 } \\
\text { TO-270-236 } \\
\text { TO-270-252 } \\
\text { TO-270-266 } \\
\text { TO-270-826 } \\
\text { TO-270-828 } \\
\text { TO-400-200 } \\
\text { TO-480-480 } \\
\text { TO-600-080 }\end{array}$ & NA & \\
\hline & NA & OSD-T-151-00007 7.2.6.c & $\begin{array}{l}\text { TO-001-182 } \\
\text { TO-001-184 } \\
\text { TO-040-760 }\end{array}$ & $\mathbf{M A}$ & \\
\hline
\end{tabular}

$05 / 31 / 96$

NA $=$ Not applicable or not required

B-95 
TABLE 6

Tank Farm or Miscellaneous Facility

AP

OSR/OSD SAFETY CONTROLS

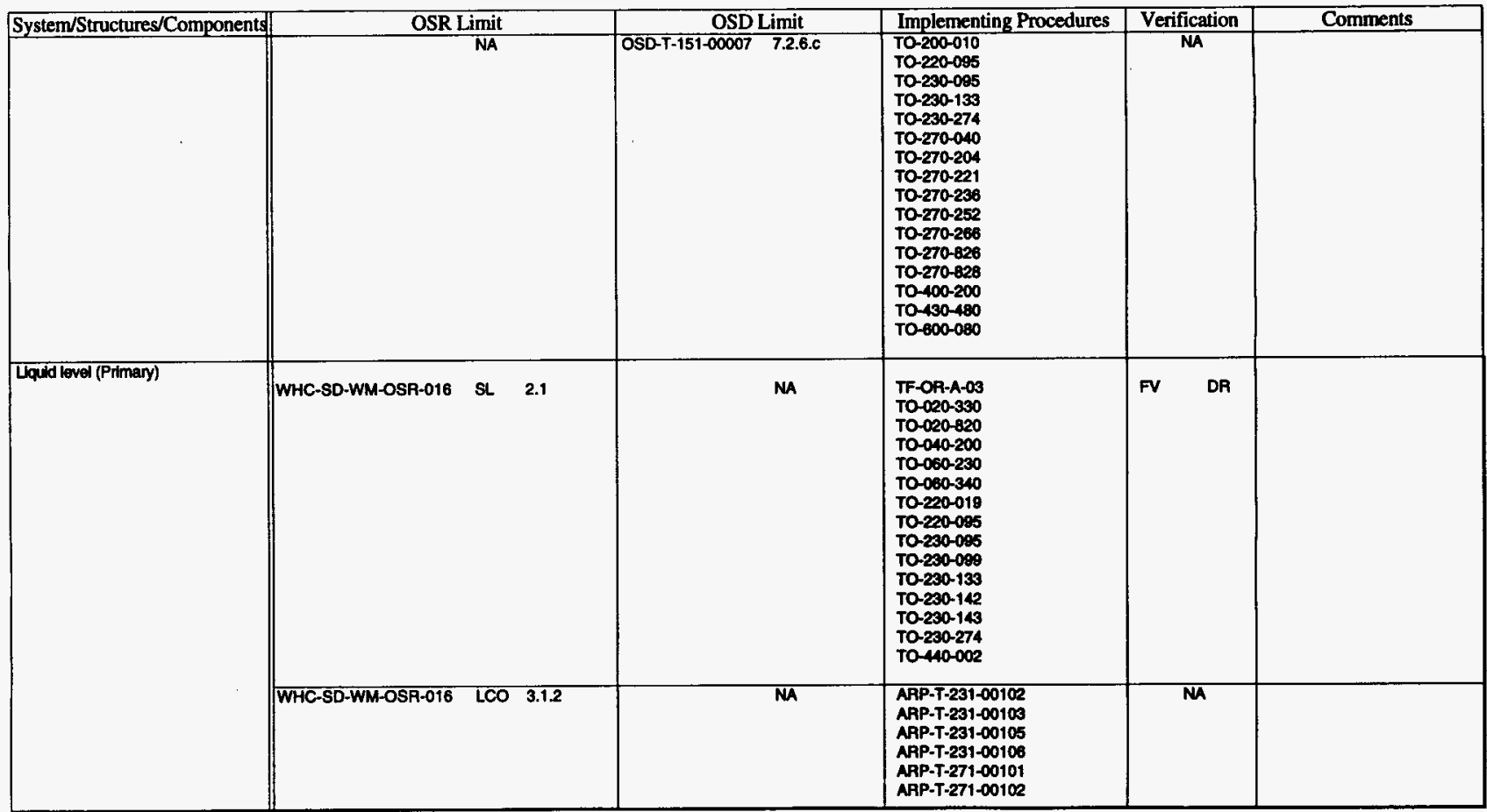

05/31/96

NA $=$ Not applicable or not required 
TABLE 6

Tank Farm or Miscellaneous Facility AP

OSR/OSD SAFETY CONTROLS

\begin{tabular}{|c|c|c|c|c|c|}
\hline System/Structures/Components & OSR Limit & OSD Limit & Implementing Procedures & Verification & Comments \\
\hline S & WHC-SD-WM-OSA-016 LCO 3.1 .2 & NA & $\begin{array}{l}\text { ARP.T-271-00103 } \\
\text { ARP-T-271-00104 } \\
\text { ARP-T-271-00105 } \\
\text { ARP-T-271-00106 } \\
\text { ARP-T-271-00107 } \\
\text { ARP-T-271-00106 } \\
\text { TF-OR-A-03 } \\
\text { TO-001-084 } \\
\text { TO-001-180 } \\
\text { TO-001-184 } \\
\text { TO-020-025 } \\
\text { TO-020-330 } \\
\text { TO-020-595 } \\
\text { TO-020-820 } \\
\text { TO-040-200 } \\
\text { TO-060-105 } \\
\text { TO-060-230 } \\
\text { TO-060-340 } \\
\text { TO-200-460 } \\
\text { TO-220-027 } \\
\text { TO-220-095 } \\
\text { TO-220-096 } \\
\text { TO-230-095 } \\
\text { TO-230-099 } \\
\text { TO-230-133 } \\
\text { TO-230-142 } \\
\text { TO-230-143 } \\
\text { TO-230-144 } \\
\text { TO-230-274 } \\
\text { TO-270-007 } \\
\text { TO-270-012 } \\
\text { TO-270-014 } \\
\text { TO-270-235 } \\
\text { TO-270-251 } \\
\text { TO-270-252 } \\
\text { TO-270-824 } \\
\text { TO-400-200 } \\
\text { TO-430-480 } \\
\text { TO-430-485 }\end{array}$ & NA & \\
\hline
\end{tabular}

05/31/96

NA $=$ Not applicable or not required 
TABLE 6

Tank Farm or Miscellaneous Facility AP

OSR/OSD SAFETY CONTROLS

\begin{tabular}{|c|c|c|c|c|c|c|c|}
\hline \multirow{3}{*}{ System/Structures/Components } & \multicolumn{3}{|c|}{ OSR Limit } & \multirow{2}{*}{$\frac{\text { OSD Limit }}{\mathrm{NA}}$} & \multirow{2}{*}{$\begin{array}{l}\text { Implementing Procedures } \\
\text { T0-440-002 }\end{array}$} & \multirow{2}{*}{$\frac{\text { Verification }}{\text { NA }}$} & \multirow[t]{2}{*}{ Comments } \\
\hline & WHC-SD-WM-OSR-016 & LCO & $\overline{3.1 .2}$ & & & & \\
\hline & WHC-SD-WM-SAR-016 & & 11.5 & OSD-T-151-00007 72.2.a & $\begin{array}{l}\text { TF-OA-A-03 } \\
\text { TO-001-182 } \\
\text { TO-020-240 } \\
\text { TO-020-420 } \\
\text { TO-020-820 } \\
\text { TO-040-590 } \\
\text { TO-230-095 } \\
\text { TO-230-274 } \\
\text { TO-270-204 } \\
\text { TO-270-221 } \\
\text { TO-270-236 } \\
\text { TO-270-252 } \\
\text { TO-270-266 } \\
\text { TO-270-826 } \\
\text { TO-270-828 }\end{array}$ & NA & \\
\hline Lquild lovel (Backup) & WHC-SD-WM-SAR-016 & & 11.5 & OSD-T-151-00007 7.2.2.a & $\begin{array}{l}\text { TF-OA-A-03 } \\
\text { TO-001-182 } \\
\text { TO-COO-240 } \\
\text { TO-020-420 } \\
\text { TO-020-820 } \\
\text { TO-040-590 } \\
\text { TO-290-095 } \\
\text { TO-230-274 } \\
\text { TO-270-204 } \\
\text { TO-270-221 } \\
\text { TO-270-236 } \\
\text { TO-270-252 } \\
\text { TO-270-266 } \\
\text { TO-270-826 } \\
\text { TO-270-628 }\end{array}$ & NA & \\
\hline High level probe & WHC-SD-WM-OSR-016 & $\mathbf{S R}$ & 3.1 .2 .1 & NA & $\begin{array}{l}\text { TF-OR-A-03 } \\
\text { TO-001-084 } \\
\text { TO-001-180 } \\
\text { TO-001-184 }\end{array}$ & NA & \\
\hline
\end{tabular}

$05 / 31 / 96$

NA $=$ Not applicable or not required 
TABLE 6

Tank Farm or Miscellaneous Facility AP

\section{OSR/OSD SAFETY CONTROLS}

\begin{tabular}{|c|c|c|c|c|c|c|}
\hline \multirow{2}{*}{ System/Structures/Components } & \multicolumn{2}{|c|}{ OSR Limit } & OSD Limit & Implementing Procedures & Verification & Comments \\
\hline & WHC-SD-WM-OSR-016 & SR $\quad 3.1 .2 .1$ & NA & $\begin{array}{l}\text { TO-020-025 } \\
\text { TO-020-330 } \\
\text { TO-020-595 } \\
\text { TO-020-820 } \\
\text { TO-040-200 } \\
\text { TO-060-340 } \\
\text { TO-200-400 } \\
\text { TO-200-460 } \\
\text { TO-220-095 } \\
\text { TO-220-096 } \\
\text { TO-230-095 } \\
\text { TO-230-099 } \\
\text { TO-230-133 } \\
\text { TO-230-141 } \\
\text { TO-230-142 } \\
\text { TO-230-143 } \\
\text { TO-230-144 } \\
\text { TO-270-007 } \\
\text { TO-270-012 } \\
\text { TO-270-014 } \\
\text { TO-270-235 } \\
\text { TO-270-251 } \\
\text { TO-270-252 } \\
\text { TO-400-200 } \\
\text { TO-430-480 } \\
\text { TO-430-485 } \\
\text { TO-440-002 }\end{array}$ & NA & \\
\hline \multirow[t]{2}{*}{ Tenk primary diff. pressure monilor } & WHC-SD-WM-OSA-016 & SL $\quad 2.3$ & NA & $\begin{array}{l}\text { TF-FT-239-001 } \\
\text { TF-FT-270-001 } \\
\text { TO-040-035 } \\
\text { TO-080-101 } \\
\text { TO-080-230 } \\
\text { TO-060-340 } \\
\text { TO-230-095 } \\
\text { TO-230-133 } \\
\text { TO-230-142 }\end{array}$ & FV DR & \\
\hline & WHC-SD-WM-OSR-016 & $\begin{array}{ll}\text { SL } & 2.4\end{array}$ & NA & TF-FT-230-001 & FV DA & \\
\hline
\end{tabular}

05/31/96

NA $=$ Not applicable or not required 
TABLE 6

Tank Farm or Miscellaneous Facillity AP

OSR/OSD SAFETY CONTROLS

\begin{tabular}{|c|c|c|c|c|c|}
\hline System/Structures/Components & OSR Limit & OSD Limit & Implementing Procedures & Verification & Comments \\
\hline \multirow[t]{2}{*}{ 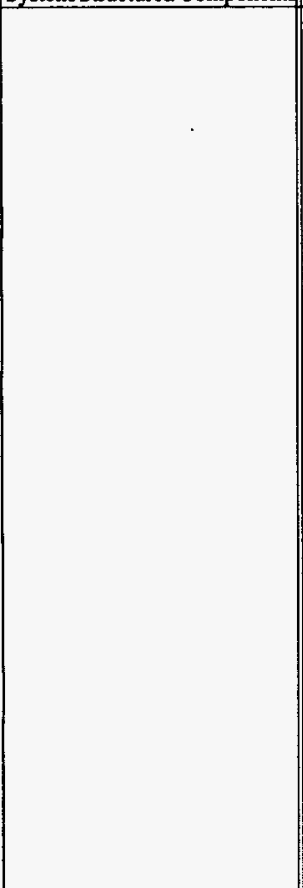 } & WHC-SD-WM-OSR-016 SL 2.4 & NA & $\begin{array}{l}\text { TF-FT-279-001 } \\
\text { TF-OA-A-03 } \\
\text { TO-040-035 } \\
\text { TO-060-101 } \\
\text { TO-060-230 } \\
\text { TO-060-340 } \\
\text { TO-230-095 } \\
\text { TO-230-095 } \\
\text { TO-230-133 } \\
\text { TO-230-142 }\end{array}$ & FV DR & \\
\hline & WHC-SD-WM-OSR-016 LCO 3.3 .1 & NA & $\begin{array}{l}\text { ARP-T-231-00102 } \\
\text { ARP-T-231-00103 } \\
\text { ARP-T-231-00104 } \\
\text { ARP-T-231-00105 } \\
\text { ARP-T-231-00106 } \\
\text { ARP-T-271-00101 } \\
\text { ARP-T-271-00102 } \\
\text { ARP-T-271-00104 } \\
\text { ARP-T-271-00105 } \\
\text { ARP-T-271-00106 } \\
\text { ARP-T-271-00107 } \\
\text { ARP-T-271-00108 } \\
\text { TF-FT-239-001 } \\
\text { TF-FT-279-001 } \\
\text { TO-001-084 } \\
\text { TO-001-184 } \\
\text { TO-040-035 } \\
\text { TO-060-101 } \\
\text { TO-060-230 } \\
\text { TO-060-340 } \\
\text { TO-200-019 } \\
\text { TO-200-464 } \\
\text { TO-220-027 } \\
\text { TO-220-095 } \\
\text { TO-230-095 } \\
\text { TO-230-099 } \\
\text { TO-230-133 } \\
\text { TO-230-141 } \\
\text { TO-230-142 }\end{array}$ & FV DA & \\
\hline
\end{tabular}

$05 / 31 / 96$

NA $=$ Not applicable or not required 
TABLE 6

Tank Farm or Miscellaneous Facility AP

\section{OSR/OSD SAFETY CONTROLS}

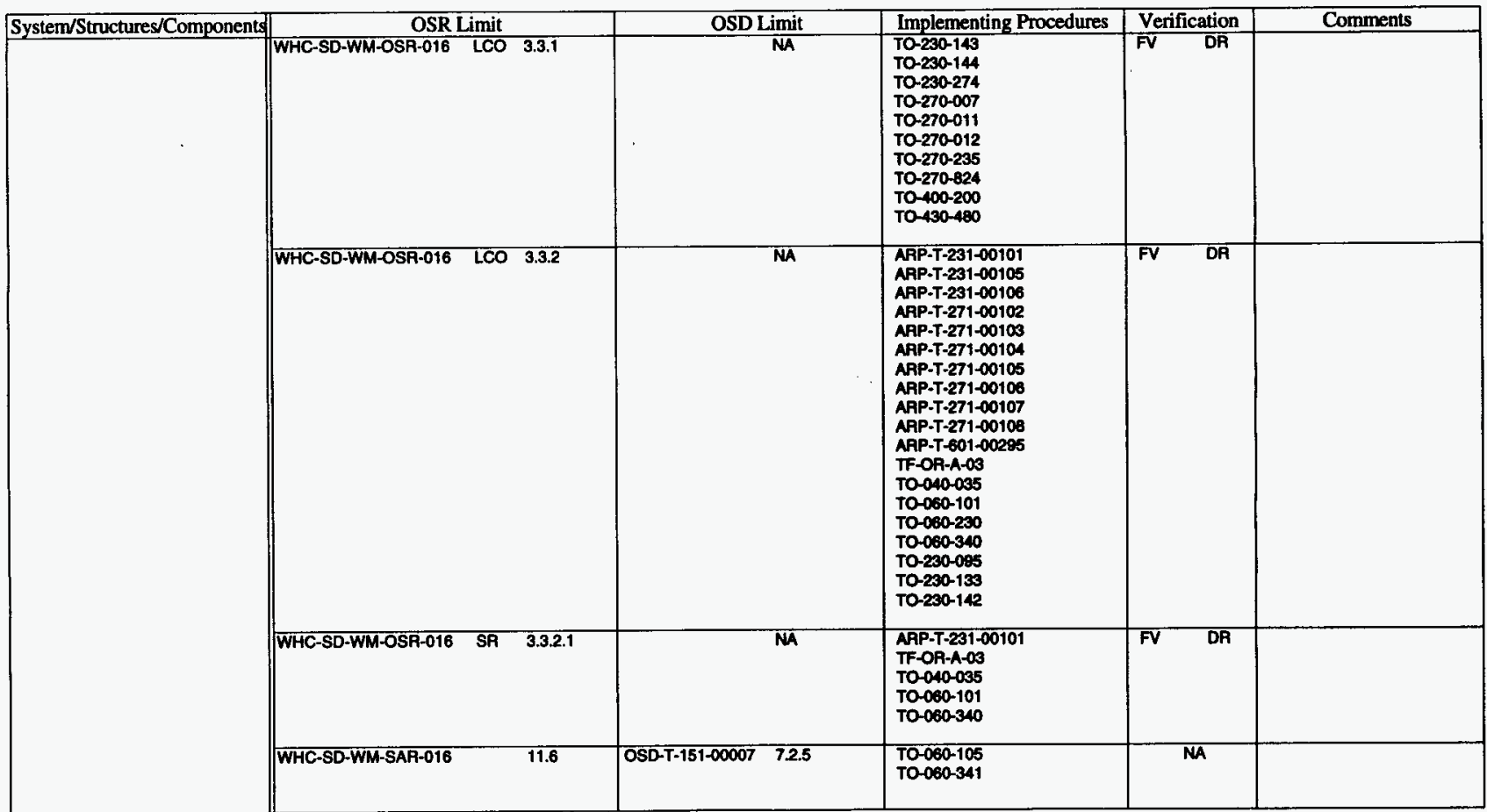


TABLE 6

Tank Farm or Miscellaneous Facility

AP

OSROSD SAFETY CONTROLS

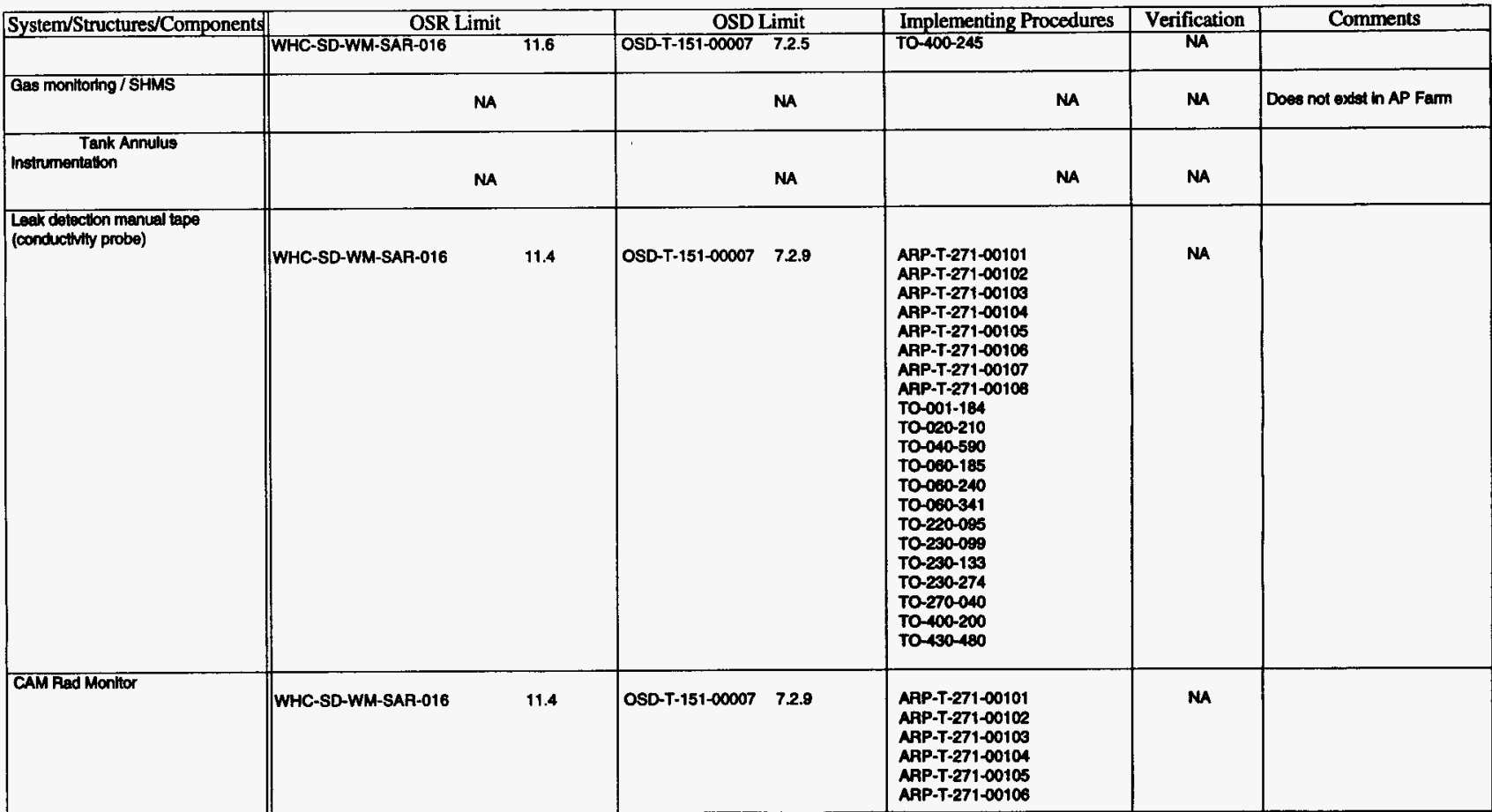

\section{$05 / 31 / 96$}

$\mathrm{NA}=$ Not applicable or not required 
TABLE 6

Tank Farm or Miscellaneous Facility AP

OSR/OSD SAFETY CONTROLS

\begin{tabular}{|c|c|c|c|c|c|c|c|c|}
\hline \multirow{2}{*}{ System/Structures/Components } & \multicolumn{3}{|c|}{ OSR Limit } & \multicolumn{2}{|c|}{ OSD Limit } & Implementing Procedures & \multirow{2}{*}{$\frac{\text { Verification }}{\mathrm{NA}}$} & \multirow[t]{2}{*}{ Comments } \\
\hline & WHC-SD-WM-SAR-016 & & 11.4 & OSD-T-151-00007 & 7.2 .9 & $\begin{array}{l}\text { ARP-T-271-00107 } \\
\text { ARP-T-271-00108 } \\
\text { TO-001-184 } \\
\text { TO-020-210 } \\
\text { TO-040-590 } \\
\text { TO-080-185 } \\
\text { TO-060-240 } \\
\text { TO-060-341 } \\
\text { TO-220-095 } \\
\text { TO-230-099 } \\
\text { TO-230-133 } \\
\text { TO-230-274 } \\
\text { TO-270-040 } \\
\text { TO-400-200 } \\
\text { TO-430-480 }\end{array}$ & & \\
\hline Pump pit leak detection & |WHC-SD-WM-SAR-016 & & 11.7 & OSD-T-151-00007 & 7.2 .10 & $\begin{array}{l}\text { TO-001-184 } \\
\text { TO-220-095 } \\
\text { TO-230-099 } \\
\text { TO-230-133 } \\
\text { TO-230-274 } \\
\text { TO-270-252 } \\
\text { TO-400-200 } \\
\text { TO-430-460 } \\
\text { TO-450-150 }\end{array}$ & MA & \\
\hline Temperature & & NA & & & NA & NA & NA & \\
\hline Tank annulus cifif. pressure monltor & & NA & & & NA & NA & NA & \\
\hline $\begin{array}{l}\text { Tank Structure } \\
\text { Instrumentation }\end{array}$ & & NA & & & NA & NA & NA & \\
\hline Thermocouple data acquisition & WHC-SD-WM-OSR-016 & $A C$ & 5.28 .1 & OSD-T-151-00007 & 7.2 .7 & $\begin{array}{l}\text { TF-OA-A-03 } \\
\text { TO-270-040 }\end{array}$ & NA & AC- Not Implemented by ECN \\
\hline
\end{tabular}

$05 / 31 / 96$

NA $=$ Not applicable or not required 
TABLE 6

Tank Farm or Miscellaneous Facility

AP

OSROSD SAFETY CONTROLS

\begin{tabular}{|c|c|c|c|c|c|}
\hline System/Structures/Components & OSR Limit & OSD Limit & Implementing Procedures & Verification & Comments \\
\hline & $\begin{array}{|lll|}\text { WHC-SD-WM-OSA-016 } & \text { AC } & 5.28 .1 \\
\end{array}$ & OSD-T-151-00007 7.2 .7 & T0-600-080 & NA & \\
\hline Instrument Enclosures & NA & NA & NA & NA & \\
\hline Ar lift chreulators & NA & NA & $\mathbf{M}$ & NA & Does not exdst in AP Farm \\
\hline Staem coll & NA & NA & NA & NA & Does not exdet I $\mathrm{h}$ AP Farm \\
\hline Calsson & NA & NA & NA & NA & Does not extst in AP Fam \\
\hline HVAC Systems & NA & NA & NA & NA & \\
\hline Ventilation System(s) & NA & NA & NA & NA & \\
\hline Passtvo & NA & NA & NA & NA & \\
\hline Active & NA & OSD-T-151-00007 $\quad 7.3 .2$ & 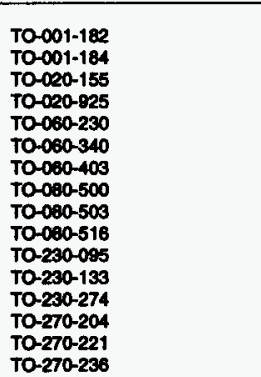 & NA & \\
\hline
\end{tabular}

$05 / 31 / 96$

NA $=$ Not applicable or not required

B-104 
TABLE 6

Tank Farm or Miscellaneous Facility

AP

OSR/OSD SAFETY CONTROLS

\begin{tabular}{|c|c|c|c|c|c|c|c|}
\hline \multirow[t]{2}{*}{ System/Structures/Components } & \multicolumn{2}{|c|}{ OSR Limit } & \multicolumn{2}{|c|}{ OSD Limit } & \multirow{2}{*}{$\begin{array}{l}\text { Implementing Procedures } \\
\text { TO-270-252 } \\
\text { TO-270-266 } \\
\text { TO-270-826 } \\
\text { TO-270-828 }\end{array}$} & \multirow{2}{*}{$\frac{\text { Verification }}{\text { NA }}$} & \multirow[t]{2}{*}{ Comments } \\
\hline & & NA & OSD-T-151-00007 & 7.3 .2 & & & \\
\hline $\begin{array}{l}\text { Shared / common vent system? } \\
\text { (Descitbe in comments section) }\end{array}$ & & NA & & NA & NA & NA & \\
\hline Exhaust venthatchway & & NA & & NA & NA & NA & \\
\hline Annutus ali intake assemblles & & NA & & NA & NA & NA & \\
\hline Primary air htake assemblles & & NA & & NA & NA & Na & Does not exist in AP Farm \\
\hline Pro-filters (yes/no) & & NA & & NA & NA & MA & \\
\hline $\begin{array}{l}\text { HEPA filtirs (how many fitters, } \\
\text { stages, parallet v3 serles etc.) }\end{array}$ & & NA & & NA & NA & MA & \\
\hline Fans & & MA & & NA & NA & Ma & \\
\hline De-ntrainers & & NA & & NA & NA & $\mathbf{M a}$ & \\
\hline Healors & & NA & & MA & NA & MA & \\
\hline HVAC Instrumentation & WHC-SD-WM-OSA-016 & LCO $\quad 3.4 .1$ & OSD-T-151-00007 & 7.3.1.A & $\begin{array}{l}\text { TO-060-101 } \\
\text { TO-060-104 } \\
\text { TO-060-105 } \\
\text { TO-060-230 }\end{array}$ & NA & $\begin{array}{l}\text { LCO-Not implemented by } \\
\text { ECN }\end{array}$ \\
\hline
\end{tabular}

05/31/96

NA $=$ Not applicable or not required 
WHC-SD-WM-HIE-007 REV 0

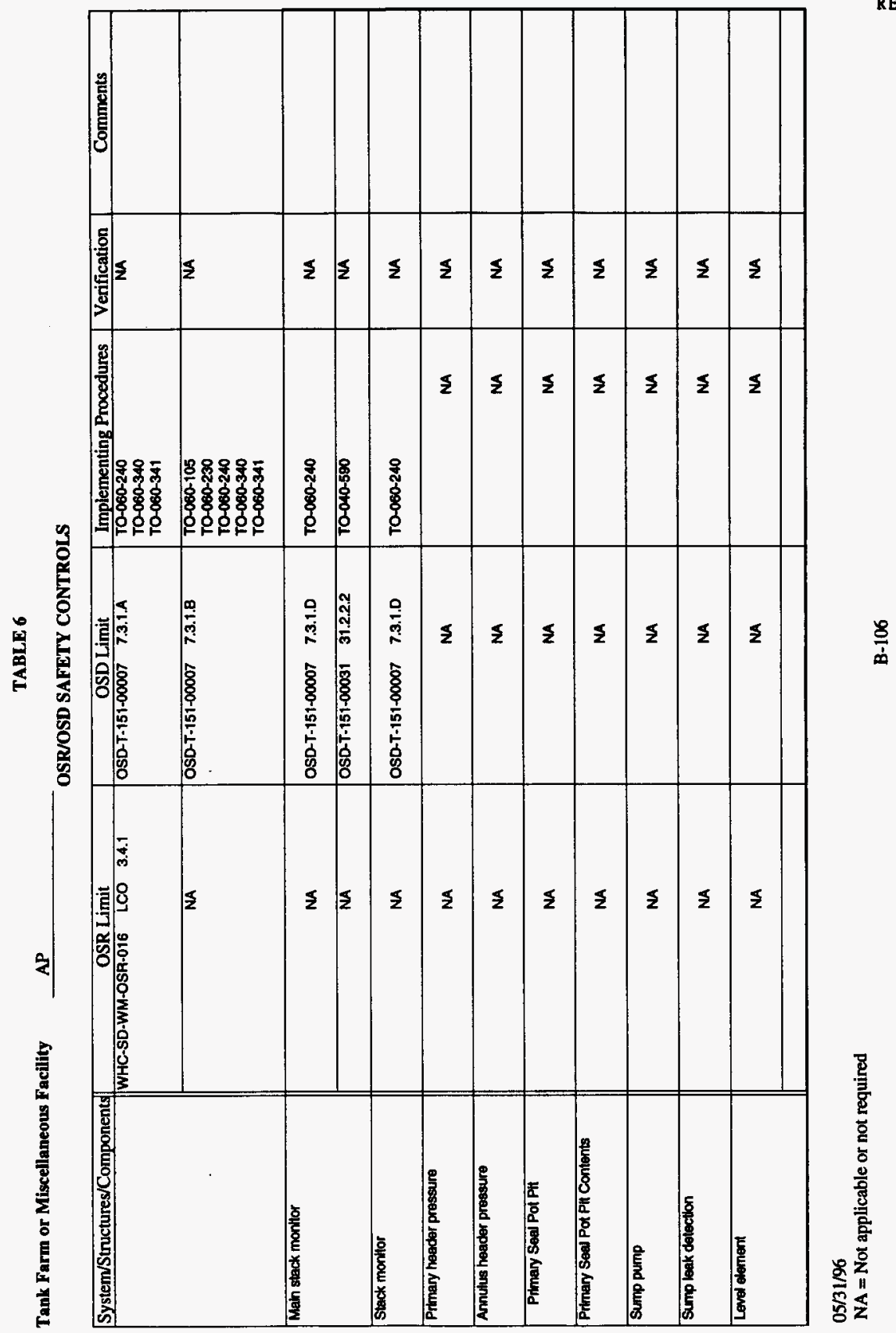


TABLE 6

Tank Farm or Miscellaneous Facility

AP

OSROSD SAFETY CONTROLS

\begin{tabular}{|c|c|c|c|c|c|}
\hline System/Structures/Components & OSR Limit & OSD Limit & Implementing Procedures & Verification & Comments \\
\hline Drain & NA & NA & MA & NA & \\
\hline Heat trace & NA & MA & NA & NA & \\
\hline Annulus Seat Pot Plt & NA & NA & NA & NA & \\
\hline Annulus Seal Pot Plt Contents & NA & NA & NA & MA & \\
\hline Level element & NA & NA & NA & MA & \\
\hline Draln & NA & NA & NA & NA & \\
\hline Ventilation Instrument PAt & NA & MA & NA & NA & \\
\hline Venwlation Instrument Prt contents & NA & NA & MA & MA & \\
\hline TBD & NA & NA & M & NA & \\
\hline Pilis & NA & MA & NA & M & \\
\hline Annitus Pump Pit & NA & NA & NA & M & \\
\hline Annulus Pump Pit Contents & NA & NA & MA & NA & \\
\hline Foor drain & NA & MA & NA & NA & \\
\hline
\end{tabular}

05/31/96

NA $=$ Not applicable or not required 
WHC-SD-WM-HIE-007

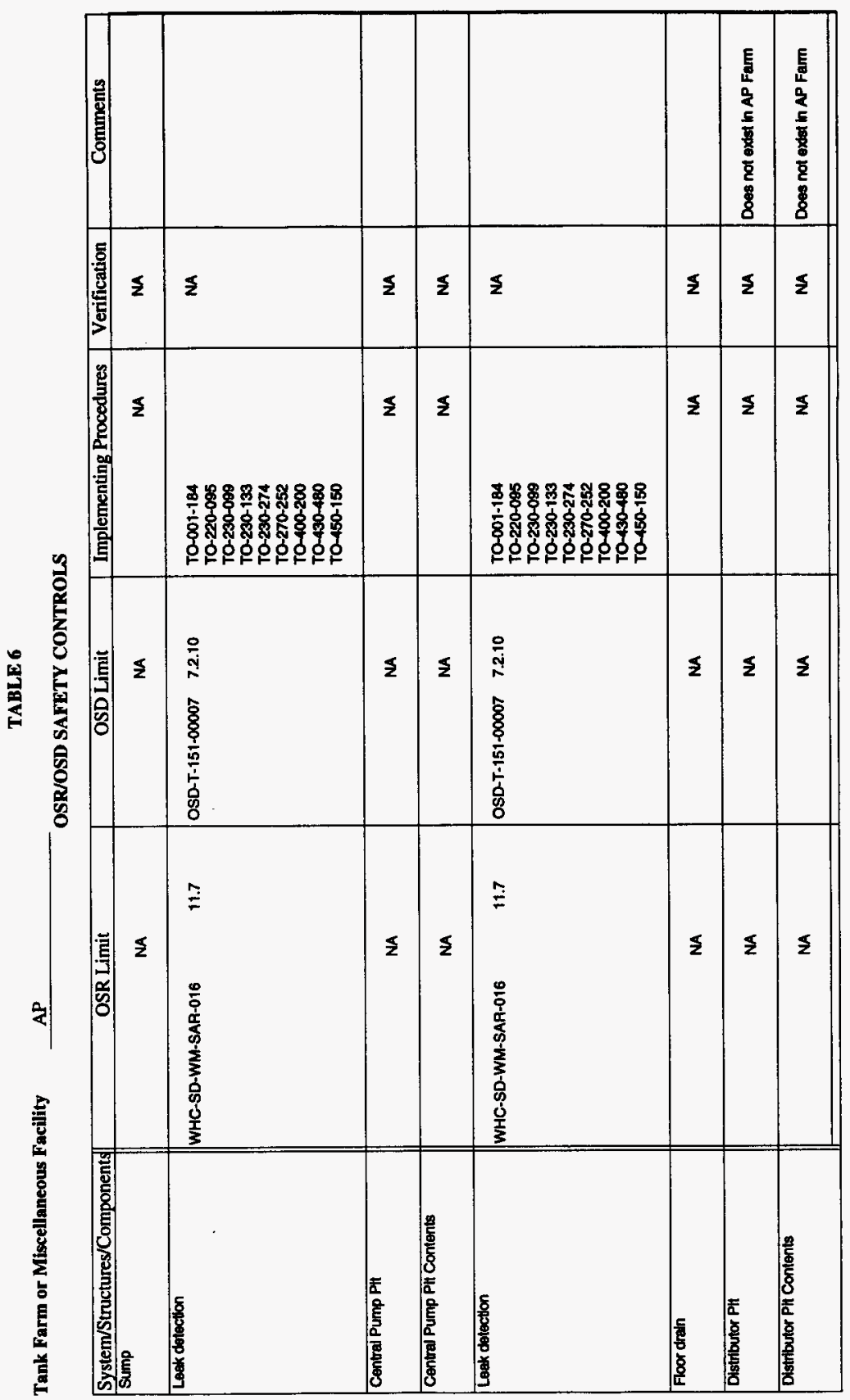

REV 0 


\begin{tabular}{|c|c|c|c|c|c|}
\hline & WN & $\mathbf{w}$ & $\mathbf{m}$ & $\mathbf{V N}$ & LOAnow pes Buruerg \\
\hline & W & 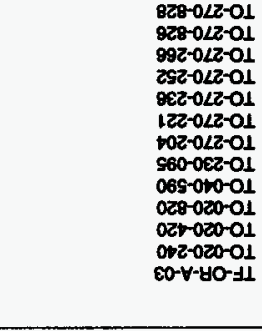 & Q'ZZL LO000-ISI-L-OSO & $\mathbf{W N}$ & 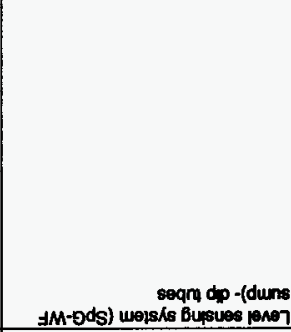 \\
\hline & $\mathbf{W}$ & $\mathbf{W}$ & W & VN & $\begin{array}{l}\text { OSO PU: OSO - Uopquounnsu } \\
\text { dunsMld UopeleO }\end{array}$ \\
\hline & $\mathbf{w}$ & WN & $w$ & $\mathbf{V N}$ & yd dund uopoepep yEeר \\
\hline une d dV u pepto pou seco & WN & VN & W & $\mathbf{V N}$ & छuequoO Hd 10ен \\
\hline Wred dY U kepe fou seod & $\mathbf{W}$ & VN & VN & $\mathbf{V N}$ & Hd $100 \mathrm{H}$ \\
\hline Urey dV U ippe pou 0000 & $w$ & $\mathbf{W}$ & WN & $\mathbf{V N}$ & छuथquOO Hd \\
\hline Uvey dV WI ISpX0 10u 0000 & $\mathbf{w}$ & $\mathbf{W}$ & vo & $\mathbf{V N}$ & Hd exins \\
\hline squatumoD & uop̣esỵ!a & 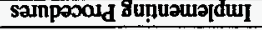 & !!ш!' GSO & 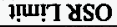 & 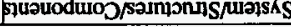 \\
\hline
\end{tabular}


System/Structures/Components

\begin{tabular}{|l||}
\hline Leak detection system \\
\hline Level dement \\
\hline Temperature \\
\hline Sump \\
\hline Drahn \\
\hline Dry wells \\
\hline Condenser pit \\
\hline Condenser Pth Contents \\
\hline Studge pump pit \\
\hline Sludge pump pit conlents \\
\hline Salt well pump pit \\
\hline Saltwell Pumping instrumentation \\
\hline Hydrogen monitor \\
\hline
\end{tabular}

$05 / 31 / 96$

NA $=$ Not applicable or not required

\section{OSR/OSD SAFETY CONTROLS}

\begin{tabular}{|c|c|c|c|c|}
\hline OSR Limit & OSD Limit & Implementing Procedures & Verification & Comments \\
\hline NA & NA & NA & MA & \\
\hline NA & NA & NA & NA & \\
\hline NA & NA & NA & NA & \\
\hline NA & NA & $\mathbf{N A}$ & NA & \\
\hline NA & NA & NA & NA & \\
\hline NA & NA & NA & NA & \\
\hline NA & NA & NA & NA & Does not exlst in AP Farm \\
\hline NA & NA & NA & NA & Does not exdat in AP Farm \\
\hline NA & NA & $\mathbf{N A}$ & NA & Does not exdst in AP Fam \\
\hline NA & NA & NA & NA & Does not exiat in AP Fam \\
\hline NA & NA & NA & NA & Does not extst in AP Fam \\
\hline NA & NA & NA & NA & Does not exdst in AP Fam \\
\hline NA & NA & NA & NA & Does not exdst in AP Farm \\
\hline & & & & \\
\hline
\end{tabular}

B-110 
TABLE 6

Tank Farm or Miscellaneous Facility

AP

OSR/OSD SAFETY CONTROLS

\begin{tabular}{|c|c|c|c|c|c|c|c|}
\hline \multirow{2}{*}{\begin{tabular}{|l|} 
System/Structures/Components \\
Welght factor / specific gravity
\end{tabular}} & \multicolumn{2}{|c|}{ OSR Limit } & \multicolumn{2}{|c|}{ OSD Limit } & Implementing Procedures & \multirow{2}{*}{$\frac{\text { Verification }}{\text { NA }}$} & \multirow{2}{*}{\begin{tabular}{|c|} 
Comments \\
Does not extst in AP Farm
\end{tabular}} \\
\hline & & & & NA & NA & & \\
\hline Drain pit & & & & NA & NA & NA & \\
\hline Draln Pit Contents & & & & MA & NA & NA & \\
\hline Draln & & & & NA & NA & NA & \\
\hline Loek dotection & WHC-SD-WM-SAR-016 & 11.7 & OSD-T-151-00007 & 7.2.10 & $\begin{array}{l}\text { TO-001-184 } \\
\text { TO-220-095 } \\
\text { TO-230-099 } \\
\text { TO-230-133 } \\
\text { TO-230-274 } \\
\text { TO-270-252 } \\
\text { TO-400-200 } \\
\text { TO-430-480 } \\
\text { TO-450-150 }\end{array}$ & MA & \\
\hline $\begin{array}{l}\text { Valve PHi / Transier PHt / Dlversion } \\
\text { Box }\end{array}$ & & & & NA & NA & NA & \\
\hline $\begin{array}{l}\text { Valve PrtTranster ptV Otversion Box } \\
\text { contents }\end{array}$ & & & & MA & NA & NA & \\
\hline Loek detection & WHC-SD-WM-SAR-016 & 11.7 & OSD-T-151-00007 & 7.2 .10 & $\begin{array}{l}\text { TO-001-184 } \\
\text { TO-220-095 } \\
\text { TO-230-099 } \\
\text { TO-230-133 } \\
\text { TO-230-274 } \\
\text { TO-270-252 } \\
\text { TO-400-200 } \\
\text { TO-430-480 }\end{array}$ & MA & \\
\hline
\end{tabular}

05/31/96

NA $=$ Not applicable or not required 
TABLE 6

Tank Farm or Miscellaneous Facility

AP

OSR/OSD SAFETY CONTROLS

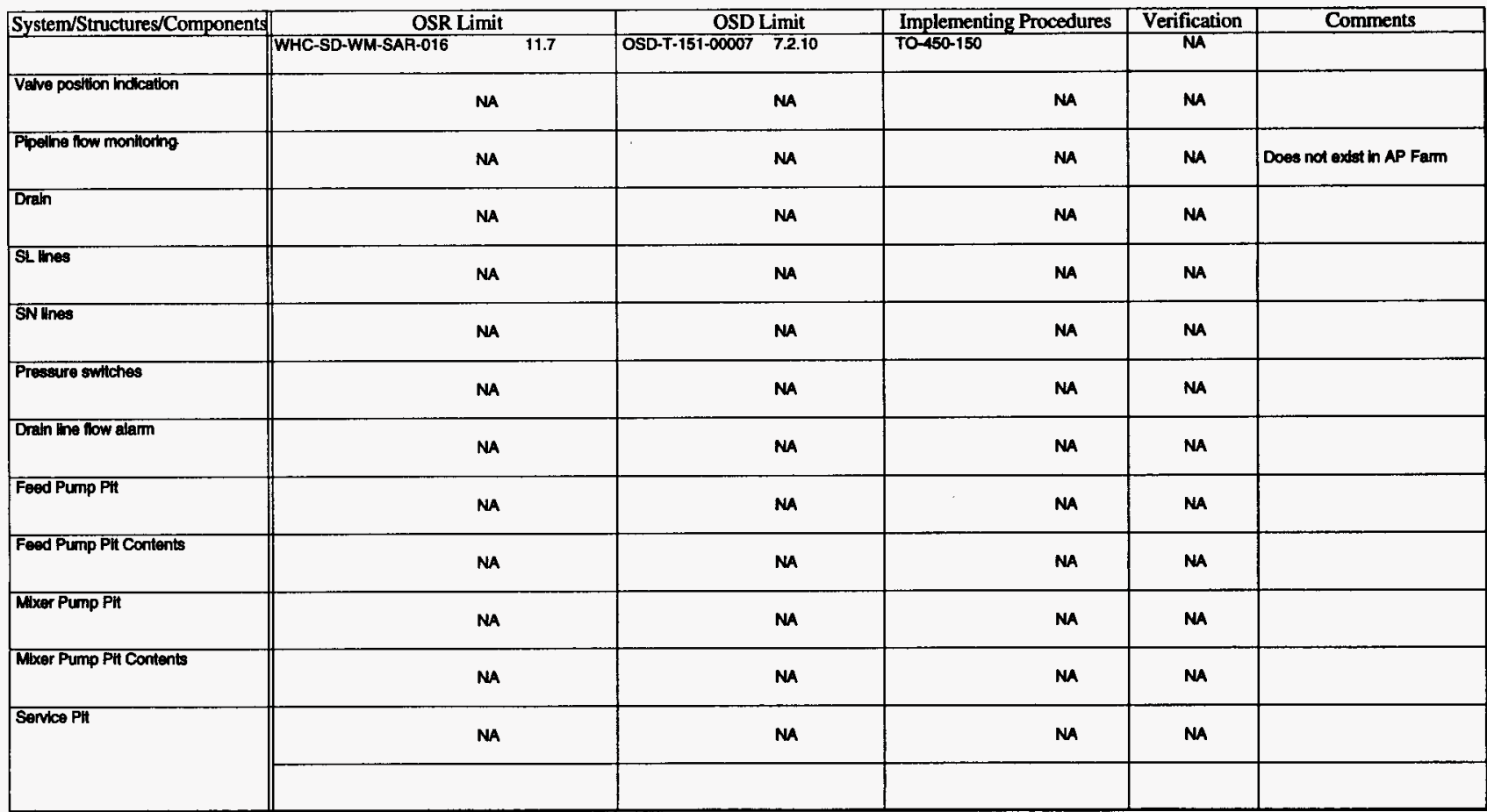

05/31/96

NA $=$ Not applicable or not required 
TABLE 6

Tank Farm or Miscellaneous Facility

AP

OSR/OSD SAFETY CONTROLS

\begin{tabular}{|c|c|c|c|c|c|}
\hline System/Structures/Components & OSR Limit & OSD Limit & Implementing Procedures & Verification & Comments \\
\hline Service plt hstrumentation & NA & NA & NA & NA & \\
\hline Flow monitoring & NA & NA & NA & NA & \\
\hline Rad monitior & NA & NA & NA & NA & \\
\hline Backiliow preventer & NA & NA & NA & NA & \\
\hline Leak detection & WHC-SD-WM-SAR-016 11.7 & OSD-T-151-00007 7.2 .10 & $\begin{array}{l}\text { TO-001-t84 } \\
\text { TO-220-095 } \\
\text { TO-230-099 } \\
\text { TO-230-133 } \\
\text { TO-230-274 } \\
\text { TO-270-252 } \\
\text { TO-400-200 } \\
\text { TO-430-480 } \\
\text { TO-450-150 }\end{array}$ & NA & \\
\hline Sump lovel element & NA & NA & NA & NA & \\
\hline Pressure Gauges & NA & NA & NA & NA & \\
\hline Sump & NA & NA & NA & NA & \\
\hline Drain & NA & NA & NA & NA & \\
\hline Heater & NA & NA & NA & NA & \\
\hline
\end{tabular}

$05 / 31 / 96$

NA $=$ Not applicable or not required 
TABLE 6

Tank Farm or Miscellaneous Facility

AP

OSR/OSD SAFETY CONTROLS

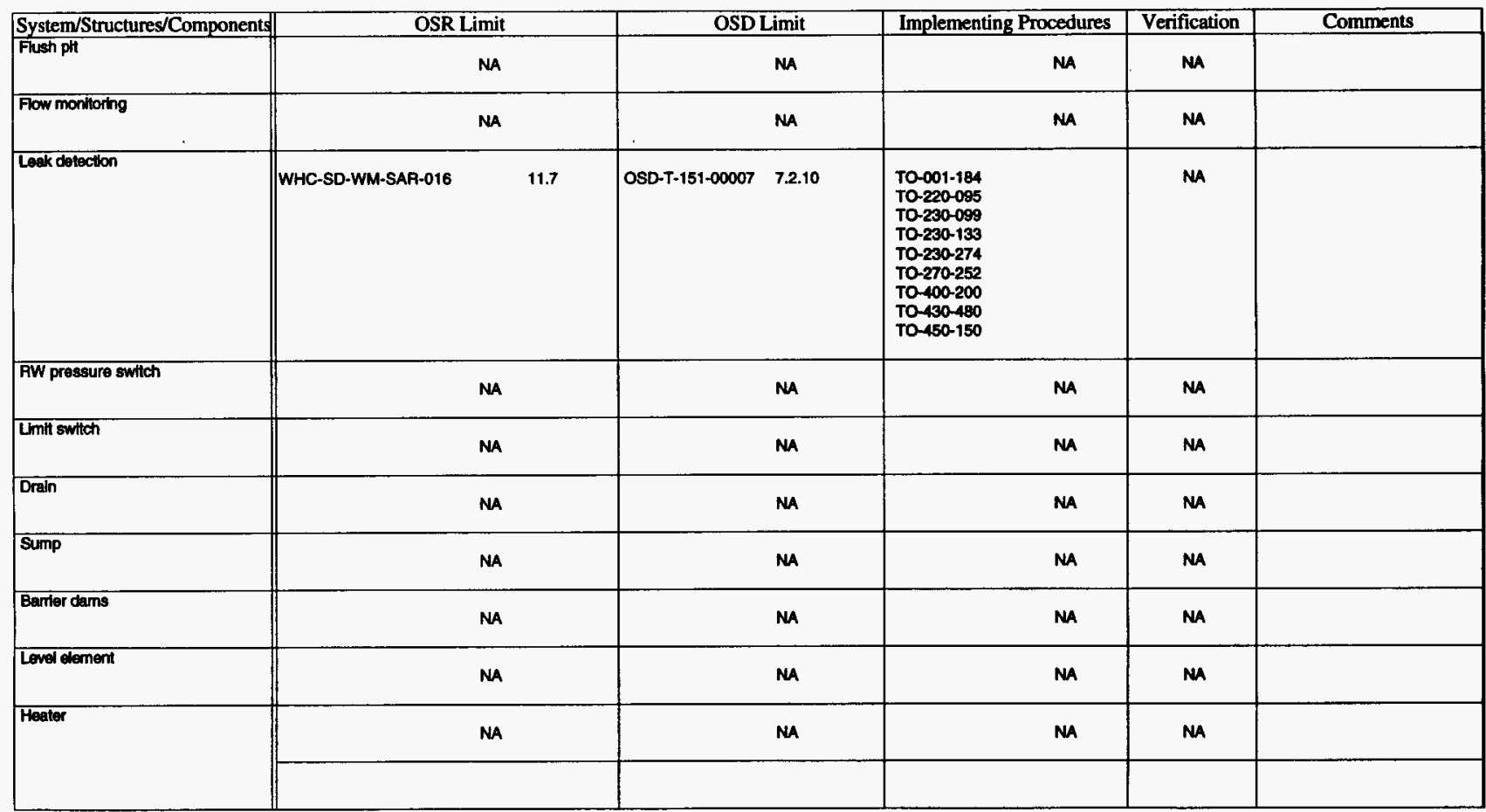

05/31/96

NA $=$ Not applicable or not required 


\begin{tabular}{|c|c|c|c|c|c|}
\hline System/Structures/Components & OSR Limit & OSD Limit & Implementing Procedures & Verification & Comments \\
\hline & NA & NA & NA & NA & \\
\hline Transter Piphng & NA & NA & NA & NA & \\
\hline Transfer Llnes & NA & NA & NA & NA & \\
\hline Bulldings & NA & NA & MA & NA & \\
\hline Compressor Bullding & Na & Ma & NA & NA & \\
\hline Holding Tanks & NA & NA & NA & NA & \\
\hline Flammable storage cabinet & NA & NA & NA & NA & \\
\hline Instrument Bullowing & NA & MA & MA & NA & \\
\hline $\begin{array}{l}\text { Adlustable Frequency AC Ditvo for } \\
\text { AP-102 Mitxer pump }\end{array}$ & NA & NA & NA & NA & \\
\hline $\begin{array}{l}\text { Instument Building } \\
\text { Inotrumentalion }\end{array}$ & NA & MA & MA & MA & \\
\hline CASS connection & NA & NA & NA & NA & \\
\hline CAM & NA & NA & NA & NA & \\
\hline & & & & & \\
\hline
\end{tabular}

\section{5/31/96}

NA $=$ Not applicable or not required 
TABLE 6

Tank Farm or Miscellaneous Facility

AP

OSR/OSD SAFETY CONTROLS

\begin{tabular}{|c|c|c|c|c|c|}
\hline System/Structures/Components & OSR Limit & OSD Limit & Implementing Procedures & Verification & Comments \\
\hline $\begin{array}{l}\text { Remote alam location(s) (describe } \\
\text { in comment section) }\end{array}$ & NA & NA & NA & NA & \\
\hline Heat trace & NA & NA & NA & MA & \\
\hline Steem & NA & NA & NA & NA & Does not exdet in AP Farm \\
\hline Steam traps & NA & NA & NA & NA & Does not exdst in AP Farm \\
\hline Miscelianeous Facilites & NA & NA & NA & NA & \\
\hline Electrical Substation & NA & NA & NA & NA & \\
\hline Motor Control Station & NA & NA & NA & NA & \\
\hline Botte Storage Area & NA & NA & NA & NA & \\
\hline Heat Trace Transtormer/Panel & NA & MA & NA & NA & \\
\hline Change Traller & NA & NA & NA & NA & \\
\hline
\end{tabular}

05/31/96

NA $=$ Not applicable or not required 


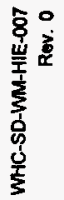

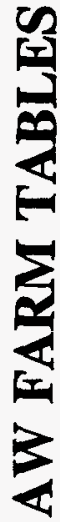

B-117 


\section{TWRS FSAR MATRIX TABLE VERIFICATION REVIEW APPROVALS \\ May 31, 1996}

The following information has been assembled, organized, and documented in the attached TWRS Final Safety Analysis Report (FSAR) Matrix Tables in support of Tank Farms FSAR development. Science Applications International Corporation (SAIC), along with the assistance of ICF Kaiser Hanford and the Westinghouse Hanford Company, assembled this data from controlled drawings and databases, supporting documents, as well as other site documentation. Where possible, the data has been field verified by performing field walkdowns of the particular facility of interest. The data also have been reviewed for its completeness and accuracy by the TWRS Facility Operations Design Authority and the Cognizant Engineer assigned to the respective facility. The information, to the best of the knowledge and the time allotted by the undersigned, is true, accurate, and complete as permitted by the referenced documents and drawings shown on the respective tables. No efforts were attempted or made by the authors and/or reviewers to substantiate the reference information shown on the tables beyond the stated qualifiers as noted on the individual facility matrix tables.

\section{AW Tank Farm}

Tank Farm or Facility
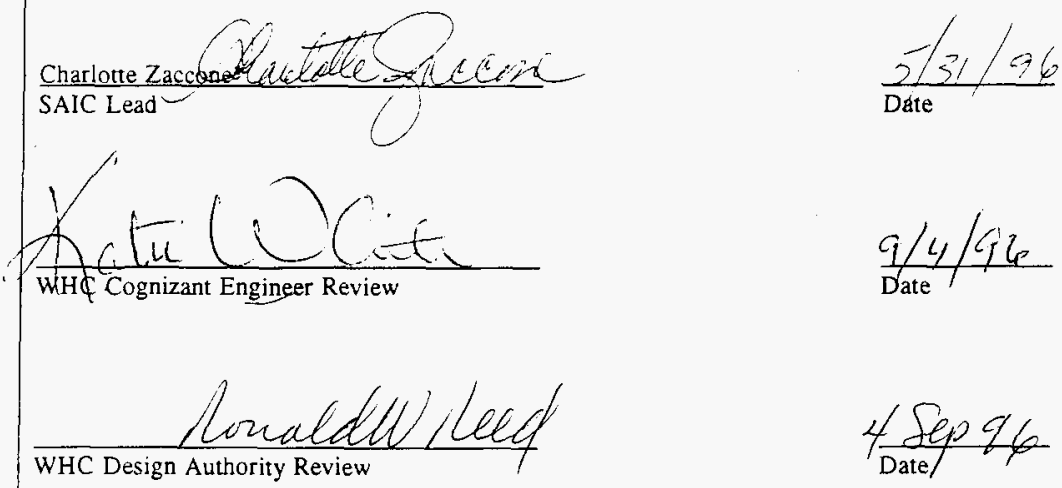

\footnotetext{
* Stone and Webster support
} 


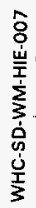

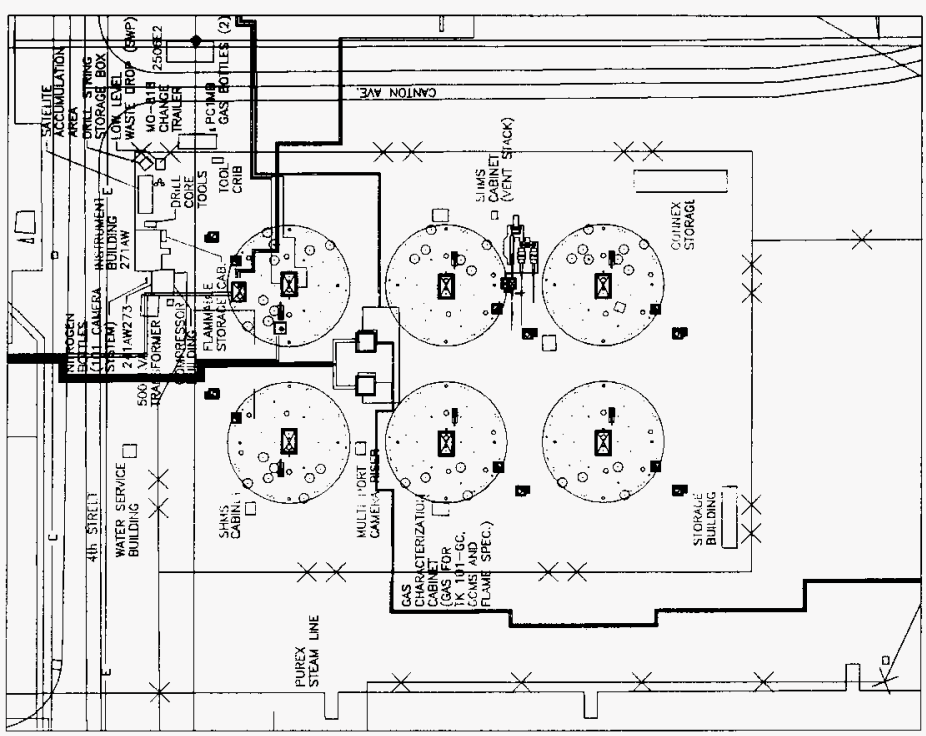

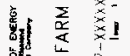

粼

造

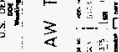

1]

Ent

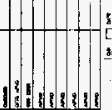

1
-1

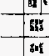

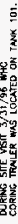
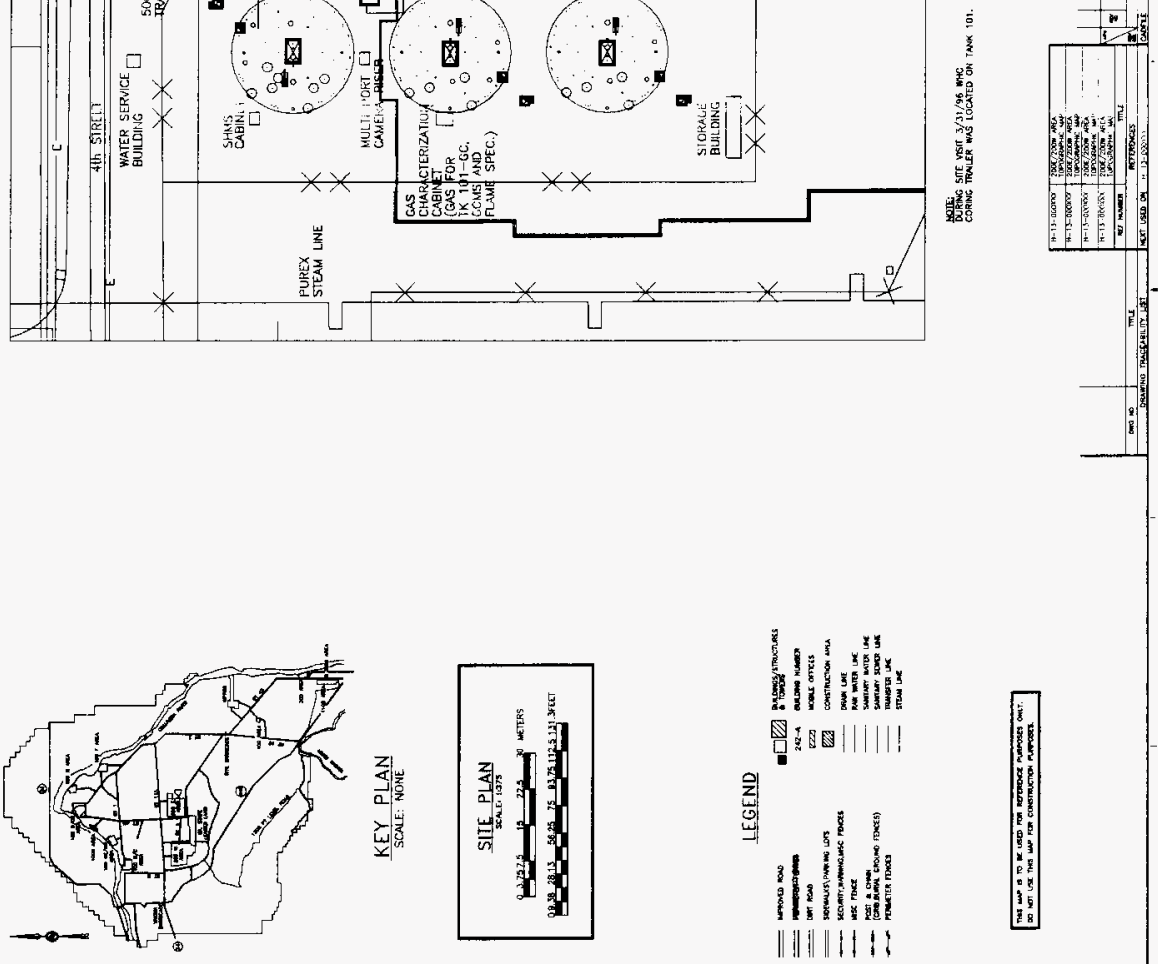
AWFARM

WHC. SD-WM- HIE-007

MASTER CHECAST

hov, 0

\begin{tabular}{|c|c|c|c|c|c|c|c|c|}
\hline Facility & Typo & $\begin{array}{l}\text { Mateid invertory, } \\
\text { (Tathe 1) }\end{array}$ & 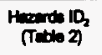 & $\begin{array}{l}\text { Uhiqu Fheroments } \\
\text { (Tible 3) }\end{array}$ & $\begin{array}{l}\text { Facily Contiartion } \\
\text { (Tribs } 4\end{array}$ & $\begin{array}{l}\text { Pad Lench } \\
\text { (Tato } 5)\end{array}$ & Contuth & Comment \\
\hline Hot & & & 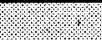 & & & & & \\
\hline 241AN-101 & DST & $x$ & $x$ & $x$ & $x$ & $x$ & $x$ & $M$ \\
\hline 241 A4 102 & DST & $x$ & $x$ & $x$ & $x$ & $x$ & $x$ & $\mathrm{Mn}$ \\
\hline 241-AWV 103 & DST & $x$ & $x$ & $x$ & $x$ & $x$ & $x$ & $m$ \\
\hline 241AN-104 & DST & $x$ & $x$ & $x$ & $x$ & $x$ & $x$ & $M$ \\
\hline 241AW-105 & DST & $x$ & $x$ & $x$ & $x$ & $x$ & $x$ & $\mathrm{M}$ \\
\hline 241-AN+106 & DST & $x$ & $x$ & $x$ & $x$ & $x$ & $x$ & $M$ \\
\hline COBAW01 & Clemoul box & $M$ & $m$ & $M$ & $M$ & MA & $m$ & $\begin{array}{l}\text { Euloros to } 242 \text { A } \\
\text { Exponior }\end{array}$ \\
\hline COBAWOR & Clemoul box & $\mathbf{M A}$ & $M$ & $M$ & $M$ & $\mathbf{M}$ & $M$ & $\begin{array}{l}\text { Betones it 242-A } \\
\text { Eveponitor }\end{array}$ \\
\hline OOBAN"OS & Clesnout box & MA & M & $M$ & $M$ & $M$ & $m$ & $\begin{array}{l}\text { Belones of 242A } \\
\text { Extpontor }\end{array}$ \\
\hline COBAMro4 & Comout box & $M$ & MA & $M$ & $m$ & $\mathbf{M}$ & $m$ & 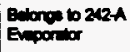 \\
\hline COBAWOS & Cleenout box & $M$ & $M$ & M & $M$ & $M$ & $M$ & $\begin{array}{l}\text { Belonge to } 262 A \\
\text { Exponter }\end{array}$ \\
\hline COBAW06 & Cleanoul box & $M$ & $M$ & $M$ & $M$ & $m$ & $M$ & 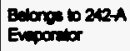 \\
\hline Cosanor & Cleanoul box & MA & $M$ & MA & MA & $M$ & $M$ & $\begin{array}{l}\text { Eulores io } 242 A \\
\text { Evepontor }\end{array}$ \\
\hline COBAWOS & Ceanoul bax & MA & $M$ & $M$ & $M$ & $M$ & $M$ & $\begin{array}{l}\text { Belonos to 242,A } \\
\text { Empontor }\end{array}$ \\
\hline
\end{tabular}

NA = Not applicable or not recuired

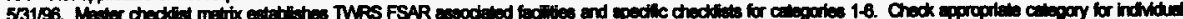

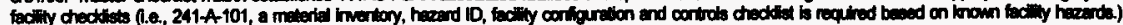

$$
\text { B-120 }
$$


AW FARM

WHC-SD.WM-HIE-0O7

MASTER CHECAST

Rov. 0

\begin{tabular}{|c|c|c|c|c|c|c|c|c|}
\hline Fecitity & Type & 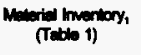 & Heards $x_{2}$ & $\begin{array}{c}\text { Uniquo Pronomere, } \\
\text { (Table 3) }\end{array}$ & 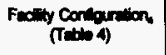 & Ped Lover & (Tontioles & Cormment \\
\hline Cosanios & Clemout bax & $x$ & $x$ & M & $x$ & $x$ & $x$ & M \\
\hline $\cos A N_{10}$ & Creanoul box & $x$ & $x$ & $M$ & $x$ & $x$ & $x$ & M \\
\hline COBAWI1 & Clement box & MA & $\mathbf{M}$ & $m$ & $\mathrm{M}$ & $\mathrm{M}$ & $M$ & $\begin{array}{l}\text { Belonge b 212A } \\
\text { Evponter }\end{array}$ \\
\hline COBAN12 & Cleanoul box & MA & $\mathrm{MA}$ & MA & $M$ & $M$ & M & Edonos b 212A \\
\hline $\cos A 30$ & Clesnoul box & M & MA & MA & M & M & $\mathrm{M}$ & $\begin{array}{l}\text { Belonos b 212A } \\
\text { Exporitar }\end{array}$ \\
\hline 241-AW701 & $\begin{array}{l}\text { Comprosedor } \\
\text { bulling }\end{array}$ & NA & $x$ & $m$ & $x$ & $x$ & $x$ & NA \\
\hline 241-AWLOEF & Feed pump pt & $x$ & $x$ & MA & $x$ & $x$ & $x$ & MA \\
\hline 241-AW & Fush pit & $x$ & $x$ & $M$ & $x$ & $x$ & $x$ & $\mathbf{M}$ \\
\hline 241-Aพ-271 & $\begin{array}{l}\text { moltument } \\
\text { building }\end{array}$ & MA & $x$ & $m$ & $x$ & $x$ & $x$ & $M$ \\
\hline 241-AW-272 & Operition: & $x$ & $x$ & M & $x$ & $M$ & $x$ & $M$ \\
\hline 241-AWE81 & Senvioe pit & $M$ & $M$ & M & M & MA & $M$ & $\operatorname{Sumb}_{A N 273}$ \\
\hline 241-AW273 & Senvios bulling & $x$ & $x$ & MA & $x$ & $x$ & $x$ & M \\
\hline 241AWA & Vowe pit & $x$ & $x$ & $M$ & $x$ & $x$ & $x$ & M \\
\hline \multirow[t]{2}{*}{ 241-AWB } & Vhe pit & $x$ & $x$ & $M$ & $x$ & $x$ & $x$ & $M$ \\
\hline & $\begin{array}{l}\text { Sactims } \\
\text { Acamition } \\
\text { Area }\end{array}$ & $x$ & $x$ & $M$ & $x$ & $x$ & $x$ & $M$ \\
\hline
\end{tabular}

NA $=$ Not epplicable or not required

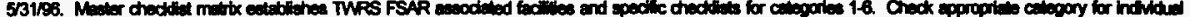

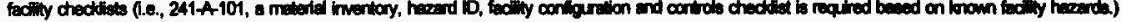

$$
\text { B-121 }
$$


AW FARM

MASTER CHECKST
WHC-SD-WM-HIE-007

Aov. 0

\begin{tabular}{|c|c|c|c|c|c|c|c|c|}
\hline Facility & Typo & $\begin{array}{l}\text { Malverted hwertory, } \\
\text { (Tat 1) }\end{array}$ & Harde $1 D_{2}$ & $\begin{array}{l}\text { Urique Phenoments } \\
\text { (Table } 3 \text { ) }\end{array}$ & $\begin{array}{l}\text { Fecilly Conlugurion } \\
\text { (Table } 4 \text { ) }\end{array}$ & Pad Lovel & 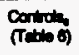 & Comment \\
\hline \multicolumn{9}{|c|}{ 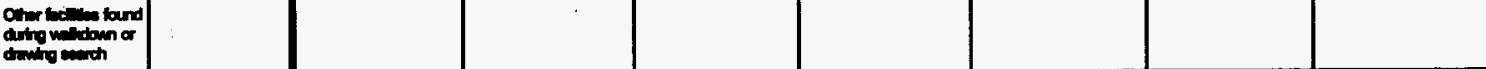 } \\
\hline & Chenge trivior & $x$ & $M$ & $m$ & $x$ & $x$ & $x$ & $M$ \\
\hline & $\begin{array}{l}\text { Acid souver } \\
\text { menhole }\end{array}$ & $x$ & $M$ & M & $x$ & $x$ & $\mathrm{M}$ & 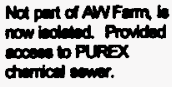 \\
\hline
\end{tabular}


TAELE 1

\begin{tabular}{|c|c|c|c|c|c|c|c|c|}
\hline \multirow{2}{*}{ MAR) C } & \multirow{2}{*}{ Cappocity } & \multicolumn{5}{|c|}{ Muneriel invertory } & \multirow{2}{*}{ antly } & \multirow{2}{*}{ Commerte } \\
\hline & & Crenciliontion & Type & Pryical & Votum or Achity & Trunciert & & \\
\hline 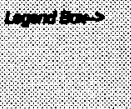 & 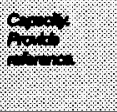 & 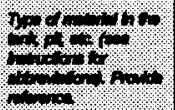 & 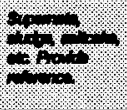 & $5.9 \%$ & $\frac{1}{6}+1 / 4$ & $\frac{6}{4}+\frac{1}{6}$ & 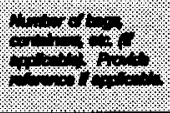 & 18 \\
\hline \multicolumn{9}{|l|}{ 1. Turt conture } \\
\hline 241-AW 101 & $1.18 \mathrm{Mbol} / \mathrm{cs}]$ & DSSF [d1] & spernes [d1] & Mquid jou & $1043 \mathrm{~kg} / \mathrm{d}$ & No & $m$ & $m$ \\
\hline 241AWC101 & $\mathrm{MA}$ & DSsF [d1] & ancld] & and (10) & $4 \mathrm{log} / \mathrm{l}(\mathrm{d}$ & No & $m$ & $\mathrm{~m}$ \\
\hline 241AW-10e & $1.16 \mathrm{Mbol}[\mathrm{ds}]$ & DN [d]] & apenem [dt] & 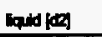 & $30 \mathrm{~kg} / \mathrm{lat}$ & wo & $M$ & MA \\
\hline 241 AW 100 & $M$ & DN \{d1\} & $\operatorname{man}[d]$ & sold deas & $50 \mathrm{log} / \mathrm{d} / \mathrm{A}$ & No & $M$ & $m$ \\
\hline 241AN<103 & $1.18 \mathrm{Mbed}[\mathrm{dP}]$ & DNPD [d1] & apemelo [d1] & Wid & $149 \mathrm{kod} / \mathrm{l}$ & No & $m$ & $m$ \\
\hline 241AWN103 & MA & DNFD [di] & [d1] & $\operatorname{sold} 1004$ & $303 \mathrm{kged} 1004$ & is & $m$ & $\mathrm{~m}$ \\
\hline 241AW 104 & $1.16 \mathrm{Mbot} / \mathrm{KO}$ & DN [d1] & apernem [d1] & Nopid (x) & $034 \mathrm{kged}$ & No & $m$ & $M$ \\
\hline 241AW:104 & Ma & DN [d1] & [d1] & $\cos (\log$ & $200 \mathrm{knd}$ & No & $m$ & $\mathrm{ma}$ \\
\hline 241-AWK105 & 1.16 Moel lats] & Durp [d] & apanda [di] & 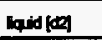 & $130 \mathrm{kow} / \mathrm{k}$ & No & $m$ & $m$ \\
\hline 241-AN/105 & $\mathrm{MA}$ & DAPO [d1] & [d1] & coldid & $200 \mathrm{kgol} / \mathrm{kat}$ & no & m & $m$ \\
\hline 241 AW 108 & $1.16 \mathrm{Mod} / \mathrm{ldag}$ & DN [d1] & apennulo [d1] & 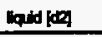 & 3001000 & No & $m$ & $m$ \\
\hline 211AN-106 & $m$ & ON [di) & anceles [dI] & $\operatorname{cotad} \mid d x$ & 2200 kod lot & No & $m$ & $m$ \\
\hline & & & & & & & & \\
\hline
\end{tabular}


TAEUE 1

\begin{tabular}{|c|c|c|c|c|c|c|c|c|}
\hline \multirow{2}{*}{$\begin{array}{l}\text { Monorid at Fiak } \\
\text { (MAR) Cancory }\end{array}$} & \multirow{2}{*}{ Cepecity } & \multicolumn{5}{|c|}{ Mneried Imentery } & \multirow{2}{*}{ arrity } & \multirow{2}{*}{ Commert } \\
\hline & & Cremitiontion & Type & $\begin{array}{l}\text { Phyliced } \\
\text { Form }\end{array}$ & Votume or Adivily & Trunient & & \\
\hline $60.10 .0 \%$ & opins & 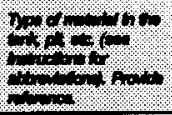 & 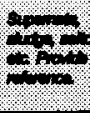 & $\frac{1}{4-1}+19=$ & $\frac{1}{4}+1+1$ & $\frac{6}{6}$ & 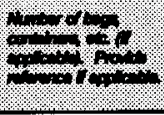 & 10 \\
\hline \multicolumn{9}{|c|}{2 Tork Heachenes } \\
\hline 241 AM 101 & $\mathbf{M A}$ & $\mathbf{M}$ & $m$ & Vepor & 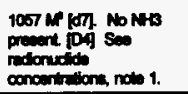 & $\mathbf{m o}$ & MA & $\mathrm{M}$ \\
\hline 241AW-102 & NA & M & $M$ & Veror & 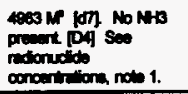 & No & $M$ & $M$ \\
\hline 241A4N100 & $M$ & $M$ & MA & Vepor & 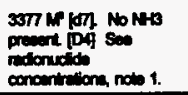 & No & M & $M$ \\
\hline 241AW-104 & $M$ & $\mathbf{M A}$ & $M$ & Vipor & 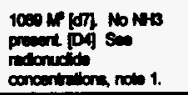 & no & $M$ & $M$ \\
\hline $241-4 \times 105$ & $M$ & MA & $M$ & Veror & 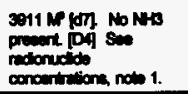 & No & $M$ & $m$ \\
\hline $241-4 W+106$ & $M$ & MA & $M$ & Vuper & 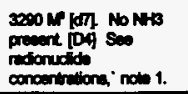 & No & M & $\mathrm{M}$ \\
\hline & & & & & & & & \\
\hline
\end{tabular}

$N A=$ Not applicable or not required $5 / 31 / 96$ 


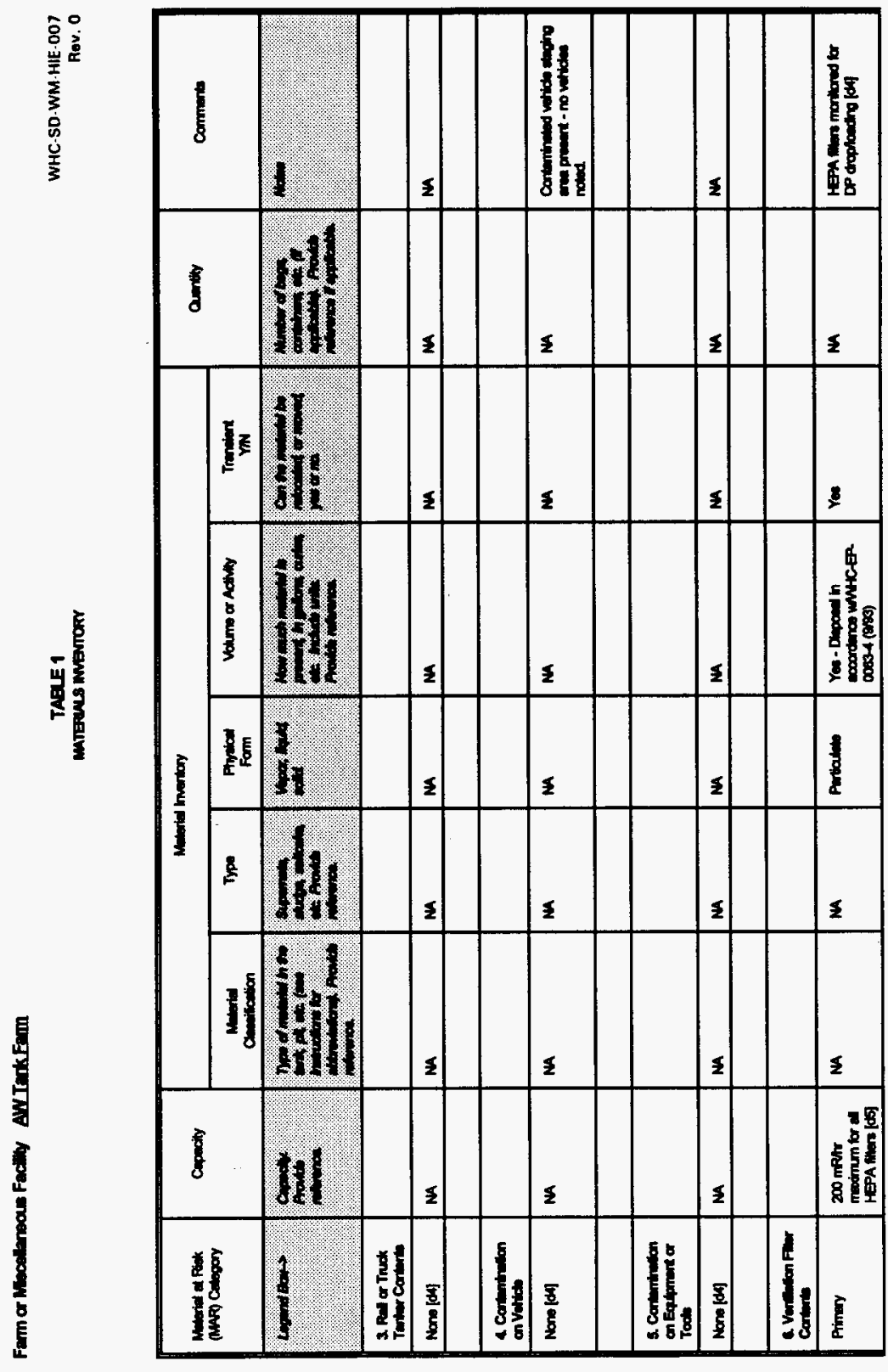

$\frac{1}{6}$ 


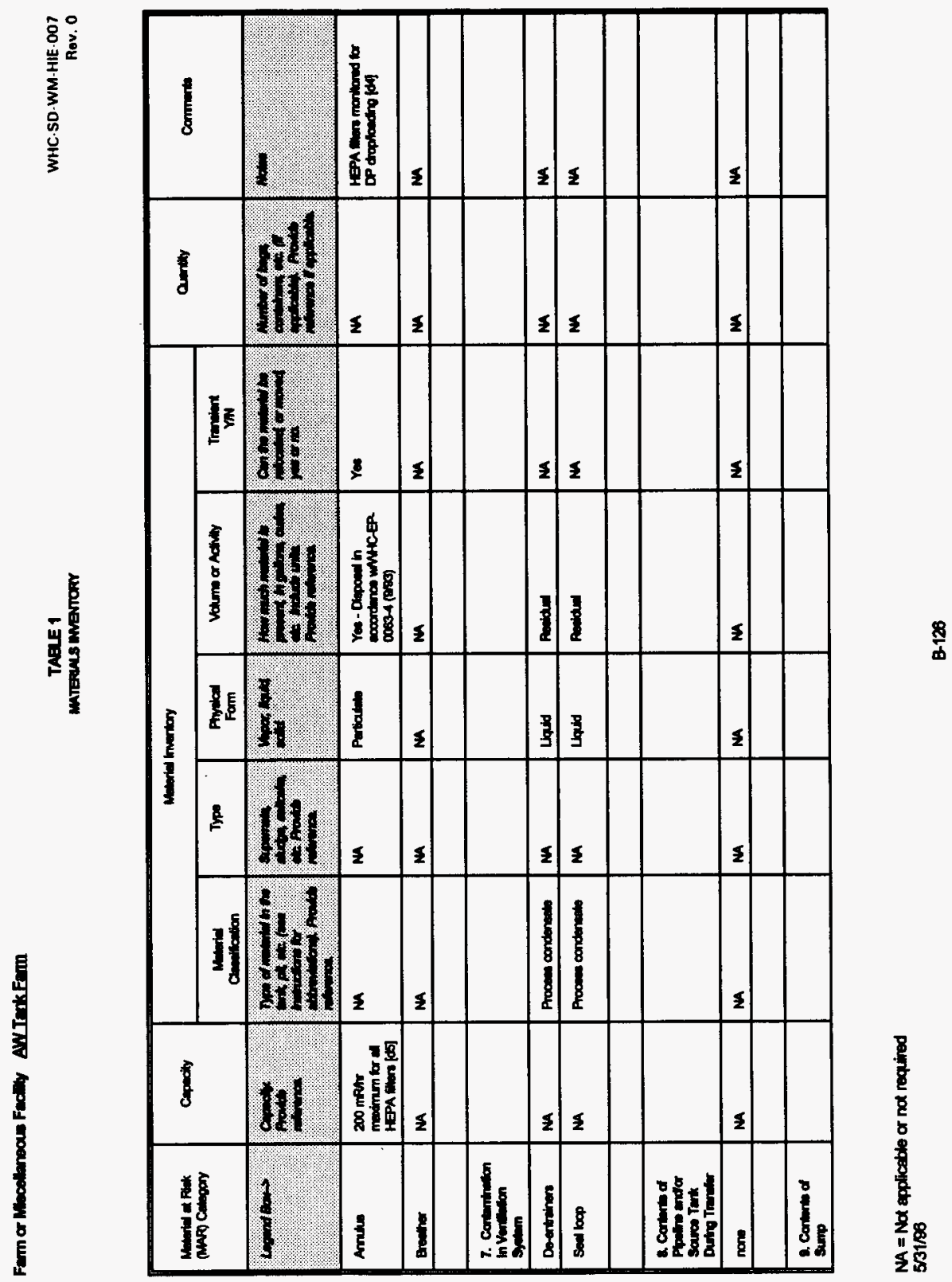




\begin{tabular}{|c|c|c|c|c|c|c|c|c|}
\hline$w$ & and & on & umospn & enperey & $\mathbf{w}$ & 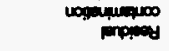 & lozomel & $300 \mathrm{ll}$ dend pees \\
\hline$w$ & 1 & क & mosin & onposer & $\mathbf{w}$ & 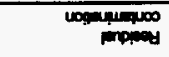 & umasen & 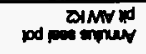 \\
\hline $\mathbf{w}$ & ad b & on & umounn & enpond & $w$ & conimpingos & boned $10 \times 190^{\circ}$ & 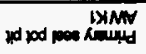 \\
\hline$w$ & ב & w & unoupn & anpond & $\mathbf{W}$ & vompingusos & [Eomed mox or & YMV apd apen \\
\hline$w$ & 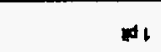 & क & umasen & mpmat & $\mathbf{w}$ & compiniminos & Leones $10 \times 1 \cdot 2$ & 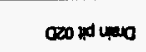 \\
\hline $\mathbf{w}$ & o d & ov & mown & enpond & $\mathbf{w}$ & 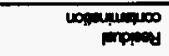 & 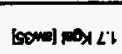 & Do0-910 and dind \\
\hline w & ands & कN & mosen & yiper & $w$ & 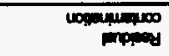 & 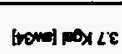 & and dund pavioso \\
\hline $\mathbf{w}$ & ando & 9 & mosenn & enpmes & $w$ & comouningos & 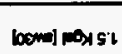 & pod dund somperto \\
\hline & & & & & & & & mox \\
\hline 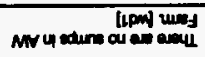 & w & $\mathbf{w}$ & $w$ & w & W & $\mathbf{w}$ & $\mathbf{w}$ & $\mathbf{W}$ \\
\hline :. & (1) & 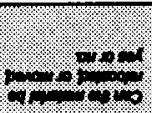 & $\left(\frac{1}{1}\right)$ & 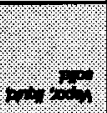 & $\left(\frac{1}{4}\right)$ & 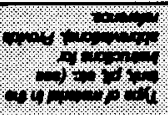 & 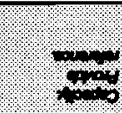 & 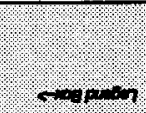 \\
\hline \multirow{2}{*}{ (1) } & \multirow[b]{2}{*}{ ONO } & Nom & Ampor so munan & wo91 & $\infty x_{1}$ & 年 & \multirow{2}{*}{ Apactos } & \multirow{2}{*}{ 1 } \\
\hline & & \multicolumn{5}{|c|}{ 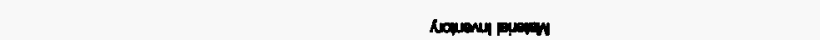 } & & \\
\hline
\end{tabular}

0 ำ

LOO-JHH-WM-OS-JHM

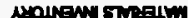

I JIEN/

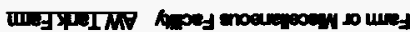




\begin{tabular}{|c|c|c|c|c|c|c|c|c|}
\hline \multirow{2}{*}{ 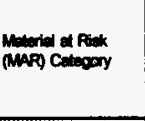 } & \multirow{2}{*}{ Cepecity } & \multicolumn{5}{|c|}{ Meneried inveritory } & \multirow{2}{*}{ andity } & \multirow{2}{*}{ Conmants } \\
\hline & & Cempiticition & Typo & $\begin{array}{l}\text { Phyicul } \\
\text { Form }\end{array}$ & Vothem or Adithy & $\begin{array}{c}\text { Trencient } \\
\text { Yin }\end{array}$ & & \\
\hline 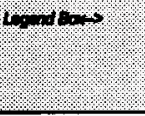 & $\infty_{10}^{\infty}$ & 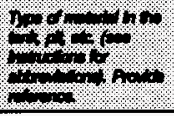 & $\frac{1}{4}$ & $4 \%$ & $\frac{1}{4+1}+1$ & 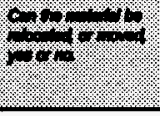 & $\frac{1}{4}$ & : \\
\hline $\begin{array}{l}\text { Vertivion } \\
\text { intrument pits } \\
\text { VPR-1,23 }\end{array}$ & $\begin{array}{l}5.2 \mathrm{Kod} \\
(\mathbf{m} 37,38)\end{array}$ & $\begin{array}{l}\text { Ruidus } \\
\text { conterintion }\end{array}$ & $m$ & Recides & Untoromn & No & 30 & m \\
\hline Fuen ph & $.8 \mathrm{Kog}$ [ [m & 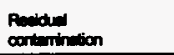 & $\mathrm{m}$ & Renides & Untrown & No & 1 put & $m$ \\
\hline $\begin{array}{l}\text { Chem out bosese } \\
\text { AMr, } 10\end{array}$ & $047 \mathrm{Kod} \mathrm{[mo1]}$ & 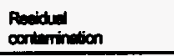 & MA & Renidve & Unteroun & No & 2000 & M \\
\hline & & & & & & & & \\
\hline \multicolumn{9}{|l|}{ 11. $\operatorname{sern} 313$} \\
\hline Oander $[1001$ & 400 ant 100 | & 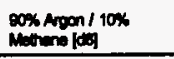 & $m$ & Com ntore & 400 an 600 & Ye & 2 boilles & For PCAME. 10, \\
\hline \multicolumn{9}{|l|}{12000} \\
\hline Senvios buidne & MA & Raun next & m & Reacise & $m$ & no & m & m \\
\hline Change triver & m & Low bovet wededs & SNP: ather low & Sold & Low lover & Yeo & $M$ & 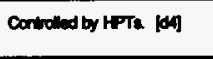 \\
\hline 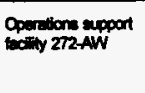 & Seo cormente & See cormment & Sea contment & Sees commente & Ses cormment & Seo cormment & Ses commert & 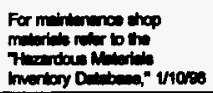 \\
\hline
\end{tabular}




\begin{tabular}{|c|c|c|c|c|c|c|c|c|}
\hline \multirow{2}{*}{ 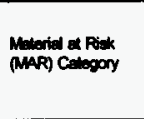 } & \multirow{2}{*}{ Cespecity } & \multicolumn{5}{|c|}{ Materiel inveribry } & \multirow{2}{*}{ amentity } & \multirow{2}{*}{ Comments } \\
\hline & & $\begin{array}{c}\text { Matrial } \\
\text { Clessification }\end{array}$ & Type & $\begin{array}{l}\text { Pryiod } \\
\text { Form }\end{array}$ & Vothme or Activity & $\begin{array}{c}\text { Tremient } \\
\text { Yw }\end{array}$ & & \\
\hline $6,0,0,0,3$ & $\cos _{100}$ & 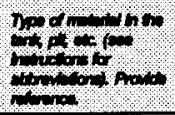 & 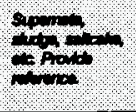 & $v_{0} x^{4}$ & 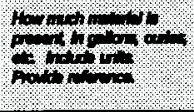 & 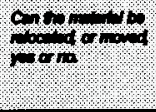 & 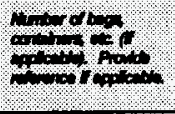 & $\%$ \\
\hline $\begin{array}{l}\text { Add nowor } \\
\text { menthole }\end{array}$ & See cormment & See cormment & see cormment & See cormment & See comment & Soe commont & See comment & 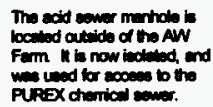 \\
\hline $\begin{array}{l}\text { Sectilito } \\
\text { acoumitation area }\end{array}$ & 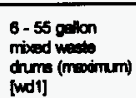 & RCRA mioed westo & 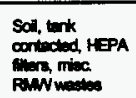 & Solld & $\begin{array}{l}\text { Low Lovel (ness then } \\
\text { denes C) }\end{array}$ & $\overline{Y e s}$ & $\begin{array}{l}8 \text { - } 55 \text { gal dume max } \\
\text { capedily }\end{array}$ & MA \\
\hline & & & & & & & & \\
\hline
\end{tabular}

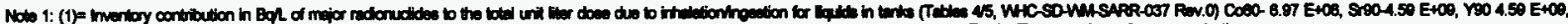

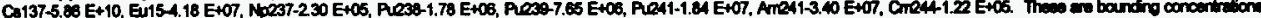

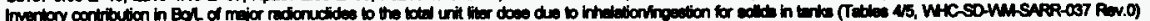

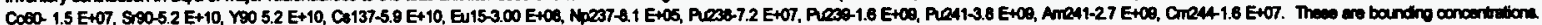

\section{$d 1=$ WHCEP-0182-03}

$Q=$ Tudor $1906,7430-0000$

do = SD-WMSAR-016, Rov 1

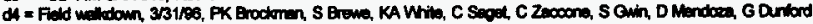

LS = SOMULAR-16

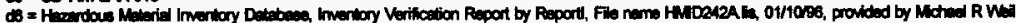

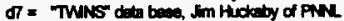

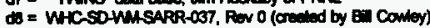

ano $=H-2-905142$ Pev. 1

$a \mathrm{al}=1+1400050211$ Rev. 2

an: $=4+2-7034811$ Rov. 11.

and $=$ H-2-703481 Rov. 15.

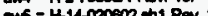

NA = Not applicable or not required

$5 / 31 / 96$ 
TAFUE1

MATERALS INMENTORY
WHC-SD-WM-HIE-007

Rev. 0

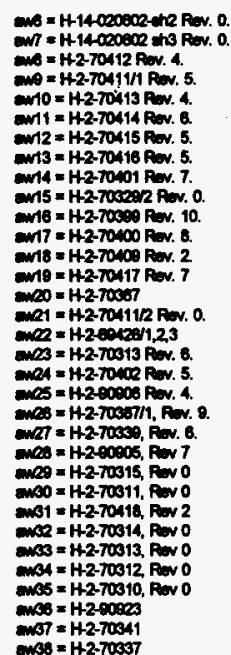

$1437=1+2-7003$

$N A=$ Not applicable or not required

$5 / 3498$ 
TAEIE 2

\begin{tabular}{|c|c|c|c|c|c|c|c|c|c|c|c|c|c|c|c|}
\hline Facity & Typo & $\stackrel{\text { corroens }}{A}$ & $\begin{array}{c}\text { E } \\
\text { Explowine } \\
\text { Prophoric }\end{array}$ & $\begin{array}{c}c \\
\text { Nuclear } \\
\text { Crlicatiny }\end{array}$ & $\frac{\text { D. }}{\text { Finmide }}$ & $\underset{\text { Padiulion }}{E-}$ & $\begin{array}{c}\text { F- } \\
\text { Chem } \\
\text { Poth }\end{array}$ & F & Haction & $\underset{\text { Knntion }}{\text { Kon }}$ & Linelo & $\underset{\substack{\text { Gromily, } \\
\text { Height }}}{K}$ & L- & $\underset{\text { maxpl }}{M}$ & commente \\
\hline \multicolumn{2}{|c|}{$t=10=1$} & \multicolumn{13}{|c|}{ 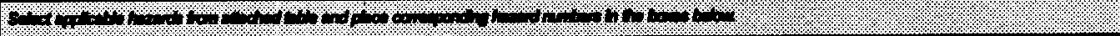 } & $\%$ \\
\hline \multicolumn{2}{|c|}{ MNTAKFAFA } & $m$ & $M$ & MA & $M$ & MA & $m$ & $5(c)$ & $6(0)$ & $2,3(0)$ & $M$ & 11 (c) & $M$ & $\mathbf{M}$ & $M$ \\
\hline 241-An-10 & DST & $2(a)$ & $6,7,8(0)$ & $15(a)$ & $4,11,14$ & 5 (a) & $M(0)$ & $5(c)$ & $\begin{array}{l}\text { 4,6,8,10, } \\
14(c)\end{array}$ & 3 & MA $(G)$ & $11(9)$ & $M(\infty)$ & NA (c) & 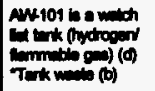 \\
\hline $241-4 \times 100$ & DST & 2 (a) & $6,7,8(a)$ & 15 (a) & $4_{(0)}^{11,14}$ & $5^{2}(a)$ & $M(a)$ & $5(c)$ & $\begin{array}{l}4,6,9,10 \\
14(c)\end{array}$ & 3 & MA(c) & 11 (c) & $M(c)$ & $\mathrm{MA}(\mathrm{C})$ & Tork (b) \\
\hline $24 t+14-100$ & DST & $2(0)$ & $6,7,8(0)$ & 15 (a) & $4,11,14$ & $5^{*}(a)$ & $M(a)$ & $5(c)$ & $\begin{array}{l}4,6,9,10 \\
14(c)\end{array}$ & 3 (c) & $M$ (c) & 11 (c) & MA $(c)$ & $\mathrm{M}(\mathrm{c})$ & Tonk wom (이 \\
\hline 241-4M-104 & DST & $2(0)$ & 6, 7, 8 (a) & $15(0)$ & $\begin{array}{l}4,11,14 \\
(0)\end{array}$ & $5^{*}(0)$ & NA(a) & $5(c)$ & $\begin{array}{l}4,6,9,10 \\
14(c)\end{array}$ & 3 (c) & $M(c)$ & $11(c)$ & MA (c) & $M(c)$ & 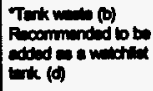 \\
\hline $241-4 \times 105$ & DST & $2(0)$ & $6,7,8(a)$ & 15 (a) & $4,11,14$ & $5^{\circ}($ ) & MA (a) & $5(c)$ & $\begin{array}{l}4,6,9,10, \\
14(c)\end{array}$ & 3 (c) & $M(c)$ & $11(c)$ & $M(c)$ & MA(A) & Trak wam (b) \\
\hline 241 AMt 105 & DST & $2(\theta)$ & $6,7,8(a)$ & $15(0)$ & $\begin{array}{l}4,11,14 \\
(4)\end{array}$ & $5^{*}(1)$ & $\mathrm{MA}(0)$ & 5 (c) & $\begin{array}{l}4,6,9,10 \\
14(c)\end{array}$ & $3(C)$ & $M(c)$ & 11 (C) & $M(c)$ & $M A(c)$ & Tark was (b) \\
\hline cosminos & Creanout bou & 2 (a) & $6,7,8(a)$ & NA & $M$ & $M$ & $M$ & $m$ & $4(c)$ & $\mathrm{M}$ & M & $M$ & $M$ & $M$ & M \\
\hline Cosmasio & Creenout box & $2(a)$ & $6,7,8(a)$ & NA & $M$ & $M$ & $M$ & $\mathrm{M}$ & $4(9)$ & $M$ & M & $\mathrm{MA}$ & $m$ & $m$ & $M$ \\
\hline 241-AKro1 & $\begin{array}{l}\text { Comproser } \\
\text { bulling }\end{array}$ & $\mathbf{M}(c)$ & $M(c)$ & NA(c) & $4,5,10(c)$ & $\mathrm{MA}(\mathrm{C})$ & M & $\begin{array}{l}1,2,3,5 \\
(C)\end{array}$ & $\begin{array}{l}4,8,9, \\
10,11, \\
14,10 \text { (c) }\end{array}$ & $2,4(9)$ & $M(c)$ & $M(c)$ & $0,8(c)$ & $3.6(c)$ & 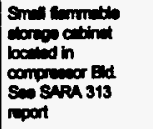 \\
\hline
\end{tabular}

NA = Not applicable or not required $5 / 31 / 86$ 


\begin{tabular}{|c|c|c|c|c|c|c|c|c|c|c|c|c|c|c|c|}
\hline Faciliny & Type & Comoine & \begin{tabular}{|c|} 
B- \\
Exploetved \\
Pyrophoric
\end{tabular} & $\begin{array}{l}\text { C. } \\
\text { Nucteor } \\
\text { Criticatity }\end{array}$ & 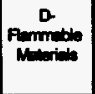 & $\underset{\text { Tharidic }}{E-}$ & $\underset{\text { Rom }}{\mathrm{F}-}$ & $\begin{array}{c}G \\
\text { Friction }\end{array}$ & $\stackrel{H}{H}$ & $\begin{array}{c}\text { Knetio } \\
\text { Rotition }\end{array}$ & $\underset{\text { Linem }}{\tan }$ & $\operatorname{ming}_{\text {Grity. }}^{K}$ & 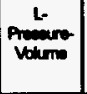 & $\underset{\substack{\text { Thame } \\
\text { nadient }}}{M}$ & Commenta \\
\hline \multicolumn{2}{|c|}{ 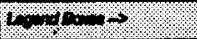 } & \multicolumn{13}{|c|}{ 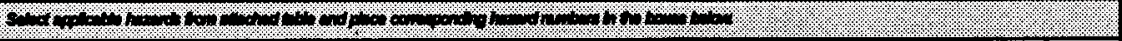 } & $\%$ \\
\hline $211-A N 025$ & $\begin{array}{l}\text { Foed pimp } \\
\text { pit }\end{array}$ & $2(b)$ & $M$ (b) & 15 (b) & NA (b) & MA (b) & NA(b) & $5(\mathrm{c})$ & $0,14(C)$ & $3(\mathrm{c})$ & $M$ (c) & $M(C)$ & MA (c) & $M(G)$ & 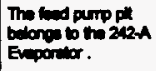 \\
\hline $241 \mathrm{AW}$ & Fum pot & MA (o) & M(b) & MA (b) & MA(b) & $\mathrm{M}(\mathrm{b})$ & MA(b) & $M(b)$ & 14 (b) & M(b) & $M(b)$ & M(b) & MA(b) & M(b) & Lewh dition in \\
\hline 241-AK271 & $\begin{array}{l}\text { Intrument } \\
\text { buting }\end{array}$ & MA (c) & MA (c) & MA (c) & $N A(c)$ & $M A(C)$ & $\mathrm{MA}(\mathrm{c})$ & $M(c)$ & $\begin{array}{l}4,3,16 \\
5,6,7 \\
6,11,14 \\
\text { (c) }\end{array}$ & MA (c) & $M A(c)$ & NA(c) & $5(c)$ & 3 (c) & $\mathrm{M}$ \\
\hline 241-AW272 & $\begin{array}{l}\text { Opontione } \\
\text { apport } \\
\text { tocility }\end{array}$ & $\begin{array}{l}\text { See } \\
\text { corment }\end{array}$ & $M$ & $\mathrm{MA}$ & M & $m$ & MA & $\mathrm{MA}$ & MA & $M$ & $M$ & MA & $m$ & $M$ & 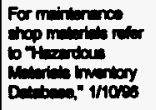 \\
\hline 2a1-AnK273 & $\begin{array}{l}\text { Senvice } \\
\text { building }\end{array}$ & $M(c)$ & $M(c)$ & MA (c) & $M(c)$ & $M A_{(c)}$ & $M A$ (c) & $M(c)$ & $11,14(c)$ & M(c) & $M A(c)$ & $M(c)$ & $M(c)$ & $3(c)$ & spoce hivir in \\
\hline 241-ANA & Vhe pit & 2 (b) & MA (b) & $15(b)$ & MA (b) & MA (b) & $M(b)$ & 5 (b) & 14 (b) & M(b) & $m(b)$ & $M(0)$ & MA (b) & Na (b) & $m$ \\
\hline \multirow[t]{2}{*}{ 241-AKB } & Whe pl & 2 (b) & $M(b)$ & $15(b)$ & $M(b)$ & MA (b) & $M(b)$ & 5 (b) & $14(0)$ & $M(b)$ & $\mathrm{MAO}$ & $M(b)$ & M (b) & MA(b) & $\mathrm{M}$ \\
\hline & $\begin{array}{l}\text { Sencinis } \\
\text { Acounia } \\
\text { ton Area }\end{array}$ & 1,24 & MA & MA & $\begin{array}{l}2.10 . \\
170 \text {. } \\
\text { contind }\end{array}$ & $M$ & $m$ & $M$ & $m$ & $M$ & $m$ & $M$ & M & $M$ & $M$ \\
\hline & & & & & & & & & & & & & & & \\
\hline
\end{tabular}

(a) $=$ CSDT-151-00007

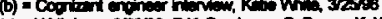

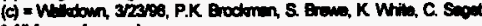

All fran riverenco b

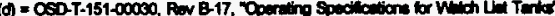

NA $=$ Not applicable or not required $5 / 31 / 98$ 


\begin{tabular}{|c|c|c|c|c|c|c|c|c|c|c|c|c|c|}
\hline \multirow[t]{2}{*}{ Faciliny } & \multirow[t]{2}{*}{ Type } & \multirow{2}{*}{$\underset{\mathbf{C}=}{\text { Flomplo }}$} & \multirow[t]{2}{*}{ Fencoynide } & \multicolumn{3}{|c|}{ Oganics } & \multirow{2}{*}{$\begin{array}{c}\text { Hon Hod } \\
(070,000 \\
\text { BTum) }\end{array}$} & \multirow[t]{2}{*}{ Pyophatios } & \multirow[t]{2}{*}{ Siphoning } & \multirow{2}{*}{$\begin{array}{l}\text { Criceny } \\
\text { Pled in } \\
\text { scils }\end{array}$} & \multirow{2}{*}{ 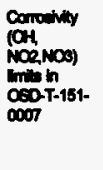 } & \multirow[t]{2}{*}{ Other } & \multirow[t]{2}{*}{ Commots } \\
\hline & & & & Sepertion & $\begin{array}{l}\text { Toch } \\
\text { liquth } \\
\text { (P10 ol) }\end{array}$ & $\begin{array}{l}\text { roc in } \\
\text { ectids } 3 \\
\text { whis (dy } \\
\text { beis) }\end{array}$ & & & & & & & \\
\hline \multicolumn{2}{|c|}{$t \cdot 1)^{2}$} & ${ }_{\infty}^{\infty}$ & 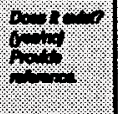 & $\log _{\sin }$ & 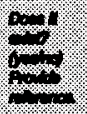 & . & $\frac{1}{4}$ & 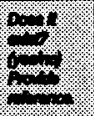 & (1) & . & \% & $\frac{1}{4 \%}$ & mol \\
\hline \multicolumn{14}{|c|}{$6+15+4$} \\
\hline${ }_{101}^{211-A M}$ & DST & 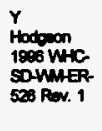 & $\begin{array}{l}\text { N } \\
\text { Bernain } 8 \\
\text { Simpeon } 1991 \\
\text { WHCSD } \\
\text { WWER-133 } \\
\text { Rov. } 0\end{array}$ & 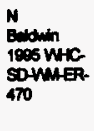 & 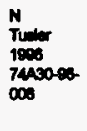 & 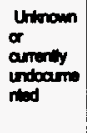 & 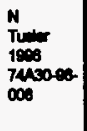 & $\begin{array}{l}N \\
\text { pemond } \\
\text { communicat } \\
\text { lon win } \\
\text { Den } \\
\text { Renoids }\end{array}$ & 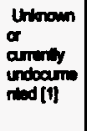 & 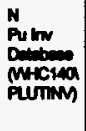 & 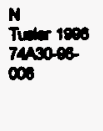 & $M$ & 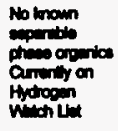 \\
\hline $241-4 M$ & DST & 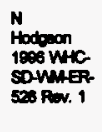 & $\begin{array}{l}\text { N } \\
\text { Eontein \& } \\
\text { Sinpeon } 1891 \\
\text { WHCSO } \\
\text { WWER-133 } \\
\text { Rov. O }\end{array}$ & $\begin{array}{l}\text { N } \\
\text { Exch } 1805 \\
\text { WHCSD } \\
\text { WMOP-149 }\end{array}$ & $\begin{array}{l}\text { N } \\
\text { Tumeser } \\
1906 \\
7440-86- \\
000\end{array}$ & $\begin{array}{l}\text { Untonoun } \\
\text { of } \\
\text { andery } \\
\text { undeam } \\
\text { nited }\end{array}$ & 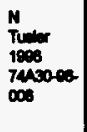 & 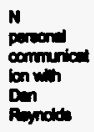 & 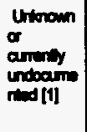 & $\begin{array}{l}\text { N } \\
\text { Pu hw } \\
\text { Dubas } \\
\text { Muc14a } \\
\text { Pumis }\end{array}$ & $\begin{array}{l}n \\
\text { Tuler 1eces } \\
7400-68 \\
\infty \infty\end{array}$ & $M$ & $\begin{array}{l}\text { No known } \\
\text { theme oremices }\end{array}$ \\
\hline 2019 $_{100}$ & DST & $\begin{array}{l}\text { N } \\
\text { Hodpon } \\
1000 \text { WHC } \\
\text { SOWHER- } \\
500 \text { Row. } 1\end{array}$ & 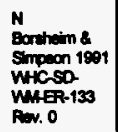 & $\begin{array}{l}\text { N } \\
\text { Hodtown } \\
1906 \text { Whic } \\
\text { SOWWER- } \\
450\end{array}$ & $\begin{array}{l}\text { N } \\
\text { Tumer } \\
1900 \\
7408000 \\
000\end{array}$ & 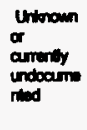 & 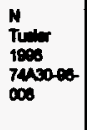 & 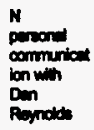 & $\begin{array}{l}\text { Untroun } \\
\text { or } \\
\text { anvely } \\
\text { uncoaim } \\
\text { rivd [t] }\end{array}$ & 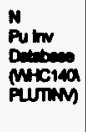 & 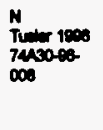 & $M$ & No known \\
\hline
\end{tabular}

$N A=$ Not applicable or not required 
TABLE 3

UNAL PHEVOM JUA CHECKUST

\begin{tabular}{|c|c|c|c|c|c|c|c|c|c|c|c|c|c|}
\hline \multirow[t]{2}{*}{ Facility } & \multirow[t]{2}{*}{ Type } & \multirow{2}{*}{ Flanmebolo } & \multirow[t]{2}{*}{ Ferrogyanide } & \multicolumn{3}{|c|}{ Orgenica } & \multirow{2}{*}{ 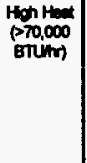 } & \multirow[t]{2}{*}{ Prophorica } & \multirow[t]{2}{*}{ Sphoning } & \multirow{2}{*}{$\begin{array}{l}\text { Citicany } \\
\text { (Pl ph in } \\
\text { olide) }\end{array}$} & \multirow{2}{*}{ 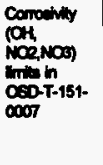 } & \multirow[t]{2}{*}{ Other } & \multirow[t]{2}{*}{ Comments } \\
\hline & & & & Soperibes & $\begin{array}{l}\text { roc in } \\
(10 \mathrm{~d} \text { ) }\end{array}$ & 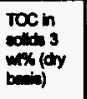 & & & & & & & \\
\hline $10 \% 14$ & & 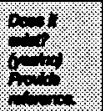 & (1) & $\frac{1}{6}$ & $\frac{1}{1+1}+1$ & $\frac{1}{6}$ & (2) & 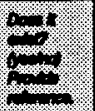 & 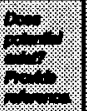 & \% & \%or & 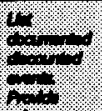 & \\
\hline $\begin{array}{l}241-4 m \\
104\end{array}$ & DST & $\begin{array}{l}Y \\
\text { Hoctoon. } \\
1906 \text { WHC } \\
\text { SDWMER- } \\
\text { S28 Rov. } 1\end{array}$ & $\begin{array}{l}\text { N } \\
\text { Bornhein \& } \\
\text { Simpoon } 1991 \\
\text { WHCSO } \\
\text { WHER-133 } \\
\text { Rov. } 0\end{array}$ & 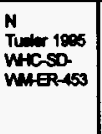 & $\begin{array}{l}\text { N } \\
\text { Tumer } \\
1006 \\
7400-86- \\
\infty 08\end{array}$ & $\begin{array}{l}\text { Untromn } \\
\text { a } \\
\text { currenty } \\
\text { undocame } \\
\text { ntud }\end{array}$ & 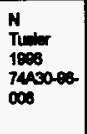 & 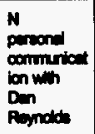 & 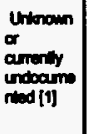 & $\begin{array}{l}\text { N } \\
\text { Pu inw } \\
\text { Duben } \\
\text { Mutcisa } \\
\text { Puming }\end{array}$ & $\begin{array}{l}N \\
\text { Twerr } 1808 \\
740008 \\
\infty 00\end{array}$ & $m$ & 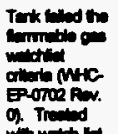 \\
\hline $\begin{array}{l}241-n x^{2} \\
105\end{array}$ & DST & 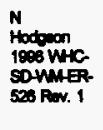 & $\begin{array}{l}\text { N } \\
\text { Exthein a } \\
\text { Simpeon } 1891 \\
\text { WHCSD. } \\
\text { WuER-133 } \\
\text { Rov. } 0\end{array}$ & $\begin{array}{l}N \\
\text { Exch 1905 } \\
\text { WHC-sD } \\
\text { nuOP-146 }\end{array}$ & Deten not & $\begin{array}{l}\text { N } \\
\text { Tueler } \\
1908 \\
7403000 \\
000\end{array}$ & $\begin{array}{l}\text { N } \\
\text { Tumer } \\
1906 \\
743006- \\
008\end{array}$ & 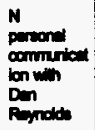 & 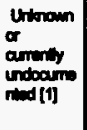 & 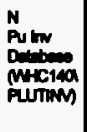 & 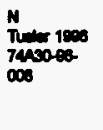 & $M$ & No knom \\
\hline $\begin{array}{l}\text { 241-nin } \\
105\end{array}$ & DST & 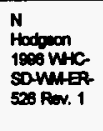 & $\begin{array}{l}\text { N } \\
\text { Boningim } 8 \\
\text { Sinpon } 1801 \\
\text { WhC-SO. } \\
\text { WHER-133 } \\
\text { Rov. } 0\end{array}$ & $\begin{array}{l}N \\
\text { Exh } 1905 \\
\text { WHC SD } \\
\text { WMOP-147 }\end{array}$ & $\begin{array}{l}N \\
T \text { Tumor } \\
1800 \\
7443008 \\
008\end{array}$ & 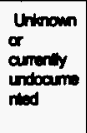 & $\begin{array}{l}\text { N } \\
\text { Tumber } \\
1906 \\
740006 \\
000\end{array}$ & 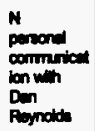 & 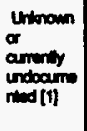 & $\begin{array}{l}N \\
\text { Pum } \\
\text { Dumbar } \\
\text { Muci4a } \\
\text { PUTmin }\end{array}$ & 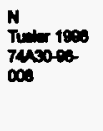 & $M$ & No known \\
\hline
\end{tabular}

$N A=$ Not applicable or not required $531 / 96$ 


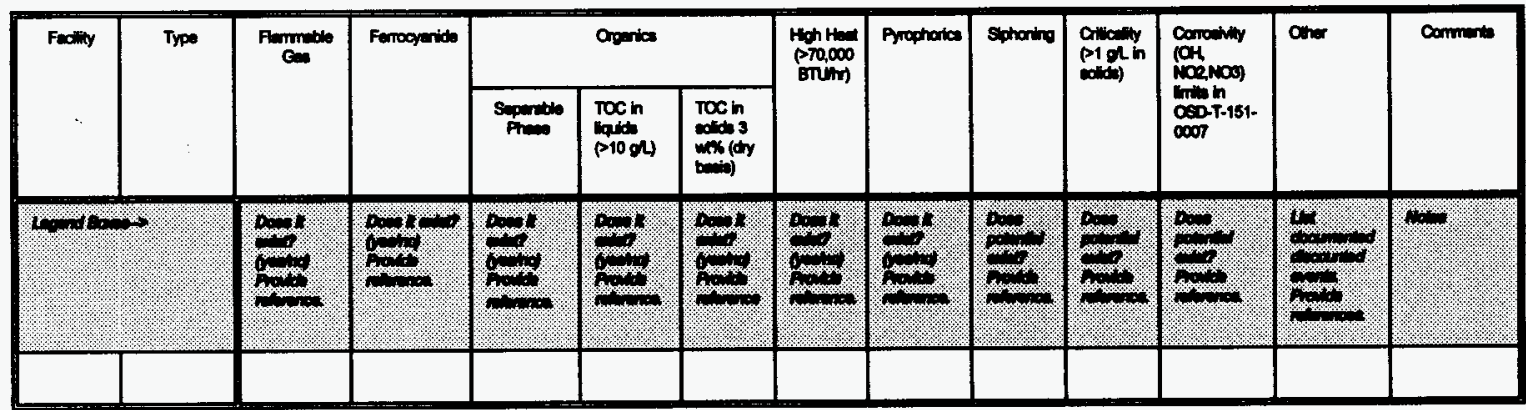

[1] Goup Hezerds Analysis performing independent siphoring evaluation study. 


\begin{tabular}{|c|c|c|c|c|c|}
\hline & & & & & 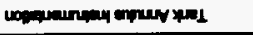 \\
\hline $\mathbf{w}$ & [tp]s & $\operatorname{lom} \mid \infty x$ & $\operatorname{tow} \cos$ & $w$ & ancout \\
\hline$w$ & [LP|s s & $\ln \mid=0$ & tow $\sigma_{\text {A }}$ & LobtMm & 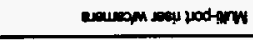 \\
\hline$w$ & LIPIs & {$[\mathrm{pm}]=0$} & 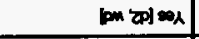 & 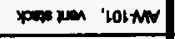 & 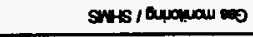 \\
\hline$w$ & LLP'S & (pow) $=0$ & 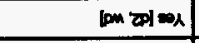 & $w$ & 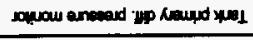 \\
\hline 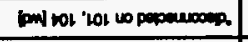 & [LP] & Ind AN & 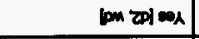 & w & capd pren ley \\
\hline $\mathbf{w}$ & $w$ & $w$ & w & mapringes & (topose) mal pron \\
\hline$w$ & Lopis & $|\mathrm{p} w|=\mathrm{x}$ & $\operatorname{ton} 200100$ & 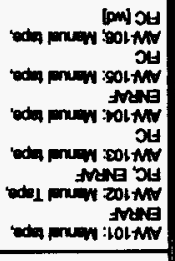 & (Nowed) men pron \\
\hline$w$ & [LP] 9 & pw $=0$ A & Ion $201 \infty \lambda$ & $w$ & 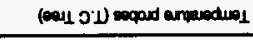 \\
\hline 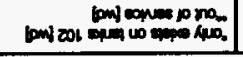 & ILP]s & I PNI & Im & $w$ & 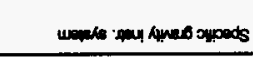 \\
\hline & & & & & 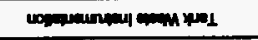 \\
\hline & & & & & 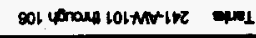 \\
\hline 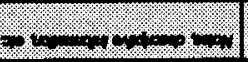 & 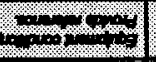 & . & . & 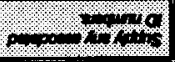 & א. \\
\hline & mingen & apsodo & remodanepers & 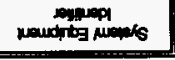 & 仵 \\
\hline
\end{tabular}

$0 \cdot \operatorname{seg}$

100-3IH-WM-OS-כHM

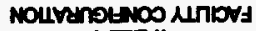

บอก:1 
perrber pu so equaydds PN = $\forall$

\begin{tabular}{|c|c|c|c|c|c|}
\hline & & & & & 20 \\
\hline 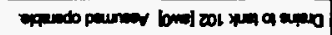 & $w$ & $\infty$ & beom $\operatorname{ma}$ & $\mathbf{w}$ & appo \\
\hline $\mathbf{w}$ & $w$ & low $\operatorname{sol}$ & bognes ind & $\mathbf{w}$ & $\operatorname{lng}$ \\
\hline$W$ & $w$ & $w$ & क & w & pounens \\
\hline 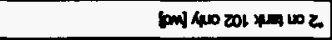 & $w$ & $\mid \operatorname{low} \infty x$ & $\operatorname{lom}, \infty x$ & w & 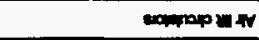 \\
\hline$w$ & IaOIs & $w$ & 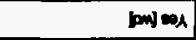 & $w$ & 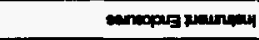 \\
\hline avem 61 & [(t)! & $|p m| \infty \lambda$ & $\operatorname{lom} n=1 \times 1 \times$ & $w$ & 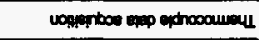 \\
\hline & & & & & ס \\
\hline 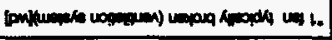 & [LP]s & ponles & $\operatorname{lom} \infty \lambda$ & $w$ & 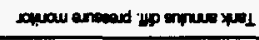 \\
\hline$w$ & (비) $\mathrm{s}$ & $|\ln |=0$ & 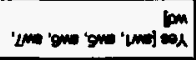 & w & enpeoduen \\
\hline 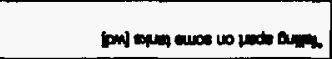 & $w$ & 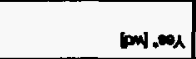 & $\operatorname{low} \cos$ & $\mathbf{w}$ & 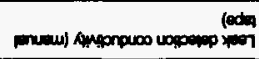 \\
\hline$w$ & [LD] & 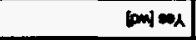 & 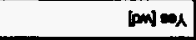 & $w$ & 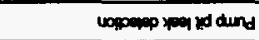 \\
\hline$w$ & (twis) & $\operatorname{son} \mid=0$ & {$[\mathrm{pm} z \mathrm{zp}] \operatorname{mox}$} & $\mathbf{w}$ & 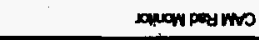 \\
\hline 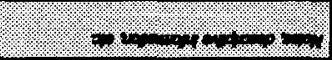 & 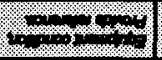 & 10 & 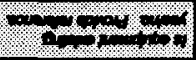 & 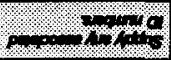 & $\%$ \\
\hline (1) & uopous & 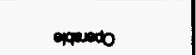 & nemolosemp & wantiog & 酐 \\
\hline
\end{tabular}

100-3H-WM-as-JHM 


\begin{tabular}{|c|c|c|c|c|c|}
\hline $\mathbf{m}$ & $w$ & 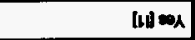 & ItU $\infty$ & w & andead $x$ annus \\
\hline$w$ & $w$ & (I) $=0$ A & [tall ens & w & 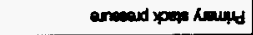 \\
\hline$w$ & |[L]s $s$ & $|\operatorname{low}| \boldsymbol{\infty}$ & $\mid p m$ ' $|p| \mathbf{m a}$ & $w$ & unce mpue of chual yous \\
\hline$w$ & $|t p| s$ & 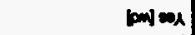 & $|p m i p| \oplus \lambda$ & $w$ & 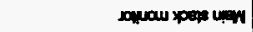 \\
\hline \multicolumn{6}{|r|}{ 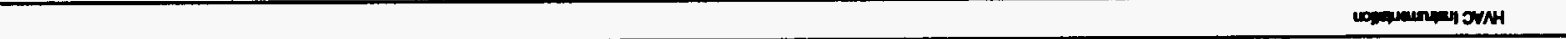 } \\
\hline w) & [LP]s & 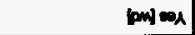 & $\mid p m$ 'Ip] $\infty \lambda$ & $w$ & rant \\
\hline 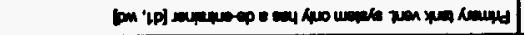 & [LP]s & $|p w| m$ & 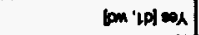 & $\mathbf{w}$ & senpapect \\
\hline 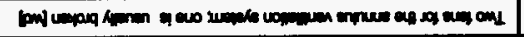 & [LP] $\mathbf{s}$ & Ind on & (pm itpl $=1$ & $\mathbf{w}$ & anst \\
\hline 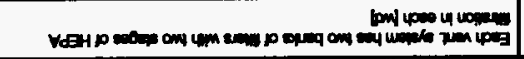 & {$[(\omega) \mid s$} & 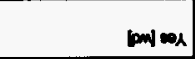 & 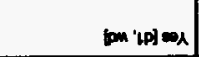 & w & 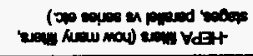 \\
\hline 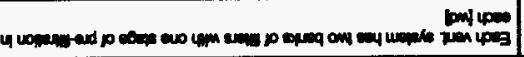 & [Lp]s $\mathbf{s}$ & 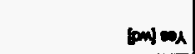 & {$[m|p|=\lambda$} & $w$ & 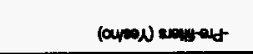 \\
\hline$|p w| x \mid y=1$ & l(f)! & 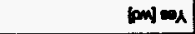 & {$[\mathrm{pw}) \infty \mathrm{N}$} & $w$ & 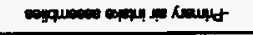 \\
\hline 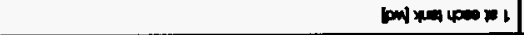 & ILPIS & 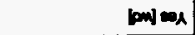 & I pow $\infty x$ & w & 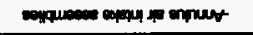 \\
\hline$w$ & ltpls & $|p \mathrm{pw}| \boldsymbol{N}$ & $p w i=x$ & WN & Rompompuen moups \\
\hline 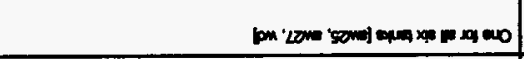 & [IP] 9 & 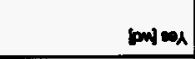 & Ion gove govel ax & $\mathbf{w}$ & 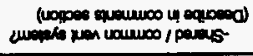 \\
\hline$w$ & lipls $\mathbf{s}$ & $p m=1$ & 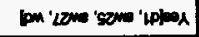 & $\mathbf{W}$ & nepr \\
\hline $\mathbf{w}$ & $\mathbf{w}$ & $\mathbf{m}$ & Ipm 'zone seven 'IPI ow & $\mathbf{W}$ & onpered \\
\hline 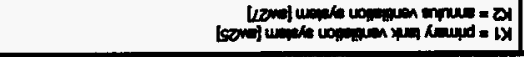 & Lupls & $|p w|=\lambda$ & 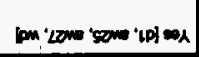 & 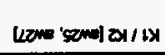 & (o)werels wongunen \\
\hline 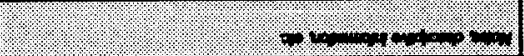 & 1.20 & 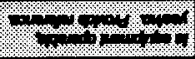 & 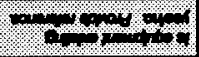 & $5.1 \% 19$ & $p=1$ \\
\hline 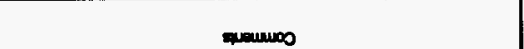 & $\operatorname{mompos}_{\operatorname{mos}}$ & Daped & rosadanplas & 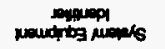 & wants \\
\hline
\end{tabular}




\begin{tabular}{|c|c|c|c|c|c|}
\hline & & & & & and \\
\hline$w$ & ILP]s & $p$ pul $=0$ & $|\operatorname{low}| \cos$ & $w$ & armoad monues \\
\hline$w$ & $\mid(\omega) \mathrm{s}$ & 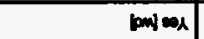 & $|p w|=0$ & w & mo- \\
\hline was an 48 & [LP] $\mathbf{s}$ & 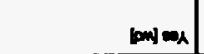 & $|p N|=\alpha$ & $-\infty=Z+เ$ - & A \\
\hline$w$ & an & umasen & umown & $w$ & 40 \\
\hline$w$ & (LPP) & unowen & umoum & $w$ & Meuep pior \\
\hline$w$ & (LP)s & 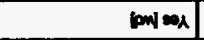 & Ibow exp & atrative & Had pes then \\
\hline 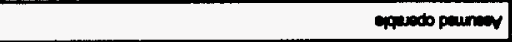 & [Lp]s & $\infty$ & bean $10 x$ & $w$ & cons wor \\
\hline apeodo peanners & $w$ & $\infty$ & 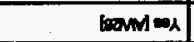 & $w$ & want \\
\hline 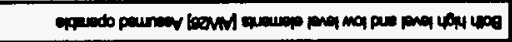 & โLp]s & $\infty$ & $\operatorname{tg} x=10$ & $w$ & nowep por \\
\hline w & $w$ & $w$ & mon ow & $w$ & voposep xwor \\
\hline$w$ & $w$ & $|p m| \infty$ & $\operatorname{lom} \infty \cos$ & IXtMVive & 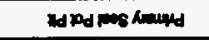 \\
\hline 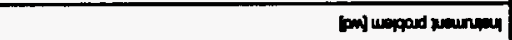 & ILP]s & Ipolas & $|\operatorname{pon}| \infty x$ & $w$ & 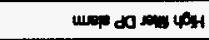 \\
\hline 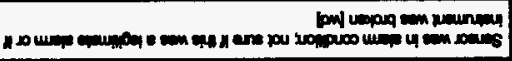 & [HF]s] & pwow & 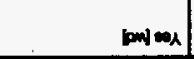 & $w$ & 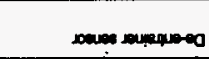 \\
\hline 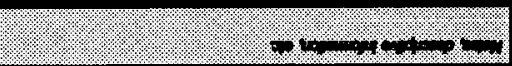 & $4=\infty \%$ & 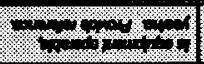 & $2 \%$ & $1.0 .1 \% 9$ & 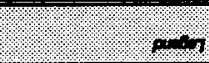 \\
\hline Sos & umpues & 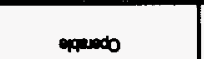 & Inodanaxg & 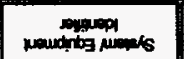 & wevels \\
\hline
\end{tabular}




\begin{tabular}{|c|c|c|c|c|c|}
\hline$w$ & $w$ & $\mathbf{w}$ & $w$ & $w$ & IA $\mathbf{m i}$ \\
\hline$w$ & $w$ & $w$ & $\mathbf{w}$ & $\mathbf{w}$ & 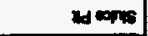 \\
\hline$w$ & w & $m$ & $w$ & $w$ & $x+\cos x \min 0$ \\
\hline 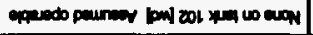 & w & $\infty$ & 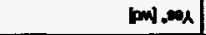 & w & ampunes kenses \\
\hline eqpecto peuner| & $w$ & $\infty$ & ime zwaj ma & $w$ & $\sin$ dunt \\
\hline opquedo pauniner & $w$ & ma & 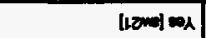 & $w$ & Aade wd expor \\
\hline 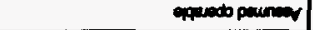 & m & $m$ al & 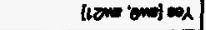 & $\mathbf{w}$ & Lep soo \\
\hline noverem am on 1 & [(t)]s & 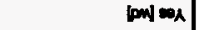 & 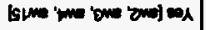 & $\mathbf{w}$ & ropoenep pert \\
\hline w & $\mathbf{w}$ & 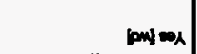 & 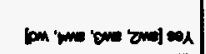 & - conars viom & 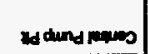 \\
\hline$w$ & $m$ & क⿻ & 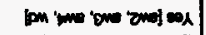 & arow & sow dund \\
\hline 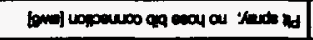 & $w$ & $\operatorname{son} 1$ ex & bomed $\infty$ & $\mathbf{w}$ & 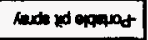 \\
\hline$w$ & (LP] $\mathbf{s}$ & $|\operatorname{low}| \infty \lambda$ & 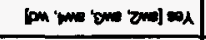 & $w$ & nopoup yor \\
\hline w) & $w$ & $w$ & क⿻ & w & durs- \\
\hline apendo paunen & $w$ & $-\alpha$ & 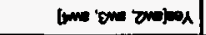 & $\mathbf{W}$ & upp not \\
\hline $\mid p n$ ond & m & {$[\mathrm{PW}]=0 \mathrm{~A}$} & 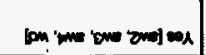 & $\begin{array}{r}980 \\
-4 \operatorname{lom} \text { glom } 152\end{array}$ & W dend mpery \\
\hline 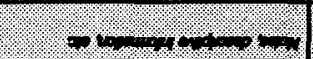 & 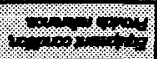 & 40 & 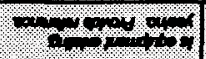 & 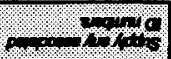 & $P=7$ \\
\hline mancos & monow & apedo & romadarapg & 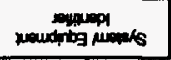 & $2 / 3$ \\
\hline
\end{tabular}

0 'AOY

100-3IH-WM-OS-JHM nouvanotinoo unives

t ITaV 1 
TAEEE 4

FACIUTY CONFIGURATION

WHC-SD-WM-HIE-007 Rov. 0

\begin{tabular}{|c|c|c|c|c|c|}
\hline Spatem & Syam/ Equipment & Exlowoprocent: & Opercte & $\begin{array}{l}\text { Equipment } \\
\text { Condition }\end{array}$ & Commente \\
\hline 10.1 & 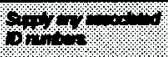 & 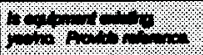 & $x_{1}+1,1+1$ & or & $1 \%+1 \%$ \\
\hline Lenk duction purp pt & $\begin{array}{l}\text { 241-Awo1c throuth - } \\
00 \mathrm{C}\end{array}$ & Yee lanl, wd & Yes [nd & $M$ & One ter ench terk \\
\hline Lovel sening srum (SpGWF wmp) & $\mathbf{M}$ & Yes [ent] & ran fud & S [di] & $m$ \\
\hline Gorma rad monitor (ump) & $m$ & $Y \in[m]$ & Nolud & $s[d+]$ & Dow not work on wy pil fudf \\
\hline Leak delocition sylum (pit) & M & $Y \in[m]$ & Yee lind & $5\{d\}$ & $m$ \\
\hline -Temperathre & $m$ & Yes [ant] & Yen not & $5(d 1)$ & m \\
\hline Sump & $M$ & No $(a n t)$ & $M$ & $m$ & M \\
\hline Drin & $m$ & Yes $(\operatorname{men}, \operatorname{and})$ & Yea & $M$ & Anumed apenatis \\
\hline fortable pit oproy & MA & $r e \tan \theta$ & $Y a$ & $M$ & Anumed operable \\
\hline Pump, moix & $M$ & Yes $(m e,=m a,=n$ & Nolind & M & Not hooked up. Onty hataled in AW101. Ind \\
\hline fump & NA & Yes $[m R,=m,=n]$ & No $\mathrm{Bnd}$ & M & ode hive lno \\
\hline Dy welle & MA & Yee [err]] & Yes pud & M & 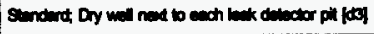 \\
\hline Condenesor pit & MA & M & M & $M$ & MA \\
\hline studp pemp pit & M & MA & M & $M$ & Ma \\
\hline sat wel puppt & M & $M$ & M & Ma & MA \\
\hline
\end{tabular}

NA = Not epplicable or not reqined $5 / 31 / 98$ 


\begin{tabular}{|c|c|c|c|c|c|}
\hline$w$ & [WPls & $\operatorname{low}=0$ & (on) 100 & w & (6) ent \\
\hline $\mathbf{w}$ & Itpls $\mathrm{s}$ & $|p m|=\lambda$ & pow $\infty x$ & w & woppop por \\
\hline 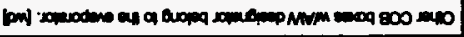 & w & pow $=0$ & {$[\mathrm{~m}]=0$} & $0 t^{\prime} 6 \pm-800$ & mog no now \\
\hline 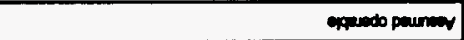 & (HP) & $\infty$ & $\operatorname{sen} 100$ & $w$ & 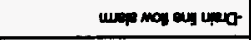 \\
\hline apado paun & |lpils $s$ & 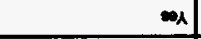 & soones 10 & $w$ & mpums anceory \\
\hline 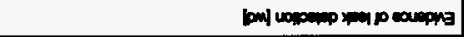 & $\mathbf{w}$ & w & $w$ & $w$ & $\operatorname{man}$ \\
\hline 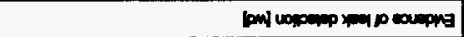 & $w$ & $w$ & $\mathbf{w}$ & $w$ & $\operatorname{arn} 75$ \\
\hline eppodo pounery & $\mathbf{w}$ & $\infty$ & beonel wa & w & on \\
\hline$w$ & $w$ & $w$ & Ionj on & w & ayconaw mot \\
\hline apop & $\mathbf{w}$ & {$[\mathrm{mow} \times \lambda$} & 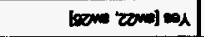 & $w$ & consopul uoneod enpen- \\
\hline somesermani & Ltopls & {$[p w]=\lambda$} & boven zow = & $w$ & 1000000 yor \\
\hline 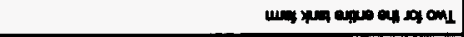 & $\mathbf{w}$ & {$[\mathrm{pm}] \operatorname{\infty } \alpha$} & $\mid \mathrm{pm}]=0 \mathrm{x}$ & g-peranutitz & at exs \\
\hline uopoevep yman & Lifis & 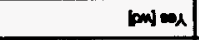 & benel wa & $w$ & 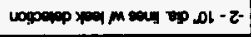 \\
\hline 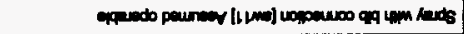 & w & $\infty$ & I I L & $w$ & hede wd exparad \\
\hline Domes am-on 1 & Ltpls & $\operatorname{pon} \mid \cos$ & lomed $\infty x$ & $w$ & volponep xpor \\
\hline 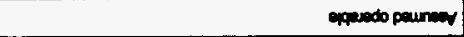 & $\mathbf{w}$ & $\infty$ & {$[a x=1$} & $w$ & war \\
\hline $200 \times 10100$ & $\mathbf{w}$ & 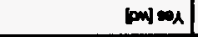 & 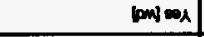 & COOMNLEC & Ant \\
\hline 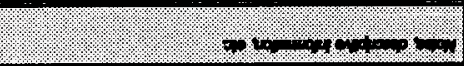 & 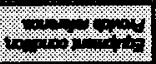 & 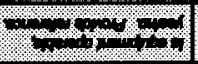 & Tropisy & 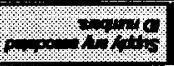 & $6=1$ \\
\hline 9aums & umpong & eqpusdo & remadounepg & Stis & מאומש \\
\hline
\end{tabular}




\begin{tabular}{|c|c|c|c|c|c|}
\hline 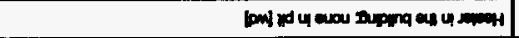 & $w$ & $p$ pwi $\infty_{1}$ & $|\operatorname{lom}| \infty \lambda$ & $w$ & 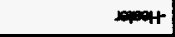 \\
\hline $\mathbf{w}$ & $w$ & $w$ & boul ow & wow & mavep pay dins - \\
\hline 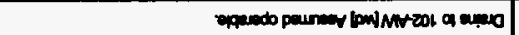 & $w$ & $\infty$ & $\operatorname{lon} \operatorname{sen} 100$ & $w$ & mat \\
\hline apudo peuriner| & $w$ & $\infty$ & In movel wa & w & dins- \\
\hline equado pounines & 【|p|s & $\infty$ & Ion sowed ax & w) & morno anopert \\
\hline equecos peunier| & 【Lpls $\mathrm{s}$ & $\infty$ & Ion 'toovel ax & $w$ & uoromep xper \\
\hline 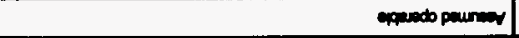 & $w$ & $\infty$ & 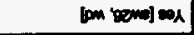 & w & mened moppose \\
\hline apencto pountar & 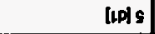 & $\approx$ & 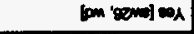 & $w$ & sonow part \\
\hline eppenco peunery & (tpls 5 & $\operatorname{\infty x}$ & Im 'gonel ax & $w$ & Denchan motr \\
\hline $\mathbf{w}$ & $\mathbf{w}$ & 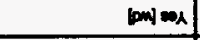 & $|p m|=\lambda$ & Loonntive & ad wopes \\
\hline$w$ & $w$ & $w$ & $w$ & $w$ & No ding waris \\
\hline on cooum oun kads & Ltpls & pow $\infty x$ & 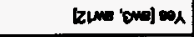 & w & volpoupo weor \\
\hline 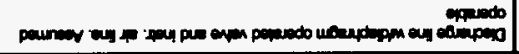 & w & $=0$ & [5med $\infty \mathrm{x}$ & $w$ & tert \\
\hline$w$ & $\mathbf{w}$ & {$[\mathrm{pm}]=0 \mathrm{e}$} & Iowed $\square \lambda$ & $w$ & 10w \\
\hline 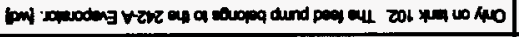 & w & 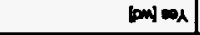 & 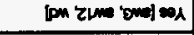 & 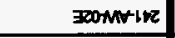 & ud dend pees \\
\hline 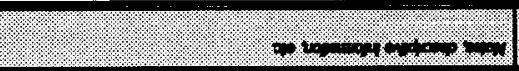 & 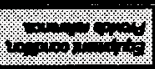 & . & 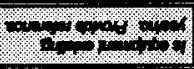 & 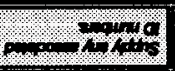 & 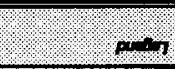 \\
\hline anumos & $\log _{\text {mation }}$ & eqpedo & 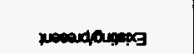 & 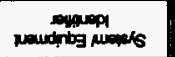 & ureats \\
\hline
\end{tabular}




\begin{tabular}{|c|c|c|c|c|c|}
\hline $\mathbf{w}$ & $w$ & $|\operatorname{low}|=0$ & {$[0 \times \mid \infty \lambda$} & LZZMNLIEZ & Aprea paunimin \\
\hline $\mathbf{w}$ & $\mathbf{N}$ & $w$ & [oul ow & W & 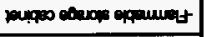 \\
\hline 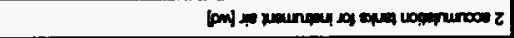 & $w$ & [on] $\operatorname{mex}$ & 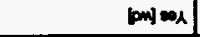 & $\mathbf{w}$ & aperappt \\
\hline$w$ & $(L P)<$ & | & $|p w| m \mid$ & loLMntre & Apres nosainos \\
\hline & & & & & apre \\
\hline 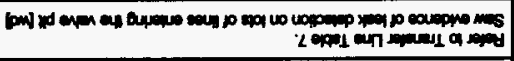 & w & $w$ & $w$ & $\mathbf{w}$ & soun \\
\hline & & & & & 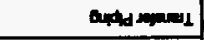 \\
\hline$w$ & L(p) $s$ & $w$ & {$[5001] \infty 1$} & $W$ & 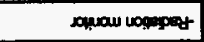 \\
\hline$w$ & $w$ & $w$ & Lowal on & W & severt \\
\hline$w$ & ILPIs & $m$ & bovel ow & $w$ & roupep por \\
\hline $\mathbf{w}$ & $w$ & $w$ & fow & $w$ & duns \\
\hline eqpeodo peuinarer & $w$ & $\infty$ & 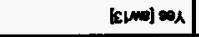 & $\mathbf{N}$ & una- \\
\hline 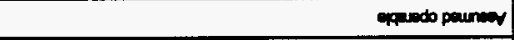 & ไاpls & $\infty$ & $\operatorname{sen} \theta \cos$ & $w$ & 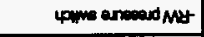 \\
\hline$w$ & Llpls & 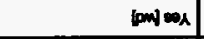 & Istmeid $\infty \lambda$ & $w$ & conpenop xor \\
\hline 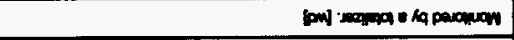 & (tpls $\varepsilon$ & 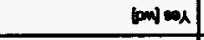 & boond $\infty \mathrm{x}$ & $w$ & andurow mas \\
\hline$w$ & $w$ & $\mid \mathrm{p} n \mathrm{y}=\mathrm{x}$ & $|p \times y|=0$ & Morte & dus \\
\hline 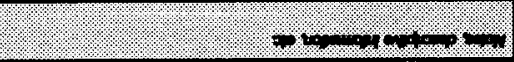 & 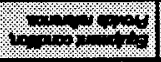 & $=20+1 \%$ & 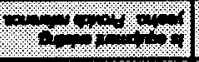 & 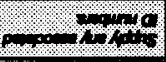 & $\infty$ \\
\hline 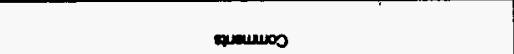 & uonpus & appedo & 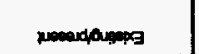 & 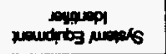 & womens \\
\hline
\end{tabular}




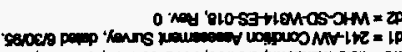

\begin{tabular}{|c|c|c|c|c|c|}
\hline 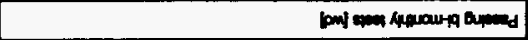 & $w$ & {$[\mathrm{p} \times] \times$} & 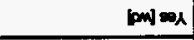 & w & 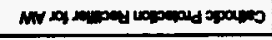 \\
\hline$w$ & $w$ & {$[\mathrm{p}) \infty \mathrm{N}$} & 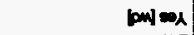 & w & 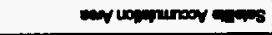 \\
\hline $\mathbf{w}$ & $w$ & $\operatorname{son}=0$ & $|\ln |=0$ & ZLEN+1k2 & 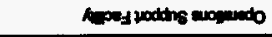 \\
\hline 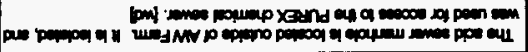 & $w$ & ow & 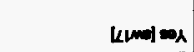 & w & 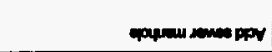 \\
\hline $\mathbf{w}$ & $\mathbf{w}$ & $|p+\infty| \infty \mid$ & $|\mathrm{pow}| \operatorname{sex}$ & w & on \\
\hline & & & & & 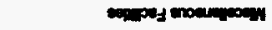 \\
\hline 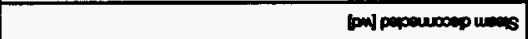 & $w$ & Inom ow & 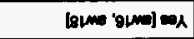 & $w$ & uopap din unas \\
\hline & & & & & Sח \\
\hline 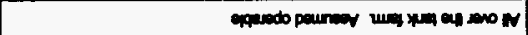 & $w$ & $\infty$ & $\mathrm{bow}=\mathrm{ex}$ & $w$ & cosen wor \\
\hline & & & & & 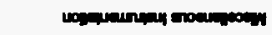 \\
\hline 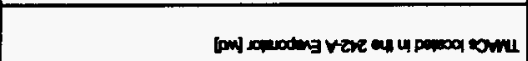 & [Lp] $\mathbf{s}$ & S & $\operatorname{low} \infty_{\alpha}$ & $\mathbf{w}$ & 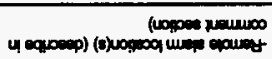 \\
\hline $\mathbf{w}$ & Lepis s] & $\operatorname{low} \mid=\lambda$ & $\tan ] \infty$ & $w$ & nos \\
\hline $\mathbf{m}$ & [Lpis s & {$[\mathrm{mon}]=0$} & 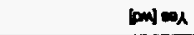 & $w$ & voppounoo ssvo \\
\hline $\mathbf{w}$ & [1:P]s & 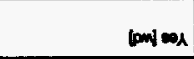 & $|p w| \omega_{\lambda}$ & w & 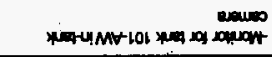 \\
\hline 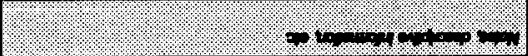 & $1 \%$ & 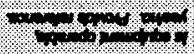 & 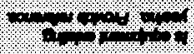 & 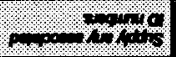 & 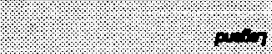 \\
\hline 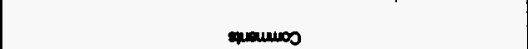 & uompus & onsodo & Memodounpeg & 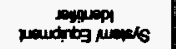 & unents \\
\hline
\end{tabular}


$m 0=1+2-605142$ Rov. 1

$m=H 140000011$ Rev 2

$m=12-7004011$ Rov. 2

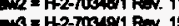

ans = H-2-70348/1 Pov. 15

and $1+2-70350 / 1$ Rov. 13.

ans = H-14-020000 sh1 Rov. 2

$106=$ H-1400000e-the Rov. 0

wh = H14000002 th3 Rov.

mo = H2-70112 Row. 4.

$=0=12-7041111$ Rov. 5

w10 = $142-70413$ Rev. 4.

cm1 = H2-70414 Row. 6

- M12 = H-270415 Rov. 5 .

M13 $=$ H2.70416 Row. 5.

aMA = 1-270101 Rov. 7 .

$\min =\mathrm{H}-2-705602$ Rov. 0

ans $=1-2-70000$ RN. 10

an17 = H2-70M00 Rer.

$\cos =4270 \mathrm{meg} \mathrm{R} \cdot \mathrm{s}$

- 79

जeO $=1+2-70607$

-nR1 $=142-70411 / 2$ Rov. 0

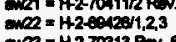

$023=1+2-70313$ Ror. 6

m24 = H+2-70402 Rov. 5

mes = $1+2-60006$ Rov. 4.

$m a b=1+2-7030 \%$, Rov. 2

mo7 = H-2-70350, Rev. 6

anes $=+1-200005$, Rov 7

ans = H-2-70315, Rov 0

arso $=1+2-70011, R$ Rov 0

and1 = H2-70118, Rev 2

ance $=1+2-70314$, Rov 0

ans3 = H2-70313, Rov 0

ans4 = H+270312 Rov 0

ans5 = H-2-T0310, Rov 0 
9/LS

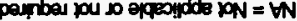

\begin{tabular}{|c|c|c|c|c|c|}
\hline $\mathbf{w}$ & 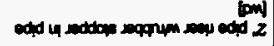 & $|p m| p s$ & ம人 & CIM & ad pod wes exply \\
\hline Inow ungaco pocos & 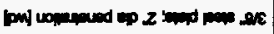 & |pw| & - & LXXM-LVE & od pod pes koupd \\
\hline Ipwi ungupos poos & 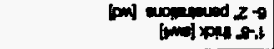 & (1) & $\boldsymbol{\oplus \boldsymbol { \Lambda }}$ & 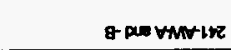 & aren \\
\hline 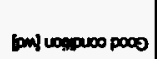 & 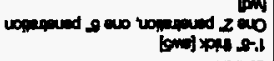 & 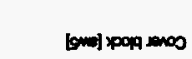 & $\omega$ & $000 \mathrm{n}+1 \mathrm{t} 2$ & Fon \\
\hline $\mathbf{w}$ & $\mathbf{w}$ & $\mathbf{W}$ & $\mathbf{w}$ & $\mathbf{W}$ & Fidund renins \\
\hline $\mathbf{w}$ & $\mathbf{w}$ & W & $\mathbf{w}$ & $\mathbf{W}$ & Fid dund aprs \\
\hline $\mathbf{w}$ & $\mathbf{w}$ & $\mathbf{w}$ & $\mathbf{w}$ & $w$ & id mampios \\
\hline 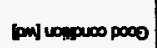 & 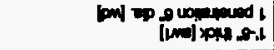 & [LNol Xpoq 2000 & ம & 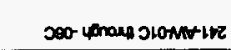 & 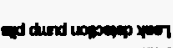 \\
\hline $\mathbf{w}$ & $\mathbf{w}$ & $\mathbf{w}$ & $\mathbf{w}$ & $\mathbf{w}$ & ad 1 ment \\
\hline $\mathbf{w}$ & m & $\mathbf{W}$ & $\mathbf{w}$ & $\mathbf{w}$ & Id expess \\
\hline $\mathbf{w}$ & w & $\mathbf{w}$ & $\mathbf{w}$ & $\mathbf{w}$ & 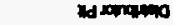 \\
\hline IpW] uogpuno poco & 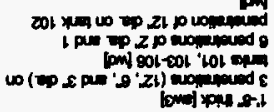 & tymel wap $=100$ & क人 & vo- Lnoup vountiz & andund pqus \\
\hline [pen usipuro poos & 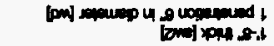 & {$[D n=]$ wop $=00$} & $\boldsymbol{\omega}$ & 800- yonare giontitz & addund wriey \\
\hline $\mathbf{W}$ & [LP] unas cuxp Y & mentips & $\boldsymbol{\omega} \lambda$ & SOL- Lonoun LOLtMth & 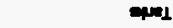 \\
\hline$m$ & 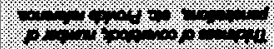 & 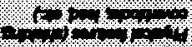 & 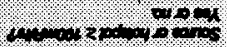 & 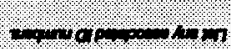 & $60+10,1$ \\
\hline 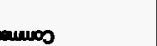 & 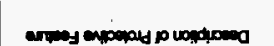 & anvey onponds & 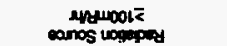 & al unats & wepers \\
\hline
\end{tabular}


95/ES penbel pou no eqpoydds poN = WN

\begin{tabular}{|c|c|c|c|c|c|}
\hline 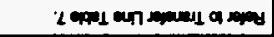 & $\mathbf{w}$ & W & $w$ & $\mathbf{w}$ & 1 \\
\hline $\mathbf{w}$ & $\mathbf{w}$ & $\mathbf{w}$ & $\mathbf{w}$ & $w$ & 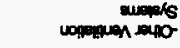 \\
\hline$w$ & 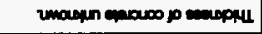 & 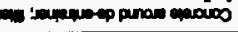 & $\boldsymbol{\infty}$ & 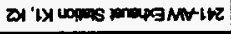 & 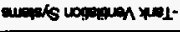 \\
\hline & & & & & WNH \\
\hline$w$ & $\mathbf{w}$ & $\mathbf{w}$ & $\mathbf{w}$ & $\mathbf{w}$ & odeq unens \\
\hline & & & & & unengusums \\
\hline 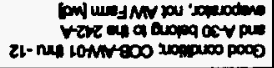 & 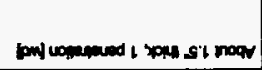 & 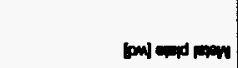 & $\boldsymbol{\omega} \boldsymbol{\lambda}$ & $01 M-800$ '60M-800 & manowor \\
\hline $\mathbf{W}$ & $\mathbf{w}$ & $\mathbf{W}$ & $w$ & W & 20 \\
\hline 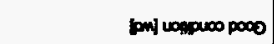 & 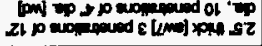 & 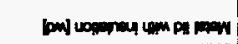 & ON & $M+1 / 2$ & a 4 4) \\
\hline $\mathbf{W}$ & $\mathbf{w}$ & 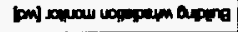 & कN & MULK & Pd expes \\
\hline $\mathbf{w}$ & 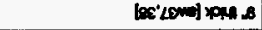 & $\operatorname{poph}=000$ & $\boldsymbol{\omega \lambda}$ & Eperez-1-dhan-ikz & 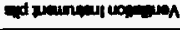 \\
\hline W & $\mathbf{w}$ & $\mathbf{w}$ & $\mathbf{w}$ & $\mathbf{w}$ & Folund wat \\
\hline $\mathbf{w}$ & 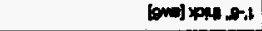 & bond $\times 909$ anos & $\boldsymbol{\omega}$ & $\exists 20 M N+1 K 2$ & ad dund paed \\
\hline $6: 1$ & 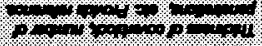 & 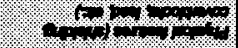 & 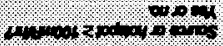 & 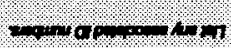 & $18000 \%=$ \\
\hline quanos & 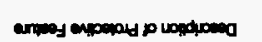 & arines exposald & 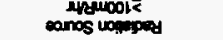 & 여 wents & unets \\
\hline
\end{tabular}




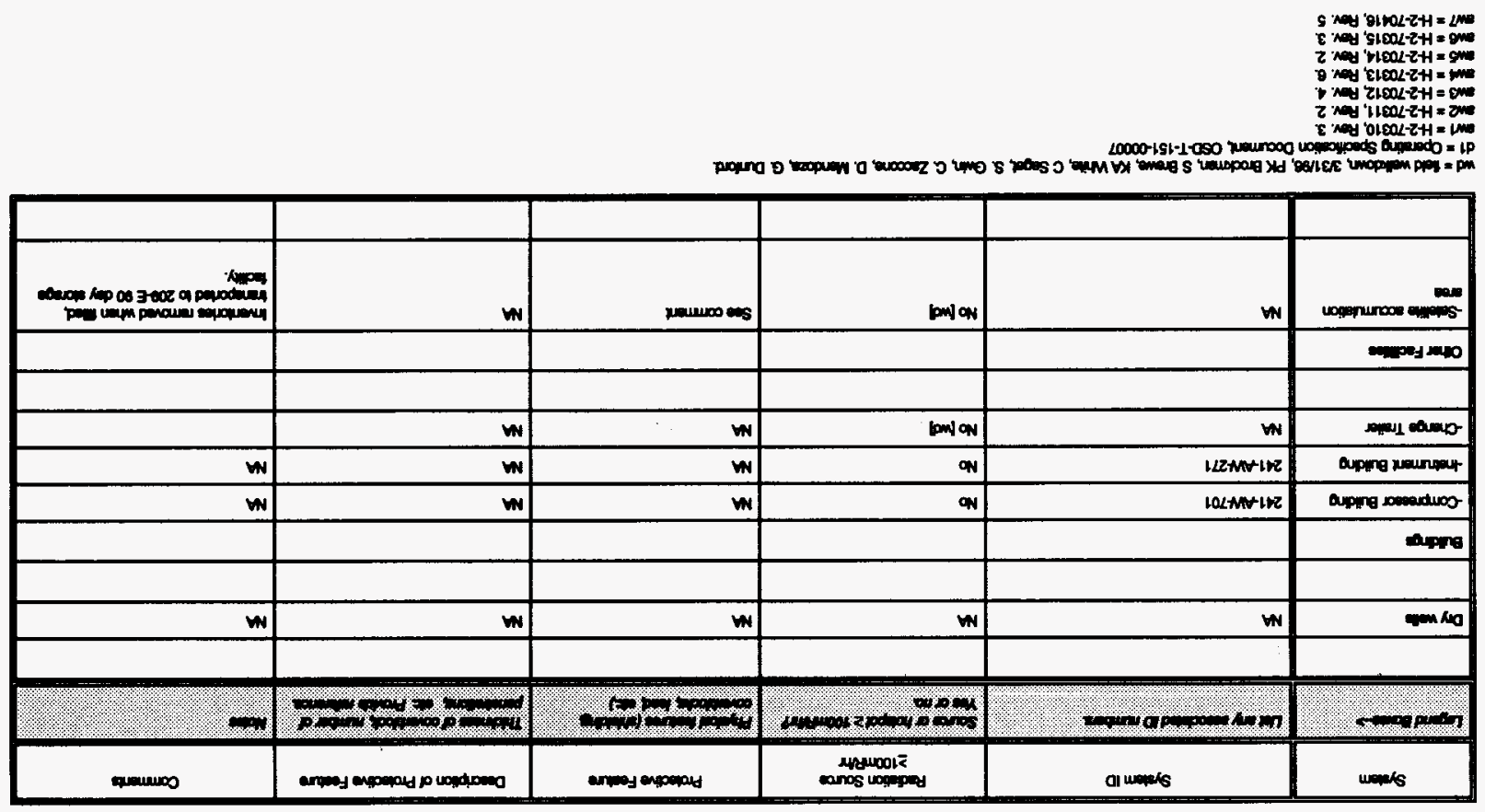


TABLE 6

Tank Farm or Miscellaneous Facility

AW

OSRUOSD SAFETY CONTROLS

\begin{tabular}{|c|c|c|c|c|c|c|c|}
\hline \multirow{2}{*}{$\begin{array}{l}\text { System/Structures/Components } \\
\text { Tank primary difi. pressure monftor }\end{array}$} & \multicolumn{3}{|c|}{ OSR Limit } & \multirow{2}{*}{$\frac{\text { OSD Limit }}{\text { NA }}$} & Implementing Procedures & Verification & \multirow[t]{2}{*}{ Comments } \\
\hline & WHC-SO-WM-OSR-016 & LCO & 3.3.1 & & $70-060-340$ & FV & \\
\hline Tanks 241-AW-101 through -106 & & NA & & NA & NA & NA & \\
\hline Tank Weste Instrumentation & & NA & & NA & NA & NA & \\
\hline Temperature probes (T.C Tree) & WHC-SD-WM-OSR-016 & SL & 2.2 & NA & $\begin{array}{l}\text { TF-OR-A-03 } \\
\text { TO-001-084 } \\
\text { TO-001-180 } \\
\text { TO-020-595 } \\
\text { TO-040-035 } \\
\text { TO-040-200 } \\
\text { TO-040-660 } \\
\text { TO-060-230 } \\
\text { TO-200-460 } \\
\text { TO-200-464 } \\
\text { TO-220-010 } \\
\text { TO-220-027 } \\
\text { TO-220-095 } \\
\text { TO-220-096 } \\
\text { TO-230-095 } \\
\text { TO-230-099 } \\
\text { TO-230-133 } \\
\text { TO-230-141 } \\
\text { TO-230-142 } \\
\text { TO-230-143 } \\
\text { TO-230-144 } \\
\text { TO-230-274 } \\
\text { TO-270-007 } \\
\text { TO-270-011 } \\
\text { TO-270-012 } \\
\text { TO-270-014 }\end{array}$ & FV OR & \\
\hline
\end{tabular}

\section{5/31/96}

NA = Not applicable or not required 
TABLE 6

Tank Farm or Miscellaneous Facility AW

OSR/OSD SAFETY CONTROLS

\begin{tabular}{|c|c|c|c|c|c|}
\hline System/Structures/Components & OSR Limit & OSD Limit & Implementing Procedures & Verification & Comments \\
\hline & WHC-SD-WM-OSR-016 SL 2.2 & NA & $\begin{array}{l}\text { TO-270-040 } \\
\text { TO-270-221 } \\
\text { TO-270-235 } \\
\text { TO-270-251 } \\
\text { TO-270-252 } \\
\text { TO-270-266 } \\
\text { TO-270-824 } \\
\text { TO-400-200 } \\
\text { TO-430-480 } \\
\text { TO-430-485 } \\
\text { TO-440-002 }\end{array}$ & FV DR & \\
\hline & WHC-SD-WM-OSA-016 LCO 3.2 .1 & +2 & NA & FV & $\begin{array}{l}\text { LCO-Not Implemented by } \\
\text { ECN }\end{array}$ \\
\hline & WHC-SD-WM-OSR-016 LCO 3.2 .2 & NA & NA & NA & ECN 619396 \\
\hline & $\begin{array}{lll}\text { WHC-SD-WM-OSR-016 } & \text { SA } & 3.2 .2 .1 \\
\end{array}$ & 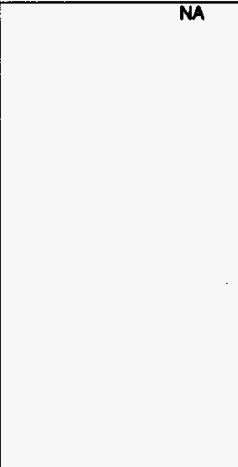 & $\begin{array}{l}\text { TF-OR-A-03 } \\
\text { TO-001-184 } \\
\text { TO-040-660 } \\
\text { TO-220-095 } \\
\text { TO-220-096 } \\
\text { TO-230-095 } \\
\text { TO-230-099 } \\
\text { TO-230-133 } \\
\text { TO-230-141 } \\
\text { TO-230-142 } \\
\text { TO-230-143 } \\
\text { TO-230-144 } \\
\text { TO-270-007 } \\
\text { TO-270-011 } \\
\text { TO-270-012 } \\
\text { TO-270-035 } \\
\text { TO-270-040 } \\
\text { T0-400-200 } \\
\text { TO-430-480 } \\
\text { T0-430-485 } \\
\text { TO-440-002 }\end{array}$ & FV DR & ECN 619398 \\
\hline & NA & OSD-T-151-00007 7.2.6.a & T0-001-182 & NA & \\
\hline
\end{tabular}

\section{$05 / 31 / 96$}

NA $=$ Not applicable or not required 
TABLE 6

Tank Farm or Miscellaneous Facility AW

\section{OSROSD SAFETY CONTROLS}

\begin{tabular}{|c|c|c|c|c|c|}
\hline \multirow{3}{*}{ System/Structures/Components } & OSR Limit & OSD Limit & Implementing Procedures & Verification & Comments \\
\hline & NA & OSD-T-151-00007 7.2.6.8 & $\begin{array}{l}\text { TO-001-184 } \\
\text { TO-040-760 } \\
\text { TO-200-010 } \\
\text { TO-220-095 } \\
\text { TO-230-095 } \\
\text { TO-230-133 } \\
\text { TO-230-274 } \\
\text { TO-270-040 } \\
\text { TO-270-204 } \\
\text { TO-270-221 } \\
\text { TO-270-236 } \\
\text { TO-270-252 } \\
\text { TO-270-266 } \\
\text { TO-270-826 } \\
\text { TO-270-828 } \\
\text { TO-400-200 } \\
\text { TO-430-480 } \\
\text { TO-600-080 }\end{array}$ & $\mathrm{NA}$ & \\
\hline & NA & OSD-T-151-00007 7.2.6.b & $\begin{array}{l}\text { TO-001-182 } \\
\text { TO-001-184 } \\
\text { TO-040-760 } \\
\text { TO-200-010 } \\
\text { TO-220-085 } \\
\text { TO-230-095 } \\
\text { TO-230-133 } \\
\text { TO-230-274 } \\
\text { TO-270-040 } \\
\text { TO-270-204 } \\
\text { TO-270-221 } \\
\text { TO-270-236 } \\
\text { TO-270-252 } \\
\text { TO-270-266 } \\
\text { TO-270-826 } \\
\text { TO-270-828 } \\
\text { TO-400-200 } \\
\text { TO-430-480 } \\
\text { TO-600-080 }\end{array}$ & NA & \\
\hline & MA & OSD-T-151-00007 7.2.6.c & TO-001-182 & NA & \\
\hline
\end{tabular}

$05 / 31 / 96$

NA $=$ Not applicable or not required 
TABLE 6

Tank Farm or Miscellaneous Facility AW

\section{OSR/OSD SAFETY CONTROLS}

\begin{tabular}{|c|c|c|c|c|c|}
\hline System/Structures/Components & OSR Limit & OSD Limit & Implementing Procedures & Verification & Comments \\
\hline & NA & OSD-T-151-00007 7.2.6.c & $\begin{array}{l}\text { TO-001-184 } \\
\text { TO-040-760 } \\
\text { TO-200-010 } \\
\text { TO-220-095 } \\
\text { TO-230-095 } \\
\text { TO-230-133 } \\
\text { TO-230-274 } \\
\text { TO-270-040 } \\
\text { TO-270-204 } \\
\text { TO-270-221 } \\
\text { TO-270-236 } \\
\text { TO-270-252 } \\
\text { TO-270-266 } \\
\text { TO-270-828 } \\
\text { TO-270-828 } \\
\text { TO-400-200 } \\
\text { TO-430-480 } \\
\text { TO-600-080 }\end{array}$ & NA & \\
\hline Lquid level (Primary) & WHC-SD-WM-OSR-016 SL 2.1 & NA & $\begin{array}{l}\text { TF-OR-A-03 } \\
\text { TO-020-330 } \\
\text { TO-020-820 } \\
\text { T0-040-200 } \\
\text { TO-060-230 } \\
\text { TO-060-340 } \\
\text { TO-220-019 } \\
\text { TO-220-095 } \\
\text { TO-290-095 } \\
\text { TO-230-099 } \\
\text { TO-230-133 } \\
\text { TO-230-142 } \\
\text { TO-230-143 } \\
\text { TO-230-274 } \\
\text { TO-440-002 }\end{array}$ & FV DR & \\
\hline & WHC-SD-WM-OSR-016 LCO 3.1 .2 & NA & $\begin{array}{l}\text { ARP-T-231-00102 } \\
\text { ARP-T-231-00108 } \\
\text { ARP-T-231-00105 } \\
\text { ARP-T-231-00106 }\end{array}$ & NA & \\
\hline
\end{tabular}

05/31/96

NA $=$ Not applicable or not required 
TABLE 6

Tank Farm or Miscellaneous Facility AW

\section{OSR/OSD SAFETY CONTROLS}

\begin{tabular}{|c|c|c|c|c|c|}
\hline System/Structures/Components & OSR Limit & OSD Limit & Implementing Procedures & Verification & Comments \\
\hline & WHC-SD-WM-OSR-016 LCO 3.1 .2 & NA & $\begin{array}{l}\text { ARP-T-271-00101 } \\
\text { ARP-T-271-00102 } \\
\text { ARP-T-271-00103 } \\
\text { ARP-T-271-00104 } \\
\text { ARP-T-271-00105 } \\
\text { ARP-T-271-00106 } \\
\text { ARP-T-271-00107 } \\
\text { ARP-T-271-00108 } \\
\text { TF-OR-A-03 } \\
\text { TO-001-084 } \\
\text { TO-001-180 } \\
\text { TO-001-184 } \\
\text { TO-020-025 } \\
\text { TO-020-330 } \\
\text { TO-020-595 } \\
\text { TO-020-820 } \\
\text { TO-040-200 } \\
\text { TO-080-106 } \\
\text { TO-080-230 } \\
\text { TO-080-340 } \\
\text { TO-200-460 } \\
\text { TO-220-027 } \\
\text { TO-220-095 } \\
\text { TO-220-096 } \\
\text { TO-230-095 } \\
\text { TO-230-099 } \\
\text { TO-230-133 } \\
\text { TO-230-142 } \\
\text { TO-230-143 } \\
\text { TO-230-144 } \\
\text { TO-230-274 } \\
\text { TO-270-007 } \\
\text { TO-270-012 } \\
\text { TO-270-014 } \\
\text { TO-270-235 } \\
\text { TO-270-251 } \\
\text { TO-270-252 } \\
\text { TO-270-824 } \\
\text { TO-400-200 } \\
\text { TO-430-480 }\end{array}$ & NA & \\
\hline
\end{tabular}

\section{$05 / 31 / 96$}

$\mathrm{NA}=$ Not applicable or not required 
TABLE 6

Tank Farm or Miscellaneous Facility

AW

OSR/OSD SAFETY CONTROLS

\begin{tabular}{|c|c|c|c|c|c|c|c|}
\hline \multirow{3}{*}{ System/Structures/Components } & \multicolumn{2}{|c|}{ OSR Limit } & \multicolumn{2}{|c|}{ OSD Limit } & \multirow{2}{*}{$\begin{array}{l}\text { Implementing Procedures } \\
\text { TO-430-485 } \\
\text { TO-440-002 }\end{array}$} & \multirow{2}{*}{$\frac{\text { Verification }}{N A}$} & \multirow[t]{2}{*}{ Comments } \\
\hline & WHC-SD-WM-OSR-016 & LCO 3.1 .2 & & NA & & & \\
\hline & WHC-SD-WM-SAR-016 & 11.5 & OSO-T-151-00007 & 7.2.2.8 & $\begin{array}{l}\text { TF-OA-A-03 } \\
\text { TO-OO1-182 } \\
\text { TO-020-240 } \\
\text { TO-020-420 } \\
\text { TO-020-820 } \\
\text { TO-040-580 } \\
\text { TO-230-095 } \\
\text { TO-230-274 } \\
\text { TO-270-204 } \\
\text { TO-270-221 } \\
\text { TO-270-238 } \\
\text { TO-270-252 } \\
\text { TO-270-268 } \\
\text { TO-270-828 } \\
\text { TO-270-828 }\end{array}$ & NA & \\
\hline Lquild level (Bectap) & WHC-SD-WM-SAR-016 & 11.5 & OSD-T-151-00007 & 7.2.2.a & $\begin{array}{l}\text { TF-OA-A-03 } \\
\text { TO-001-182 } \\
\text { TO-020-240 } \\
\text { TO-020-2020 } \\
\text { TO-020-820 } \\
\text { TO-040-580 } \\
\text { TO-230-095 } \\
\text { TO-230-274 } \\
\text { TO-270-204 } \\
\text { TO-270-221 } \\
\text { TO-270-236 } \\
\text { TO-270-252 } \\
\text { TO-270-266 } \\
\text { TO-270-826 } \\
\text { TO-270-828 }\end{array}$ & NA & \\
\hline \multirow[t]{2}{*}{ High level probe } & \multicolumn{2}{|r|}{ NA } & & NA & MA & NA & \\
\hline & WHC-SD-WM-OSR-016 & $\begin{array}{ll}\text { SR } & 3.1 .2 .1 \\
\end{array}$ & & NA & TF-OA-A-03 & NA & \\
\hline
\end{tabular}

05/31/96

NA $=$ Not applicable or not required 
TABLE 6

Tank Farm or Miscellaneous Facility AW

OSR/OSD SAFETY CONTROLS

\begin{tabular}{|c|c|c|c|c|c|c|}
\hline \multirow{2}{*}{ System/Structures/Components } & \multicolumn{2}{|c|}{ OSR Limit } & \multirow{2}{*}{$\frac{\text { OSD Limit }}{\text { NA }}$} & \multirow[b]{2}{*}{$\begin{array}{l}\text { Implementing Procedures } \\
\text { TO-001-084 } \\
\text { TO-001-180 } \\
\text { TO-001-184 } \\
\text { TO-020-025 } \\
\text { TO-020-330 } \\
\text { TO-020-595 } \\
\text { TO-020-820 } \\
\text { TO-040-200 } \\
\text { TO-060-340 } \\
\text { TO-200-400 } \\
\text { TO-200-460 } \\
\text { TO-220-095 } \\
\text { TO-220-096 } \\
\text { TO-230-085 } \\
\text { TO-230-099 } \\
\text { TO-230-133 } \\
\text { TO-230-141 } \\
\text { TO-230-142 } \\
\text { TO-230-143 } \\
\text { TO-230-144 } \\
\text { TO-270-007 } \\
\text { TO-270-012 } \\
\text { TO-270-014 } \\
\text { TO-270-235 } \\
\text { TO-270-251 } \\
\text { TO-270-252 } \\
\text { TO-400-200 } \\
\text { TO-430-480 } \\
\text { TO-430-485 } \\
\text { TO-440-002 }\end{array}$} & \multirow{2}{*}{$\frac{\text { Verification }}{\mathrm{NA}}$} & Comments \\
\hline & WHC-SD-WM-OSR-016 SA & 3.1 .2 .1 & & & & \\
\hline Tank primary ciff. pressure monitor & WHC-SD-WM-OSA-016 SL & 2.3 & NA & $\begin{array}{l}\text { TF-FT-239-001 } \\
\text { TF-FT-279-001 } \\
\text { TO-040-035 } \\
\text { TO-060-101 } \\
\text { TO-060-230 } \\
\text { TO-060-340 } \\
\text { TO-230-095 } \\
\text { TO-230-133 }\end{array}$ & FV DR & \\
\hline
\end{tabular}

05/31/96

NA = Not applicable or not required 
TABLE 6

Tank Farm or Miscellaneous Facility

AW

OSRIOSD SAFETY CONTROLS

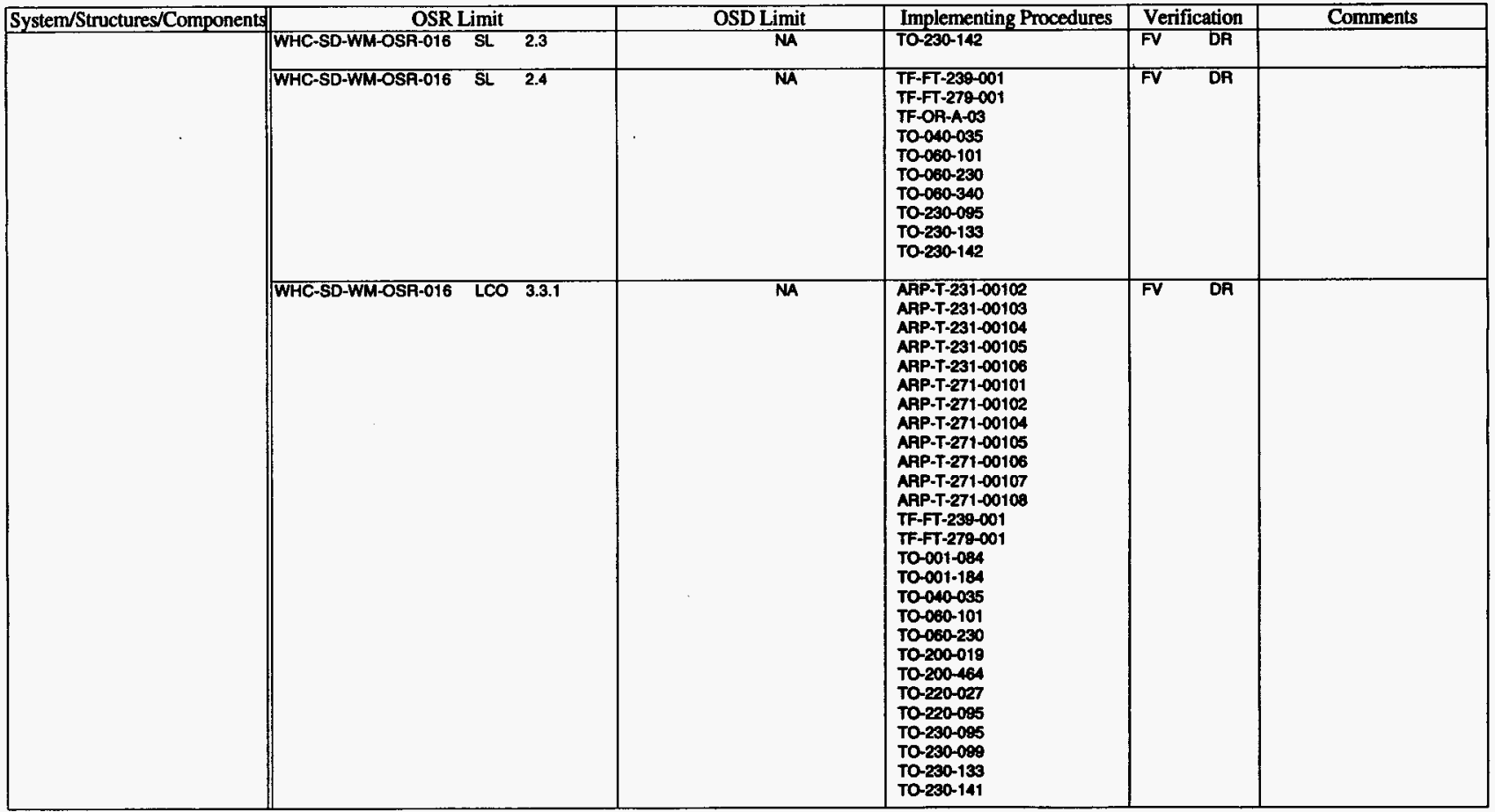

05/31/96

NA $=$ Not applicable or not required 
TABLE 6

Tank Farm or Miscellaneous Facility AW

\section{OSR/OSD SAFETY CONTROLS}

\begin{tabular}{|c|c|c|c|c|c|}
\hline System/Structures/Components & OSR Limit & OSD Limit & Implementing Procedures & Verification & Comments \\
\hline 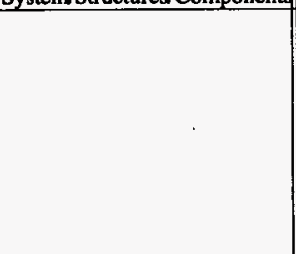 & WHC-SD-WM-OSA-016 LCO 3.3 .1 & NA & $\begin{array}{l}\text { TO-230-142 } \\
\text { TO-230-143 } \\
\text { TO-230-144 } \\
\text { TO-230-274 } \\
\text { TO-270-007 } \\
\text { TO-270-011 } \\
\text { TO-270-012 } \\
\text { TO-270-235 } \\
\text { TO-270-824 } \\
\text { TO-400-200 } \\
\text { TO-430-480 }\end{array}$ & FV DR & \\
\hline & 更 & +3 & $\begin{array}{l}\text { AAP-T-231-00101 } \\
\text { AAP-T-231-00105 } \\
\text { ARP-T-231-00106 } \\
\text { ARP-T-271-00102 } \\
\text { ARP-T-271-00103 } \\
\text { ARP-T-271-00104 } \\
\text { AAP-T-271-00105 } \\
\text { ARP-T-271-00106 } \\
\text { ARP-T-271-00107 } \\
\text { AAP-T-271-00108 } \\
\text { ARP-T }-601-00295 \\
\text { TF-OR-A-03 } \\
\text { TO-040-035 } \\
\text { TO-060-101 } \\
\text { TO-060-230 } \\
\text { TO-060-340 } \\
\text { TO-230-095 } \\
\text { TO-230-133 } \\
\text { TO-230-142 }\end{array}$ & F & \\
\hline & WHC-SD-WM-OSR-016 SA 3.3.2.1 & - & $\begin{array}{l}\text { AAP-T-231-00101 } \\
\text { TF-OR-A-03 } \\
\text { TO-040-035 } \\
\text { TO-060-101 } \\
\text { TO-060-340 }\end{array}$ & FV $\quad$ DR & \\
\hline & $\begin{array}{ll}\text { WHC-SD-WM-SAR-016 } & 11.6\end{array}$ & OSD-T-151-00007 7.2 .5 & $\begin{array}{l}\text { TO-060-105 } \\
\text { T0-060-341 }\end{array}$ & NA & \\
\hline
\end{tabular}

$05 / 31 / 96$

NA = Not applicable or not required 
TABLE 6

Tank Farm or Miscellaneous Facility

AW

OSR/OSD SAFETY CONTROLS

\begin{tabular}{|c|c|c|c|c|c|c|c|}
\hline \multirow[t]{2}{*}{ System/Structures/Components } & \multicolumn{2}{|c|}{ OSR Limit } & \multicolumn{2}{|c|}{ OSD Limit } & \multirow{2}{*}{$\begin{array}{l}\text { Implementing Procedures } \\
\text { T0-400-245 }\end{array}$} & \multirow{2}{*}{$\frac{\text { Verification }}{\mathrm{NA}}$} & \multirow[t]{2}{*}{ Comments } \\
\hline & WHC-SD-WM-SAR-016 & 11.6 & OSD-T-151-00007 & 7.2 .5 & & & \\
\hline Ges monltoring / SHMS & & & & NA & NA & NA & \\
\hline Pressure & & & & NA & NA & MA & \\
\hline Tank Annulus Instrumentation & & & & NA & NA & NA & \\
\hline $\begin{array}{l}\text { Leak detection manual tape } \\
\text { (conductivity probe) }\end{array}$ & WHC-SD-WM-SAR-016 & 11.4 & OSD-T-151-00007 & 7.2 .9 & $\begin{array}{l}\text { ARP-T-271-00101 } \\
\text { ARP-T-271-00102 } \\
\text { ARP-T-271-00103 } \\
\text { ARP-T-271-00104 } \\
\text { ARP-T-271-00105 } \\
\text { ARP-T-271-00106 } \\
\text { ARP-T-271-00107 } \\
\text { ARP-T-271-00108 } \\
\text { TO-001-184 } \\
\text { TO-020-210 } \\
\text { TO-040-590 } \\
\text { TO-060-185 } \\
\text { TO-060-240 } \\
\text { TO-080-341 } \\
\text { TO-220-095 } \\
\text { TO-230-098 } \\
\text { TO-230-133 } \\
\text { TO-230-274 } \\
\text { TO-270-040 } \\
\text { TO-400-200 } \\
\text { TO-430-480 }\end{array}$ & NA & \\
\hline CAM Rad Monitor & WHC-SD-WM-SAR-016 & 11.4 & OSD-T-151-00007 & 7.2 .9 & $\begin{array}{l}\text { ARP-T-271-00101 } \\
\text { ARP-T-271-00102 } \\
\text { ARP-T-271-00103 } \\
\text { ARP-T-271-00104 }\end{array}$ & $\mathbf{N A}$ & \\
\hline
\end{tabular}

$05 / 31 / 96$

$\mathrm{NA}=$ Not applicable or not required

B-159 
TABLE 6

Tank Farm or Miscellaneous Facility AW

OSR/OSD SAFETY CONTROLS

\begin{tabular}{|c|c|c|c|c|c|c|}
\hline System/Structures/Components & OSR & & OSD Limit & Implementing Procedures & Verification & Comments \\
\hline o 20 & WHC-SD-WM-SAR-016 & 11.4 & OSD-T-15:-00007 7.2 .9 & $\begin{array}{l}\text { ARP-T-271-00105 } \\
\text { ARP-T-271-00106 } \\
\text { ARP-T-271-00107 } \\
\text { ARP-T-271-00108 } \\
\text { TO-001-184 } \\
\text { TO-020-210 } \\
\text { TO-040-590 } \\
\text { TO-060-185 } \\
\text { TO-060-240 } \\
\text { TO-060-341 } \\
\text { TO-220-095 } \\
\text { TO-230-099 } \\
\text { TO-230-133 } \\
\text { TO-230-274 } \\
\text { TO-270-040 } \\
\text { TO-400-200 } \\
\text { TO-430-480 }\end{array}$ & NA & \\
\hline Pump pit leak detection & |WHC-SD-WM-SAR-016 & 11.7 & OSD-T-151-00007 $\quad 7.2 .10$ & $\begin{array}{l}\text { TO-001-184 } \\
\text { TO-220-085 } \\
\text { TO-230-099 } \\
\text { TO-230-133 } \\
\text { TO-230-274 } \\
\text { TO-270-252 } \\
\text { TO-400-200 } \\
\text { TO-430-480 } \\
\text { TO- } 450-150\end{array}$ & NA & \\
\hline $\begin{array}{l}\text { Laek datection conductivity (manual } \\
\text { tape) }\end{array}$ & WHC-SD-WM-SAR-016 & 11.7 & OSD-T-151-00007 $\quad 7.2 .10$ & $\begin{array}{l}\text { TO-001-184 } \\
\text { TO-220-095 } \\
\text { TO-230-099 } \\
\text { TO-230-133 } \\
\text { TO-230-274 } \\
\text { TO-270-252 } \\
\text { TO-400-200 } \\
\text { TO-430-480 }\end{array}$ & MA & \\
\hline
\end{tabular}

05/31/96

$\mathrm{NA}=$ Not applicable or not required 
TABLE 6

Tank Farm or Miscellaneous Facility

AW

OSR/OSD SAFETY CONTROLS

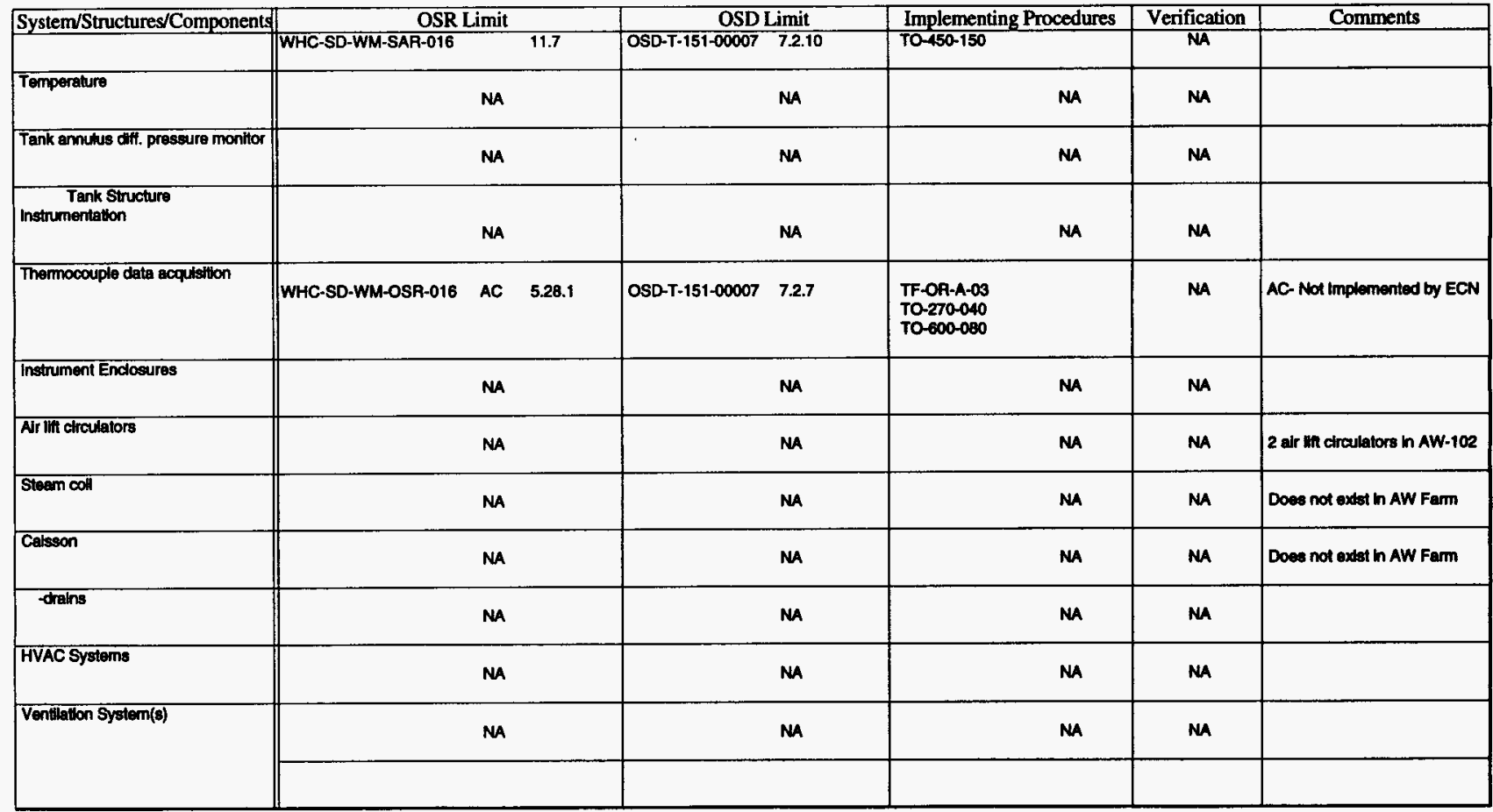

05/31/96

$\mathrm{NA}=\mathrm{Not}$ applicable or not required 
TABLE 6

Tank Farm or Miscellaneous Facility

AW

OSROSD SAFETY CONTROLS

\begin{tabular}{|c|c|c|c|c|c|}
\hline System/Structures/Components & OSR Limit & OSD Limit & Implementing Procedures & Verification & Comments \\
\hline Passtvo & NA & NA & NA & NA & \\
\hline \multirow[t]{2}{*}{-Active } & NA & NA & NA & NA & \\
\hline & NA & OSD-T-151-00007 7.3 .2 & $\begin{array}{l}\text { TO-001-182 } \\
\text { TO-001-184 } \\
\text { TO-020-155 } \\
\text { TO-020-925 } \\
\text { TO-060-230 } \\
\text { TO-060-340 } \\
\text { TO-060-403 } \\
\text { TO-080-500 } \\
\text { TO-060-503 } \\
\text { TO-060-516 } \\
\text { TO-230-095 } \\
\text { TO-230-133 } \\
\text { TO-230-274 } \\
\text { TO-270-204 } \\
\text { TO-270-221 } \\
\text { TO-270-236 } \\
\text { TO-270-252 } \\
\text { TO-270-266 } \\
\text { TO-270-626 } \\
\text { TO-270-828 }\end{array}$ & NA & \\
\hline $\begin{array}{l}\text { Shared/common vent system? } \\
\text { (Describe in comments section) }\end{array}$ & NA & NA & NA & NA & \\
\hline Exhaust venthatchway & NA & NA & NA & NA & \\
\hline Annulus air lntake assembites & NA & NA & NA & NA & \\
\hline -Primary alr Intake assemblies & NA & NA & NA & NA & \\
\hline
\end{tabular}

$05 / 31 / 96$

NA $=$ Not applicable or not required 


\begin{tabular}{|c|c|c|c|c|c|c|}
\hline \multirow{2}{*}{\begin{tabular}{|c|} 
System/Structures/Components \\
-Pro-fiters (yes $/ \mathrm{no})$
\end{tabular}} & OSR Limit & \multicolumn{2}{|c|}{ OSD Limit } & Implementing Procedures & \multirow{2}{*}{$\frac{\text { Verification }}{\mathrm{NA}}$} & \multirow[t]{2}{*}{ Comments } \\
\hline & NA & & NA & NA & & \\
\hline $\begin{array}{l}\text { HEPA fitters (how many fitters, } \\
\text { stages, parailel vs serles etc.) }\end{array}$ & NA & & NA & NA & NA & \\
\hline -Fans & NA & & NA & NA & NA & \\
\hline Do-entrainers & NA & & NA & NA & NA & \\
\hline Heaters & NA & & NA & NA & NA & \\
\hline HVAC Instrumentation & NA & & NA & NA & MA & \\
\hline & MA & OSD-T-151-00007 & 7.3.1.B & $\begin{array}{l}\text { TO-060-105 } \\
\text { TO-060-230 } \\
\text { TO-060-240 } \\
\text { TO-060-340 } \\
\text { TO-060-341 }\end{array}$ & NA & \\
\hline Main stack monttor & NA & OSD-T-15t-00007 & 7.3.1.D & TO-060-240 & NA & \\
\hline & NA & OSD-T-151-00031 & 31.2 .2 .2 & TO-040-590 & NA & \\
\hline Stack montior -tor annudus systom & NA & & NA & NA & NA & \\
\hline Primary stack pressure & NA & & NA & NA & MA & \\
\hline Annulus stack pressure & NA & & NA & M & Ma & \\
\hline
\end{tabular}

05/31/96

NA $=$ Not applicable or not required 
WHC-SD-WM-HIE-007

REV 0

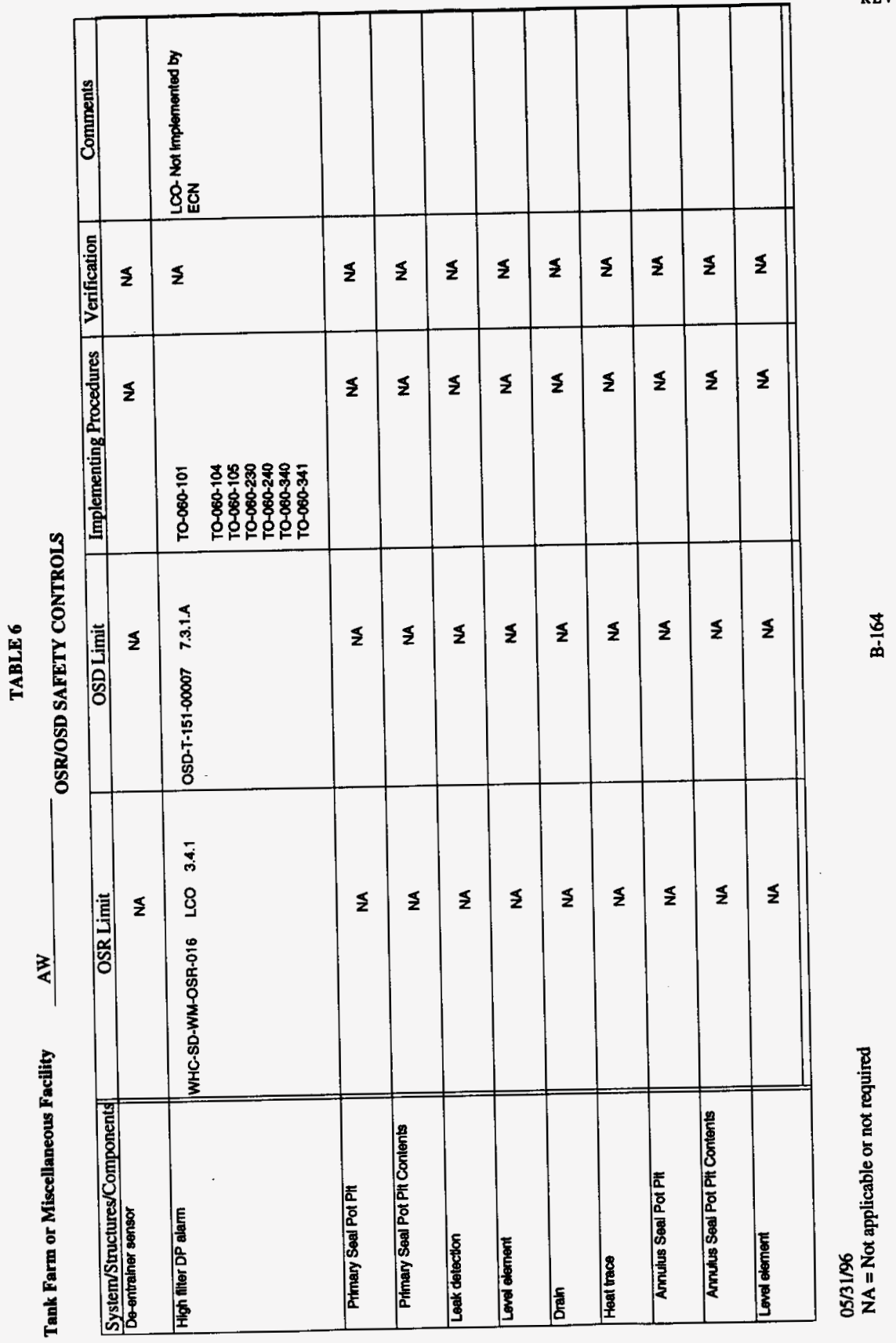


Tank Farm or Miscellaneous Facility

TABLE 6

AW

OSR/OSD SAFETY CONTROLS

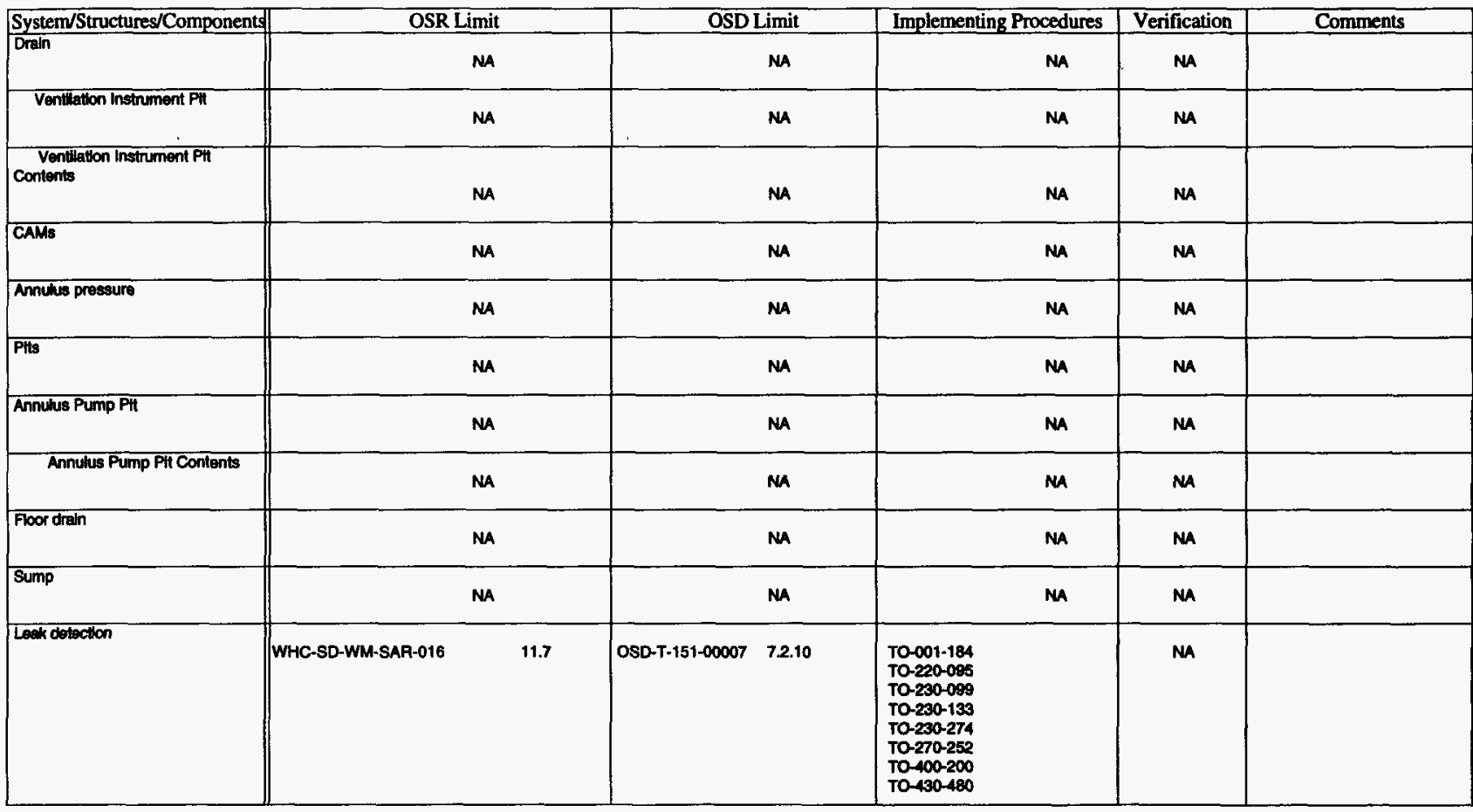

05/31/96

NA $=$ Not applicable or not required 
TABLE 6

Tank Farm or Miscellaneous Facility

AW

OSR/OSD SAFETY CONTROLS

\begin{tabular}{|c|c|c|c|c|c|c|}
\hline System/Structures/Components & OSR I & & OSD Limit & Implementing Procedures & Verification & Comments \\
\hline & WHC-SD-WM-SAR-016 & 11.7 & OSD-T-151-00007 $\quad 7.2 .10$ & T0-450-150 & NA & \\
\hline Portable ph spray & & & NA & NA & NA & \\
\hline Pump motor & & & NA & NA & NA & \\
\hline Central Pump Pit & & & NA & NA & NA & \\
\hline Central Pump PIt Conlents & & & NA & NA & NA & \\
\hline Leak detection & WHC-SD-WM-SAR-016 & 11.7 & OSD-T-151-00007 $\quad 7.2 .10$ & $\begin{array}{l}\text { TO-001-184 } \\
\text { TO-220-095 } \\
\text { TO-230-099 } \\
\text { TO-230-133 } \\
\text { TO-230-274 } \\
\text { TO-270-252 } \\
\text { TO-400-200 } \\
\text { TO-430-480 } \\
\text { TO-450-150 }\end{array}$ & NA & \\
\hline Floor drein & & & NA & NA & NA & \\
\hline Portable plt spray & & & NA & NA & NA & \\
\hline Pump Motor & & & NA & NA & NA & \\
\hline Siuny distributor & & & NA & NA & NA & \\
\hline Distilbutor Pit & & & NA & $\mathbf{M}$ & NA & Does not exdat in AW Fam \\
\hline
\end{tabular}

$05 / 31 / 96$

NA $=$ Not applicable or not required

B-166 
TABLE 6

Tank Farm or Miscellaneous Facility

AW

OSR/OSD SAFETY CONTROLS

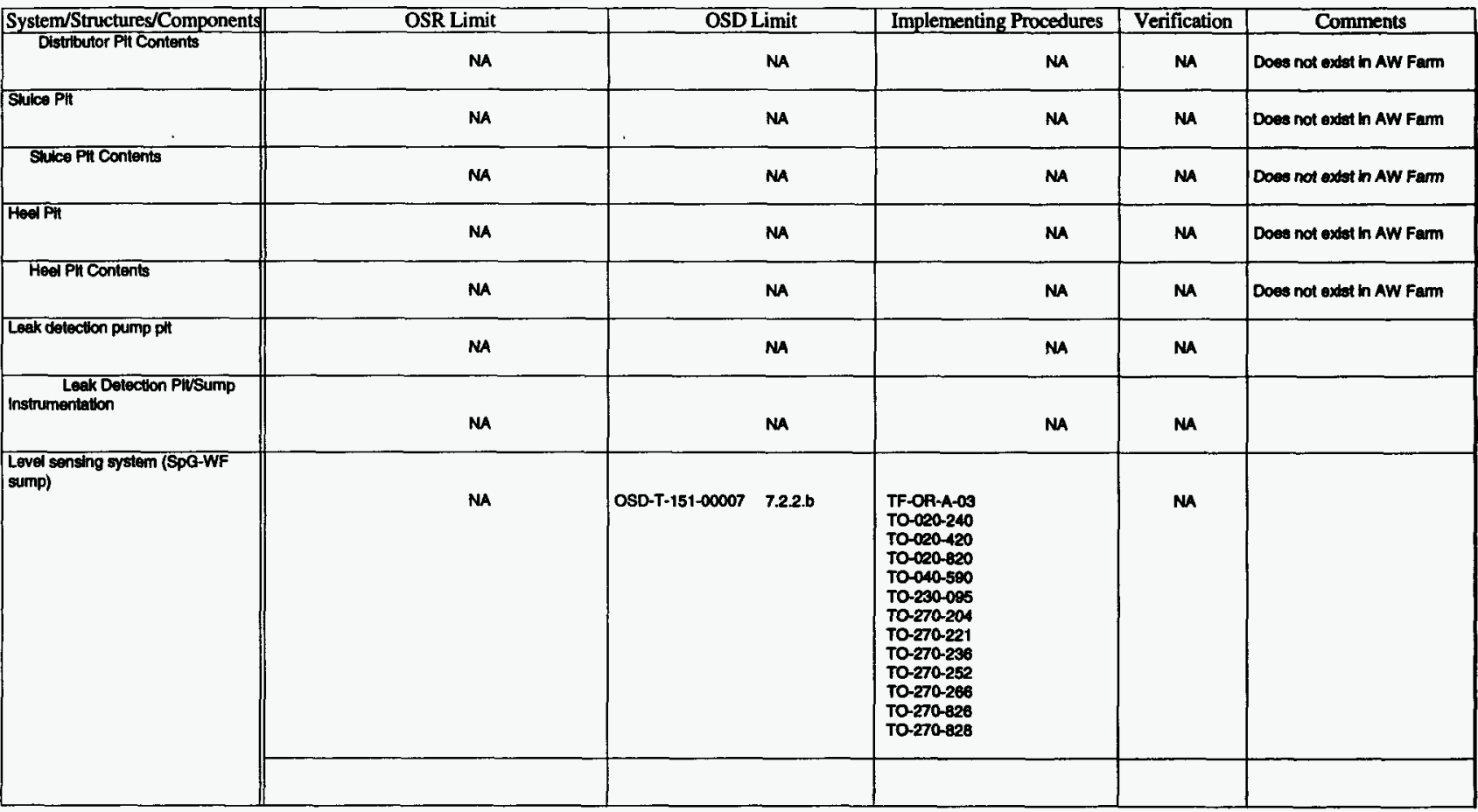

\section{$05 / 31 / 96$}

NA = Not applicable or not required 
TABLE 6

Tank Farm or Miscellaneous Facility

AW

OSR/OSD SAFETY CONTROLS

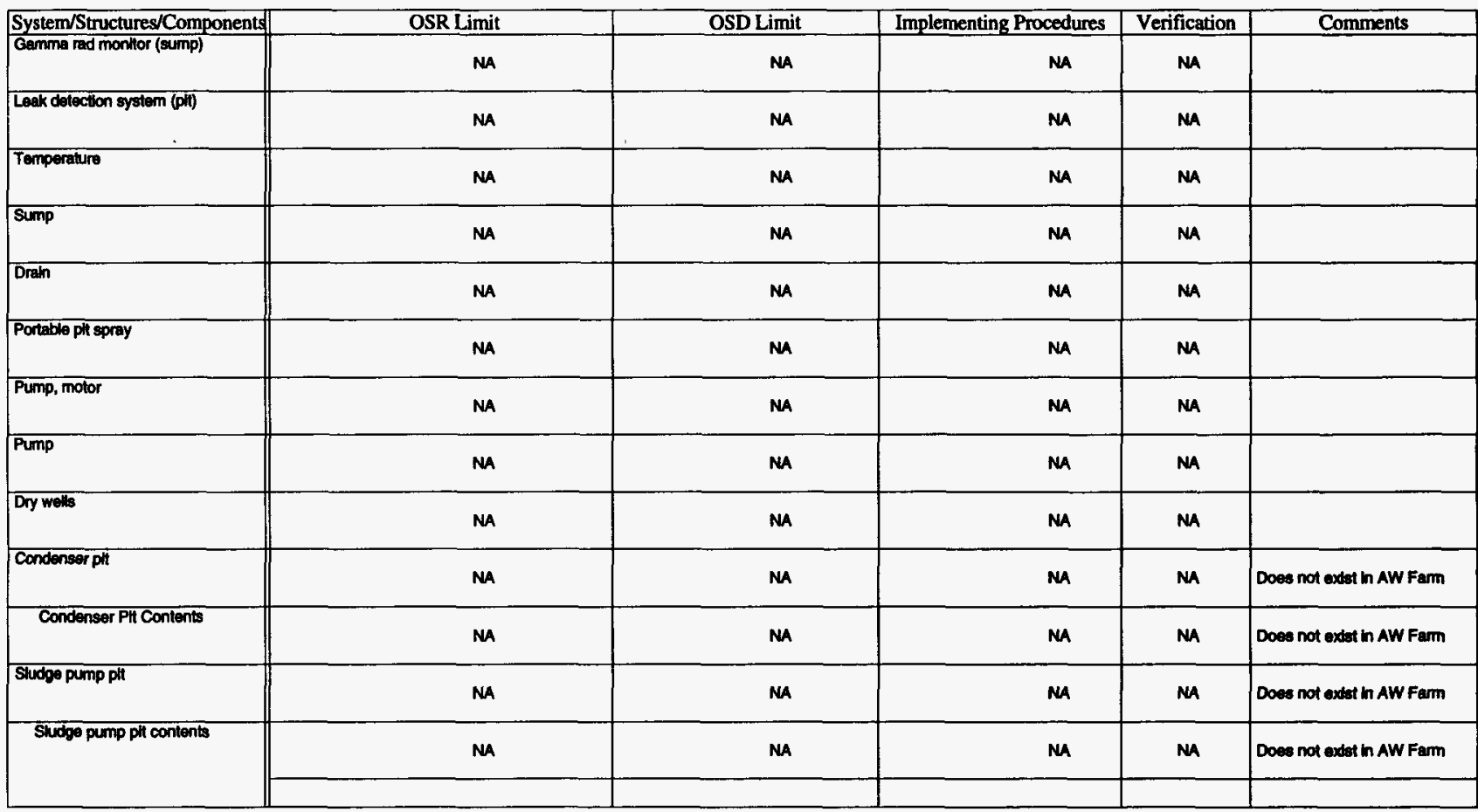

05/31/96

$\mathrm{NA}=$ Not applicable or not required 
Tank Farm or Miscellaneous Facility

TABLE 6

OSR/OSD SAFETY CONTROLS

\begin{tabular}{|c|c|c|c|c|c|}
\hline System/Structures/Components & OSR Limit & OSD Limit & Implementing Procedures & Verification & Comments \\
\hline Selt well pump pit & NA & Na & M & NA & Doea not exdat in AW Farm \\
\hline $\begin{array}{l}\text { Saliwell Pumping } \\
\text { Instrumentation }\end{array}$ & NA & NA & NA & NA & Does not exdst in AW Farm \\
\hline Hydrogen manillor & NA & NA & NA & NA & AW-106 stack \\
\hline Weight factor / specific gravity & NA & NA & NA & NA & AW-102 \\
\hline Drain plt & NA & NA & NA & NA & \\
\hline Drain Ph Contents & NA & MA & NA & NA & \\
\hline Draln & NA & NA & NA & NA & \\
\hline Leak dotection & WHC-SD-WM-SAR-016 $\quad 11.7$ & OSD-T-151-00007 7.2 .10 & $\begin{array}{l}\text { TO-001-184 } \\
\text { TO-220-095 } \\
\text { TO-230-099 } \\
\text { TO-230-133 } \\
\text { TO-230-274 } \\
\text { TO-270-252 } \\
\text { TO-400-200 } \\
\text { TO-430-480 } \\
\text { TO-450-150 }\end{array}$ & NA & \\
\hline Portable pil sprey & NA & NA & NA & NA & \\
\hline 2-10" dia. lines w/ leak detection & NA & NA & NA & NA & Belong to 242-A \\
\hline
\end{tabular}

05/31/96

NA $=$ Not applicable or not required 